\title{
DEVELOPMENT OF AN AMENABLE SYSTEM FOR SITE-SPECIFIC ADDITION TO A MAIZE CHROMOSOME
}

A Dissertation
presented to
the Faculty of the Graduate School
at the University of Missouri-Columbia
In Partial Fulfillment
of the Requirements for the Degree
Doctor of Philosophy
br. James A. Birchler, Dissertation Supervisor
NATHAN CHARLES SWYERS

December, 2019 
The undersigned, appointed by the dean of the Graduate School,

have examined the dissertation entitled

DEVELOPMENT OF AN AMENABLE SYSTEM FOR SITE-SPECIFIC ADDITION

TO A MAIZE CHROMOSOME

Presented by Nathan Charles Swyers,

A candidate for the degree of

Doctor of Philosophy,

And hereby certify that, in their opinion, it is worthy of acceptance.

Dr. James A. Birchler

Dr. Kathleen Newton

Dr. David Braun

Dr. Sherry Flint-Garcia 


\section{ACKNOWLEDGMENTS}

The following list of people helped make this work possible:

\section{Birchler Lab:}

- Patrice Albert

- James A. Birchler

- Weihong Chen

- Robert Gaeta

- Rick Masonbrink

- Ryan Donohue

- Ryan Douglas

- Lin Sun

- Adam Johnson

- Zhi Gao

- Nathaniel Graham

- Becca Lukasak

- Morgan McCaw

- Xiaowen Shi

- Jon Cody

- Hua Yang

- Changzeng Zhao

- Adam Gray

- Alana Rodney

\section{MU Biological Sciences:}

- Rebecca Ballew

- David Braun

- Lori Eggert

- Nila Emerich

- Benjamin Julius

- Melody Kroll

- Kathleen Newton

- Will Swatson

\section{Family}

- $\quad$ Amie Swyers

- Gideon Swyers

- $\quad$ Michael Swyers

- $\quad$ Brenda Swyers

- Charles Swyers

- Rita Henderson

- Michael Noble

- Jill Noble

- $\quad$ Robert Fritz

\section{Friends}

- $\quad$ Seth Nelson

- $\quad$ Leesa Nelson

- John Bauer 


\section{TABLE OF CONTENTS}

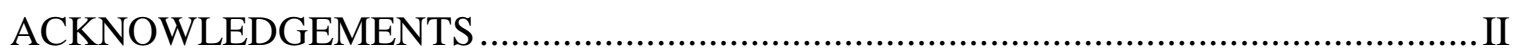

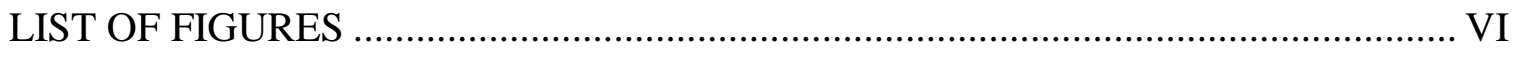

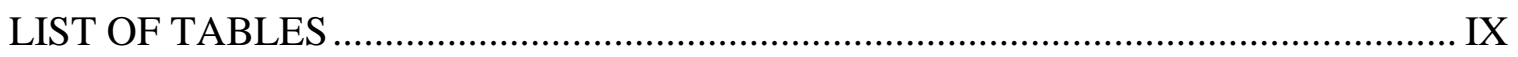

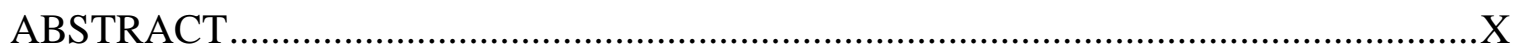

\section{CHAPTER 1:}

1. DEVELOPMENT OF AN AMENDABLE SYSTEM FOR SITE-SPECIFIC ADDTION TO A MAIZE CHROMOSOME........................................................1

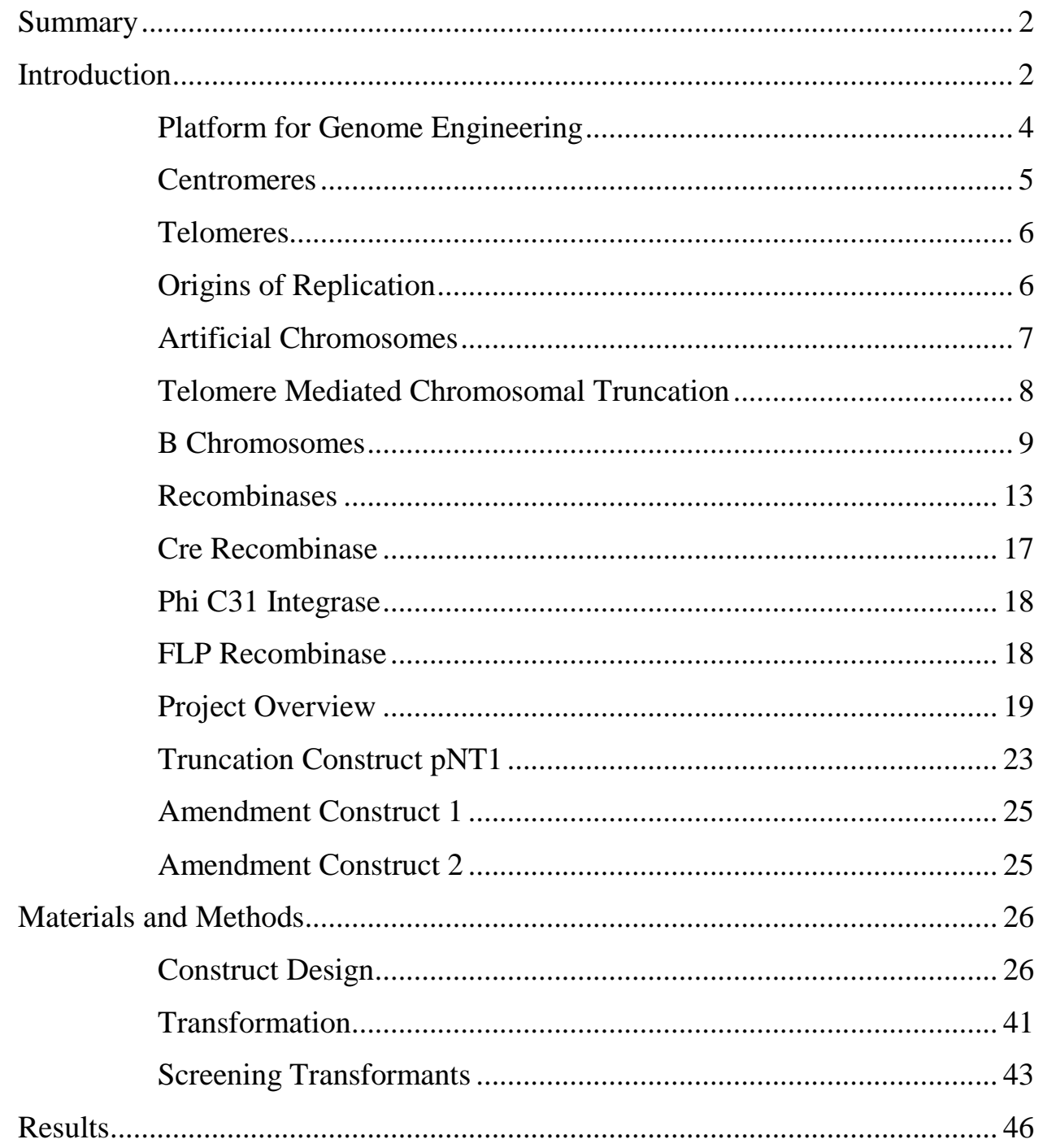


pPZPGLP1 Alternative.... 62

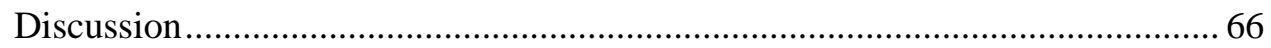

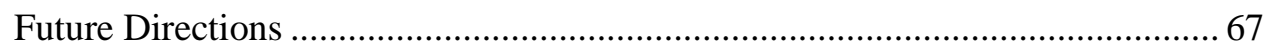

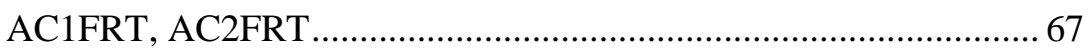

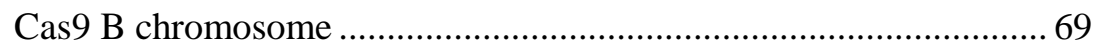

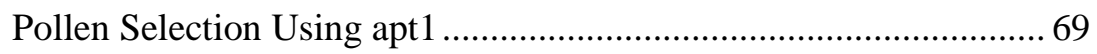

Baby boom and Wuschel Amendment Construct .............................. 70

AC1Telo.............................................................................. 73

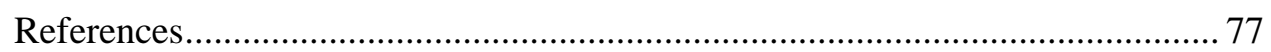




\section{APPENDIX:}

1. AMENDMENT CONSTRUCT MAPS .86

2. TELOMERE-MEDIATED CHROMOSOMAL TRUNCATION FOR GENERATING ENGINEERED MINICHROMOSOMES IN MAIZE ...........101

3. PREPARATION OF CHROMOSOMES FROM ZEA MAYS................................134

4. FLUORESCENT IN SITU HYBRIDIZATION OF MAIZE (ZEA MAYS) CHROMOSOMES 156

5. SEQUENCES OF PNT1, PPZPGLP1, AC1E, AND AC2E. .200 VITA: 


\section{LIST OF FIGURES}

\section{CHAPTER 1:}

FIGURE 1: B CHROMOSOME IN HI II BACKGROUND .................................11

FIGURE 2: RECOMBINASE EXPRESSION CASSETTES.................................16

FIGURE 3: RECOMBINASE RECOMBINATION PRODUCTS .........................19

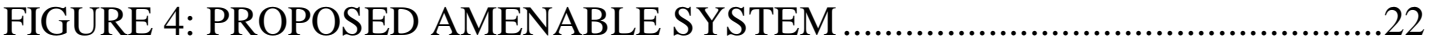

FIGURE 5: PNT1 AND PZPGLP1 CONSTRUCT MAPS …...............................24

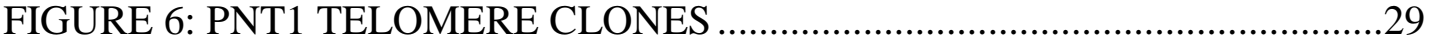

FIGURE 7: SEQUENCING OF PNT1 CONSTRUCT …......................................30

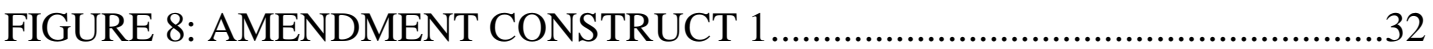

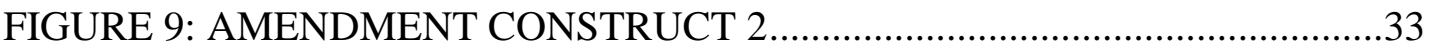

FIGURE 10: AMENDMENT CONSTRUCTS WITH FRT SITES .........................38

FIGURE 11: MAIZE TRANSFORMATION METHODS...................................40

FIGURE 12: PNT1 INSERTED INTO B CHROMOSOME .................................47

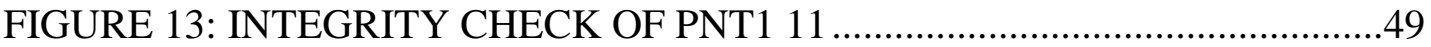

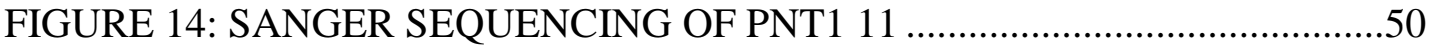

FIGURE 15: CRE-RECOMBINASE SELECTION REMOVAL ............................52

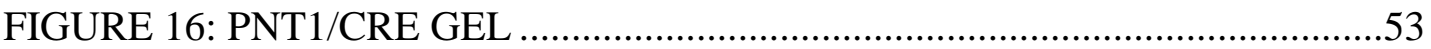

FIGURE 17: FISH IMAGE NEGATIVE CONTROL OF PNT1/CRE ....................55

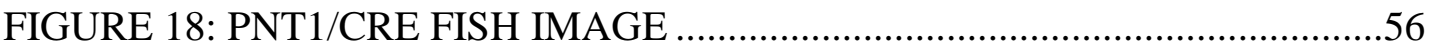

FIGURE 19: SEQUENCING OF CRE-REDUCED PNT1 …................................58

FIGURE 20: SEQUENCING OF CRE-REDUCED PZPGLP1 ............................59

FIGURE 21: PZPGLP1 TARGETING EXPERIMENT COMPONENTS ...............61

FIGURE 22: PZPGLP1 ATTBDSRED AMENDMENT ....................................64

FIGURE 23: PZPGLP1 AC1DSRED AMENDMENT ….......................................65 
FIGURE 24: STRATEGY FOR USE OF BBM AND WUS2

FIGURE 25: AC1TELOMERE POSSIBILITIES .74

\section{SUPPLEMENTAL FIGURES}

SUPPLEMENTAL FIGURE 1: ATTBDSRED EXPERIMENT IMAGE .76

\section{APPENDIX 1:}

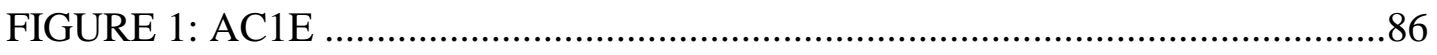

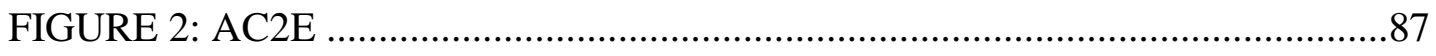

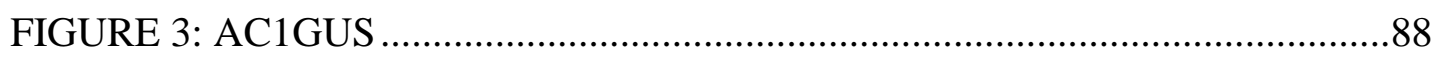

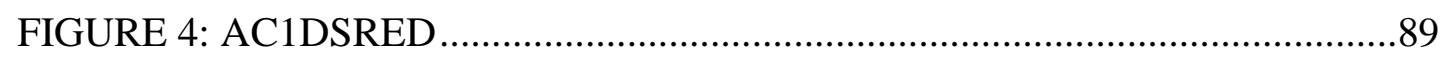

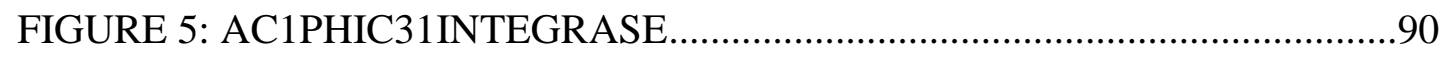

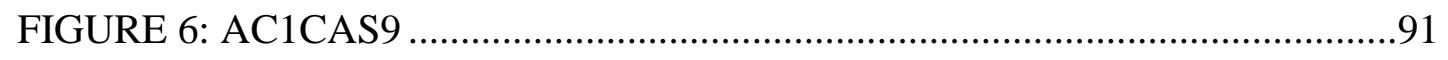

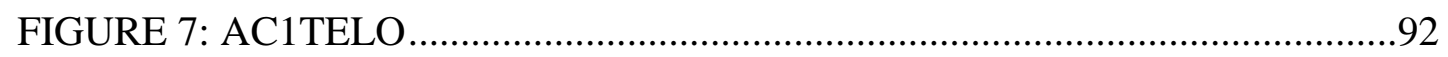

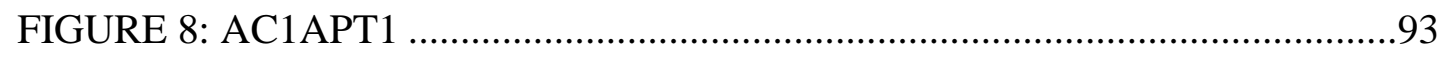

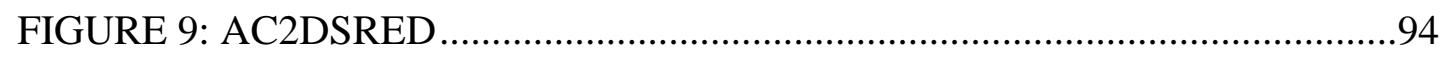

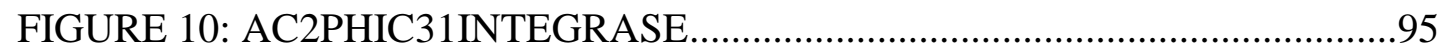

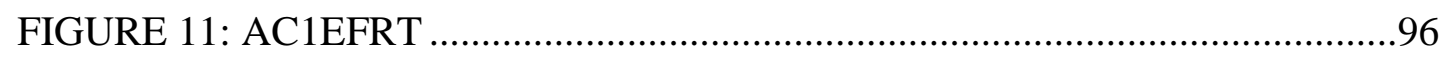

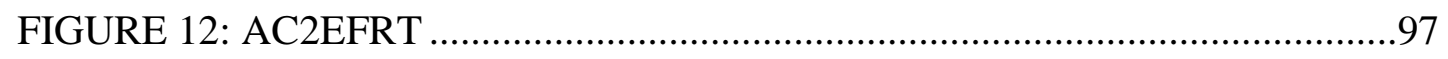

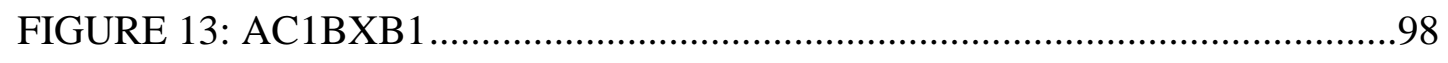

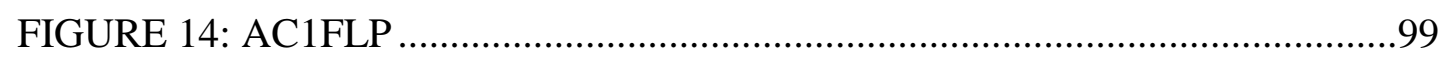

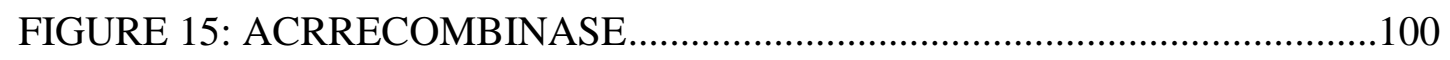

\section{APPENDIX 2:}

FIGURE 1: WY82 DIGEST. 108 


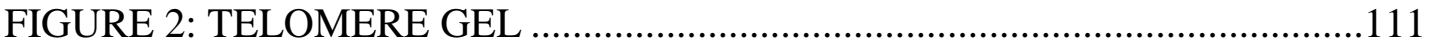

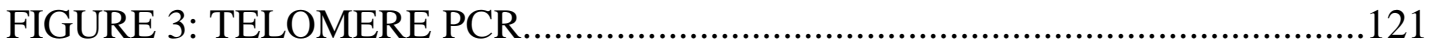

\section{APPENDIX 3:}

FIGURE 1: METAPHASE CHROMOSOME SPREAD

\section{APPENDIX 4:}

FIGURE 1: COMMON SIGNALS TO IDENTIFY CHROMOSOMES

FIGURE 2: BASIC GREEN KARYOTYPE

FIGURE 3: KARYOTYPE OF FFM-B 


\section{LIST OF TABLES}

\section{CHAPTER 1:}

TABLE 1: RECOMBINASE BINDING SEQUENCES .12

TABLE 2: PRIMERS USED FOR SCREENING .45

\section{APPENDIX 4:}

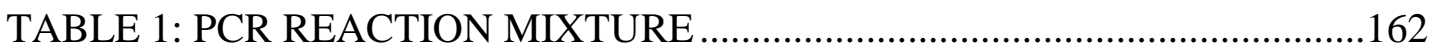

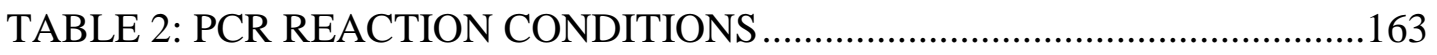

TABLE 3: PLASMIDS FOR AMPLIFYING MAIZE PROBES ...........................175

TABLE 4: PRIMER SEQUENCES FOR AMPLIFYING MAIZE PROBES .........176

TABLE 5: PROPES USED FOR MAIZE KARYOTYPE ..................................177

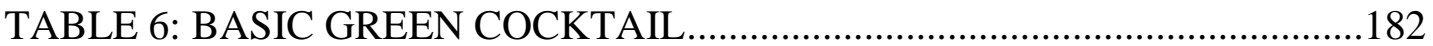




\section{Abstract}

Currently, transgenic maize is produced by random integration of a transgenes into the plant. This works for single genes, but not as well for multiple traits. Identifying plants that contain several transgenes becomes a very difficult task. Gene stacking at a single location in the genome would make combining multiple transgenes into plants a simpler process. This project focused on the development of a system would allow for transgenes to be sequentially added to a specific site in the maize genome. The system utilizes two recombinases, Cre recombinase and $\phi C 31$ Integrase, to remove a selectable marker and to integrate transgenes. An initial construct containing a selectable marker, flanked by LoxP sites, which are acted upon by Cre recombinase, and an attP site, were transformed. The selectable marker was then removed from the integrated transgene by exposure to Cre recombinase. Two amendment constructs enable modification of the integrated construct by utilizing complementary attP and attB sites, which are acted upon by $\phi C 31$ Integrase. The amendment constructs contain cargo and a promoterless selectable marker which, upon successful recombination with the target site, restores expression of the selectable marker. Successful demonstration of this system simplifies generation of multi-transgene plants, and the assembly of multi-gene pathways in plants. 


\section{Chapter 1: Development of an Amenable System for Site-Specific Addition to a Maize Chromosome}

Note: The information provided in this chapter is unpublished.

Contributions

Swyers N.C., Jon P. Cody ${ }^{1}$, Nathaniel D. Graham ${ }^{1}$, Chanzeng Zhao ${ }^{1}$, and James A. Birchler ${ }^{*}$

\section{Author Contributions}

NS established the pNT1 transgenic lines, created all Amendment Constructs, created pIntFLPremBAR and its transgenic lines, and performed all bombardments of pNT1 and pPZPGLP1 with AC1DsRed and attBDsRed shown in this chapter. NS performed Cre-mediated excision of BAR from pNT1. NS performed all screens on PNT1 and pPZPGLP1 material to be used in experiments in this chapter. JC created the attBDsRed, and plntHyg constructs as well as provided invaluable guidance and feedback. NG created pPZPGLP1 and its transgenic lines, performed Cre-mediated excision on PPZPGLP1 lines, and crossed some of these to phiC31 Integrase. NG performed initial experiments using GUS expression to show functionality of phiC31 Integrase, as well as provided valuable guidance. $\mathbf{C Z}$ provided guidance and feedback on 
performing maize transformation as well as created phiC31 Integrase expression lines.

\section{Summary}

Site-specific recombinase enzymes function in heterologous cellular environments to initiate strand-switching reactions between unique DNA sequences termed recombinase binding sites. Integrations, excisions, or inversions of specified DNA sequences result predictably from recombinase reactions depending on the position and orientation of recombinase binding sites. Here, we demonstrate the ability to modify a transgene in the maize genome by both excision and site-specific integration. Cre-recombinase was used to excise a resistance marker from a transgene integrated into the maize genome, followed by site-specific integration of two plasmids separately to the same transgene by phiC31 Integrase. The necessary components were constructed to allow for repeated site-specific integrations into a single transgene location and, since all individual components have been shown to work, future directions to implement fully this plan are provided.

\section{Introduction}

The need to increase agricultural productivity is paramount to address a rapidly growing world population, and rising global temperatures. The amount of arable land on the surface of the earth is finite, and because of the growth of human populations and climate change, the amount of arable land per capita is decreasing (Benke, and Tomkins, 2017). In the past, increases in crop productivity were made by traditional breeding. This process, over thousands of 
years, has resulted in many of the crops we have today. Traditional breeding, however, is limited by the natural variation of each crop species and takes many generations for isolation of only beneficial traits (Hirschi, 2009; Zhu et al. 2007). The ability to integrate a foreign gene fragment into the genome of a plant (Bevan et al. 1983; Hererra-Estrella et al. 1983; Fraley et al. 1983; Murai et al. 1983) opened a new avenue for crop improvement through genome engineering. The field of genome engineering has grown rapidly over the last decade as new tools and technologies have enabled researchers, in a greater capacity than ever, the ability to change the genome of an organism as the researcher sees fit. With the advent of such technologies, prospects for major improvements to our understanding of the underlying genes that give rise to traits should enable researchers to improve the quality and nutrition of our crops. The convergence of recombinase technology, genome sequencing, and precise gene editing tools will allow researchers to identify target genes and precisely edit them, or even clone these genes into a specific site in a different species. For example, many important vitamin pathways require that multiple genes be expressed. Through genome sequencing, researchers can identify the genes that create a specific vitamin. Cloning and recombinase technologies can then be used to take those genes and stack them into a specific location in a different species, recapitulating a specific pathway that may have not been present, or complete, in the target species. Random integration of several transgenes makes selection for plants that contain all the transgenes difficult because of segregation and independent assortment during meiosis. Stacking genes in one position in the genome would 
make selection of plants containing the genes of interest much easier than if one must follow genes of interest that are randomly distributed throughout the genome.

\section{Platform for Genome Engineering}

Random integration of transgenes not only makes selecting for multiple transgenic traits difficult but also has several other disadvantages. The genomic location and environment of transgene insertion can have dramatic effects on the expression of the transgene (Matzke and Matzke 1998; Vaucheret et al. 1998). Transgenes can be inserted into genes necessary for the plant's survival, resulting in death. Random integration of transgenes into linkage blocks can result in the linkage of negative alleles with the transgene when crossing into commercial lines. Also, transgenes may be inserted into regions of the genome that are heavily methylated, resulting in the silencing of the transgene. Some of these disadvantages can be overcome by the development of a platform for transgene stacking. The ideal platform would show faithful transmission from one generation to another, allow stable expression, result in no linkage drag, and would not result in unwanted detrimental effects toward the host. An artificial chromosome that exists outside of the normal genome would be an ideal target for genome engineering. Creating an artificial chromosome would require the formation of, at minimum, three basic components: centromeres, telomeres, and origins of replication. These pieces would enable the artificial chromosomes faithful transfer from generation to generation. 


\section{Centromeres}

Centromeres are regions of the chromosome that are required for faithful separation of sister chromatids to either pole of a cell during anaphase. They are the sites of kinetochore formation and where spindle fibers bind to the chromosomes (Cody et al. 2015). While centromeres are determined by specific DNA sequences in yeast, centromere determination in most eukaryotes is more complex. The centromeres of plants are generally composed of satellite repeats that can vary in number between species and sometimes chromosomes (Kanizay et al. 2009; Burrack et al. 2012; Kato et al. 2004). Centromere activity in plants is determined by localization of a histone $\mathrm{H} 3$ variant (CENH3) (Comai et al., 2017). CENH3 interacts with satellite repeats and retrotransposons, which are interspersed in centromeric regions (Zhong et al. 2002). Studies have also shown that de novo centromeres can be formed over unique DNA sequences, whereas most genomic centromeres share similar sequence composition (Fu et al. 2013). Centromeres can also be inactivated as was shown in maize by the recovery of a dicentric B chromosome (Han et al. 2006). The discovery of both de novo activation and inactivation of plant centromeres shows that centromere formation has an epigenetic component, rather than determination by DNA sequence alone. The epigenetic nature of plant centromeres means that simply including centromeric repeats into an artificial chromosome is unlikely to result in kinetochore formation and centromeric function. 


\section{Telomeres}

Telomeres are composed of repetitive sequences and a protein complex that serves as the caps for the ends of chromosomes. These caps protect chromosomes from end-to-end fusion and damage to the DNA during replication. Telomere regions are highly heterochromatic, although the ends of telomeres consist of a single strand overhang of DNA that forms what is known as a GLoop (Makarov et al. 2001). Telomeres, in contrast to centromeres, are designated by a specific DNA sequence, which is highly conserved across many plant species. In most plants, including maize, TTTAGGG serves as the telomeric repeat. DNA polymerase requires a primer template for extension, which results in DNA at the ends of chromosomes being lost after each round of DNA replication, causing telomere shortening. Telomere repeat arrays can be recognized and extended by telomerase. Telomerase works as a reversetranscriptase, using the telomere's $3^{\prime} \mathrm{OH}$ as a primer, extending out the telomere repeat as necessary. Telomerase activity, however, varies by tissue and shows little activity in mature tissues (Fitzgerald et al. 1996; Kilian et al. 1998). For the creation of an artificial chromosome, telomere sequences can be introduced into a chromosome and create a new end to the chromosome at the transgene insertion site in a process known as telomere-mediated chromosomal truncation (Yu et al. 2006).

\section{Origins of Replication}

Origins of replication serve as locations for DNA synthesis during the replication phase of the cell cycle. Replication of the genome and DNA synthesis are 
extremely important steps in the reproduction of the cell and are thus tightly regulated. In many prokaryotes there exists only one origin of replication, which dictates that it must be active for synthesis to occur. In eukaryotic chromosomes, there are many origins of replication that are active at different times during replication (Leonard et al. 2013). In metazoans these replication origins are not

all active in all cells (Leonard et al. 2013). S. cerevisiae is the only eukaryote for which the origin of replication has been identified, and neither a consensus sequence nor specific location has been found in other eukaryotes (Leonard et al. 2013). Without knowledge of the constitution of an active origin of replication in the target species, synthesized origins of replication cannot be created.

\section{Artificial Chromosomes}

Artificial chromosomes can be developed from either a "top-down" or "bottom-up" approach (Gaeta et al. 2012). The bottom-up approach relies on the ability to aggregate a centromere, telomeres, and origin of replication (Gaeta et al. 2012). This has been accomplished in yeast (Murray and Szostak 1983) and in human cells (Henning et al. 1999), but, because of the epigenetic nature of centromeres and unknown origins of replication in other eukaryotes, has not been accomplished in most species. A second option for creating an artificial chromosome is by utilizing a so-called top-down approach. The top-down approach is accomplished by utilizing the machinery of an existing chromosome to create the artificial chromosome (Gaeta et al. 2012). One method that has been successfully demonstrated in several plant species, including maize, is telomere mediated chromosomal truncation (Yu et al. 2006). 


\section{Telomere Mediated Chromosomal Truncation}

Telomeres form the natural ends of chromosomes, and consist of arrays of repetitive DNA sequence. These repeats are both necessary and sufficient for the organization of the DNA capping machinery. Telomere-mediated chromosomal truncation utilizes the innate ability of telomere repeat arrays to attract telomerase and the chromosome capping machinery to form a new end to a chromosome. Telomere-mediated chromosomal truncation has been demonstrated in several species including, maize (Yu et al. 2006), Arabidopsis (Nelson et al. 2011; Teo et al., 2011), wheat (Yuan et al. 2017), barley (Kapusi et al., 2012), rice (Xu et al., 2012), and Brassica (Yan et al. 2017). To accomplish telomere-mediated truncation of a chromosome, synthetic telomere repeat arrays are added to a construct. When the construct is inserted into a double-strand break in a chromosome, the telomere arrays on the transgene may be recognized as the new end of the chromosome. Generally, the introduced telomere sequence is shorter than the endogenous telomere sequence that it is replacing. The introduced telomere sequence must therefore be extended and be modified to have a G loop structure (Makarov et al. 2001). The result of this truncation is a shortened chromosome with the telomere-containing transgene at the terminal end, and an acentric fragment from the distal end of the chromosome, which is lost. The resulting truncated chromosome is referred to as a minichromosome. The loss of the distal tip of a chromosome can be detrimental, even lethal, to the organism. To avoid lethality, a number of strategies have been developed including utilization of: telo-trisomics (Xu et al. 
2012), tetraploids (Yu et al. 2007; Teo et al. 2011; Kapusi et al. 2012), and B chromosomes (Yu et al. 2007). In maize, the B chromosome has been identified as a particularly attractive target for truncation (Gaeta et al. 2012).

\section{B Chromosomes}

The ideal target for genome engineering lacks essential genes, avoids linkage drag, and can be followed faithfully generation to generation. B chromosomes exist outside the normal chromosome complement and often deviate from Mendelian segregation, displaying preferential inheritance (Houben 2017). These chromosomes exist in several species across eukaryotes, including maize. In both maize and rye, B chromosomes display post-meiotic drive. The B chromosome of maize has no known function or vital genes (Carlson 1978). It displays the property of nondisjunction at the second pollen mitosis (Roman 1947). This nondisjunction is linked to a region on the distal tip of the long arm of the B chromosome. The property of nondisjunction allows the B chromosome to increase in number in the plant. Nondisjunction is lost if the distal tip of the $B$ chromosome is lost. Sperm containing B chromosomes also preferentially fertilize the egg, a property that helps maintain the B chromosome in populations. Figure 1 displays some unique features of the $\mathrm{B}$ chromosome shown by Fluoresence In Situ Hybridization (FISH). The B chromosome has a very small short arm and a long arm of a size similar to the smaller A chromosomes such as chromosomes 9 and 10. The B chromosome can also be identified by the unique binding locations of certain FISH probes. The TAG repeat probe binds strongly to the middle of the long arm of the B chromosome. The CentC repeat probe binds 
weakly along various sections the B chromosome and also weakly to the centromere. TAG and CentC probes, in green, are shown on Figure 1. 


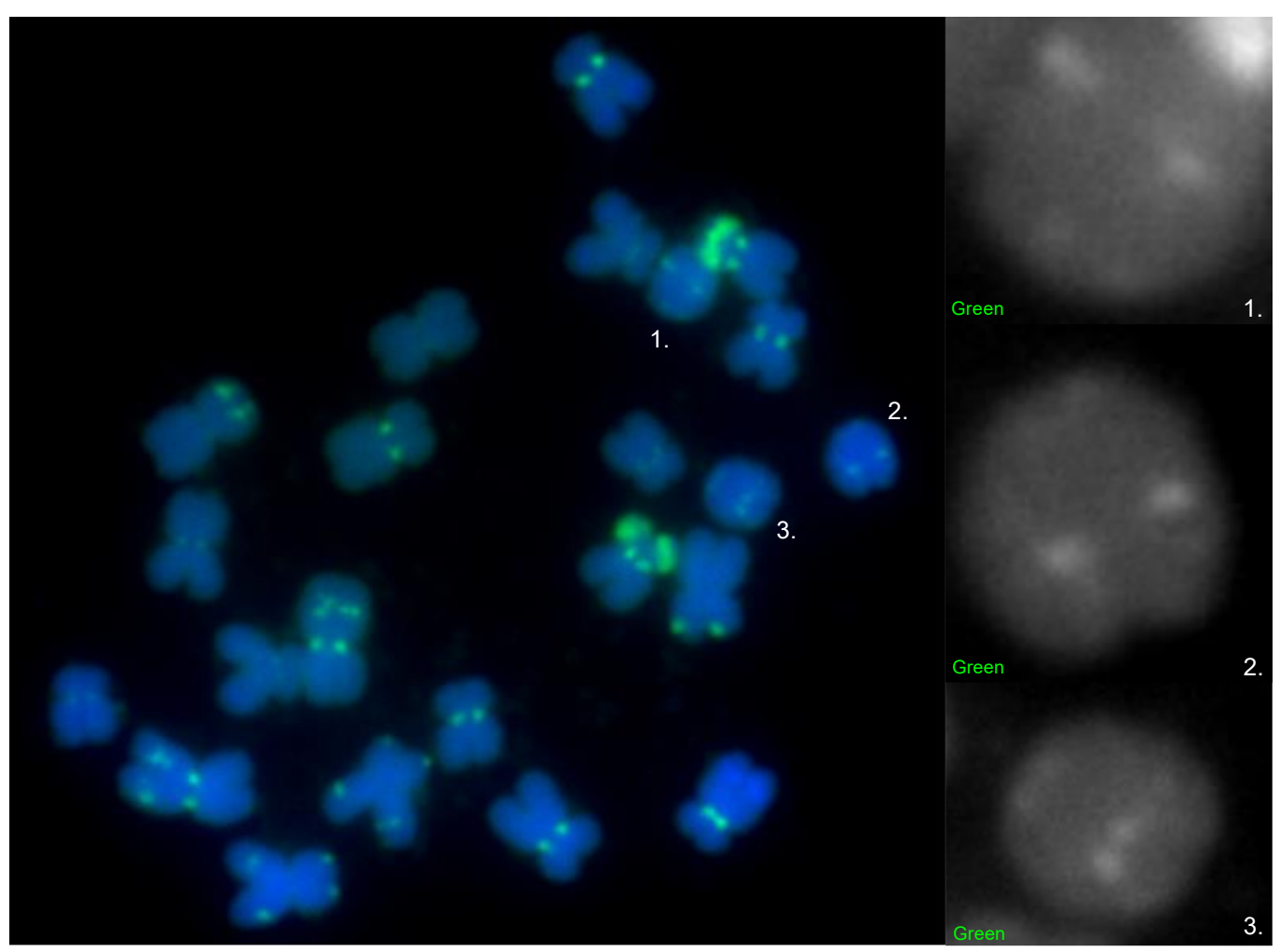

Figure 1: B Chromosomes in Hi II Background

FISH image of a Hi II plant containing three $\mathrm{B}$ chromosomes. Chromatin is stained blue by DAPI. In green, labeled with AlexFluor, is TAG and CentC repeats as well as the nucleolar organizing region (NOR). TAG highlights different regions on different chromosomes which helps in karyotyping a given nucleus. Importantly TAG displays a strong signal in the middle of the long arm of the $B$ chromosome. CentC is a centromeric repeat that varies in strength depending on the chromosome, which also helps in identifying individual chromosomes. CentC also binds to many parts of the B chromosome which can give it a "speckled" appearance. 


\begin{tabular}{|c|c|c|}
\hline Recombinase & Binding Sequence & Directionality \\
\hline Ore - Iox & 5' ATAACTTCGTATAATGTATGCTATACGAAGTTAT 3' & Bidirectional \\
\hline PhiC31 Integrase & $\begin{array}{l}\text { attB - 5' GTGCCAGGGCGTGCOCTTGGGCTCCOCGGGCGCG 3' } \\
\text { attP- 5' COCCAACTGGGGTAACCTTGAGTCTCTCAGTTGGGGG 3' }\end{array}$ & Unidirectional \\
\hline FPe - FRT & 5' GAAGTTCCTATTCTCTAGAAAGTATAGGAACTTC 3' & Bidirectional \\
\hline
\end{tabular}

Table 1: Recombinase Binding Sequences

Recombinases and their binding sequences. Directionality of the recombination reaction is also provided. 


\section{Recombinases}

In eukaryotic chromosomes, integration of introduced DNA into the genome occurs naturally, though the process of this integration is both random and infrequent (Keravala et al. 2008). Transposases and retroviral integrases can be used to increase the frequency of integration, but the resulting integrations are nearly random, and these enzymes have strict size limits on the amount of DNA to be integrated (Keravala et al. 2008). Site-specific recombinases offer solutions to the integration capacity issue, as well as the issue of random integration, while also keeping the benefit of a higher integration efficiency. Cre recombinase was the first of these enzymes to be used widely for genome modification (Dale and Ow 1990), but many others have been used since including: FLP recombinase (Lyznik et al. 1993), phiC31 Integrase (Thompson et al. 2010), R recombinase (Nakagawa et al. 2001), CinHRS2 (Moon et al. 2011), and Bxb1 Integrase (Li et al. 2016). Cre and FLP both work by recognition of and recombination within teo identical 34 bp sites. Although these enzymes can be used for integrations, in many cases these integrations are quickly reversed by deletion. This action makes Cre and FLP enzymes more useful for making precise deletions (Keravala et al. 2008). Integrases, such as phiC31 and Bxb1, recombine between two nonidentical sequences, which creates hybrid sites that cannot undergo further recombination by their respective enzymes without a secondary molecule. The non-reversibility of the integrases makes them ideal for integration into chromosomes. In order to create a system for sequential site-specific addition to the maize genome, a strategy was devised based on previous work (Ow 2011). 
For this system to function, there must be a rotation between the actions of two separate recombinases. Our strategy relies on the use of Cre recombinase and phiC31 Integrase, which belong to separate recombinase families, which are the resolvase/invertase integrase and the lambda integrases, respectively. These two major classes of recombinases utilize a different amino acid residue to catalyze recombination: Tyrosine and Serine. A third recombinase, FLP recombinase, has a potentially important place in the system and will be described. Expression of these three recombinases is provided by the constructs in Figure 2 and recombinase binding sites are provided in Table 1. 


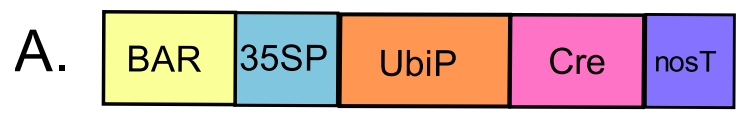

\begin{tabular}{|l|l|l|l|l|}
\hline HygR & $35 \mathrm{SP}$ & UbiP & phiC31 Integrase & nosT \\
\hline
\end{tabular}

\begin{tabular}{|c|c|c|c|c|c|c|c|c|}
\hline $35 \mathrm{SP}$ & BAR & nosT & nosT & phiC31 Integrase & nosP & nosP & Flippase & nost \\
\hline
\end{tabular}

\begin{tabular}{|l|l|l|l|l|}
\hline BAR & $35 \mathrm{SP}$ & UbiP & phiC31 Integrase & nosT \\
\hline
\end{tabular}

\section{Figure 2: Recombinase Expression Cassettes}

A: Cre-expression cassette to be used for removal of BAR-nosT from pNT1. This transgene was previously transformed into the maize genome. Crossing pNT1 containing plants to plants expressing Cre recombinase from this transgene will result in the removal of BAR-nosT from pNT1.

B: phiC31 Integrase expression cassette to be used in tandem with amendment constructs to facilitate their site-specific integration into pNT1. This cassette is cobombarded with amendment constructs.

C: FLPase and phiC31 Interase expression cassette. This cassette is designed for the expression of both phiC31 Integrase and FLPase to allow for circularizaton and integration of amendment constructs when they are transformed using Agrobacterium transformation. BARnosT is flanked by lox71/66 sites which will allow for their removal when exposed to Cre recombinase.

D: phiC31 Integrase expression cassette, which contains a BAR selectable marker. This construct has been crossed to plants containing pPZPGLP1 (Figure 5). This allows for testing if site-specific integration via the amenable system is possible but will not allow for regeneration of positive transformants. 


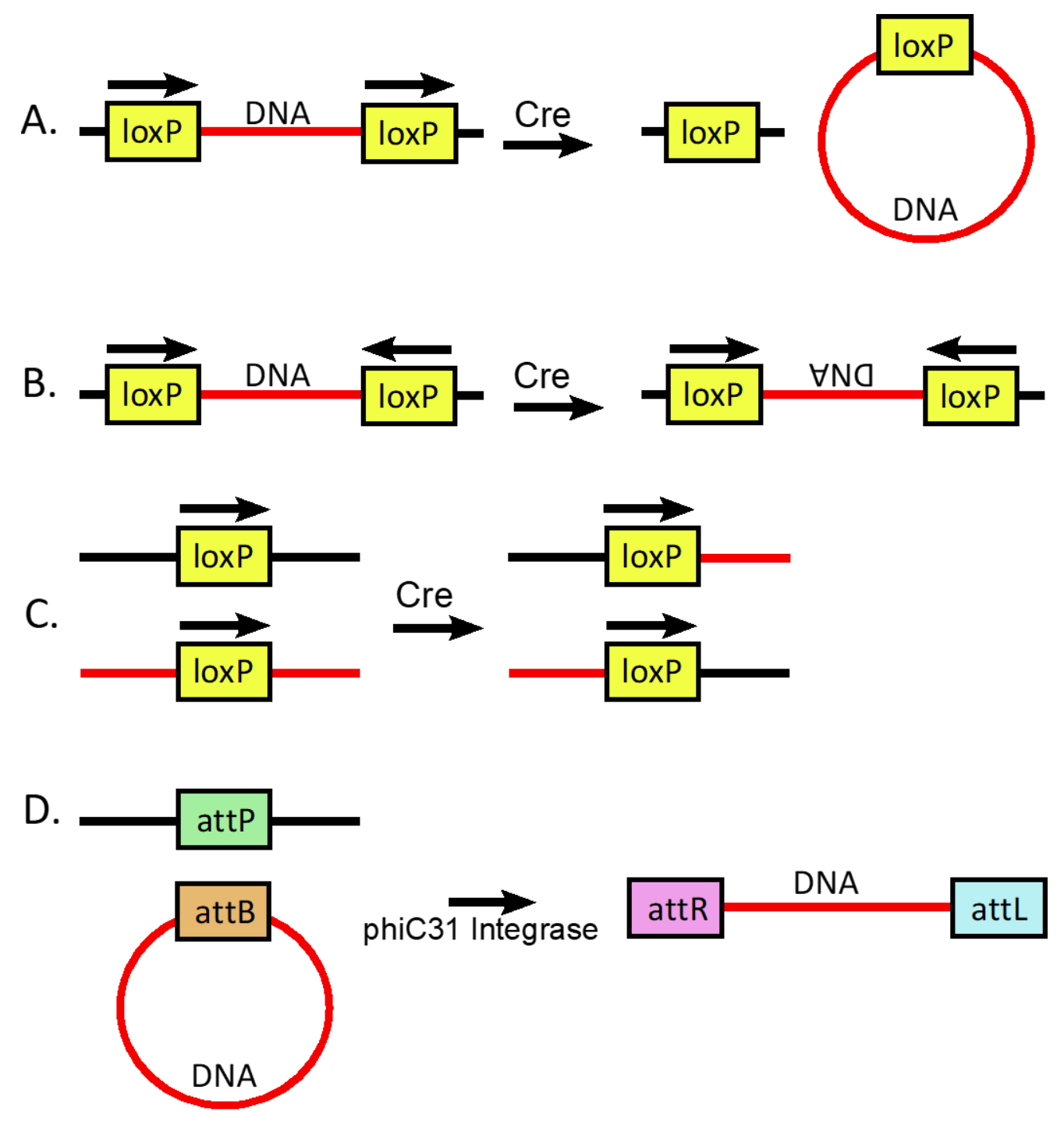

Figure 3: Recombinase Site Orientation and Its Effect on Recombination Products

A. Exposure of recombination sites, in this case LoxP, to their respective recombinase, in this case Cre, with sites oriented facing the same direction, results in intervening DNA being removed.

B. Recombination sites oriented inwards towards intervening DNA results in the flipping of that DNA when exposed to the recombinase.

C. Recombination between recombinase sites on separate linear DNA molecules results in strand switching between the two.

D. Recombination between one recombinase site (here attP) on a linear DNA molecule and a second complementary site (here attB) on a circular DNA molecule results in the integration of the circular DNA into the recombination site on the linear DNA molecule. 


\section{Cre recombinase}

Cre recombinase is a member of the Tyrosine family of recombinases and is derived from the P1 bacteriophage (Garcia-Otin, A. L., et al. 2006). In the bacteriophage, Cre performs an important role as a resolvase during replication of the phage genome by cutting and rejoining the duplicated phage genome into two similar pieces (Garcia-Otin et al. 2006). The Cre enzyme catalyzes recombination between lox sites. Lox sites are 34 basepairs long and consist of two palindromic $13 \mathrm{bp}$ long arms surrounding an 8 bp non-symmetrical core sequence that determines the orientation of the lox site. In order to perform recombination, Cre enzyme forms a tetrameric structure to stabilize the synaptonemal complex. Opposite Cre subunits (Cre enzyme dimers) cleave and exchange a pair of DNA strands, which forms a Holliday junction. Isomerization of this DNA intermediate causes activation of the second Cre subunits, which catalyzes a second cleavage and strand exchange that completes recombination (Garcia-Otin et al. 2006). A phosphotyrosine linkage is made transiently between the DNA and the enzyme, which catalyzes the reaction. Depending on the orientation of lox sites, Cre-mediated recombination can cause exchange, removal, or flipping of the DNA between lox sites as shown in Figure 3. Lox sites oriented in the same direction will cause intervening DNA to be excised, leaving behind one lox site. Lox sites facing inwards cause intervening DNA to be inverted, when acted upon by Cre recombinase. Importantly, recombination 
between lox sites is bidirectional, as recombination between lox sites result in intact lox sites.

\section{PhiC31 Integrase}

PhiC31 Integrase is derived from the Streptomyces phage. It is a member of the resolvase/invertase family of integrases. This family is characterized by its mode of recombination, which involves making a four-strand break in the DNA and rejoining via a phophoserine linkage that is formed between the enzyme and DNA (Thorpe et al. 1998). PhiC31 integrase acts upon sites known as attP (39 base pairs long), and attB (34 base pairs long) sites. The integrase recombinase binds as a dimer to each attP and attB site. The dimers act as a tetramer when attP and attB sites are near one another. The formation of the tetramer allows cleavage of the attP and attB sites, after which strand exchange occurs between the two cleaved sites. The resulting hybrid att sites are designated attL and attR in Figure 3. The resulting attL and attR sites cannot be cleaved by the integrase enzyme without a secondary enzyme named excisionase. This means that recombination between attB and attP sites is effectively unidirectional (without the presence of excisionase). In a manner similar to how lox sites operate, the arrangement and direction of the attachment sites determines the outcome of recombination between attachment sites.

\section{FLP Recombinase}

FLP recombinase is a member of the lambda integrase family of recombinases. FLP is derived from Saccharomyces cerevisiae and is the eukaryotic equivalent of the bacteriophage P1 Cre system (Yamada et al. 2014). The FLP enzyme acts 
upon 34 bp sites called FRT sites. Like lox sites, FRT sites have directionality based on their core sequence. The orientation of FRT sites, as with the other recombinases described above, determines the results of recombination: excision, reversion, or integration. Recombination between FRT sites requires 4 FLP molecules and 2 FRT sites (Yamada et al. 2014). Like Cre and other lambda integrases, FLP is primarily used for site-specific deletion because using the system for integration often results in the deletion of the introduced DNA.

\section{Project Overview}

The goal of this project was to create a minichromosome via telomere mediated truncation of a B chromosome, and then to utilize the recombinases described above to sequentially modify and add cargo to it. The resulting modified minichromosome would demonstrate the utility and viability of using this system for site-specific gene stacking. Three hurdles must be overcome to complete this demonstration: truncation/insertion of a B chromosome, removal of the selectable marker from the initial insertion site, and site-specific amendment to the insertion. To demonstrate the ability to stack genes in a site-specific manner, a series of constructs must be utilized (Figure 4). The first construct is designed to be a landing pad for further modification using downstream constructs in conjunction with Cre-recombinase and phiC31 Integrase. To accomplish truncation of a chromosome, this construct also includes $2 \mathrm{~kb}$ of telomere DNA. To allow for modification of the insertion by recombinases once inserted into the $B$ chromosome, their appropriate recombination sites are included in the construct. This initial construct must also contain a selectable marker to allow for selection 
of positive transformants. The selectable marker chosen for this experiment was the gene BAR, which confers resistance to bialaphos herbicide. The selectable marker must be removable so that subsequent selection can be restored via sitespecific modification. Lox sites surround the selectable marker and are oriented in the same direction, allowing for removal of the selectable marker when exposed to Cre-recombinase. An attP site is also included so that an Amendment Construct, containing a complementary attB site, can site-specifically modify the initial insertion, bringing cargo and restoring selection. These secondary constructs, referred to as Amendment Constructs 1 and 2, allow for the restoration of the selectable marker by phiC31 Integrase-mediated recombination between the genomic site and the amendment constructs. These amendment constructs also contain cargo. Amendment construct 1 contains an attB site for recombination with the initial construct's attP site. Amendment construct 2 contains an attP site for modification of the modified initial contruct's attB site. Switching between these two amendment constructs should allow for sequential addition of multiple genes into a specific site in the genome. For the system to work, a Cre-recombinase expressing maize line is used to remove the selectable marker between exposures to the amendment constructs. Expression of phiC31 Integrase is also required and can be accomplished either by cotransformation of an Integrase expressing plasmid with an amendment construct or through a stable Integrase expressing maize line. After amendment of the insertion using Amendment Construct 1, the three major goals of the project; truncation/insertion of a B chromosome, removal of Bialaphos (BAR) selectable marker, and site- 
specific amendment to the initial construct would be complete. Theoretically, by alternating between Amendment Constructs 1 and 2 after intervening rounds of Cre-mediated BAR removal, cargo can be added sequentially in a site-specific manner to the initial truncation construct site indefinitely. 
A.

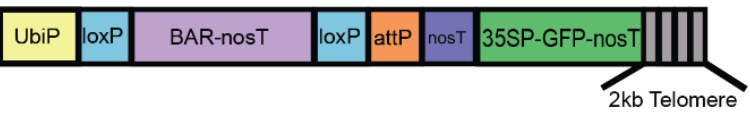

B. + Cre

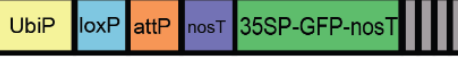

C.

+Integrase +Amendment Construct 1

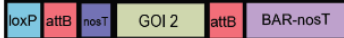

Amendment Construct

\begin{tabular}{|l|l|l|l|l|l|l|l|l|l|l|l|}
\hline UbiP & loxP & attR & BAR-nosT & Backbone & loxP & attB & nosT & GOI 2 & attL & nost & 35SP-GFP-nosT \\
\hline
\end{tabular}

D. + Cre

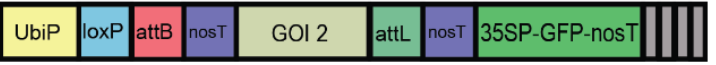

E. +Integrase +Amendment Construct 2

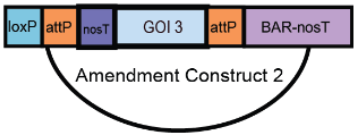

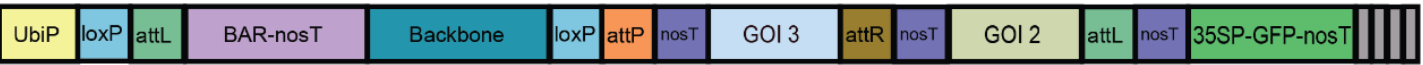

F. + Cre

\begin{tabular}{|l|l|l|l|l|l|l|l|l|l|l|l|}
\hline UbiP & loxp & attP & nost & GOI 3 & attR & nosT & GOI 2 & attL & nost & 35SP-GFP-nosT & | \\
\hline
\end{tabular}

\section{Figure 4: Proposed Amenable System}

A: Integration of the initial construct, pNT1, which includes the selectable marker BAR, removable via Cre recombinase and the surrounding LoxP sites, driven by a ubiquitin promoter, followed by an attP site for further modification using integrase. GFP driven by a $35 \mathrm{~S}$ promoter is included as cargo. $2 \mathrm{~kb}$ of telomere DNA is included to induce truncation. B: Exposure to Cre recombinase results in removal of BAR selection and a LoxP site. C: Expression of phiC31 Integrase in combination with inclusion of the Amendment Construct 1 , results in restoration of selection, insertion of an attB site, as well as a gene of interest, into the initial integration event.

D: Cre recombinase expression can then remove selection again from the transgene.

E: Amendment Construct 2 plus phiC31 integrase expression introduced to the inserted transgene results in restoration of selection, inclusion of an attP site, as well as a second gene of interest, into the transgene.

F. Expression of Cre recombinase, again results in removal of the selectable marker BAR, from the transgene. Amendment Construct 1 in combination with phiC31 Integrase expression can be used to add further cargo as in Step C. 


\section{Truncation Construct pNT1}

The construct pNT1 (Figure 5a) was used to attempt truncation of a maize B chromosome and provide a platform for further site-specific additions. The construct contains an attB, site which allows for further additions. The selectable marker BAR, which confers herbicide resistance, is flanked by lox sites so that selection can be removed. GFP under a 35S promoter was included as cargo. Finally, nearly $2 \mathrm{~kb}$ of telomere repeat arrays from WY82 (Yu et al. 2006) was cloned into the construct just inside the Right Border of the T-DNA. 


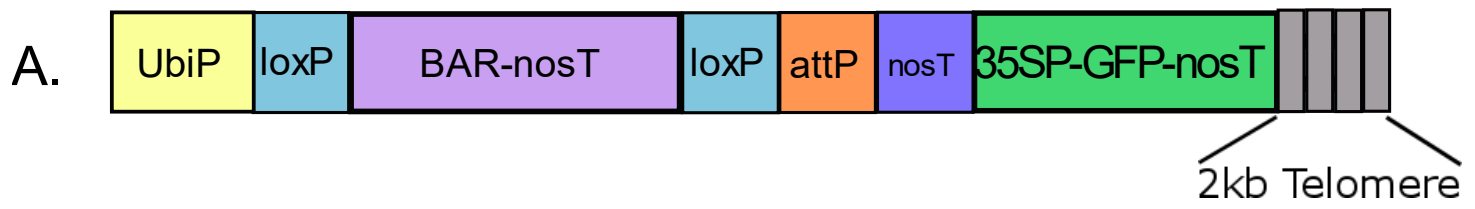

\begin{tabular}{|l|l|l|l|l|}
\hline UbiP & loxP & BAR-nosT & loxP & attP \\
\hline
\end{tabular}

\section{Figure 5: pNT1 and PZPGLP1 Construct Maps}

A: The truncation construct pNT1 is to be used as a platform for site-specific modification using Amendment Constructs 1 and 2. The construct contains the selectable marker, bialaphos resistance (BAR), driven by a Ubiquitin Promoter (UbiP) and expression terminated by a Nopaline Synthase Terminator sequence (nosT). BAR-nosT are surrounded by loxP sides oriented such that recombination using Cre recombinase will result in excision of intervening DNA. An attP site follows for integration of DNA using Amendment Construct 1 in conjunction with phiC31 Integrase. The nosT sequence following the attP site is to prevent any expression through the attP site. Green Fluorescent Protein gene (GFP) driven by a 35S cauliflower mosaic virus promoter sequence and expression terminated by a nosT. $2 \mathrm{~kb}$ of telomere sequences are at the terminal end of pNT1 to attempt to truncate whatever chromosome the construct integrates into via telomere-mediated chromosomal truncation. 


\section{Amendment Construct 1}

The system for amending the insertion site revolves around the use of two constructs whose alternating attP and attB sites allow for sequential site-specific integration into the genome at the original transgenic insertion site. The first of these constructs, Amendment Construct 1, contains an attP site that allows for site-specific integration using PhiC31 integrase, at the corresponding attB site in the integrated transgene. After site-specific integration of Amendment Construct 1 , the integration site will have an additional attP site for further integration using Amendment Construct 2.

\section{Amendment Construct 2}

The second construct in the amendable system is Amendment Construct 2. Amendment Construct 2 has the exact same composition as Amendment Construct 1, except that it contains an attB site for integration instead of attP. The attB site allows for Amendment Construct 2 to add more cargo, in a site-specific manner, to the amended transgenic integration site, which now contains an attP site. After site-specific integration of Amendment Construct 2, the integration site will have an additional attB site for further integration using Amendment Construct 1. 


\section{Materials and Methods}

\section{Construct Design}

\section{Truncation Construct pNT1}

The backbone for the truncation construct was designed by Nat Graham in a PZP202 Agrobacterium-competent vector, meaning the cassette is flanked by Left Border (LB) and Right Border (RB) sequences (see transformation below). This initial construct consists of a maize Ubiquitin Promoter driving BAR expression. Expression is terminated by a nosT and the BAR-nosT had been made Cre-removable by flanking that DNA segment with loxP sites. Following nosT-loxP is an attP site that will be utilized for site-specific addition by phiC31 Integrase. Resistance in this vector is to Spectinomycin. This construct is referred to as pPZPGLP1 and is displayed in Figure 5b. pPZPGLP1 will be used as an alternative site for site-specific addition and experiments will be described using this construct later on in the chapter.

35S-GFP-nosT was next cloned into pPZPGLP1 from the construct WY9.

eGFP expression driven by a 35S promoter as cargo on the truncation construct would not only serve as simple cargo for initial transformation but allow for screening of transformants via fluorescence. Created by a former member of the lab (Weichang Yu), WY9 contains 35S-GFP-nosT, which was cloned into the construct pPZPGLP1. 35S-GFP-nosT fragment from WY9, was amplified with the primers WY9cutFAsc1-WY9cutRAsc1. These primers also contained Asc1 sites that added these sites to the amplified fragment. This allowed for restriction digest 
of the 35S-GFP-nosT fragment with Asc1. pPZPGLP1 was digested with Asc1 and then treated with Antarctic phosphatase to ensure the plasmid did not reanneal. The restriction digested 35S-GFP-nosT fragment was cloned into the Asc1 site of pPZPGLP1 using T4 DNA Ligase. Ligations were transformed into top10 electrocompetent cells using electroporator and protocol. Electroporated DNA was transferred into $15 \mathrm{~mL}$ culture tubes containing $900 \mathrm{uL}$ of SOC media and were shaken at $37 \mathrm{C}$ for $1 \mathrm{~h} .50 \mathrm{ul}$ of media was then plated onto LB plates containing $100 \mathrm{mg} / \mathrm{mL}$ Spectinomycin. Colonies were screened using colony PCR (Sambrook et al. 1989) with the primers M13F-BARNOSSEQ3 (Appendix 2). Colonies that were positive for the 35S-GFP-nosT insert were transferred to LB liquid culture containing $100 \mathrm{mg} / \mathrm{ml}$ spectinomycin and placed into a 37C shaker overnight. Plasmid DNA extraction was performed using (Machery-Nagel plasmid kit). DNA concentration was determined using a Nanodrop Spectrophotometer. These PCR positive DNA samples were then checked for the correct insert by restriction digest with EcoRV. Positive events displayed the correct dropout fragment size. These events were labeled WY9intoPZP. The WY9intoPZP plasmid was then sequenced to check for positive events in the correct orientation. WY9intoPZP 5.3 was selected for correct sequence and orientation.

A Nopaline Synthase Termination sequence (nos Terminator) was then added immediately after the attP site to prevent any possible expression from the ubiquitin promoter through to the cargo. The nos terminator used was synthesized using IDT GBLOCK services, allowing compatible sites to be added to either side of the sequence for easy cloning into WY9PZP. Cloning of the nos 
terminator was performed using restriction cloning. Nos Terminator GBLOCK is Sbf1-nosT-Sbf1. WY9intoPZP 5.3 was digested with Sbf1, as well as the nosT GBLOCK. The cut nosT was then cloned into WY9intoPZP 5.3 using T4 DNA Ligase. Positive transformants were screened using PCR, restriction digest, and sequencing. Clone WY9PZPNOS 1.4 was chosen by the criterion of sequence integrity to be used for further cloning. 


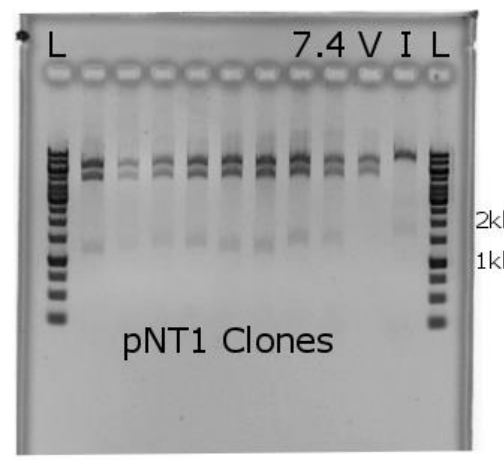

A.

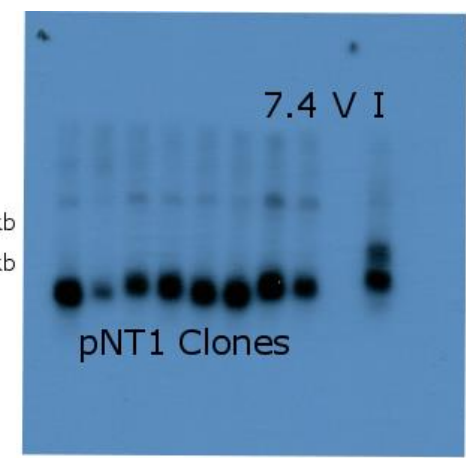

B.

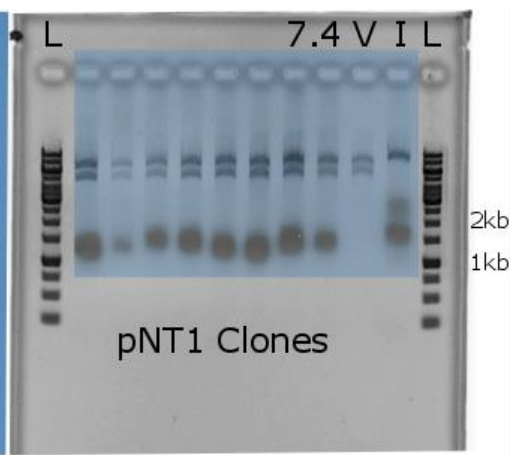

C.

Figure 6: Restriction Digest and Southern Hybridization of Telomere-Containing

\section{Clones}

A: Positively identified clones from Southern colony hybridization were grown and restriction digested by Pacl and Scal enzymes. Successful digestion results in 3 bands in positive clones: $6.9 \mathrm{~kb}$ backbone, $5 \mathrm{~kb}$ pNT1 construct, $2.5 \mathrm{~kb}$ telomere dropout. 7.4 is the pNT1 clone chosen for transformation into maize. $V$ represents the vector sequence 1.4 , I represents the insert plasmid from which telomere was derived, WY82, and $L$ designates ladder lanes that use GeneRuler $1 \mathrm{~kb}$ ladder.

B: Southern Hybridization with telomere probe of the restriction digest described in part A. Labels represent the same as described in part A.

C: Overlay of Southern Hybridization from part B onto gel image of the restriction digest in part A. 


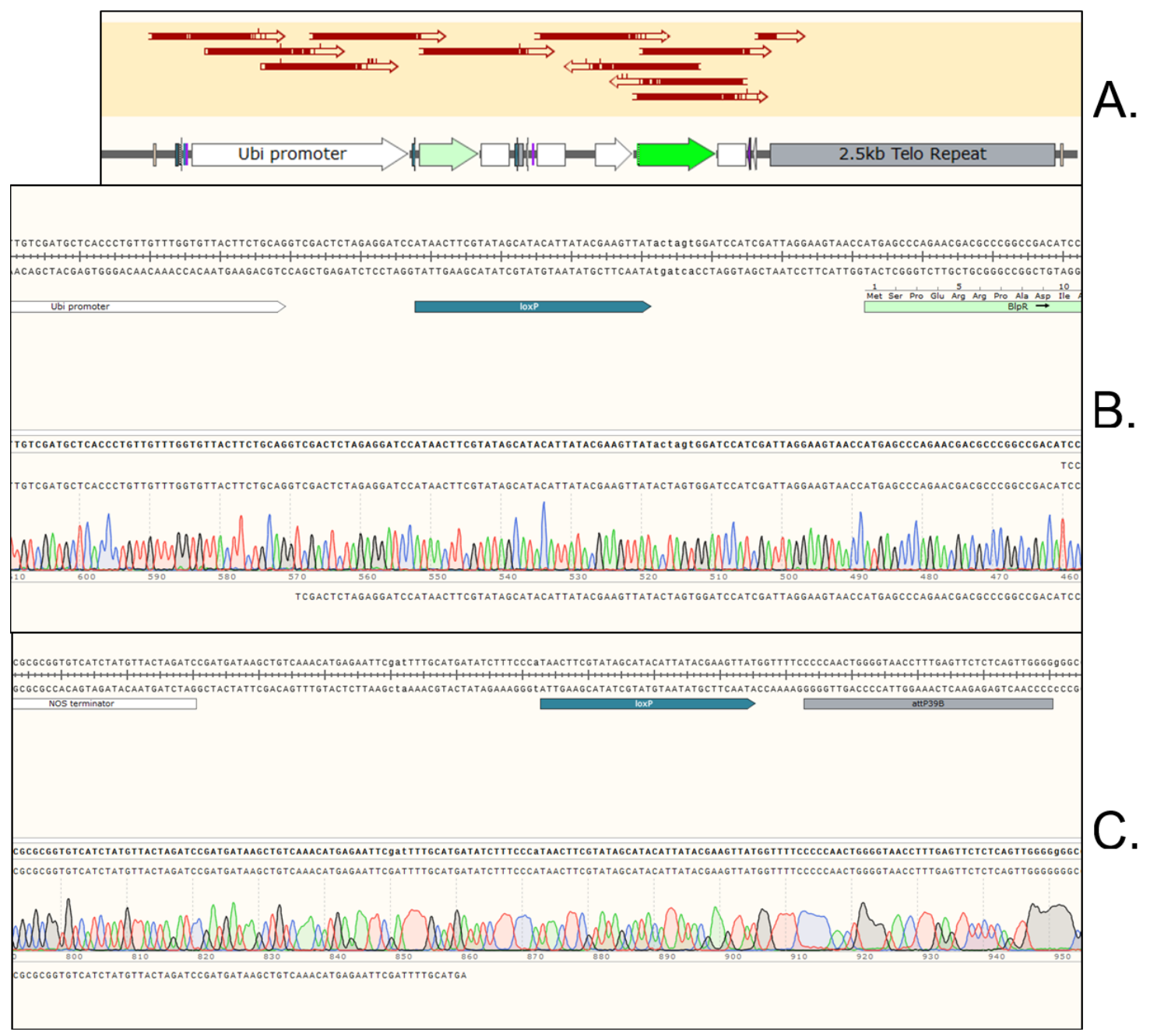

Figure 7: Sequencing of pNT1 Construct

Part A: Alignment of all sequencing performed to check the integrity of pNT1. Red bars indicate alignments of sequencing to the plasmid.

Part B: Sequencing of the first loxP site, which lies between the UbiP and BAR, and alignment to plasmid sequence.

Part C: Sequencing and alignment to second loxP site and the attP site to the pNT1 plasmid sequence. 
Telomere arrays were added from the construct WY82 to WY9PZPNOS 1.4. WY82 was created by a former member of the lab for telomere truncation (Yu et al. 2006). It contains $2.5 \mathrm{~kb}$ of Arabidopsis telomere sequence. Using the cloning protocol described (Swyers et al. 2016), $2 \mathrm{~kb}$ of telomere sequence was placed into WY9PZPNOS 1.4, just inside of the Right Border sequence. Stbl 4 cells were used to stabilize the telomere repeat. Appendix 2 describes cloning of telomere DNA into a construct in detail. Positive transformants were screened using a colony hybridization and Southern Blot analysis (Swyers et al. 2016) (Figure 6). To determine the size of the telomere repeat, a digest was performed, followed by a Southern Hybridization using a telomere probe. PCR and Sanger sequencing were performed to confirm the integrity of the construct (Figure 7). pNT1 was chosen for use by sequence integrity and the size of the telomere repeat that had been cloned into the construct. Final pNT1 in stbl4 cells was grown in LB media at 30C over 3 days. Plasmid extraction was performed on the cultured pNT1. This plasmid DNA was transformed into the EHA105 Agrobacterium cells. Selection for pNT1 in Agrobacterium cells was performed using Streptomycin/Spectinomycin/Rifampicin. Agrobacterium-mediated transformation was then performed with pNT1. 
A.

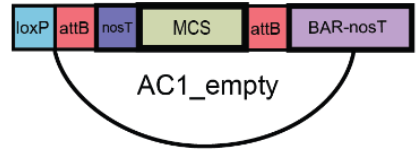

B.

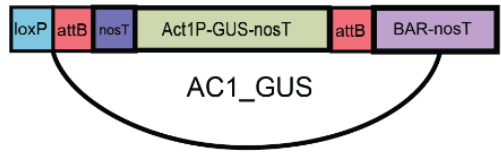

C.

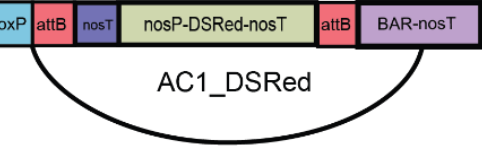

D. \begin{tabular}{|l|l|l|l|l|l|}
\hline LxP & attB & nost & UbiP-Integrase-nosT & attB & BAR-nosT \\
\hline
\end{tabular}

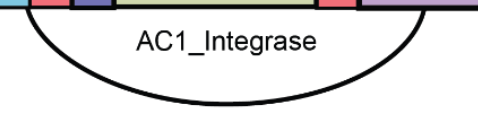

\begin{tabular}{ll|l|l|l|l|l|}
\hline ExP & attB & nost & 2Kb Telomere & attB & BAR-nosT \\
\hline
\end{tabular}

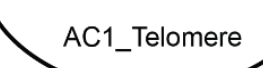

F.

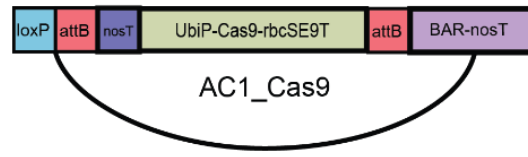

G. \begin{tabular}{l|l|l|l|l|l|}
\hline loxP & attB & nost & lat52P-Apt1-35ST & attB & BAR-nosT \\
\hline
\end{tabular}

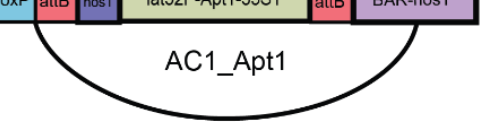

H. \begin{tabular}{|l|l|l|l|l|l|}
\hline LoxP & attB & nosT & Act1P-Bxb1-nosT & attB & BAR-nos T \\
\hline
\end{tabular}

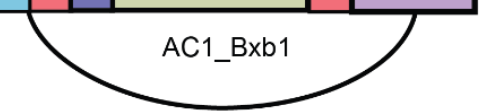

I. \begin{tabular}{|l|l|l|l|l|l|}
\hline LoxP & attB & nost & Act1P-FLP-nosT & attB & BAR-nosT \\
\hline
\end{tabular}
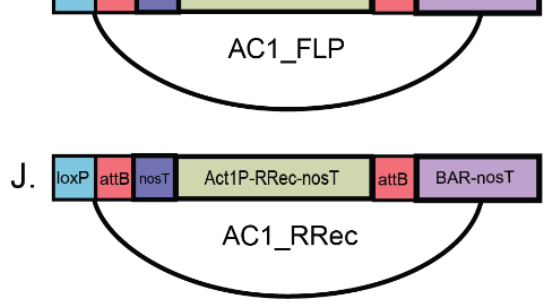

Figure 8: Amendment Construct1 and Its Cargo

A. The construct AC1empty was synthesized and sequenced by GenScript. The construct contains all the necessary sites and machinery for amending the initial truncation construct pNT1 as outlined in Figure 4. This version of amendment construct 1 contains an empty multiple cloning site (MCS), which will be used for the cloning of cargo into the plasmid such as in parts C-G.

B. AC1Gus is Amendment Construct 1 that has a $\beta$ glucurinadase gene driven by a rice Actin 1 promoter and terminated by a nos terminator cloned into the MCS of the amendment construct. C: AC1DSRed is Amendment Construct 1 with a DSRed expression cassette cloned into the MCS where cargo should be included. DSRed expression is driven by a nos promoter and terminated by a nos terminator.

D. The construct AC1Integrase contains a phiC31 Integrase expression cassette in the MCS of Amendment Construct 1. Expression of phiC31 Integrase is driven by a maize ubiquitin promoter and expression terminated by a nos terminator.

E. AC1Telomere contains $2 \mathrm{~kb}$ of telomere sequences cloned into the cargo MCS of Amendment Construct 1. Subcloning of telomere sequences into the amendment construct was performed by Intact Genomics. Integrity was confirmed by PCR and restriction digest by Intact Genomics.

F. This version of amendment construct 1 contains a Cas9 expression cassette cloned into the MCS. Cas9 expression is driven by a maize ubiquitin promoter and terminated by a rubisco subunit e9 terminator.

G. An apt1 expression cassette was cloned into the MCS of Amendment Construct 1 by GeneWiz to create AC1 apt1. The expression cassette contains apt1 driven by a lat52 pollen-specific promoter and expression terminated by a 35s cauliflower mosaic virus terminator.

H. AC1Bxb1 contains a Bxb1 Integrase expression cassette, driven by the rice Actin1 promoter and expression terminated by a nos terminator.

I. The construct AC1FLP has a FLPase expression cassette cloned into the MCS of amendment construct 1.

$\mathbf{J}$. This variant of amendment construct 1 contains an R-recombinase expression cassette. $\mathrm{R}$ recombinase expression is driven by a rice Actin1 promoter and expression terminated by a nos 
A.

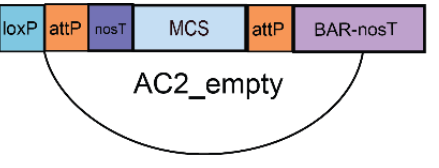

B.

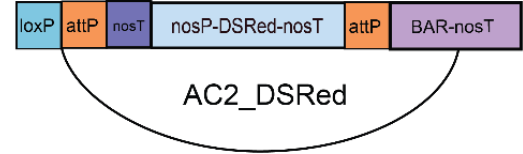

C.

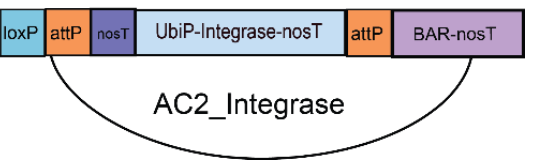

Figure 9: Amendment Construct 2 and Its Cargo A. AC2empty is analogous to AC1empty except with attP sites in place of attB sites. AC2empty is the second construct needed for the amendment portion of the system detailed in Figure 4 to work. This construct also contains a MCS for the cloning of cargo such as in parts $\mathrm{H}$ and $\mathrm{I}$.

B. AC2DSRed is Amendment Construct 2 with a DSRed expression cassette cloned into the MCS by GeneWiz. This expression cassette is exactly the same as that contained in AC1DSRed in part A. C: AC2Integrase contains a phiC31 Integrase expression cassette cloned into the MCS of amendment construct 2 by GeneWiz. The expression cassette is analogous to that contained in AC1Integrase in part B. 


\section{Amendment Constructs}

AC1empty/AC2empty: AC1empty and AC2empty were synthesized by GenScript and each placed into a pBlueScript backbone. Amendment Construct 1 and 2 with empty cargo multiple cloning sites (MCS) were designed with 18 unique cut sites in the middle of each construct to allow for cloning of cargo into each. AC1empty, outlined in Figures 8A and 9A, was designed so that BAR becomes active only upon phiC31 Integrase-mediated recombination between the correct attB site and the attP site located on in the genome. BAR in the construct is promoterless and is followed by a nos termination sequence. The attB site immediately preceding BAR must be the site of recombination for expression of BAR, which allows for selection. Following BAR-nosT is the plasmid backbone, which will be carried into the genomic site by successful recombination. A lox site is located after the plasmid backbone, which, in combination with the lox site in the genomic transgene, will allow for removal of BAR-nosT along with the plasmid backbone in a later cross. Following this lox site is a second attB site that will allow or further modification using Amendment Construct 2. As outlined previously, recombination between this attB site and the genomic attP site will not result in activation of BAR expression and therefore will not be selected. Finally, cargo is placed into the multiple cloning site following the second attB site. AC2empty is designed in an analogous fashion to AC1empty except that AC2empty contains attP sites in place of the attB sites in AC1empty. Figures 9 and 10 show layouts of amendment construct 1 and 2 variants, respectively. AC1empty and AC2empty were used to develop a number of cargo 
carrying constructs (Figure 8B-J, Figure 9B-C). Maps of the following constructs are displayed in Appendix 1.

AC1GUS: AC1GUS is AC1empty with a GUS expression cassette cloned into the MCS of AC1empty. The GUS expression cassette was cloned into AC1empty by GenScript and sequenced by GenScript to confirm the integrity of the construct. AC1GUS would allow for screening of positive transformants by GUS staining in addition to selection by BAR.

AC1DsRed: The construct AC1DsRed is a version of AC1empty that contains a DsRed expression cassette in the MCS of AC1empty. The DsRed expression cassette was cloned into AC1empty by GeneWiz and sequencing was performed by GeneWiz. AC1DsRed allows for screening by fluorescence of DsRed in addition to selection by BAR.

AC1Integrase: A cassette for expression of phiC31 Integrase was also cloned into $\mathrm{AC} 1 \mathrm{empty}$ to create the construct $\mathrm{AC} 1$ Integrase. Cloning and sequencing was performed by GeneWiz to ensure correct subcloning of the expression cassette into AC1empty. This construct allows for site-specific integration of phiC31 Integrase as well as providing the recombinase necessary to amend itself into the genomic attP site.

AC1Telomere: This version of amendment construct 1 contains $2 \mathrm{~kb}$ of telomere sequences in the MCS of AC1empty. Subcloning of telomere into AC1empty was accomplished by Intact Genomics. AC1Telomere could allow for site-specific 
truncation via telomere-mediated truncation when it undergoes recombination into a genomic attP site.

AC1Cas9: AC1Cas9 has a Cas9 expression cassette cloned into the MCS of AC1empty. This version of amendment construct 1 will allow for expression of Cas9 to be included into a genome-located attP site. Subcloning of the Cas9 expression cassette into AC1empty was performed by GeneWiz who also sequenced the resulting construct for integrity.

AC1apt1: In order to create a system of pollen selection for minichromosomes, an apt1 expression cassette was cloned into AC1empty. The expression cassette was synthesized and sequenced by GenScript and subcloned into AC1empty by Intact Genomics. Inclusion of apt1 into a minichromosome would allow for future tests of pollen selection in an apt1 mutant background.

AC1Bxb1: This construct contains an expression cassette for the recombinase Bxb1 Integrase. This recombinase has been shown to function in plants and may be useful for future iterations of the amenable system. The Bxb1 expression cassette was synthesized and sequenced by GeneWiz.

AC1FLP: AC1FLP contains an expression cassette for the recombinase FLPase. FLPase has been shown to function in maize in the dissertation by Jon Cody and operates in a similar manner to Cre recombinase. This FLPase expression cassette was synthesized and sequenced by GeneWiz. 
AC1Rrec: An expression cassette for R-recombinase was synthesized and sequenced by GeneWiz and placed into amendment construct 1. This recombinase has been shown to function in the dissertation by Jon Cody.

AC2DsRed: This version of amendment construct 2 has a DsRed expression cassette cloned into the MCS of AC2empty. Subcloning and sequencing was performed by GeneWiz. This construct is analogous to AC1DsRed but would allow for amendment to an attB site.

AC2Integrase: AC2Integrase is analogous to AC1 Integrase except that in this version the phiC31 expression cassette has been cloned into AC2empty. Cloning and sequencing were performed by GeneWiz. 


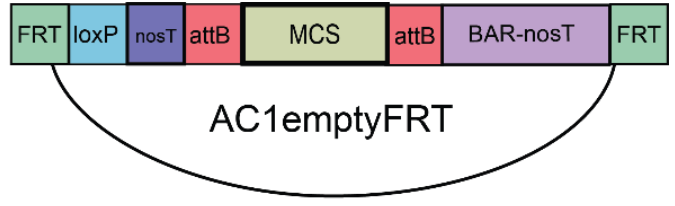

Figure 10: Amendment Constructs

Flanked by FRT Sites

Agrobacterium-mediated transformation is thought to deliver linear molecules into the genome of the host plant. In order for successful amendment to an att site in the genome using phiC31 Integrase, the

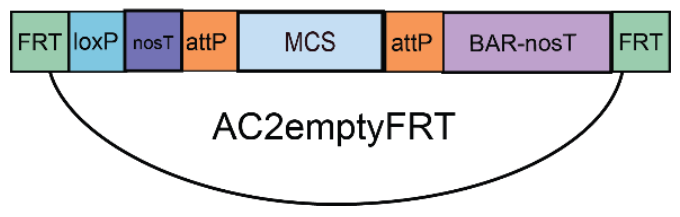
amendment construct must be circular. To facilitate circularization during Agrobacterium transformation, amendment constructs were designed that are flanked by FRT sites, which, when acted upon by FLPase, results in their circularization. For circularization to occur expression of phiC31 Integrase and FLPase must be present in the genome with the target att site. 
AC1EFRT/AC2EFRT: These versions of amendment constructs 1 and 2 are entirely similar in sequence to AC1empty and AC2empty, respectively, except for 2 major differences. The first difference is that these constructs contain FRT sites that are located preceding the lox site on one end and proceeding BAR-nosT on the other. These two constructs are outlined in Figure 10. Agrobacterium is thought to deliver TDNA into cells as a linear molecule, though circularization is possible (Singer 2012). In order for amendment to result in a desirable product, the amendment construct must be a circle when phiC31 Integrase recombines the complementary sites of the amendment construct and the genomic att site. When exposed to FLP recombinase, these constructs will circularize because of the orientation of the FRT sites, which will allow for desired amendment via recombination from phiC31 Integrase. Secondly, these constructs have been subcloned into an Agrobacterium-compatible vector to allow for transformation to be performed via Agrobacterium. Synthesis of these constructs was performed by GenScript and subcloning performed by GeneWiz. They have been sequence verified by GeneWiz. 


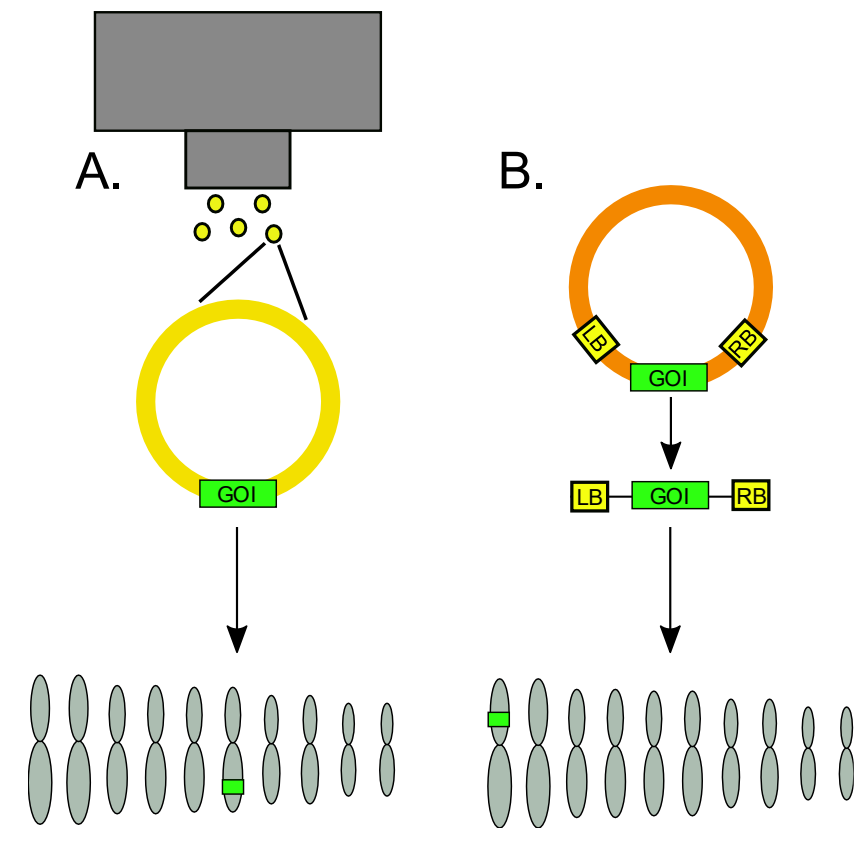

Figure 11: Maize

Transformation Methods

Part A: Biolistic Transformation.

DNA-covered gold particles are

propelled at high speed via

pressurized helium, into immature

maize embryos or callus.

Transgene integration happens randomly.

Part B: Agrobacterium-mediated Transformation: The transgene is placed inbetween the Left Border (LB) and Right Border (RB) of the Tumor inducing Plasmid ( $\mathrm{Ti}$ plasmid) of the Agrobacterium. The transgene is then delivered by the bacterium into immature maize embyros where it can integrate randomly into the genome. 


\section{Transformation}

\section{Materials}

Agrobacterium-mediated transformation of immature maize embryos

There are two methods regularly used for maize transformation, Agrobacterium transformation and particle bombardment (Figure 11). The first method utilizes the natural ability of a soil bacteria, Agrobacterium tumefaciens, to integrate some of its DNA into a plant cell's nucleus. In the wild, Agrobacterium introduces DNA from its tumor inducing plasmid (Ti Plasmid) into the plant cell, which causes that plant to form a tumor upon which bacteria feed. The genes from the Ti plasmid that are inserted into the plant genome lie between two DNA sequences known as the left border (LB) and right border (RB). Scientists have removed those genes causing tumor formation from the plasmid so that DNA of interest can be inserted between the LB and RB of a disarmed Ti plasmid. This modified Ti plasmid can be transferred into an Agrobacterium strain and this strain used to transform plant cells with DNA of interest.

In maize, the tissues used for Agrobacterium transformation are immature embryos from a genotype that forms type 2 callus. The maize genotypes used for transformation in this study was Hi II A carrying B chromosomes and Hi II B (Armstrong et al. 1991). To generate embryos for transformation, Hi II A and B are crossed together to give plants that show some heterosis. Hi II displays $100 \%$ type- 2 callus initiation. Type 2 callus is friable, which makes selection for positive events easier, and allows for making clonal events to ensure propagation of positive transformants. In contrast to type 2 callus, type 1 callus is 
characterized by being non-friable, with a hard exterior that is not easy to break apart and is resistant to selection (Ishida et al. 2007).

Transformation of the truncation construct pNT1 was accomplished using Agrobacterium-mediated transformation following Vega, J., et al., 2008. This method was used because of its propensity to result in only single copy insertions of relatively large transgenes (Ishida et al. 2007). A second advantage of Agrobacterium transformation over biolistic delivery is that biolistic transgene delivery can frequently result in transgene rearrangements while Agrobacterium transformation does not (Ishida et al. 2007).

Tissue used for Agrobacterium transformation were embryos from Hi II A+B chromosome $x \mathrm{Hi}$ II B and F1 selfs of the previous cross. Embryos were harvested 10-12 days after pollination.

\section{Biolistic Transformation}

The second major method used for maize transformation is particle bombardment. This method of transformation is accomplished by shooting DNAcoated gold particles at high speeds into maize tissue using high pressure helium. Particle bombardment can be performed on either immature embryos or callus tissue in maize (Frame et al. 2000).

Amendment construct 1 with its various cargo and attBDSRed were transformed using particle bombardment. Particle bombardment was used as it keeps the amendment constructs in a circular conformation allowing for favorable integration into pNT1 by phiC31 Integrase. Target tissue containing insertion 
sites were obtained from plants, which were screened to contain the Cre-reduced pNT1 transgene or Cre-reduced pPZPGLP1 transgene without the Cre transgene. Cre-reduced refers to insertion sites that have been exposed to Cre recombinase to remove the selectable marker (BAR). Target tissues were also obtained from plants containing the Cre-reduced pPZPGLP1 transgene and a phiC31 Integrase expressing transgene. Those screened plants were selfed, sibbed, or crossed to Hi-II B plants for embryos to use for bombardment. Embryos and calli from the previously mentioned crosses were the target tissue for bombardment of amendment construct 1 or attBDSRed. Particle bombardment was performed using a protocol based on Frame et al. 2000 .

\section{Screening Transformants}

\section{$\mathrm{FISH}$}

Protocols outlined in Appendices 3 and 4. Fluorescence in Situ Hybridization (FISH) was used to screen transgenic events. FISH works by labeling target DNA via a nick translation (McCaw et al. 2016a). Target DNA for FISH is metaphase nuclei obtained from root tips (McCaw et al. 2016b). This technique was used to screen positive pNT1 transformants to determine the location of the transgene.

The construct WY9PZPNOS 1.4, which is pNT1 before telomere had been cloned into the plasmid, was used to generate a FISH probe for transgene localization. This probe was labeled by TexasRed fluorophore.

Other FISH probes used to screen transgenic events and deduce the location of the insertion, included CentC centromeric repeat probe, TAG repeat probe, and 
NOR probe that binds to the nucleolar organizing region. All of these probes were labeled with AlexaFluor green.

\section{Genomic DNA Extraction}

Genomic DNA was obtained from both leaf tissue and callus tissue using either of two methods: Urea extraction (Leach et al. 2016), and by Qiagen DNeasy Plant Mini Kit (catalog \# 69106).

\section{PCR Screening}

PCR screening was performed using JumpStart ${ }^{\mathrm{TM}}$ REDTaq $^{\circledR}$ DNA Polymerase (catalog \# D8187-250UN). Primers used are located in Table 2. 


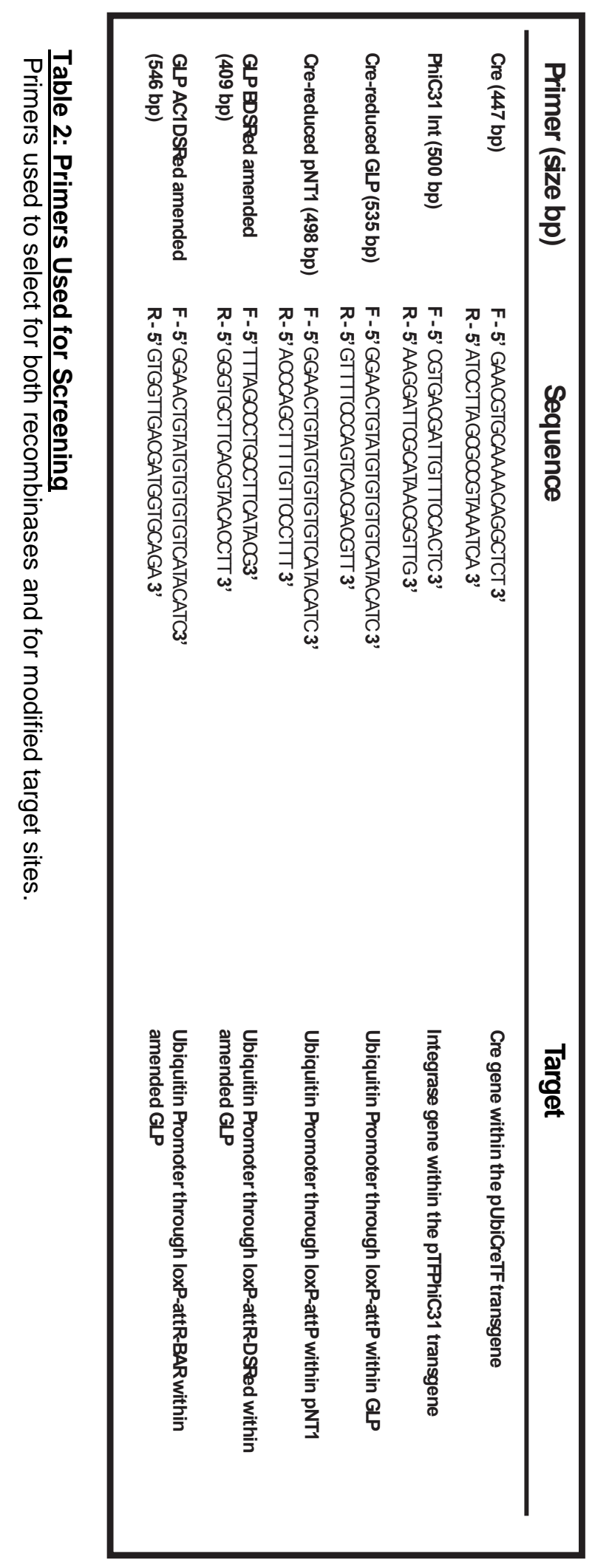




\section{Results}

The truncation construct pNT1 was transformed into $7510 \mathrm{Hi}$ II embryos containing B chromosomes via Agrobacterium-mediated transformation. 26 transgenic events have been obtained, none containing a minichromosome, but one having a B chromosome insertion termed pNT1 (event 11) shown in Figure 12. The insertion of the pNT1 transgene into the B chromosome was chosen for further use, as there could be some utility obtained from the B chromosome location. It can serve as a proof of concept for targeted transformation of an independent chromosome. The ability of the B chromosome to nondisjoin means that, from this single insertion event, I can find progeny containing more than once copy of this B chromosome insertion. This may be a useful demonstration of the ability to increase transgene copy via the B chromosome. The lack of essential genes on the B chromosome also makes this insertion site particularly useful. In the future, this B chromosome transgene insertion might be truncated or the construct pNT1 could be used in additional transformations to recover a truncated B chromosome. 


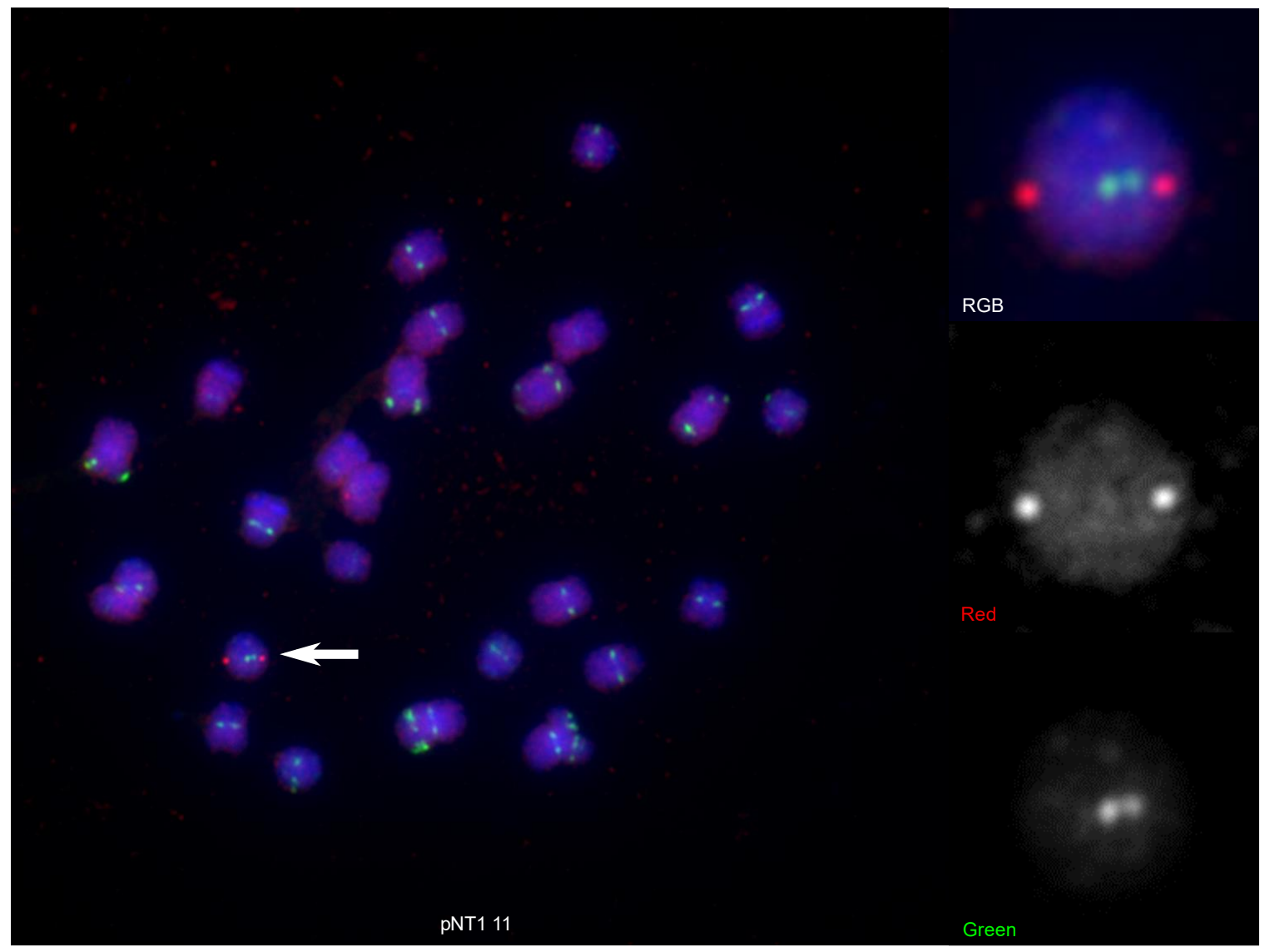

\section{Figure 12: pNT1 Inserted into B Chromosome}

FISH image of pNT1 transgenic event 11. The arrow indicates the location of the B chromosome insertion of pNT1. The insets are expanded images of that B chromosome in the RGB, Red, and Green channels. In red is the single gene FISH probe derived from the pNT1 plasmid minus telomere and labeled with TexasRed. In green are TAG and CentC repeat probes labeled in AlexaFluor green. The strong TAG signal on the long arm as well as the shape of the chromosome indicate that it is a B chromosome. 
This insertion site was located using FISH (Figure 12) and transgene integrity verified using PCR (Figure 13) and Sanger sequencing across the recombination sites, shown in (Figure 14). 


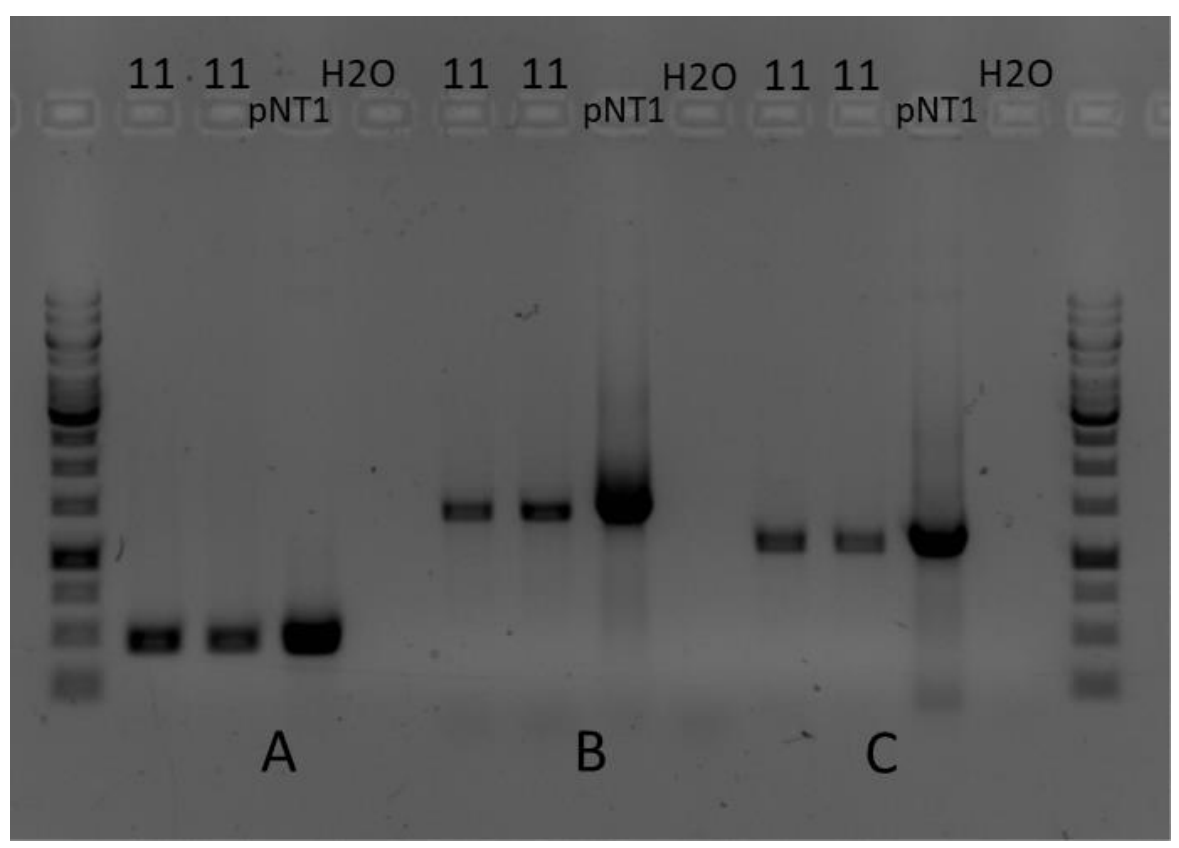

Figure 13: Integrity Check of pNT1 11

$A, B$, and $C$ indicate 3 PCR primer sets used to test the integrity of pNT1 11, which is the $B$ chromosome insertion. PCR primer set $A$ amplifies a product of 473 basepairs that includes the attP site and the second loxP site. PCR primer set $B$ amplifies a product of 1433 basepairs, which includes the first loxP site as well as BAR-nosT. Primer set $\mathrm{C}$ amplifies a product of 1134 base pairs. 


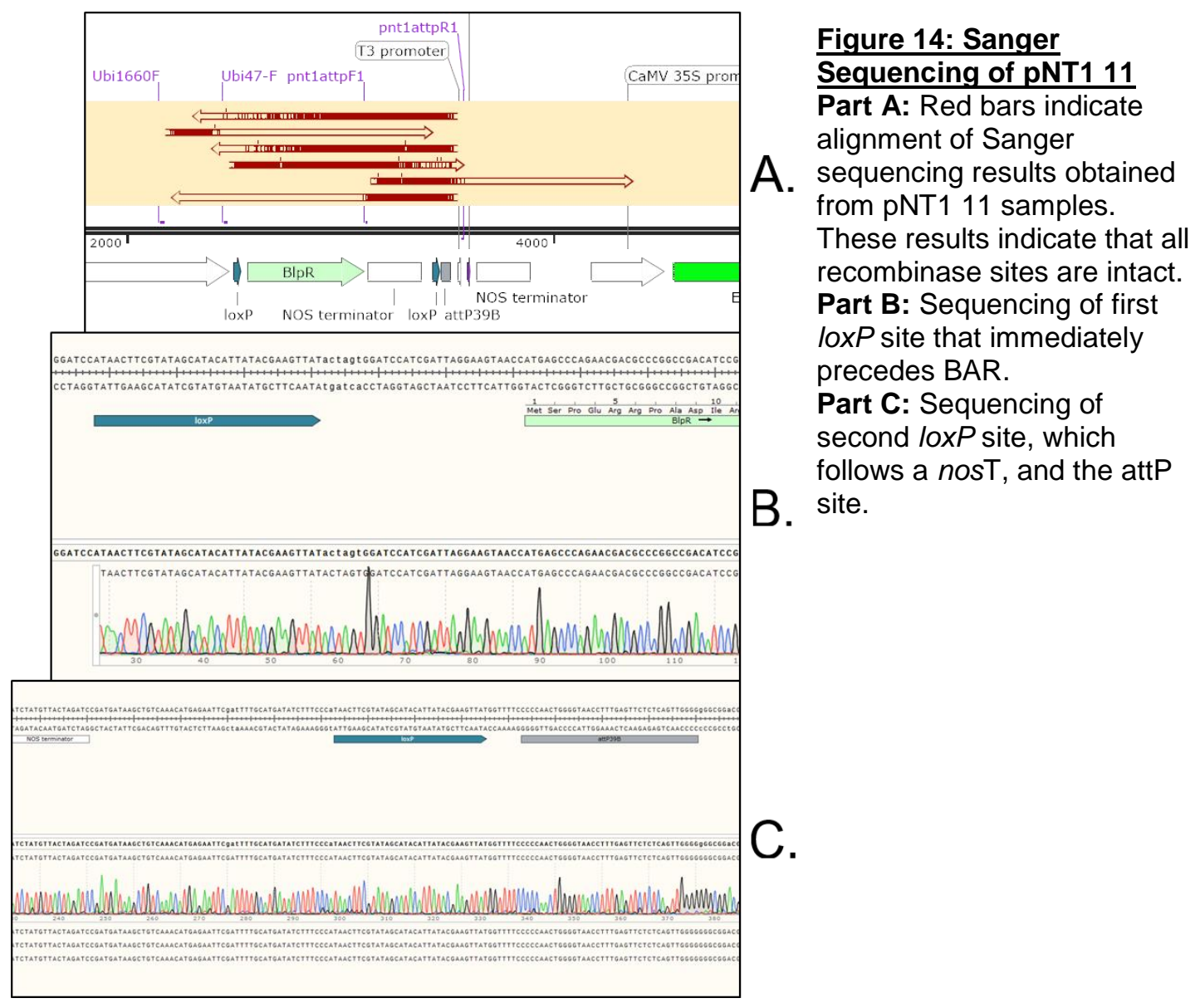

Maize plants containing pNT1 11 were crossed to a maize line expressing Cre recombinase (Cre expression line created by Robert Gaeta). The goal of crossing pNT1 11 to Cre recombinase is the removal of BAR from the integrated 
transgene pNT1 as illustrated in Figure 14. The resulting progeny were screened using PCR for the removal of BAR from the pNT1 site on the B chromosome, example shown in Figure 15 and primers used listed on Table 2. 
\begin{tabular}{|l|l|l|l|l|l|l|}
\hline AbiP & loxP & attP & nost & B5SP-GFP-nosT & & \\
\hline
\end{tabular}

\begin{tabular}{|l|l|l|}
\hline BbiP & IoxP & attP \\
\hline
\end{tabular}

Figure 15: Cre Recombinase Removal of Selection

A: Cre-reduced pNT1. Exposure to Cre-recombinase removes BAR selection.

B: Cre-reduced pPZPGLP1. Exposure to Cre-recombinases removes BAR selection from the transgene. 


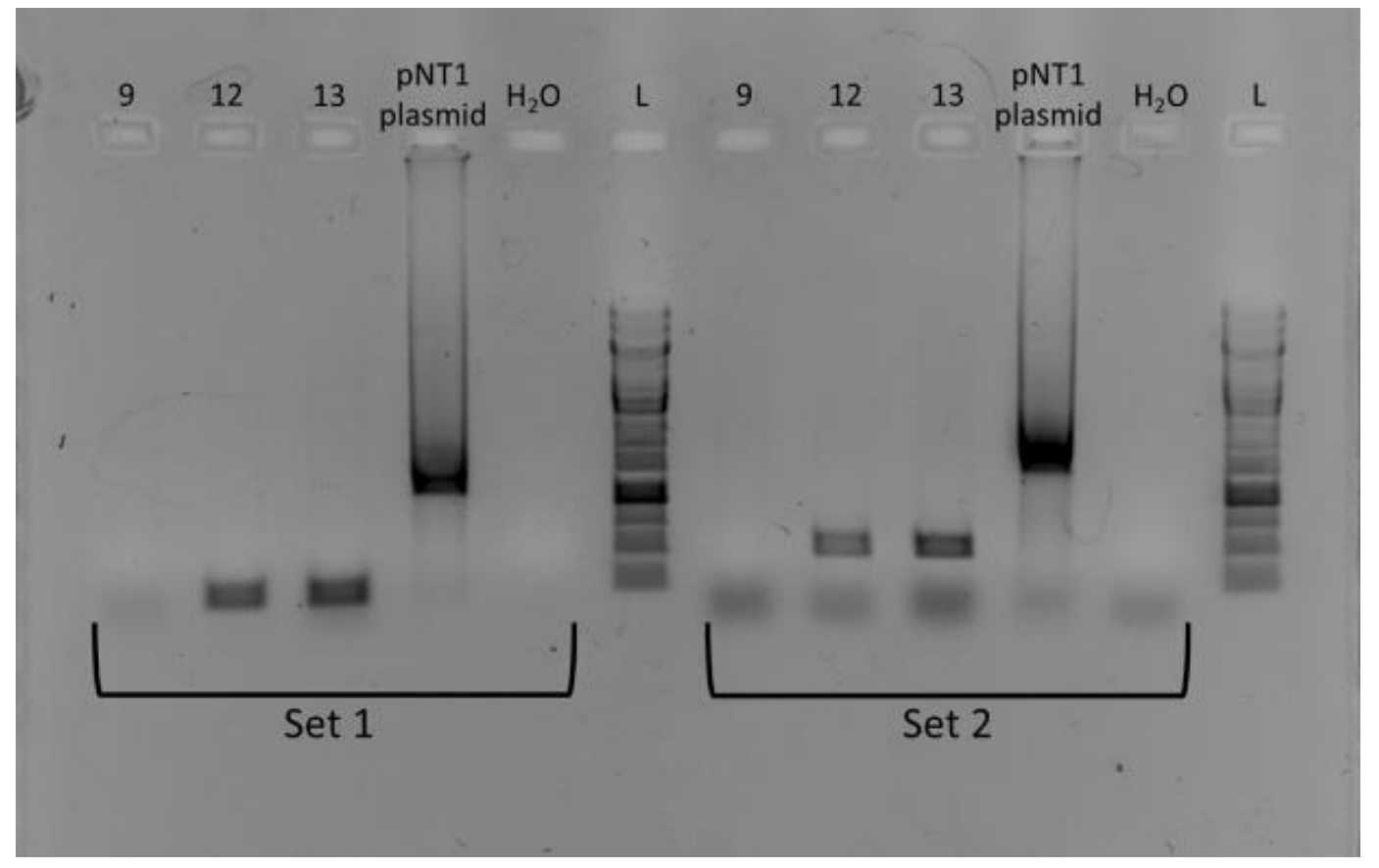

Figure 16: pNT1/Cre Gel

Primer set 1 band size is 1134 base pairs with BAR present and 200 base pairs when BAR is absent. Primer set 2 amplifies a 1433 base pair region with BAR present and a 499 base pair region when BAR is absent. Samples 12 and 13 display correct band size if BAR has been removed from pNT1. Sample 9 lacks the pNT1 transgene, so it shows no banding. pNT1 plasmid control displays band size if BAR has not been removed by Cre recombinase. 
Several plants that were shown by PCR to be Cre-reduced where checked by FISH to confirm that the PCR primers were working, which is shown in Figures 17 and 18. Figures 17 and 18 are derived from the same plants as samples 9 and 13 from Figure 16 respectively. 


\section{Figure 17: FISH Confirmation Control of PCR Selection of pNT1 $11 \times$ Cre}

FISH image of pNT1 transgenic event 11 crossed by Cre that was indicated by PCR to not contain the pNT1 transgene. In red is the single gene FISH probe derived from the pNT1 plasmid minus telomere and labeled with TexasRed. In green are TAG and CentC repeat probes labeled in AlexaFluor green. The Cre transgene is highlighted by the pNT1 probe as well and shows in the picture because of a large amount of sequence homology between pNT1 and the Cre transgenes. pNT1 is not present in the FISH image which corroborates the PCR result shown in Figure 16. 


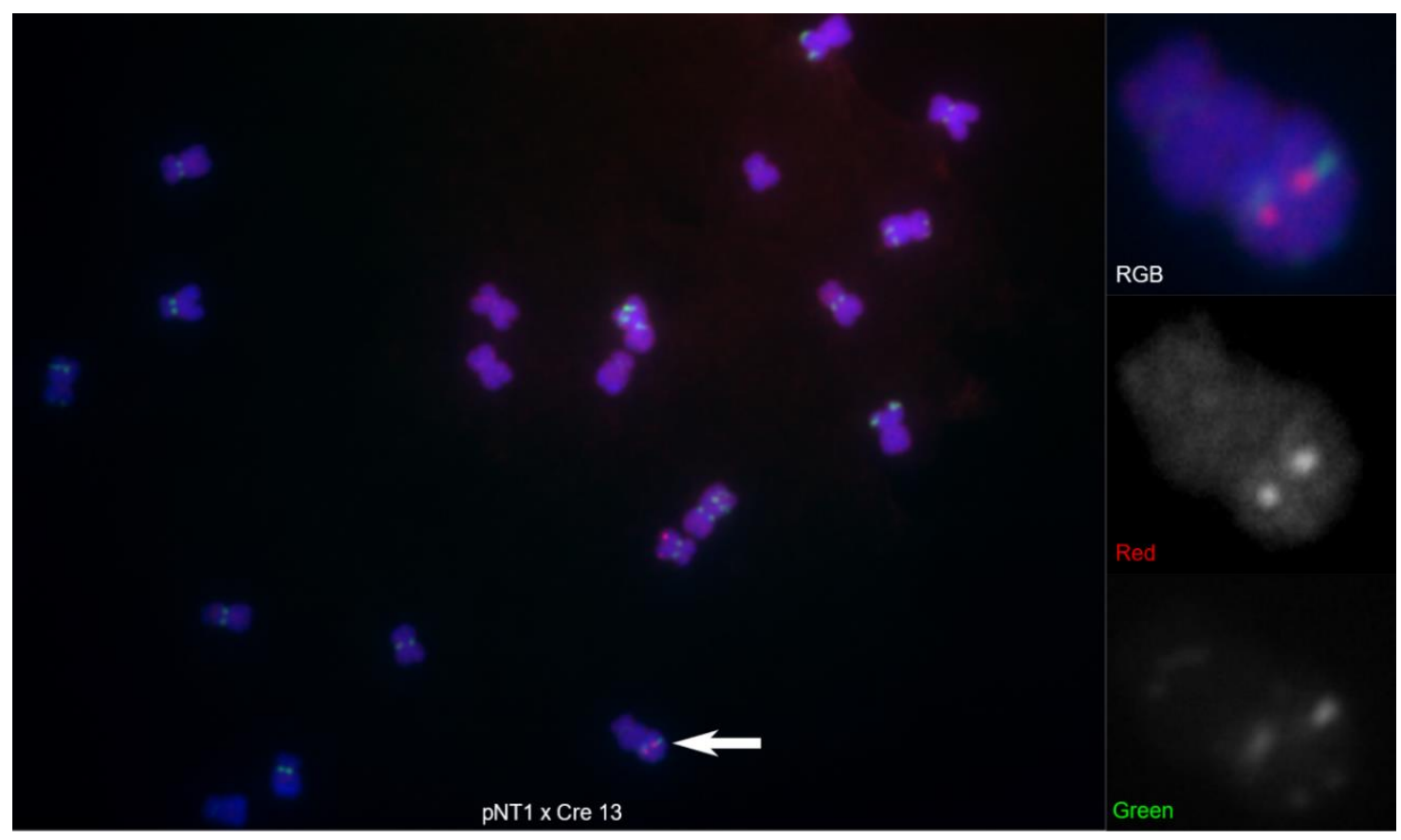

\section{Figure 18: B Inserted pNT1 Crossed by Cre}

FISH image of pNT1 transgenic event 11 crossed into a Cre expressing line. The arrow indicates the location of the B chromosome insertion of pNT1. The insets are expanded images of that B chromosome in the RGB, Red, and Green channels. In red is the single gene FISH probe derived from the pNT1 plasmid minus telomere and labeled with TexasRed. In green are TAG and CentC repeat probes labeled in AlexaFluor green. The strong TAG signal on the long arm as well as the shape of the chromosome indicate that it 
Progeny containing the Cre-reduced pNT1 site were crossed to Hi II plants. The Cre-recombinase expressing construct contains a non-removable BAR selectable marker, so this transgene must be crossed away from pNT1 11 to allow for herbicide selection of subsequent amendment events. Progeny from this cross were screened by PCR to contain Cre-reduced pNT1 and lacking the Cre expressing construct, as this construct contains BAR selection. Sanger sequencing of the relevant recombinase sites in a Cre-reduced pNT1 11 individual was performed to check integrity recombinase sites, which is shown in Figure 19. The same steps outlined above were performed for the alternative target site pPZPGLP1 as well to isolate the Cre-reduced target site from the Cre expressing transgene. 


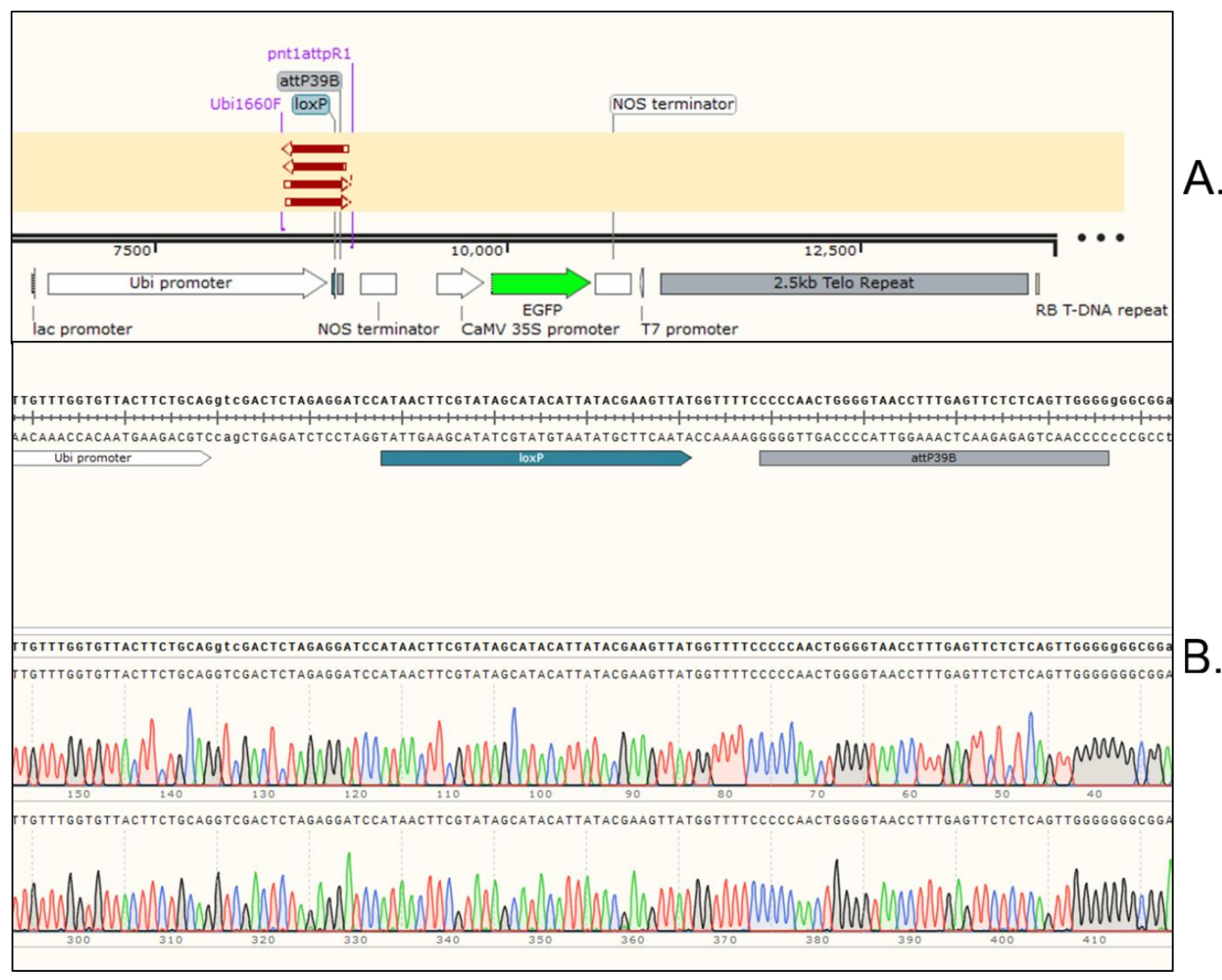

Figure 19: Sequencing of Cre-reduced pNT1

Sequencing was performed on 2 Cre-reduced pNT1 plants.

Part A: The arrows in part A indicate the alignment of the sequencing data to the Crereduced transgene.

Part B: Part B shows the raw sequencing alignment over the recombinase sites, demonstrating their integrity and that BAR-nosT has been removed so the transgene has indeed been reduced by Cre recombinase. 


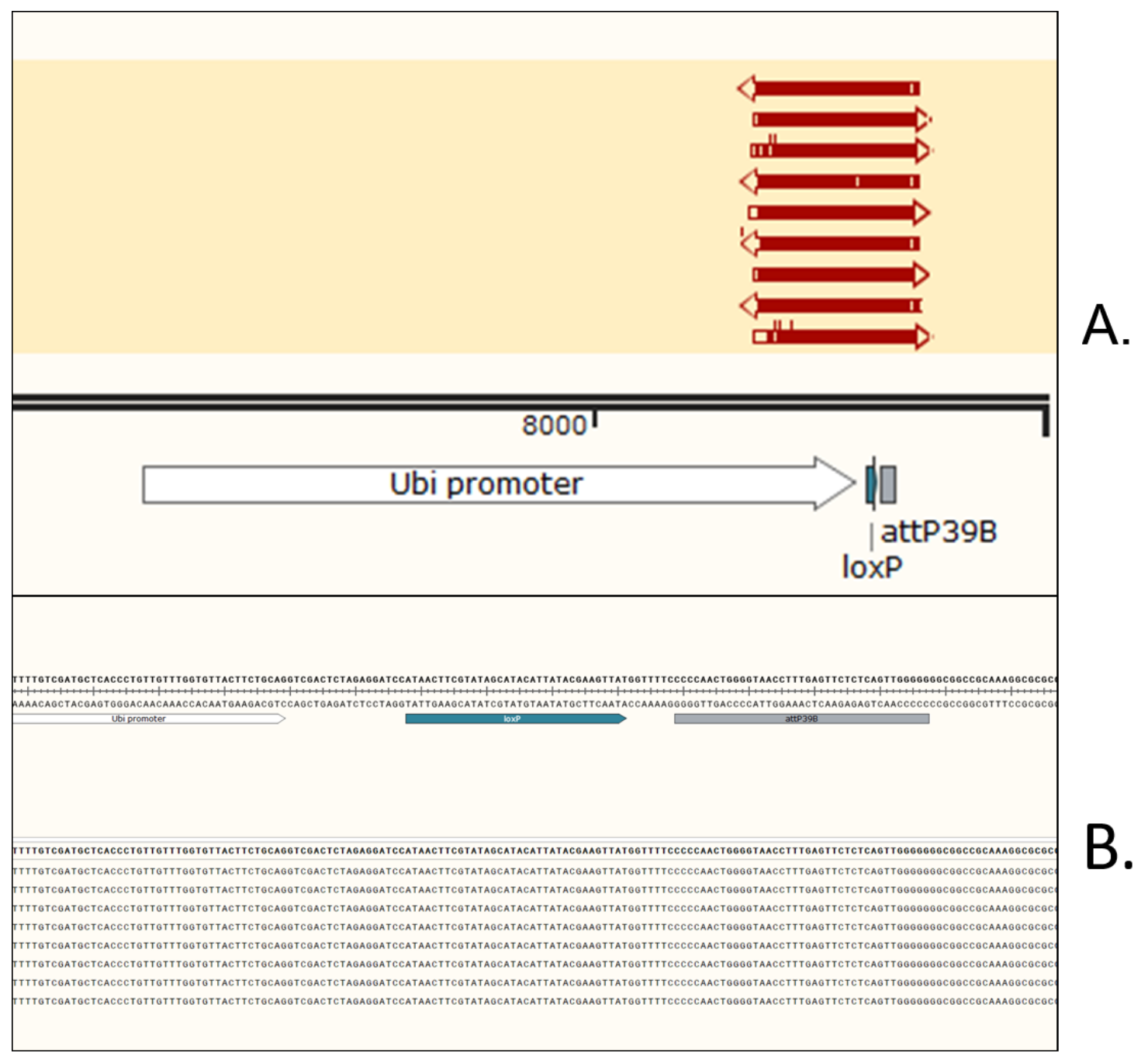

Figure 20: Sequencing of Cre-reduced PPZPGLP1

Sequencing was performed on Cre-reduced pPZPGLP1 plants.

Part A: The arrows in part A indicate the alignment of the sequencing data to the Crereduced transgene.

Part B: Part B shows the raw sequencing alignment over the recombinase sites, demonstrating their integrity and that BAR-nosT has been removed so the transgene has indeed been reduced by Cre recombinase. 
Those individuals containing the Cre-reduced pNT1 11 and no Cre were selfed/sibbed and embryos/calli used for transformation. Transformation was performed by particle bombardment of embryos/callus with several different versions of amendment construct 1. AC1DsRed, AC1Telo, which are Amendment Construct 1 containing a DSRed expression cassette in the multiple cloning site of the amendment construct, and amendment construct 1 containing 2kb Telomere DNA, respectively, were cobombarded with a plasmid expressing phiC31 Integrase. AC1Integrase was bombarded both alone and with an Integrase-expressing plasmid, as expression of phiC31 Integrase is driven by a ubiquitin promoter, which should enable expression and integration of this version of amendment construct 1. To control for site-specific integration being construct dependent, a second construct (Figure 21b) attBDSRed was used for cobombardment as well. To date, cobombardment of Amendment Construct 1, or attBDSRed, together with phiC31 Integrase has not resulted in detection of a positive transformant, either by tissue culture or PCR. 

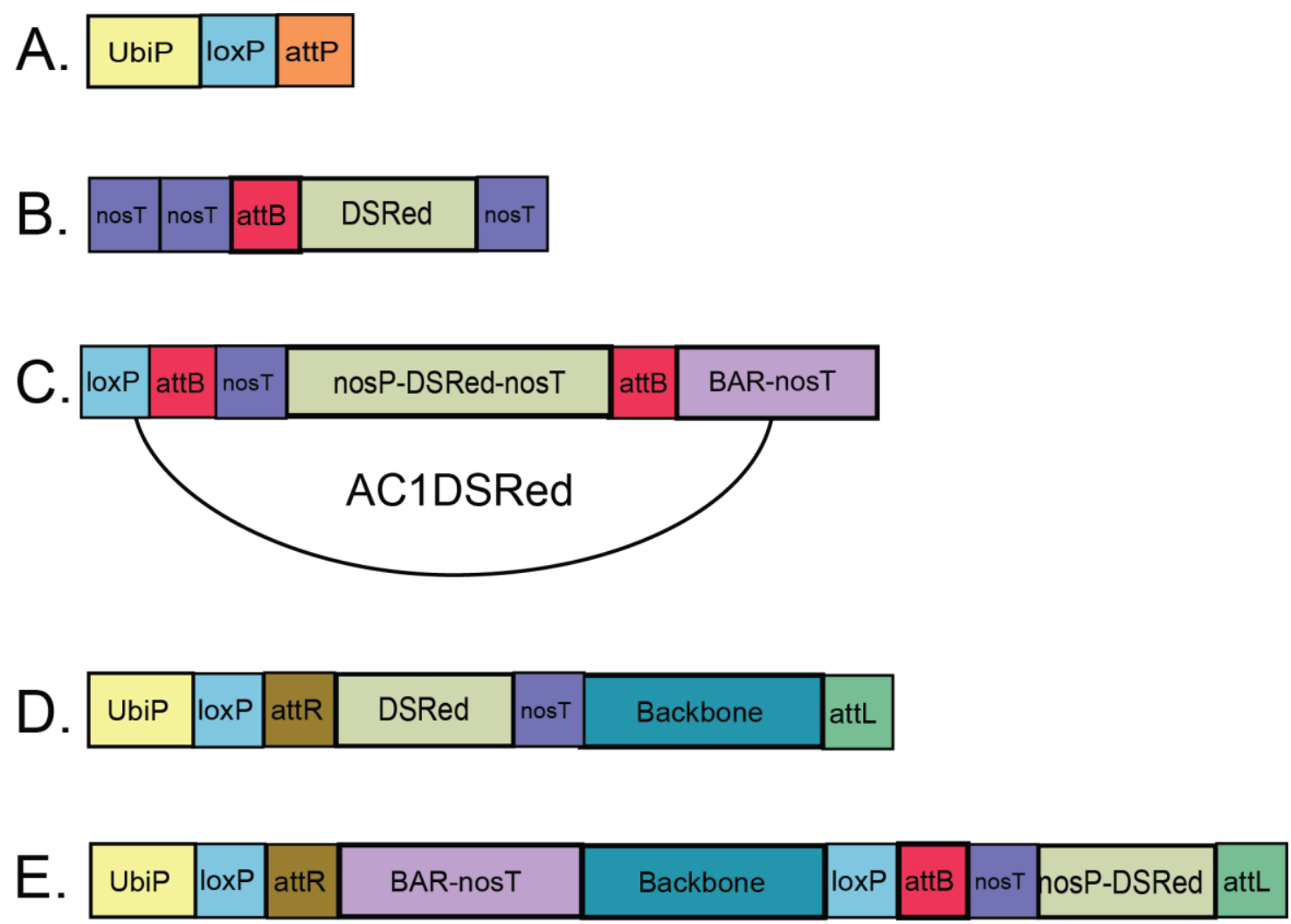

Figure 21: pPZPGLP1 Targeting Experiment Components

A: Cre-reduced pPZPGLP1 is shown with a Ubiquitin promoter followed by a loxP site and an attP site. The attP site can be targeted for site-specific amendment by the constructs in parts $\mathrm{B}$ and $\mathrm{C}$, in conjunction with phiC31 Integrase.

B: attBDsRed construct to be used to demonstrate site-specific integration. Upon sitespecific integration DsRed expression is activated allowing for visualization.

C: AC1DsRed as previously described is used to test whether site-specific integration is possible using an amendment construct. Site-specific integration allows for PCR amplification and sequencing of a Ubiquitin promoter-loxP-attR-BAR junction not present in the target site before amendment.

D: pPZPGLP1 after site-specific integration of attBDsRed.

E: pPZPGLP1 after site-specific integration of AC1DsRed. 


\section{pPZPGLP1 as a Proof of Concept}

As an alternative to amending pNT1 11, a second construct of similar makeup was used to demonstrate the ability of the amendment system to work. This construct, pPZPGLP1, was transformed into maize and has been used in the lab to test the efficacy of using phiC31 Integrase in maize (Figure 5b). Like pNT1, pPZPGLP1 consists of a Ubiquitin Promoter driving Bialaphos expression with expression terminated by a NOS terminator. The Bar-NOST cassette is flanked by loxP sites, which, upon being acted upon by Cre recombinase, will excise the Bar-NOST cassette. Following the above steps, an attP site has been placed in a normal "A" chromosome to allow for site-specific modification using phiC31 Integrase. pPZPGLP1 has been previously crossed to a Cre recombinase expressing line which resulted in the removal of BAR selection (Figure 20). Subsequent crosses have eliminated the Cre-recombinase expressing construct from the PPZPGLP1 background allowing for potential amendment via a cobombardment of Amendment Construct 1 and phiC31 Integrase. Cre-reduced pPZPGLP1 has also been crossed to a line containing a phiC31 Integrase expression transgene (Figure 2d). This recombinase expression cassette contains a non-removable Bialaphos gene, which renders it useless for stacking; however, it is useful for demonstrating the feasibility of using phiC31 Integrase to stack onto the PPZPGLP1 transgene. As performed with pNT1, Cre-reduced pPZPGLP1 embryos were cobombarded with several versions of Amendment Construct 1 or attBDsRed, and a phiC31 Integrase expression cassette. 
Cobombardment experiments have not resulted in any evidence of site-specific integration to date.

In a second set of experiments Cre-reduced pPZPGLP1/ phiC31 Integrase embryos were bombarded by both AC1DsRed and attBDsRed. AC1DsRed and attBDsRed are described in detail below. Images were taken, DNA extraction performed on transformed embryos, followed by PCR and Sanger sequencing. The results of this set of experiments show definitive evidence of site-specific integration into the pPZPGLP1 site by both constructs (Figures 22 and 23).

\section{AC1DsRed}

AC1DsRed consists of Amendment Construct 1 and has a DsRed gene driven by a Nopaline Synthase promoter, all followed by a Nopaline Synthase termination sequence to stop transcription and form a stem loop structure. These elements have been cloned into the multiple cloning site for cargo in Amendment Construct

1. The experimental function for inclusion of DsRed expression is for the ability to visually screen positive transformants by fluorescence, in addition to herbicide resistance.

\section{attBDSRed}

This construct was created in order to test for site-specific integration by phiC31 Integrase. Site-specific integration by attBDSRed into the attP site of either Crereduced pPZPGLP1 or pNT1 will cause the DsRed gene to integrate behind a Ubiquitin promoter resulting in expression of DsRed. DsRed expression allows for visual confirmation of successful site-specific integration in addition to using PCR and Sanger sequencing (Figure 21b). 


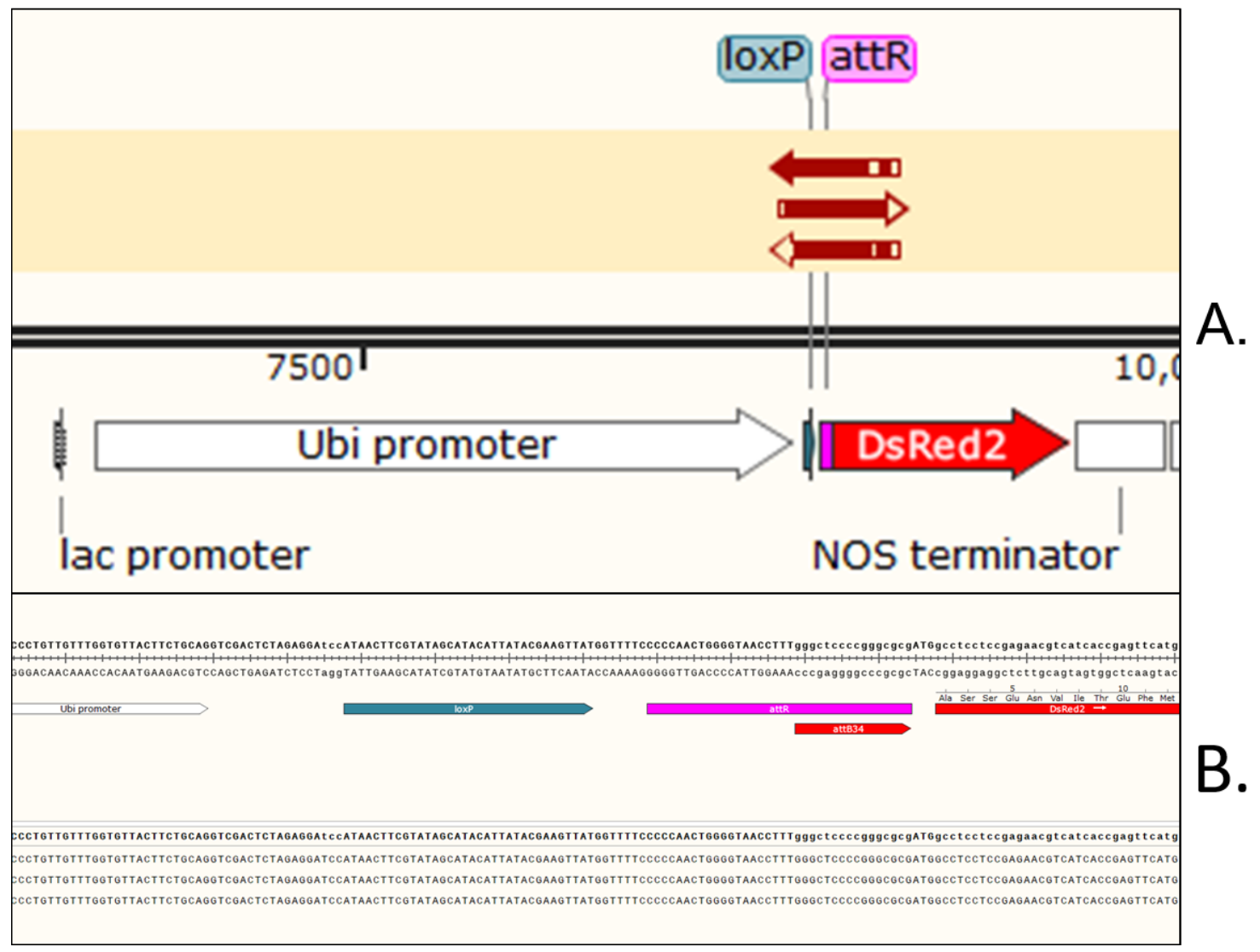

Figure 22: pPZPGLP1 attBDsRed Amendment

Sanger sequencing and alignment of sequences to the amended PPZPGLP1 target site by attBDsRed. Sequencing matches the expected result of site-specific integration as displayed in Figure 20D.

A: Ubiquitin promoter-loxP-attR-DsRed junction displayed with red arrows indicating alignment of Sanger sequencing reactions.

B: Base-pair alignment of Sanger sequencing from A indicating that site-specific integration has occurred. 


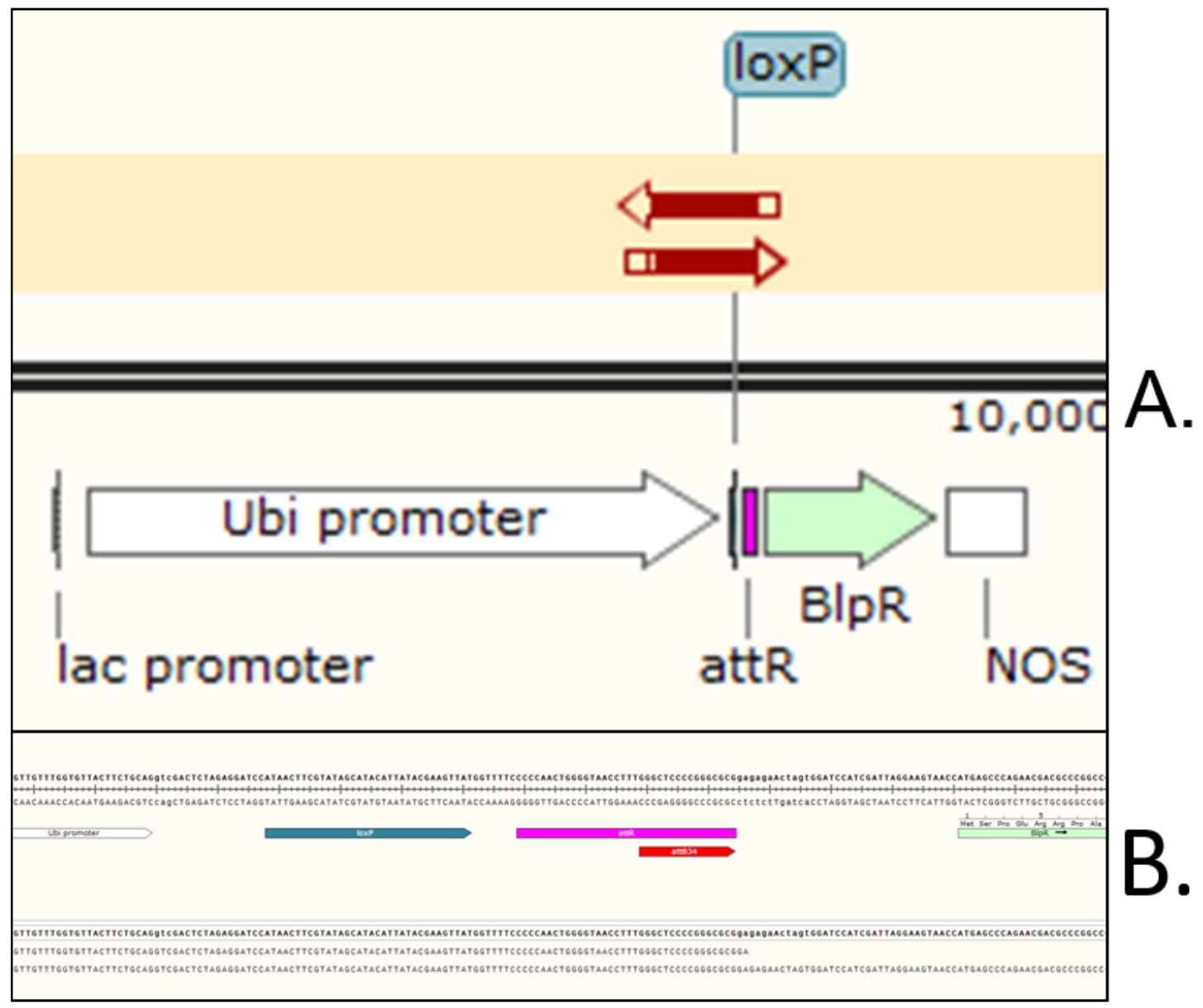

Figure 23: pPZPGLP1 AC1DsRed Amendment

Sanger sequencing and alignment to the amended pPZPGLP1 target site by AC1DsRed. Sequencing results match the expected results of site-specific integration as displayed in Figure 20E.

A: Ubiquitin promoter-loxP-attR-BAR junction displayed with red arrows indicating alignment of Sanger sequencing reactions.

B: Base-pair alignment of Sanger sequencing from A indicating that site-specific integration has occurred 


\section{Discussion}

We have successfully demonstrated that phiC31 Integrase can be used for sitespecific integration into an attP site located in the maize genome. We have also shown that Amendment Construct 1 can be utilized to achieve site-specific integration and deliver cargo to an attP site in the maize genome. From our experiments, we believe that to achieve site-specific integration requires that the target site and a phiC31 Integrase-expressing transgene be present in the target tissue. This conclusion is arrived at by the lack of evidence of site-specific integration from cobombardment of donor DNA with phiC31 Integrase into tissue containing a target site, and evidence of successful site-specific integration occurring only by bombardment of donor DNA into target tissue containing phiC31 Integrase and the target site. In regards to the efficiency of site-specific integration, we have image data (Supplemental Figure 1) from the attBDsRed targeting experiment outlined previously, as well as an analogous experiment using an attBGUS construct outlined in the dissertation by Nat Graham. From these images, we can intuit that site-specific integration seems to be occurring in several cells in a given bombarded embryo. This evidence is supported by the sequencing results from Figure 22, which together support the idea that sitespecific integration is not an extremely rare event provided the right set of circumstances (phiC31 Integrase and target site both located in target tissue). The transgene expressing phiC31 Integrase that resulted in successful site- 
specific integration contains a non-removable BAR gene. Transgenes that express phiC31 Integrase and contain removable BAR genes are being crossed to lines containing target sites such as pPZPGLP1 and pNT1. These resulting progeny will allow for regeneration of target sites (pPZPGLP1 or pNT1) amended by amendment construct 1 in tissue culture by BAR activation using the amendment system as described in Figure 4.

\section{Future Directions}

Demonstration of the ability to integrate, modify, and amend a construct are important for establishing the feasibility of the entire amendment system;

however, demonstration of amendment using amendment construct 2 is required to show that both varieties of amendment construct function. The next steps for validation that the entire system functions will be regeneration of an amended pPZPGLP1, or pNT1, and removal of BAR selection again, repeating the cross away from Cre-recombinase, isolation of Cre-reduced individuals lacking Crerecombinase in the genomic background, and finally amendment using amendment construct 2. Completion of these additional steps will validate that the entire system functions as designed. Further rounds of amendment could be made to PPZPGLP1 or the B chromosome insertion to demonstrate further gene stacking by continued rotation between amendment constructs 1 and 2 .

\section{AC1FRT, AC2FRT}

Amendment constructs must be in a circular form in order for phiC31 Integrasemediated recombination to result in integration of the amendment construct and 
cargo without the loss of DNA distal to the recombination site. A linearized form of an amendment construct recombining with a linear chromosomal insertion site would result in DNA distal to the chromosome insertion site being moved onto the linearized plasmid and vice versa for the plasmid DNA. The above interaction would result in a loss of cargo from the pNT1 site, as well as loss of the end of the chromosome. This is the reasoning for using particle bombardment to transform the amendment constructs into pTN1 plants. It is thought that integration of DNA via Agrobacterium-mediated transformation occurs via nonhomologous end joining of linear double-stranded TDNA into chromosome breaks (Singer et al. 2012). As discussed previously, for amending pNT1, amendment via linear recombination would not result in a favorable outcome. There is evidence that T DNA can form circles in the plant nucleus naturally (Singer et al. 2012), but a system to force all T DNA into circles has been designed. To allow amendment constructs to be transformed using Agrobacterium, a second set of amendment constructs has been created with FRT recombination sites flanking the amendment constructs, which are shown in Figure 9. FRT sites are acted upon by FLPase (FLP) recombinase. When exposed to FLPase, FRT containing amendment constructs should circularize, allowing for favorable recombination by phiC31 Integrase. Testing of this system requires expression of Flippase and phiC31 Integrase in the presence of the target att site, in addition to the use of the amendment constructs flanked by FRT sites. 


\section{Cas9 B chromosome}

A version of Amendment Construct 1 expressing Cas 9 exists and will be used to add Cas9 expression to the B chromosome insertion of pNT1. Secondarily, an amendment construct can be designed to carry the guides for whatever gene the researcher wishes to be modified. Having two separate versions of the amended B chromosome, one containing Cas9 expression and the other expressing the guides, allows for modification of the genome and subsequent crossing away of the guides and Cas9. One could imagine a system where a series of amended pNT1 B chromosome insertions with a variety of Cas9 guides along with Cas9 expression could be used to selectively modify the genome.

\section{Pollen Selection Using apt1}

A major issue in using a B-derived minichromosome, or B chromosome insertion for gene stacking, is that even with 2 copies of the minichromosome or B insertion inheritance of the transgene is not guaranteed. In order to remedy this predicament, a system must be created for ensuring the faithful transmission of the transgene into $100 \%$ of progeny. The aberrant pollen transmission (apt1) offers a solution through its use as a selectable marker through pollen. The apt1 gene is required for pollen tube growth in maize pollen and is homologous to SABRE and KIP proteins in Arabidopsis, which are involved in the elongation of root tips and pollen tubes (Xu and Dooner 2006). Homozygous mutants in apt1 show severe obstruction of pollen tube growth and development resulting in short and twisted pollen tubes causing near male sterility (Xu and Dooner 2006). In order to utilize this gene, apt1 has been cloned into amendment construct 1 . 
Once apt1 has been added into pNT1 11, positive transformants can be crossed into heterozygous apt1 +/- plants. Progeny from the pNT1 11 (apt1) x apt1 (+/-) cross will be backcrossed into apt1 $+/-$. The resulting progeny will then be screened for homozygous apt1 -/- individuals that contain pNT1 11 (apt1), and these seeds grown until flowering. Pollen will then be screened for demonstration of rescue by pNT1 11 (apt1). Successful demonstration shows that pollen selection of minichromosomes is viable.

\section{Babyboom and Wuschel Amendment Construct}

In order for the system described in Figure 4 to work using herbicide resistance requires that, after each amendment, herbicide selection is removed. This removal requires that the target att site be exposed to a recombinase (in this case Cre), which adds several months to the process of amending, as one must follow a cross to the recombinase with a cross away from the recombinase. To reduce the time between amendments a possible solution is to change how the

amended att site is selected. Overexpression of the maize genes Bbm and Wus2 allow for callus formation in maize lines that are normally recalcitrant to callus formation and transformation (Lowe et al. 2016). The genes Bbm and Wus2 express morphogenic regulators, which when overexpressed, have been known to produce embryo-like structures on explants (Lowe et al. 2016). These two genes can be used to select for positive transformants by callus formation, as only transformed cells will proliferate and form calli on regeneration media (Lowe et al. 2016). This effect could be utilized to reduce the time between amendments by removing the requirement for Cre-mediated BAR removal. 
Inclusion of a rab17P-Cre-nosT cassette and a Bbm-Wus2 expression cassette just downstream of BAR-nosT in an amendment construct would allow for selection by callus formation and Cre-mediated removal by dessication of calli. The proposed system is outlined in Figure 24. 


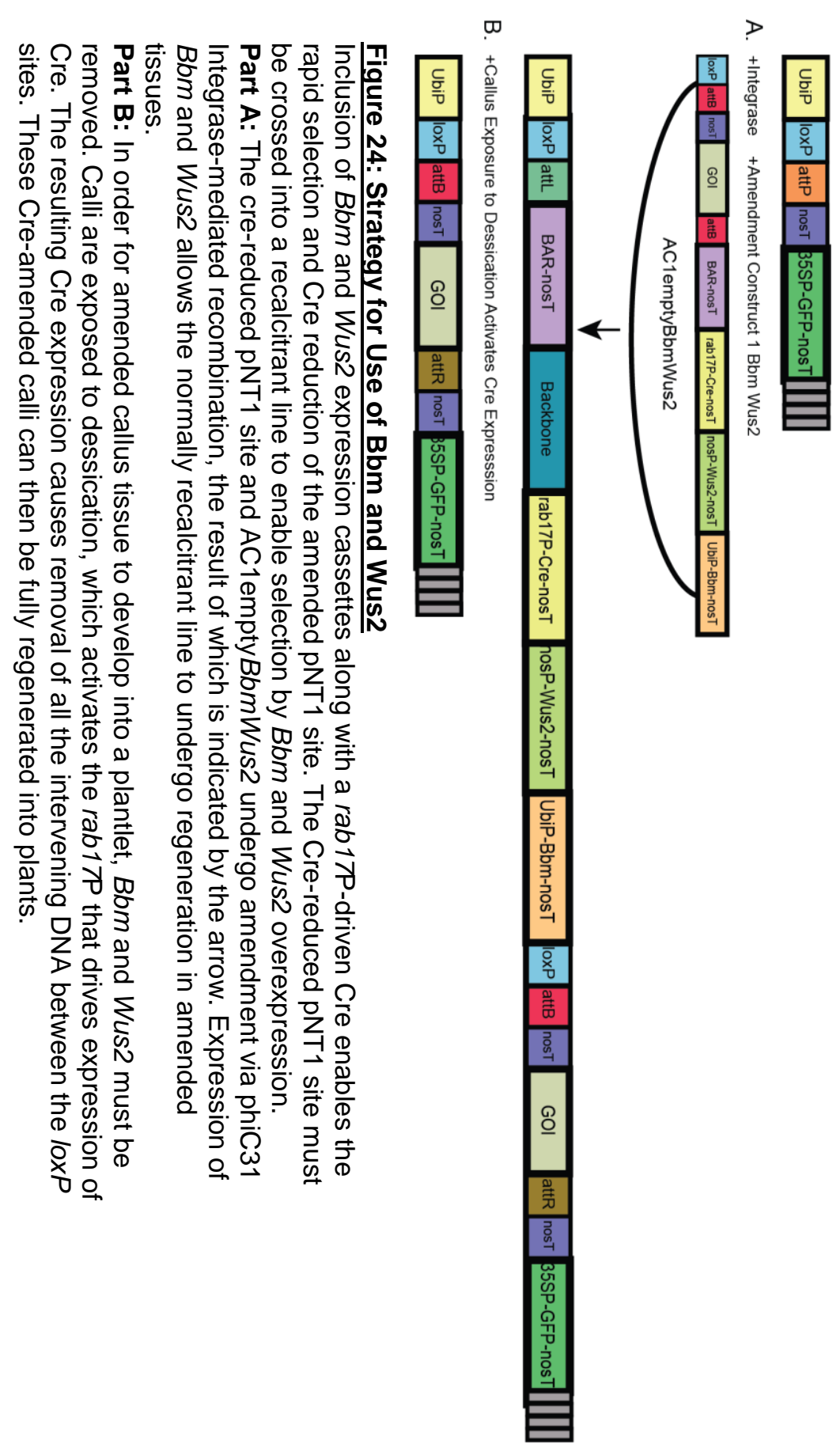


Excision of $\mathrm{Bbm}$ and Wus2 is required in order for healthy plantlets to be obtained as full regeneration under Bbm and Wus2 results in abnormal plant phenotypes (Lowe, K., et al. 2016). The inclusion of a Cre expression cassette driven by an inducible promoter, rab1ד, enables the removal of $B b m$ and Wus2. Dessication activates the rab17P, which drives Cre expression. Cre expression would then cause removal of BAR-nosT, rab17P-Cre, and the Bbm-Wus2 expression cassette. This Cre-mediated removal would result in the same product from this modified amendment construct as would occur in the original amendment constructs outlined previously.

\section{AC1Telo}

AC1Telo is the version of amendment construct 1 which contains $2 \mathrm{~kb}$ of Arabidopsis telomere DNA, derived from WY82, cloned into the multiple cloning site of amendment construct 1 . This construct was designed with the goal of testing the ability to site-specifically truncate a chromosome using telomeremediated chromosomal truncation. Truncation possibilities are outlined in Figure 24. 


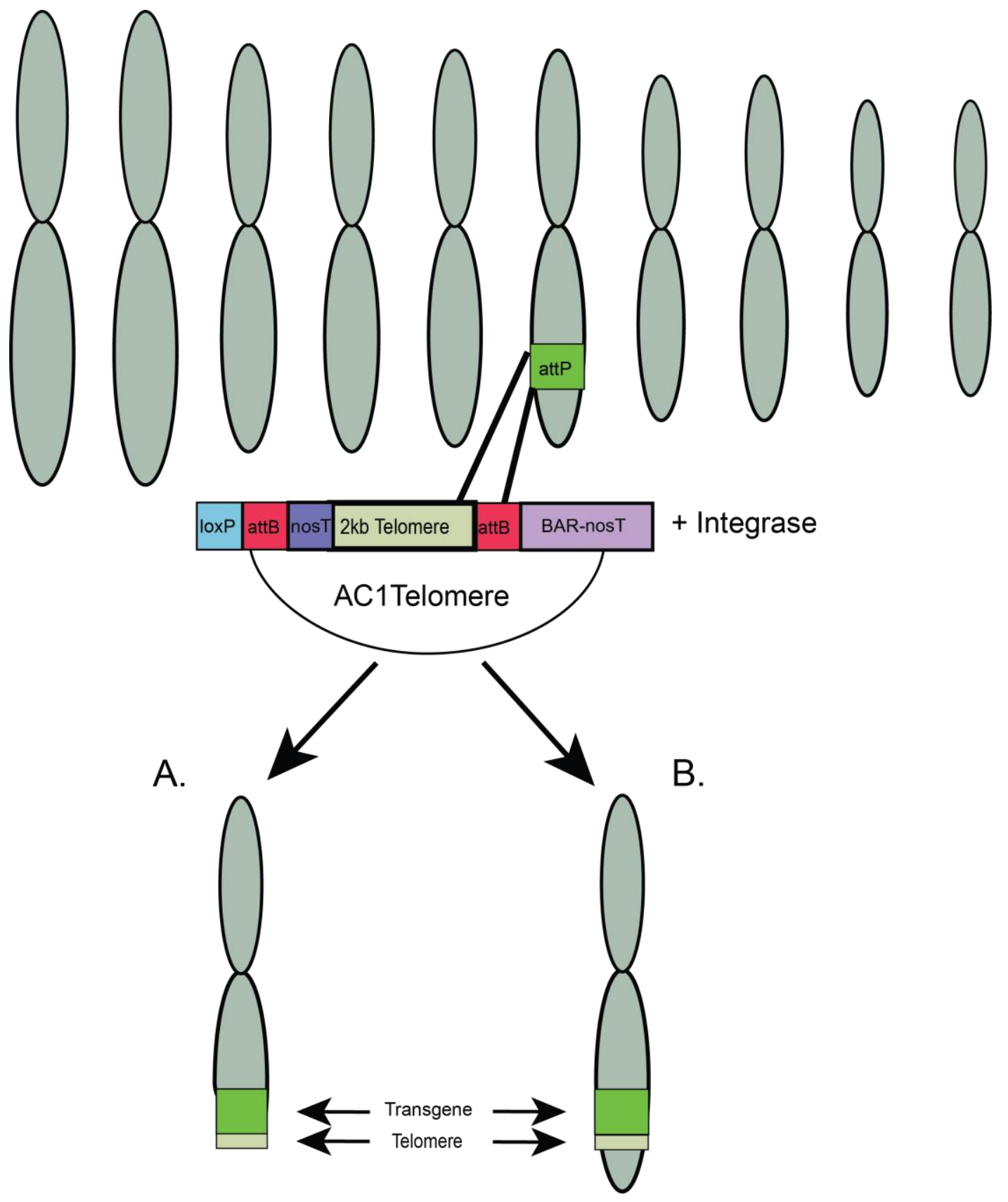

Figure 25: AC1Telomere Possibilities

A: Upon integration of AC1Telo into an attP site located in the genome, if the introduced telomere sequence is recognized by the telomere capping machinery as the new end of the chromosome the result would be a site-specific truncation.

B: If, upon integration of AC1Telomere into an attP site, the introduced telomere sequence is not recognized as the new end of the chromosome by telomere capping machinery, then AC1Telomere will merely by integrated into the genome. 
The B chromosome insertion site of pNT1 11 serves as a good location to test this construct. The ability to truncate the B chromosome at the insertion site and to select for the resulting minichromosome will rely on the pNT1 11 transgene being in the correct orientation. If the orientation of pNT1 11 causes site-specific truncation by AC1Telo to result in the acentric fragment to contain herbicide resistance, then the probable loss of this acentric fragment will result in the inability to select for positive transformants. Correct orientation of pNT1 11 would allow for positive transformants to be selected by BAR after site-specific truncation by AC1Telo, as the acentric fragment would not contain BAR expression in this case. AC1Telo was bombarded in two forms: linear and circular. The linear form was generated by cutting the plasmid with the restriction enzyme BstEII HF from New England Biolabs. The rationale for using the linear form is that truncation may occur at a higher frequency if the strand switching reaction upon site-specific recombination results in the telomere arrays from AC1Telo being the terminal end of the chromosome. 


\section{Supplemental Figures}

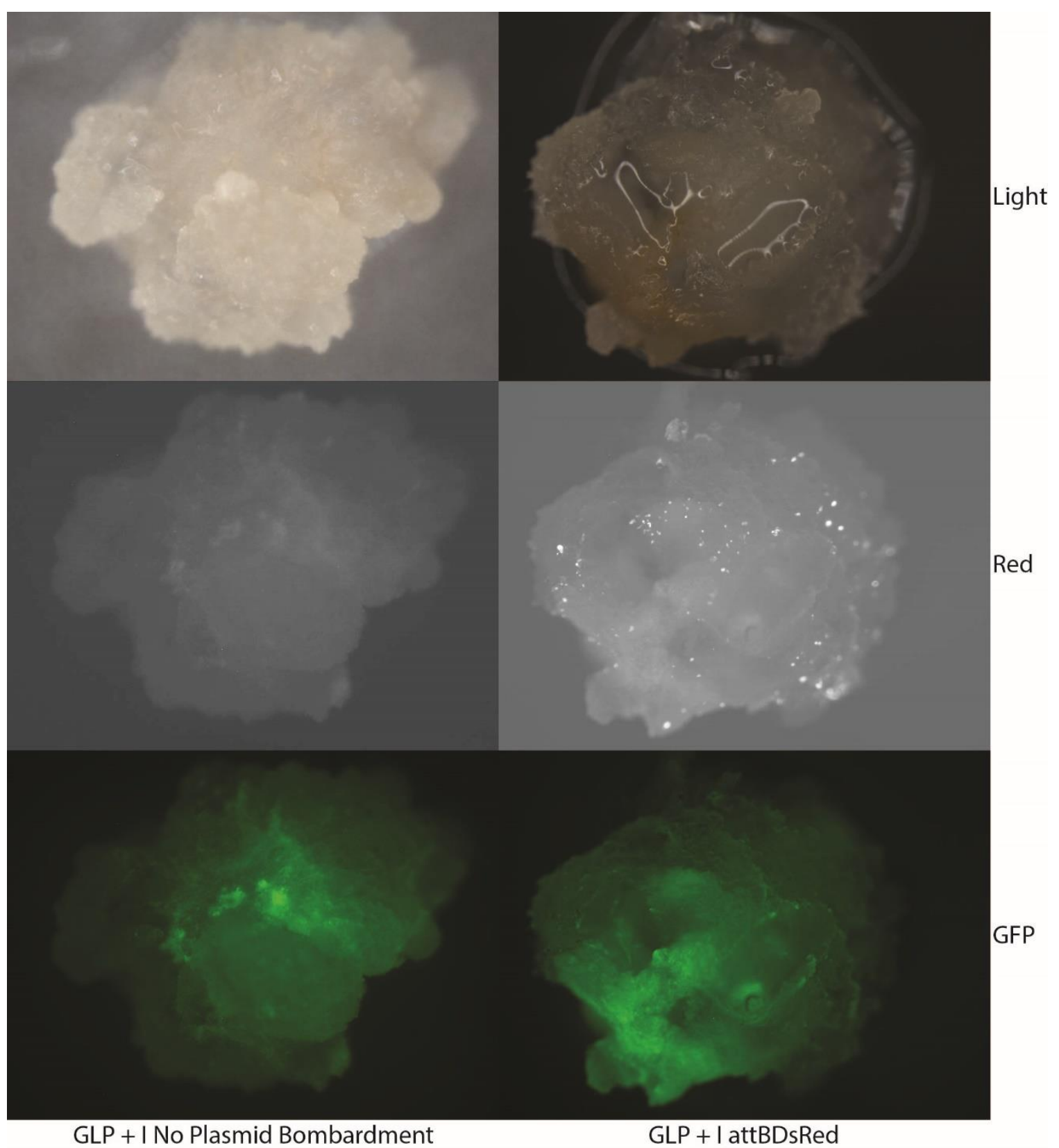

Supplemental Figure 1: Bombardment of attBDsRed into pPZPGLP1+phiC31 Integrase embryos

Fluorescent images of experimental and no plasmid control bombardments. Embryos for bombardment were obtained from ears containing Cre-reduced pPZPGLP1 (Figure 20a) and phiC31 Integrase (Figure 3d). Images on the first column are derived from embryos bombarded by gold particles that were not coated in plasmid DNA. Column two images are derived from embryos that were bombarded by gold particles coated in the plasmid attBDsRed (Figure 20b). Images on the row labeled "Light" are white light images of the embryos. The row labeled "Red" displays embryos under red light fluorescence in grayscale designed to show expression of DsRed. Sections of the experimental column clearly show expression of DsRed while the control column lacks DsRed Expression. The row labeled "GFP" shows embryos under light designed to excite GFP expression, used here as a control to rule out autofluorescence as the source for the areas showing DsRed expression. 
Works Cited

Armstrong, C. L., Green, C. E., and Phillips, R. L. (1991). Development and Availability of Germplasm with High Type-II Culture Formation Response. Maize Genetics Coop Newsletter:92-93.

Benke, K.; Tomkins, B., (2017) Future food-production systems: vertical farming and controlled-environment agriculture, Sustainability: Science, Practice and Policy, 13:1, 13-26, DOI: 10.1080/15487733.2017.1394054.

Bevan, M.W.; Flavell, R.B.; Chilton, M., (1983) A chimaeric antibiotic resistance gene as a selectable marker for plant cell transformation. Nature, 304, 184-187.

Burrack, L.S.; Berman, J. (2012) Neocentromeres and epigenetically inherited features of centromeres. Chromosome Res., 20, 607-619.

Carlson, W. R. (1978). THE B CHROMOSOME OF CORN. Annual Review of Genetics 12(1): 5-5.

Cody, J.P.; Swyers, N.C.; McCaw, M.E.; Graham, N.D.; Zhao, C.; Birchler, J.A. (2015) Minichromosomes: Vectors for Crop Improvement. Agronomy, 5, 309-321.

Comai, L.; Maheshwari, S.; Marimuthu, M. P. A., (2017) Plant centromeres, Current Opinion in Plant Biology, Volume 36, Pages 158-167, ISSN 1369-5266, https://doi.org/10.1016/j.pbi.2017.03.003. 
Dale, E. C. and D. W. Ow (1990). Intra- and intramolecular site-specific recombination in plant cells mediated by bacteriophage $\mathrm{P} 1$ recombinase. Gene 91(1): 79-85.

Fitzgerald, M.S.; McKnight, T.D.; Shippen, D.E. (1996) Characterization and developmental patterns of telomerase expression in plants. Proc. Natl. Acad. Sci. U. S. A., 93, 14422-14427.

Fraley, R.T.; Rogers, S.G.; Horsch, R.B.; Sanders, P.R.; Flick, J.S.; Adams, S.P.; Bittner, M.L.; Brand, L.A.; Fink, C.L.; Fry, J.S., (1983) Expression of bacterial genes in plant cells. Proc. Natl. Acad. Sci. U. S. A., 80, 4803-4807.

Frame B. R., Zhang, H., Cocciolone, S. M., Sidorenko, L. V., Dietrich, C. R., Pegg, S. E., Zhen, S., Schnable, P. S., Wang, K. (2000). Production of Transgenic Maize from Bombarded Type II Callus: Effect of Gold Particle Size and Callus Morphology on Transformation Efficiency. In Vitro Cellular \& Developmental Biology. Plant 36(1): 21-29.

Fu S., Lv Z., Gao Z., Wu H., Pang J., Zhang B., et al. (2013). De novo centromere formation on a chromosome fragment in maize. Proc. Natl. Acad. Sci. U.S.A. 110 6033-6036. 10.1073/pnas.1303944110

Gaeta, R. T., et al. (2012). Synthetic Chromosome Platforms in Plants. Annual Review of Plant Biology 63(1): 307-330.

García-Otín AL, Guillou F. (2006), Mammalian genome targeting using sitespecific recombinases. Front Biosci. 2006 Jan 1;11:1108-36. 
Han F, Lamb, J. C., Birchler, J. A. (2006) High frequency of centromere inactivation resulting in stable dicentric chromosomes of maize. Proc Natl Acad Sci U. S. A.;103(9):3238-3243. doi:10.1073/pnas.0509650103

Henning, K. A., Novotny, E. A., Compton, S. T., Guan, X., Liu, P. P., Ashlock, M. A., (1999). Human artificial chromosomes generated by modification of a yeast artificial chromosome containing both human alpha satellite and single-copy DNA sequences. Proc Natl Acad Sci U. S. A. 96(2): 592-597.

Herrera-Estrella, L.; Depicker, A.; Montagu, V.M.; Schell, J. (1983) Expression of chimaeric genes transferred into plant cells using a Ti-plasmid-derived vector. Nature, 303, 209-213.

Hirschi, K.D. (2009) Nutrient biofortification of food crops. Annu. Rev. Nutr, 29, $401-421$.

Houben A. (2017) B Chromosomes - A Matter of Chromosome Drive. Front Plant Sci. 2017;8:210. doi:10.3389/fpls.2017.00210

Ishida, Y., Hiei, Y., Komari, T., (2007). Agrobacterium-mediated transformation of maize. Nature Protocols 2: 1614.

Kanizay, L.; Dawe, R. (2009) Centromeres: Long intergenic spaces with adaptive features. Funct. Integr. Genomics, 9, 287-292.

Kapusi, E.; Ma, L.; Teo, C.H.; Hensel, G.; Himmelbach, A.; Schubert, I.; Mette, M.; Kumlehn, J.; Houben, A. (2012) Telomere-mediated truncation of barley chromosomes. Chromosoma, 121, 181-190. 
Kato, A.; Lamb, J.C.; Birchler, J.A. (2004) Chromosome painting using repetitive DNA sequences as probes for somatic chromosome identification in maize. Proc. Natl. Acad. Sci. USA, 101, 13554-13559.

Keravala, A., Groth, A. C., Jarrahian, S. (2006) A diversity of serine phage integrases mediate site-specific recombination in mammalian cells. Mol Genet Genomics 276: 135. https://doi.org/10.1007/s00438-006-0129-5

Kilian, A.; Heller, K.; Kleinhofs, A. (1998) Development patterns of telomerase activity in barley and maize. Plant Mol. Biol., 37, 621-628.

Leach, K. A., McSteen, P. C. and Braun, D. M. (2016). Genomic DNA isolation from maize (Zea mays) leaves using a simple, high-throughput protocol. Current Protocols in Plant Biology 1: 15- 27. doi: 10.1002/cppb.20000

Leonard, A.C.; Mechali, M. (2013) DNA replication origins. Cold Spring Harb. Perspect. Biol., 5, doi:10.1101/cshperspect.a010116.

Li R., Han Z., Hou L., Kaur G., Yin Q., Ow D.W. (2016) Method for Biolistic SiteSpecific Integration in Plants Catalyzed by Bxb1 Integrase. In: Murata M. (eds) Chromosome and Genomic Engineering in Plants. Methods in Molecular Biology, vol 1469. Humana Press, New York, NY

Lowe, K., Wu, E., Wang, N., Hoerster, G., Hastings, C., Cho, M., Scelonge, C., Lenderts, B., Chamberlin, M., Cushatt, J., Wang, L., Ryan, L., Khan, T., ChowYiu, J., Hua, W., Yu, M., Banh, J., Bao, Z., Brink, K., Igo, E., Rudrappa, B., Shamseer, P. M., Bruce, W., Newman, L., Bo Shen, Zheng, P., Bidney, D., Falco, C., Register, J., Zhao, Z., Xu, D., Jones, J., Gordon-Kamm, W., (2016). 
Morphogenic Regulators Baby boom and Wuschel Improve Monocot Transformation. The Plant Cell 28(9): 1998-2015.

Lyznik, L. A., Mitchell, J. C., Hirayama, L., Hodges, T. K., (1993) Activity of yeast FLP recombinase in maize and rice protoplasts, Nucleic Acids Research, Volume 21, Issue 4, 25 February, Pages 969-975, https://doi.org/10.1093/nar/21.4.969 Makarov, V.L.; Hirose, Y.; Langmore, J. (2001) Long g tails at both ends of human chromosomes suggest a $\mathrm{C}$ strand degradation mechanism for telomere shortening. Cell, 88, 657-666

Matzke, A.J.; Matzke, M.A. (1988) Position effects and epigenetic silencing of plant transgenes. Curr. Opin. Plant Biol., 1, 142-148.

McCaw M, Graham N, Cody J, Swyers N, Zhao C, Birchler J (2016a) Fluorescence in situ hybridization to maize (Zea mays) chromosomes. Current Protocols in Plant Biology 1:530-545.

McCaw M, Swyers N, Graham N, Cody J, Zhao C, Birchler J (2016b) Preparation of Chromosomes from Zea mays. Current Protocols in Plant Biology 1:501-509. Moon H.S., Abercrombie L.L., Eda S., Blanvillain R., Thomson J.G., Ow D.W. and Stewart C.N. Jr. (2011) Transgene excision in pollen using a codon optimized serine resolvase $\mathrm{CinH}-\mathrm{RS} 2$ site-specific recombination system. Plant Mol. Biol. 5, 621-631.

Murai, N.; Kemp, J.D.; Sutton, D.W.; Murray, M.G.; Slightom, J.L.; Merlo, D.; Reichert, N.A.; Sengupta-Gopalan, C.; Stock, C.A.; Barker, R.F. (1983) 
Phaseolin gene from bean is expressed after transfer to sunflower via tumorinducing plasmid vectors. Science, 222, 476-482.

Murray, A. W. and J. W. Szostak (1983). Construction of artificial chromosomes in yeast. Nature 305(5931): 189-193.

Nakagawa, Y., Machida, C., Machida, Y. (2001) A system to induce the deletion of genomic sequences using R/RS site-specific recombination and the Ac transposon in transgenic rice plants Theor Appl Genet 102: 1136. https://doi.org/10.1007/s001220100580

Nelson, A. D., et al. (2011). Parameters Affecting Telomere-Mediated Chromosomal Truncation in Arabidopsis. The Plant Cell 23(6): 2263-2272.

Ow, D. W. (2011), Recombinase-mediated Gene Stacking as a Transformation Operating System. Journal of Integrative Plant Biology, 53: 512-519. doi:10.1111/j.1744-7909.2011.01061.

Roman, H. (1947) Mitotic nondisjunction in the case of interchanges involving the B-type chromosome in maize. Genetics, 32, 391-409.

Sambrook, J., Fritsch, E. F., and Maniatis, T. (1989). Molecular Cloning: A Laboratory Manual. Cold Spring Harbor laboratory press.

Singer, K., Shiboleth, Y. M., Li, J., Tzfira, T., (2012). Formation of Complex Extrachromosomal Structures in Agrobacterium tumefaciens-Infected Plants. Plant Physiology 160(1): 511-522. 
Swyers, N. C., Cody, J. P., McCaw, M. E., Graham, N. D., Zhao, C., and Birchler, J. A. (2016). Telomere-mediated chromosomal truncation for generating engineered minichromosomes in maize. Curr. Protoc. Plant Biol. 1: 488- 500. doi: 10.1002/cppb.20031

Teo, C.H.; Ma, L.; Kapusi, E.; Hensel, G.; Kumlehn, J.; Schubert, I.; Houben, A.; Mette, M.F. (2011) Induction of telomere-mediated chromosomal truncation and stability of truncated chromosomes in Arabidopsis thaliana. Plant J., 68, 28-39 Thomson J. G., Chan R., Thilmony R., Yau Y. Y., Ow D. W. (2010) PhiC31 recombination system demonstrates heritable germinal transmission of sitespecific excision from the Arabidopsis genome. BMC Biotechnol. 2010;10:17. Published Feb 23. doi:10.1186/1472-6750-10-17.

Thorpe, H. M. and M. C. M. Smith (1998). In vitro site-specific integration of bacteriophage DNA catalyzed by a recombinase of the resolvase/invertase family. Proc Natl Acad Sci U. S. A. 95(10): 5505-5510.

Vaucheret, H. , Béclin, C. , Elmayan, T. , Feuerbach, F. , Godon, C. , Morel, J. , Mourrain, P. , Palauqui, J. and Vernhettes, S. (1998), Transgene-induced gene silencing in plants. The Plant Journal, 16: 651 -659. doi:10.1046/j.1365313x.1998.00337.

Vega, J., Yu, W., Kennon, A., Chen, X. and Zhang, Z. (2008). Improvement of Agrobacterium-mediated transformation in Hi-II maize (Zea mays) using standard binary vectors. Plant Cell Reports. 27, 2 (2008), 297-305 
Xu, C. , Cheng, Z. and Yu, W. (2012), Construction of rice mini-chromosomes by telomere-mediated chromosomal truncation. The Plant Journal, 70: 1070-1079. doi:10.1111/j.1365-313X.2012.04916.x

Xu, Z. and Dooner, H. K., (2006). The Maize aberrant pollen transmission 1 Gene Is a SABRE/KIP Homolog Required for Pollen Tube Growth. Genetics 172(2): 1251-1261.

Yamada, Y., Maeda, M., Alshahni, M., Monod, M., Staib, P. and Yamada, T. (2014). Flippase (FLP) recombinase-mediated marker recycling in the dermatophyte Arthroderma vanbreuseghemii. Microbiology. 160, Pt_10 (2014), 2122-2135.

Yan, X. , Li, C. , Yang, J. , Wang, L. , Jiang, C. and Wei, W. (2017), Induction of telomere-mediated chromosomal truncation and behavior of truncated chromosomes in Brassica napus. Plant J, 91: 700-713. doi:10.1111/tpj.13598.

Yu, W.; Han, F.; Gao, Z.; Vega, J.M.; Birchler, J.A. (2007) Construction and behavior of engineered minichromosomes in maize. Proc. Natl. Acad. Sci. U. S. A., 104, 8924-8929.

Yu, W., Lamb, J. C., Han, F, Birchler, J. A. (2006) Telomere-mediated chromosomal truncation in maize. Proc Natl Acad Sci U. S. A.;103(46):1733117336. doi:10.1073/pnas.0605750103.

Yuan, J., Shi, Q., Guo, X., Liu, Y., Su, H., Guo, X., Lv, Z., Han, F., (2017) Sitespecific transfer of chromosomal segments and genes in wheat engineered 
chromosomes, Journal of Genetics and Genomics, doi:

10.1016/j.jgg.2017.08.005.

Zhong, C. X. ; Marshall, J. B. ; Topp, C.; Mroczek, R.; Kato, A.; Nagaki, K.;

Birchler, J.A.; Jiang, J.; Dawe, R.K. (2002) Centromeric retroelements and satellites interact with maize kinetochore protein CENH3. Plant Cell, 14, 28252836.

Zhu, C.; Naqvi, S.; Gomez-Galera, S.; Pelacho, A.M.; Capell, T.; Christou, P. (2007) Transgenic strategies for the nutritional enhancement of plants. Trends Plant Sci., 12, 548-555. 


\section{Appendix 1: Amendment Construct Maps}

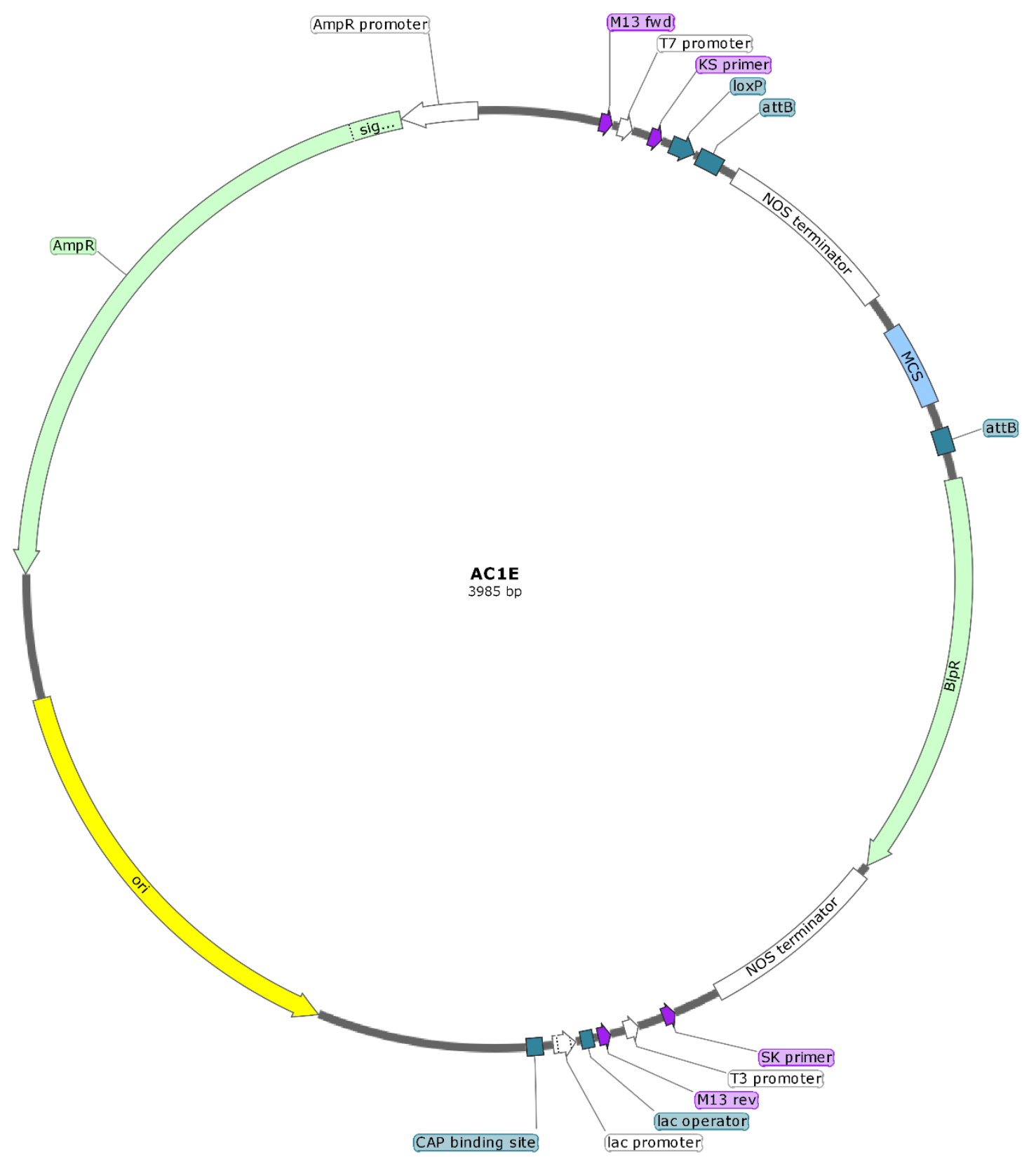




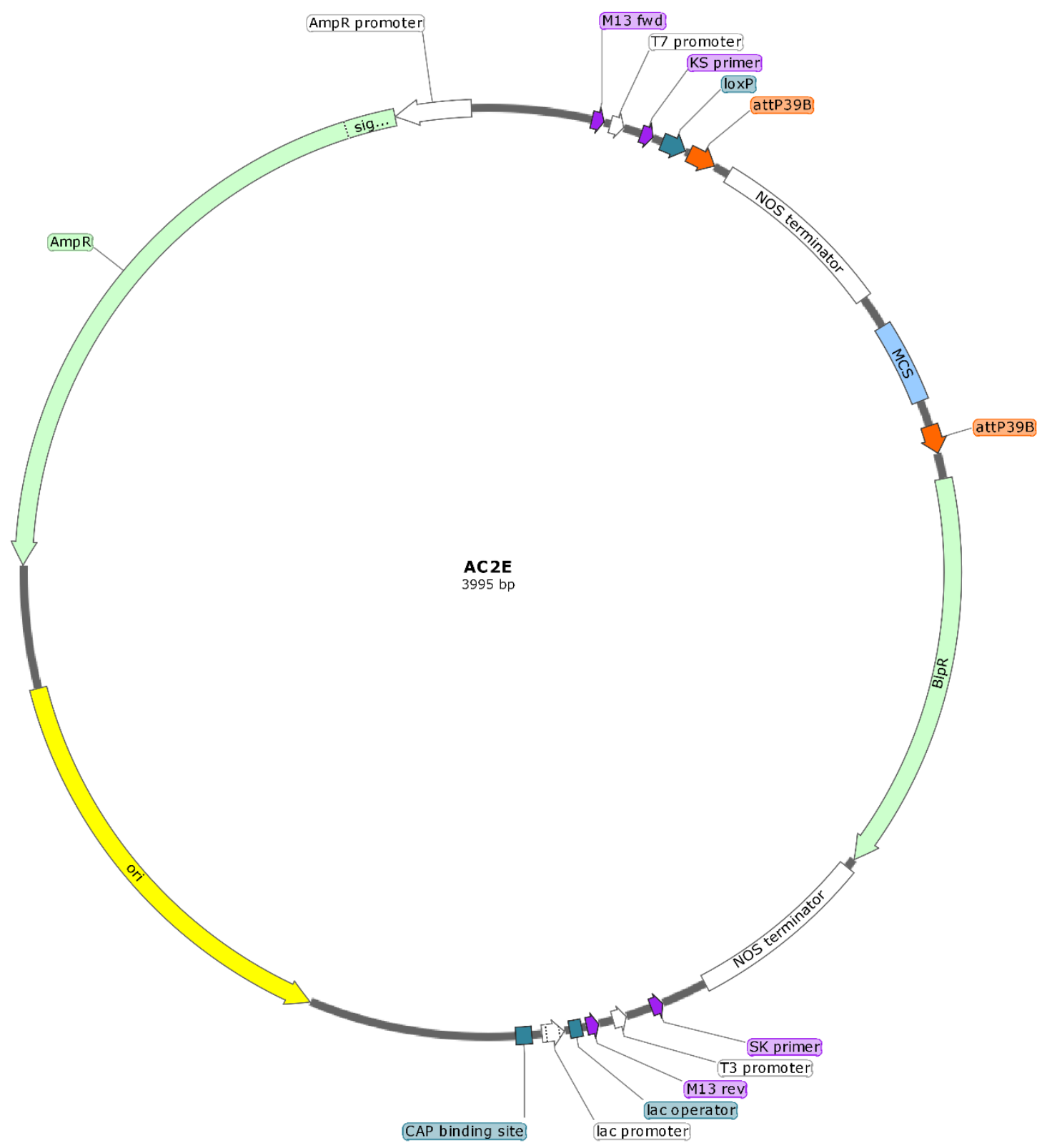




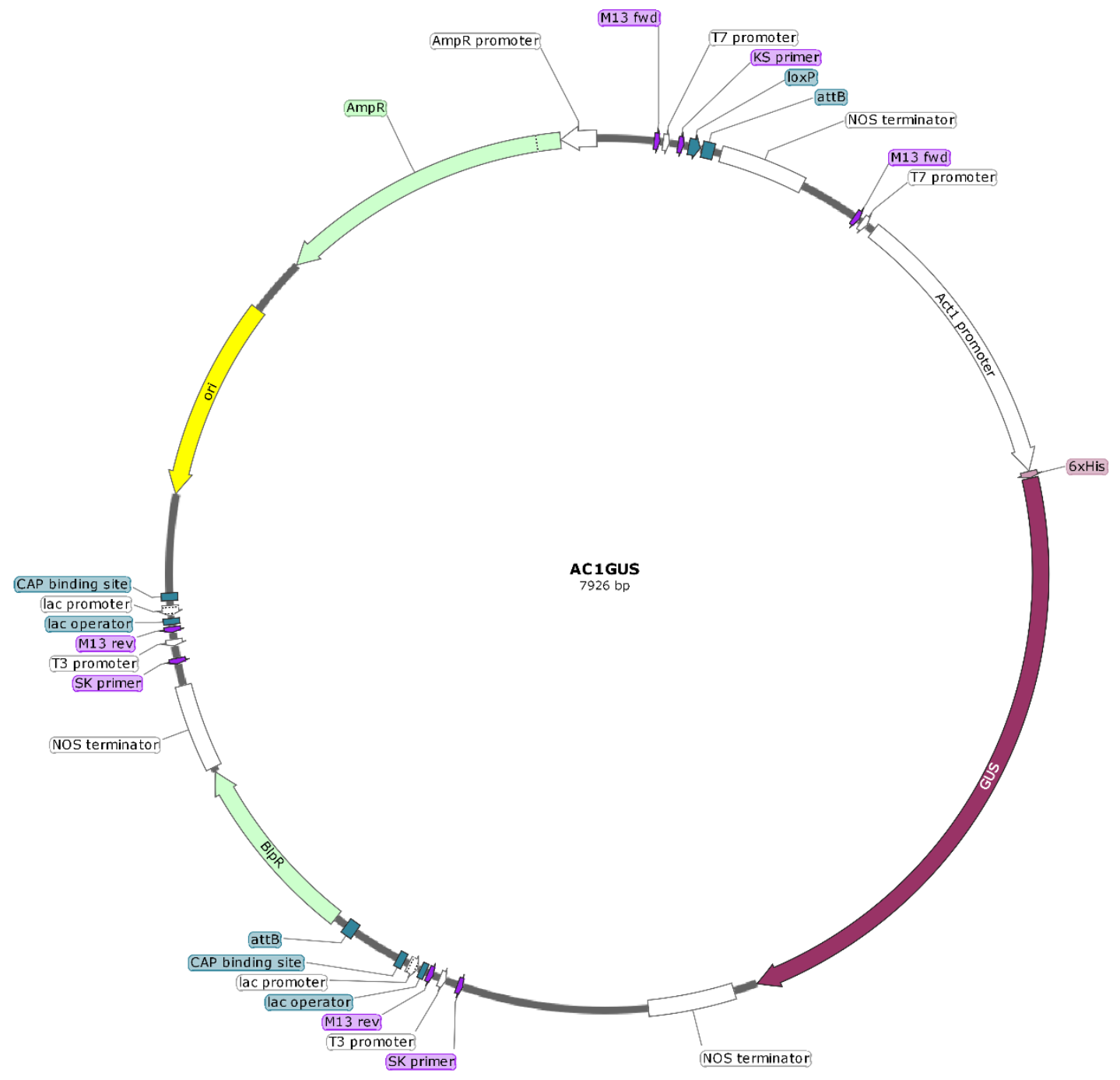




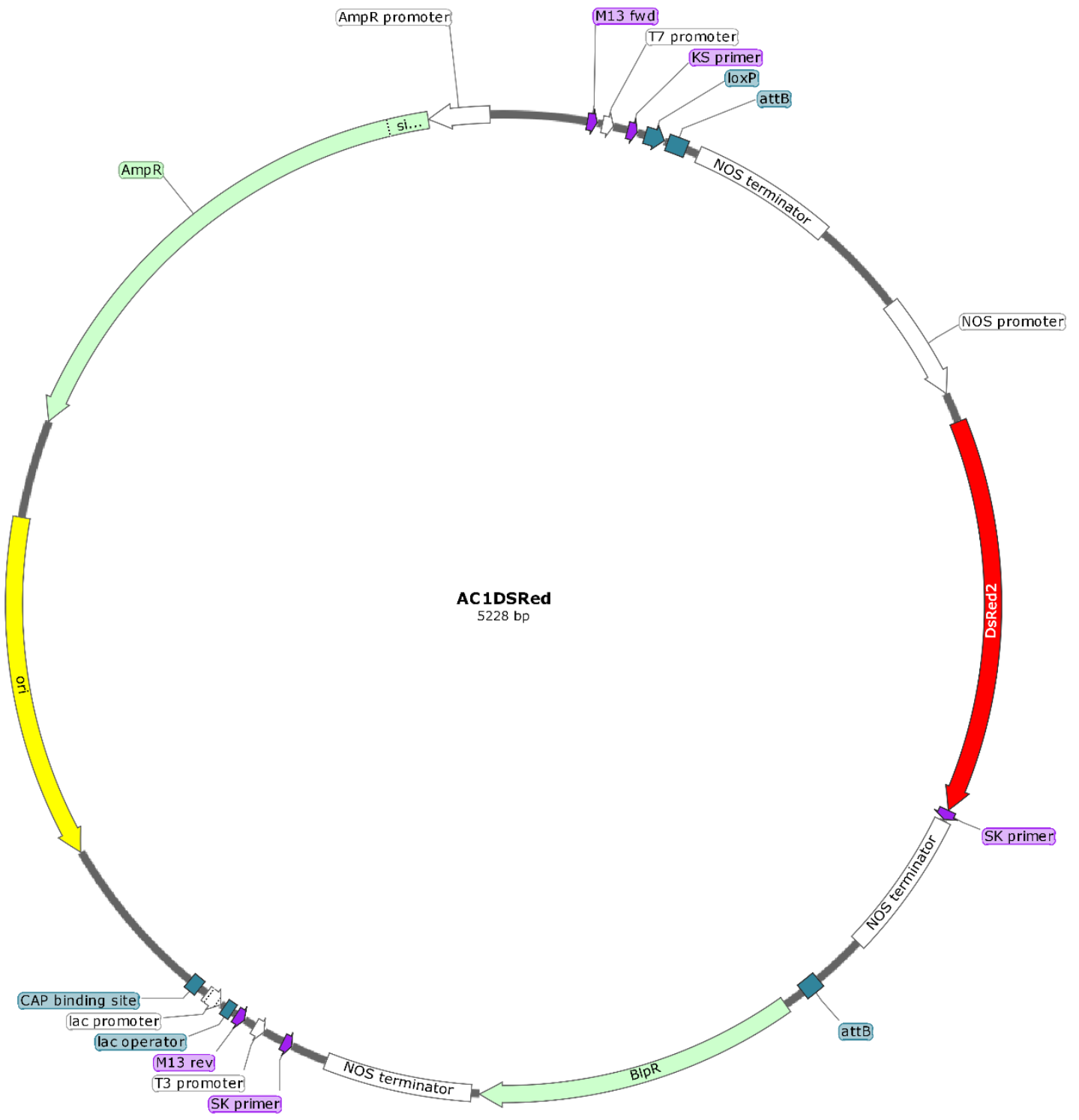




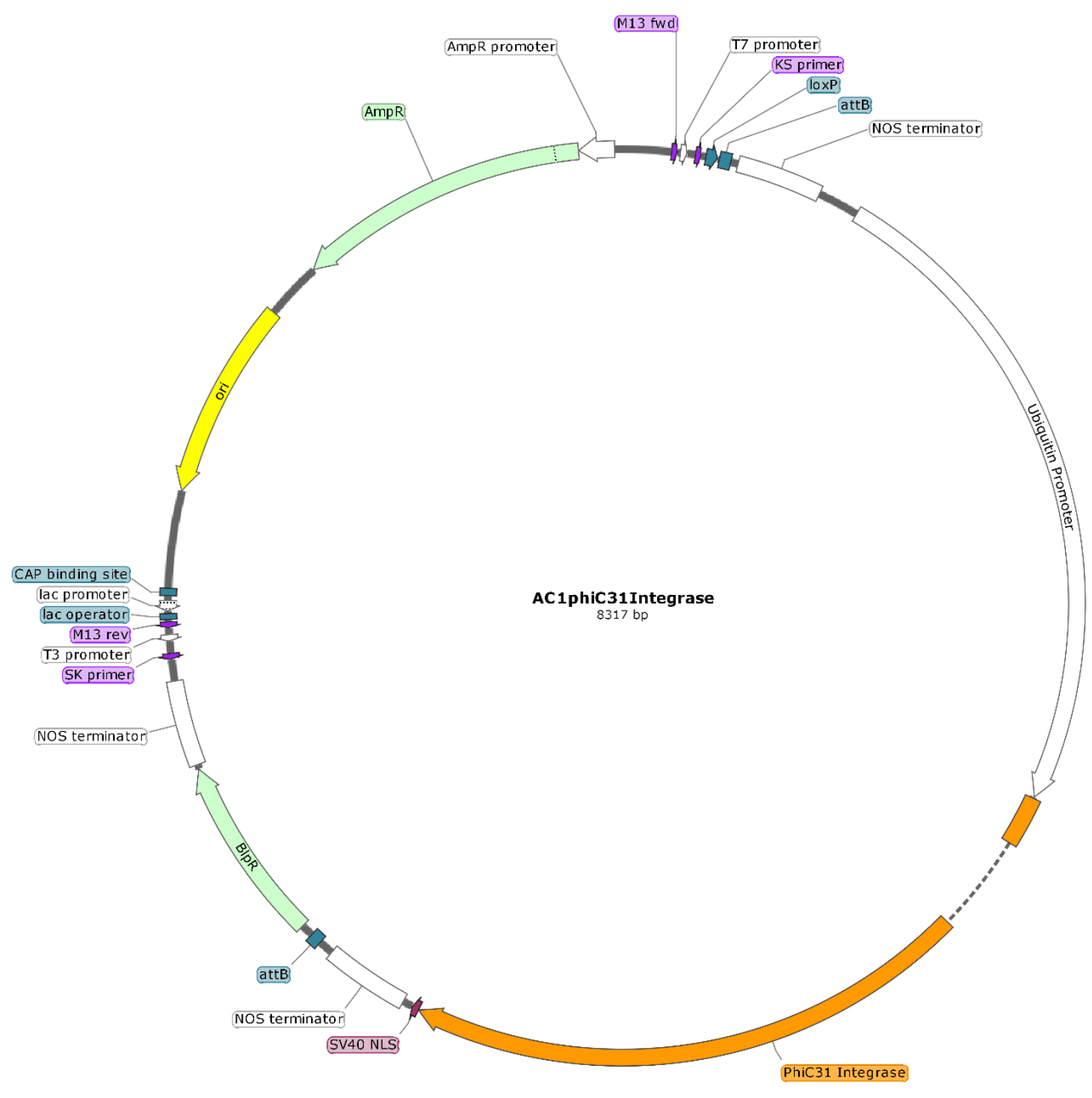




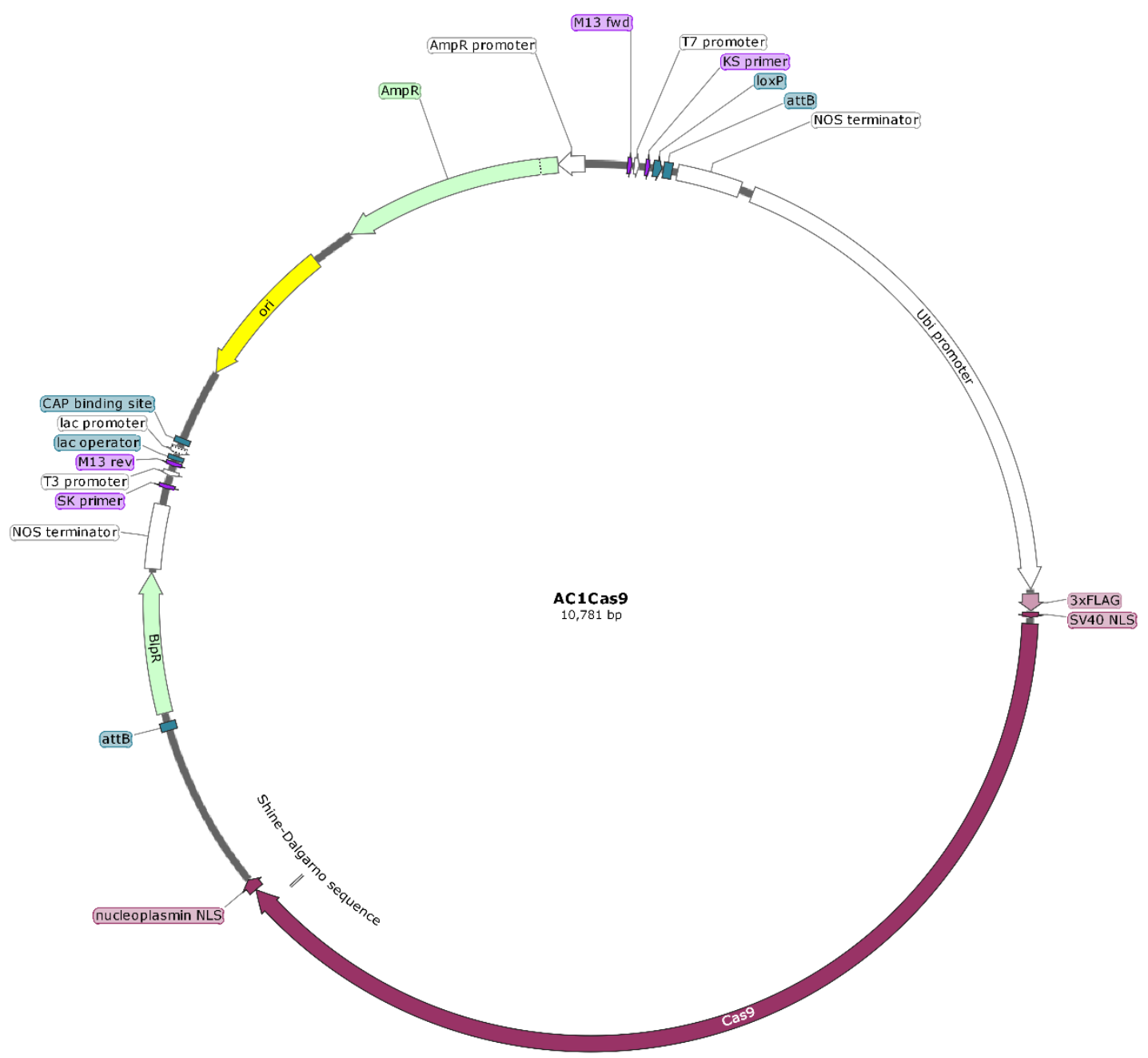




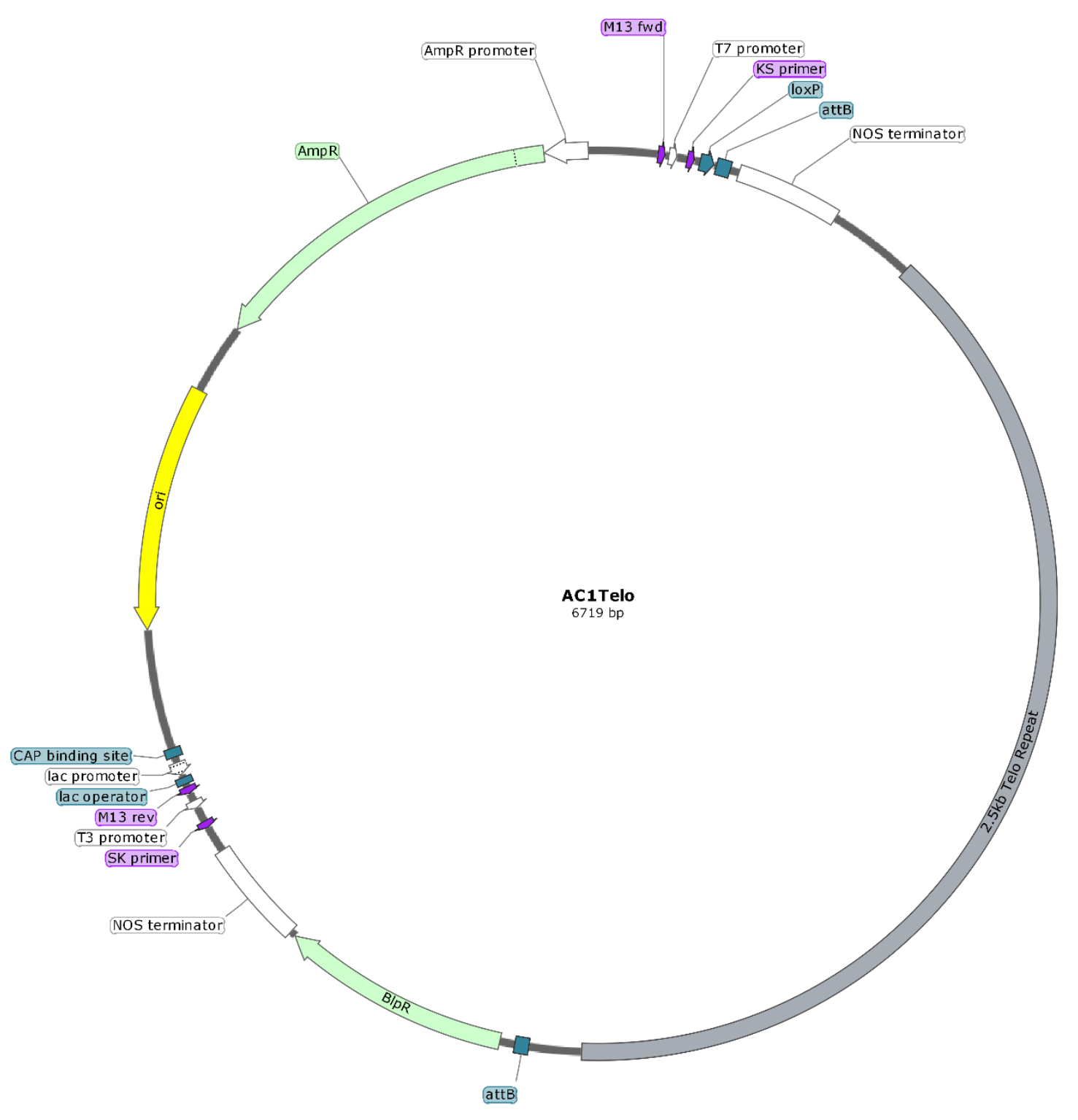




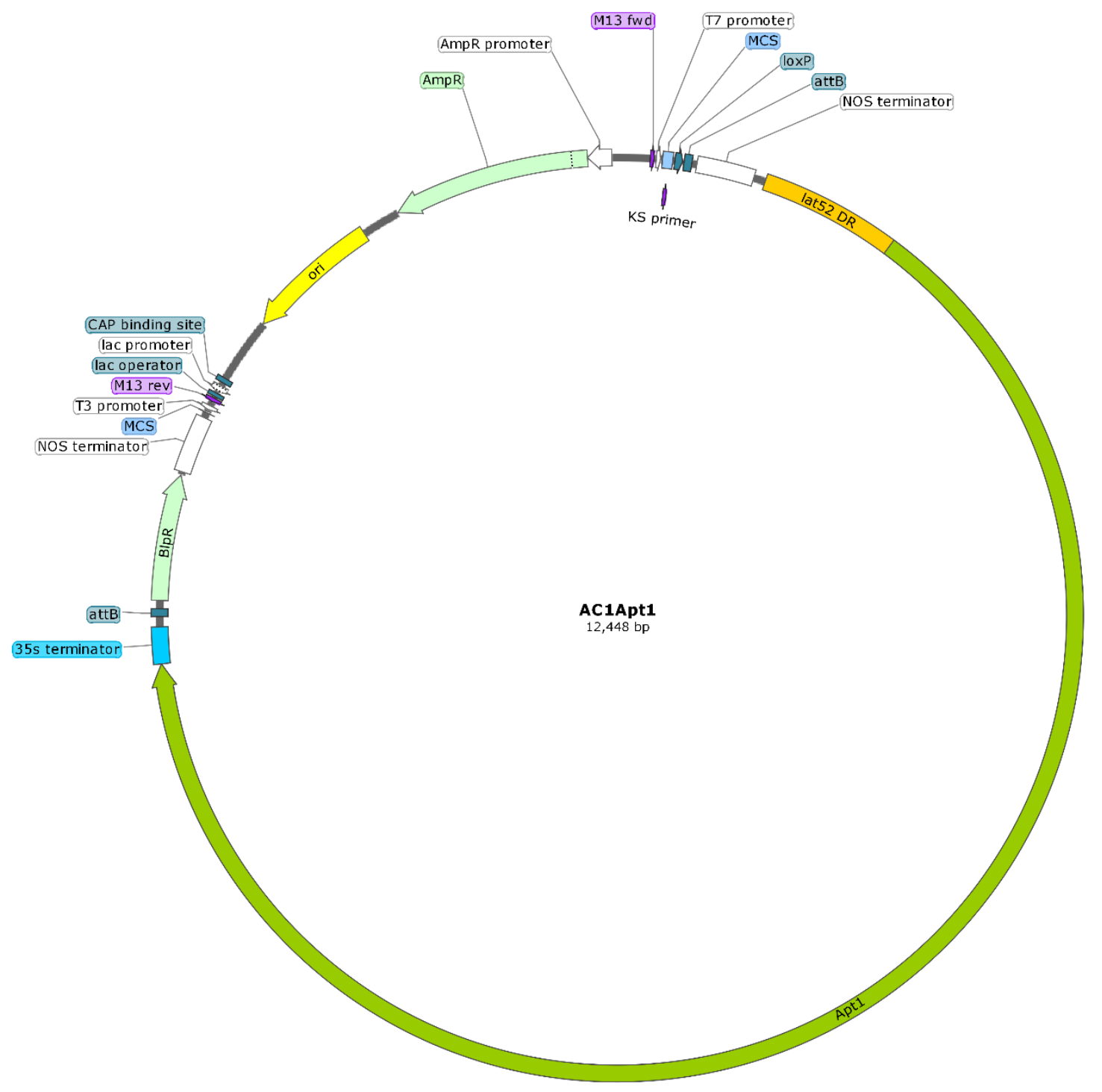




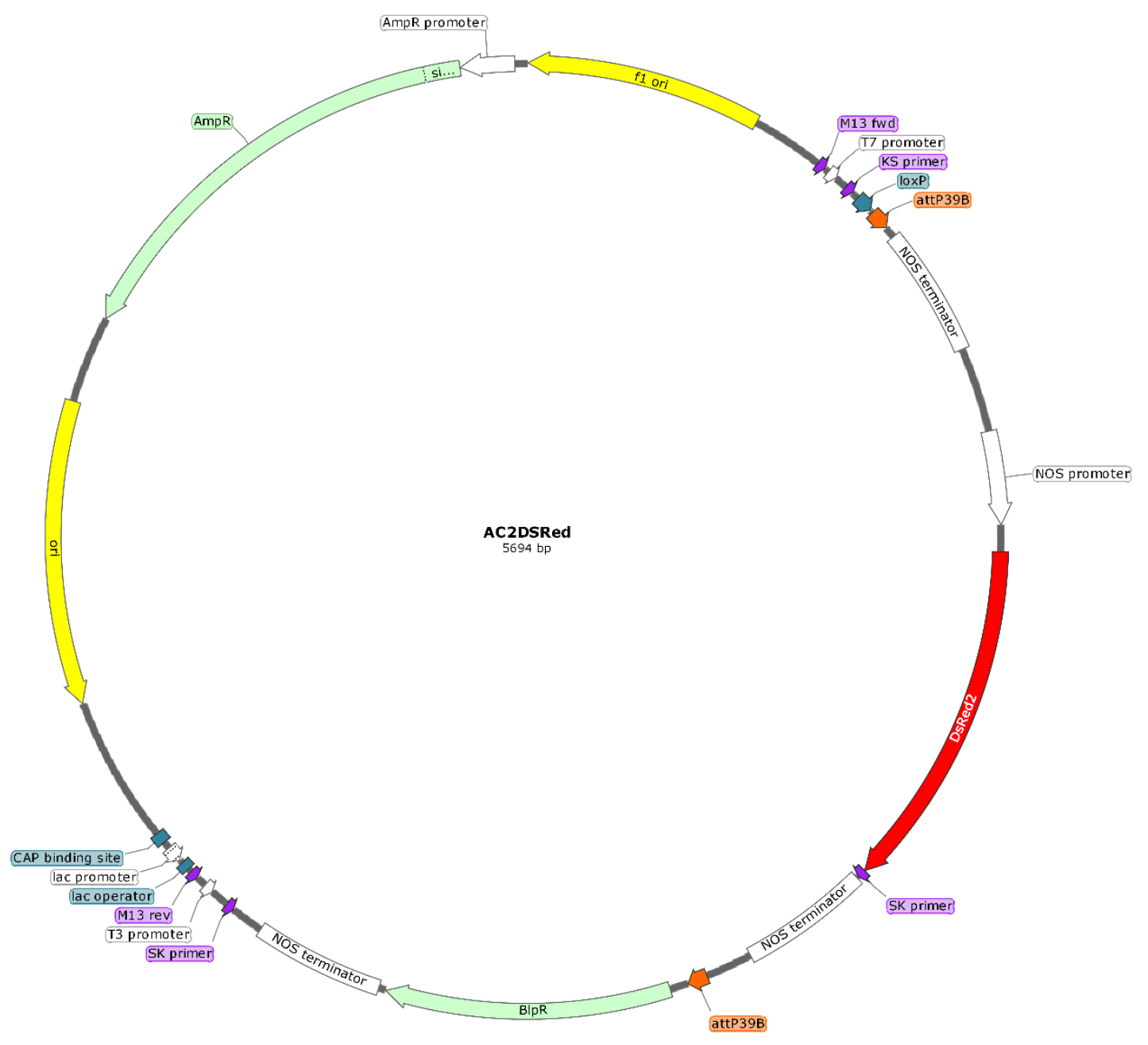




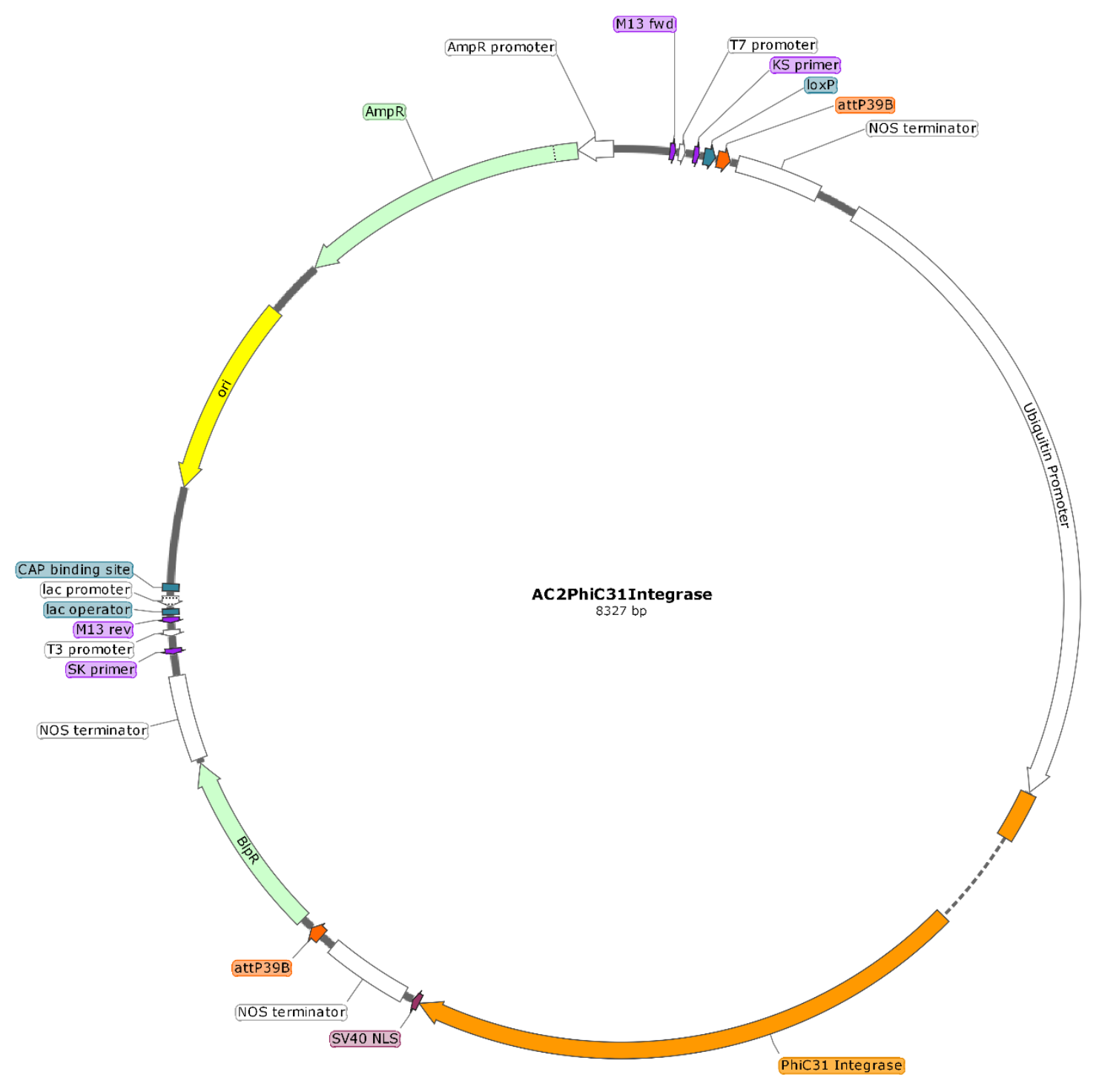




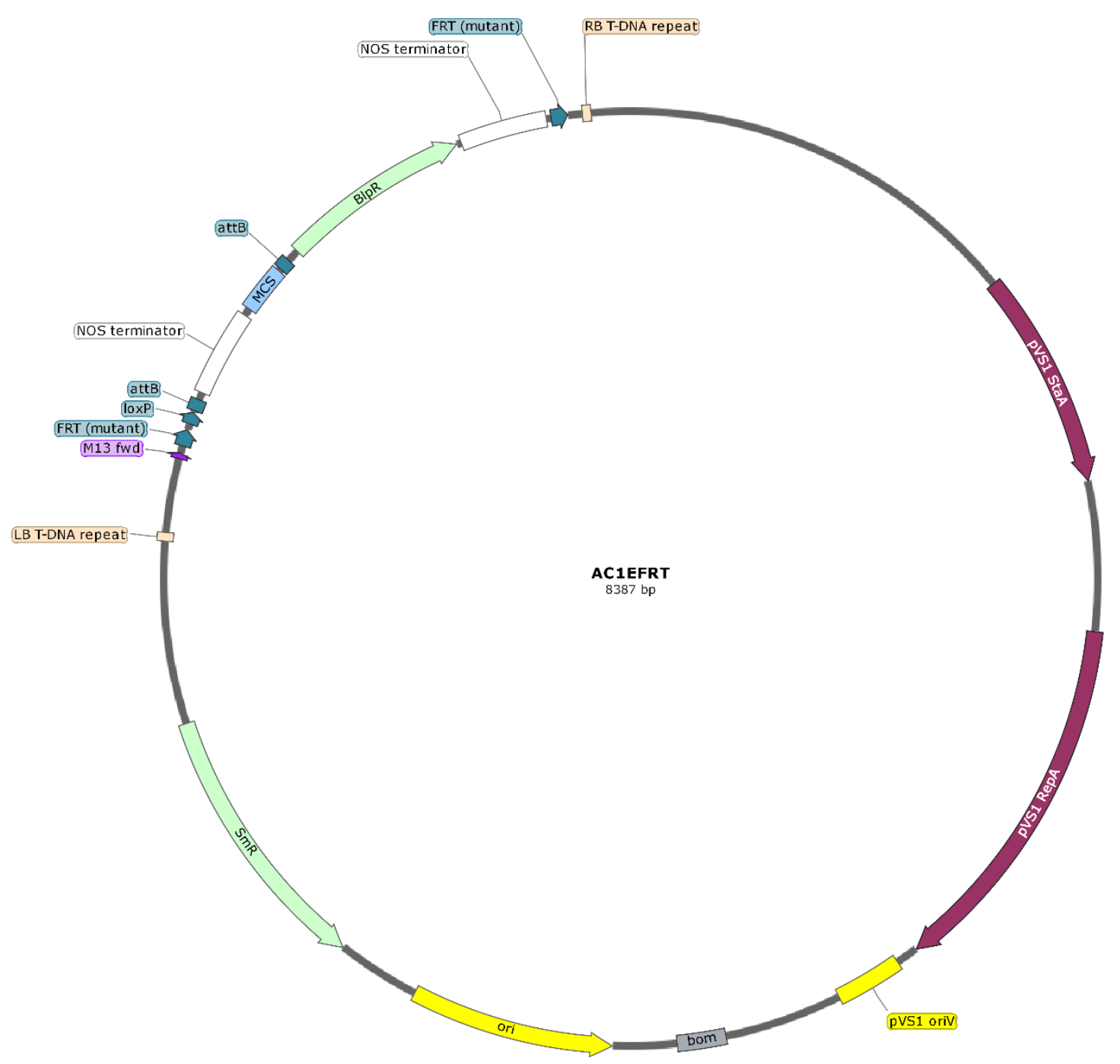

96 


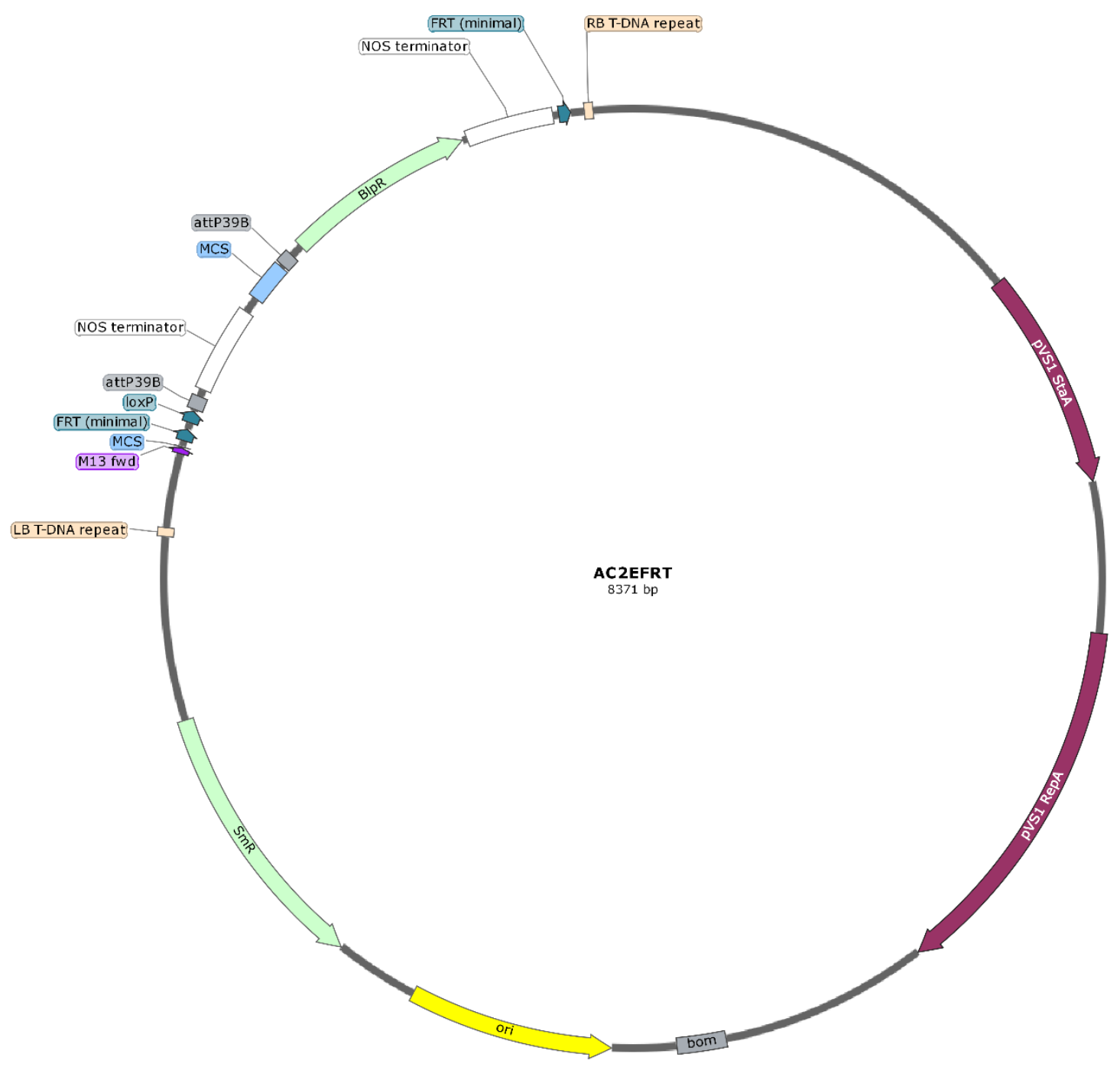

97 


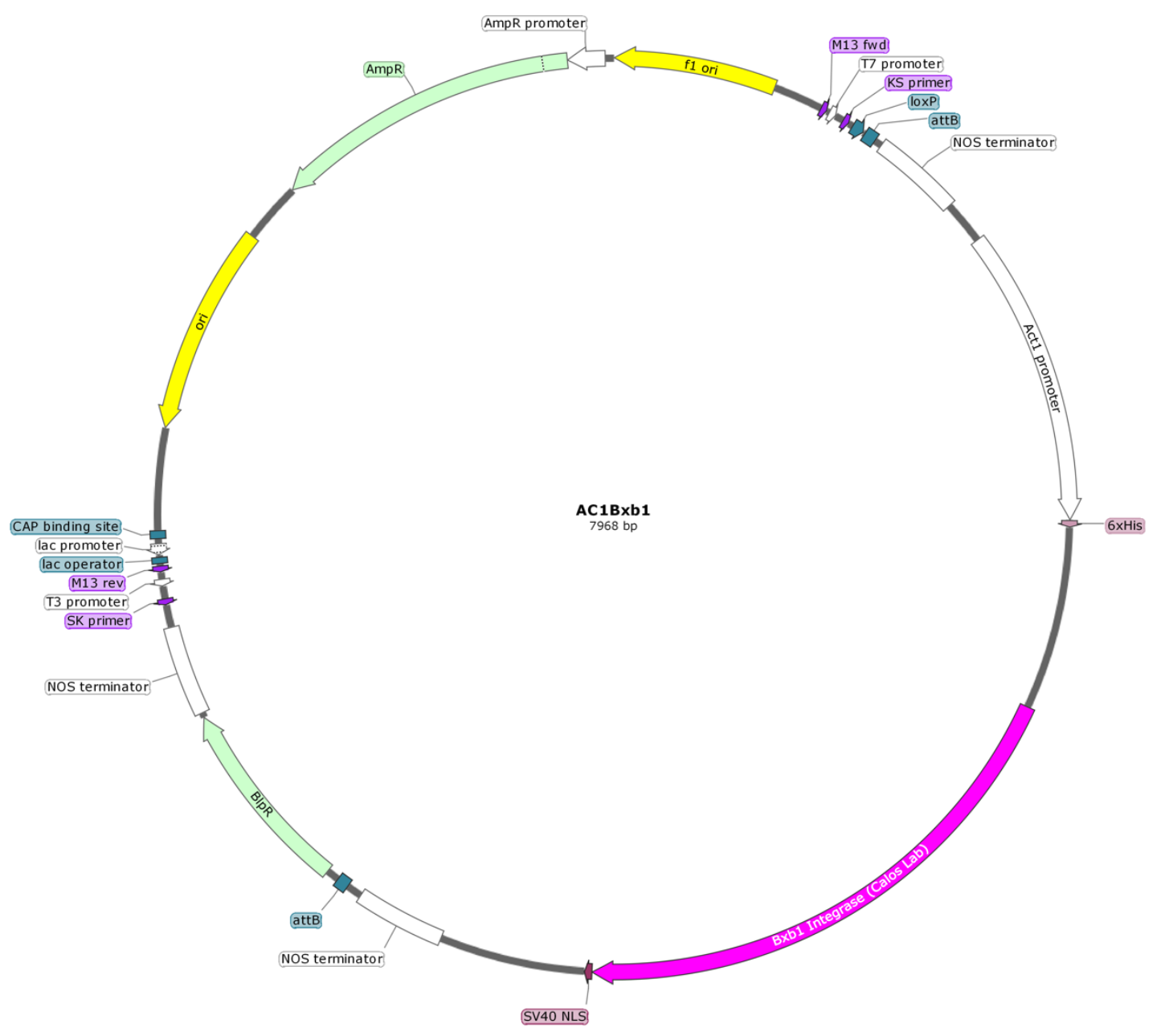




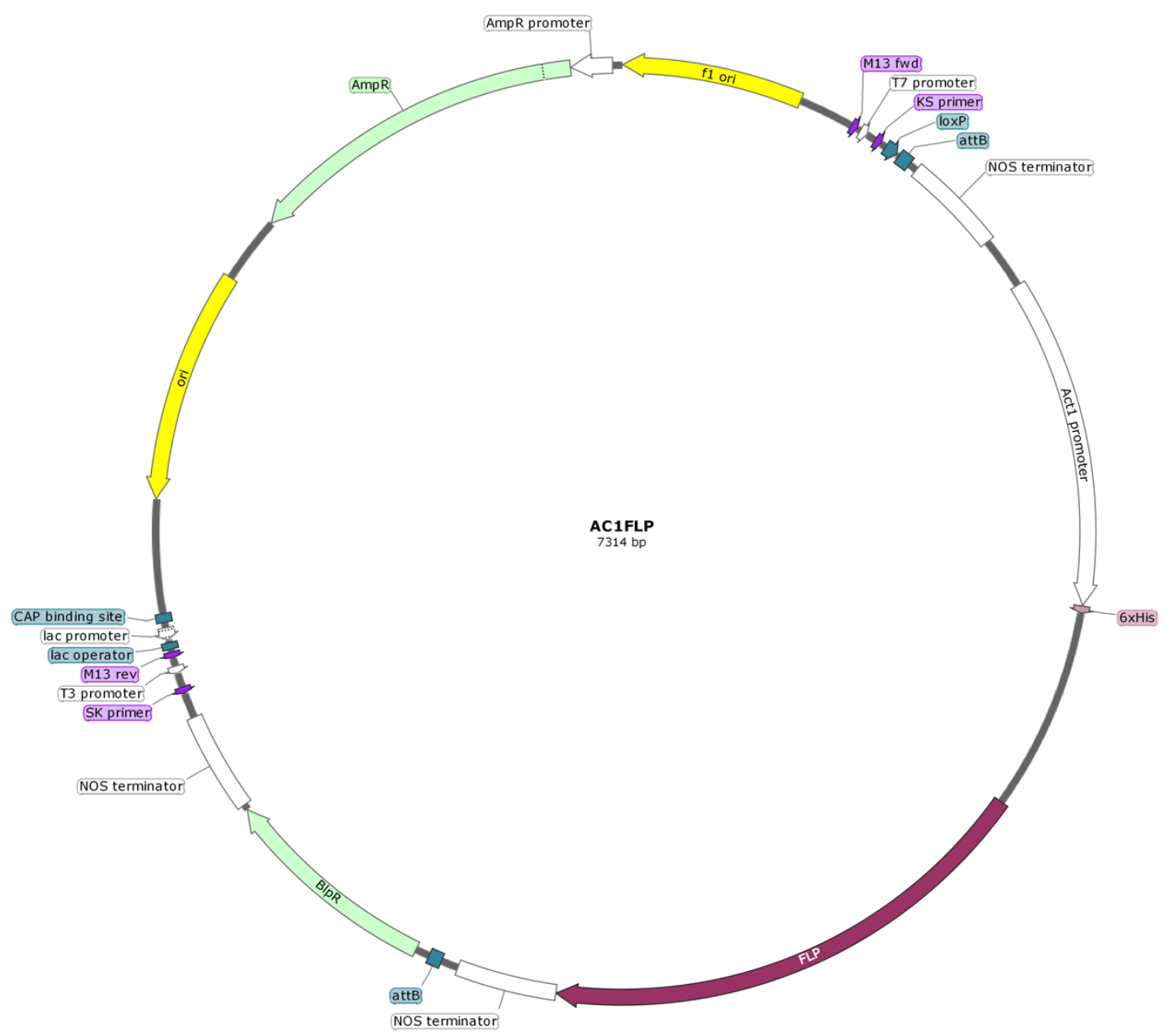




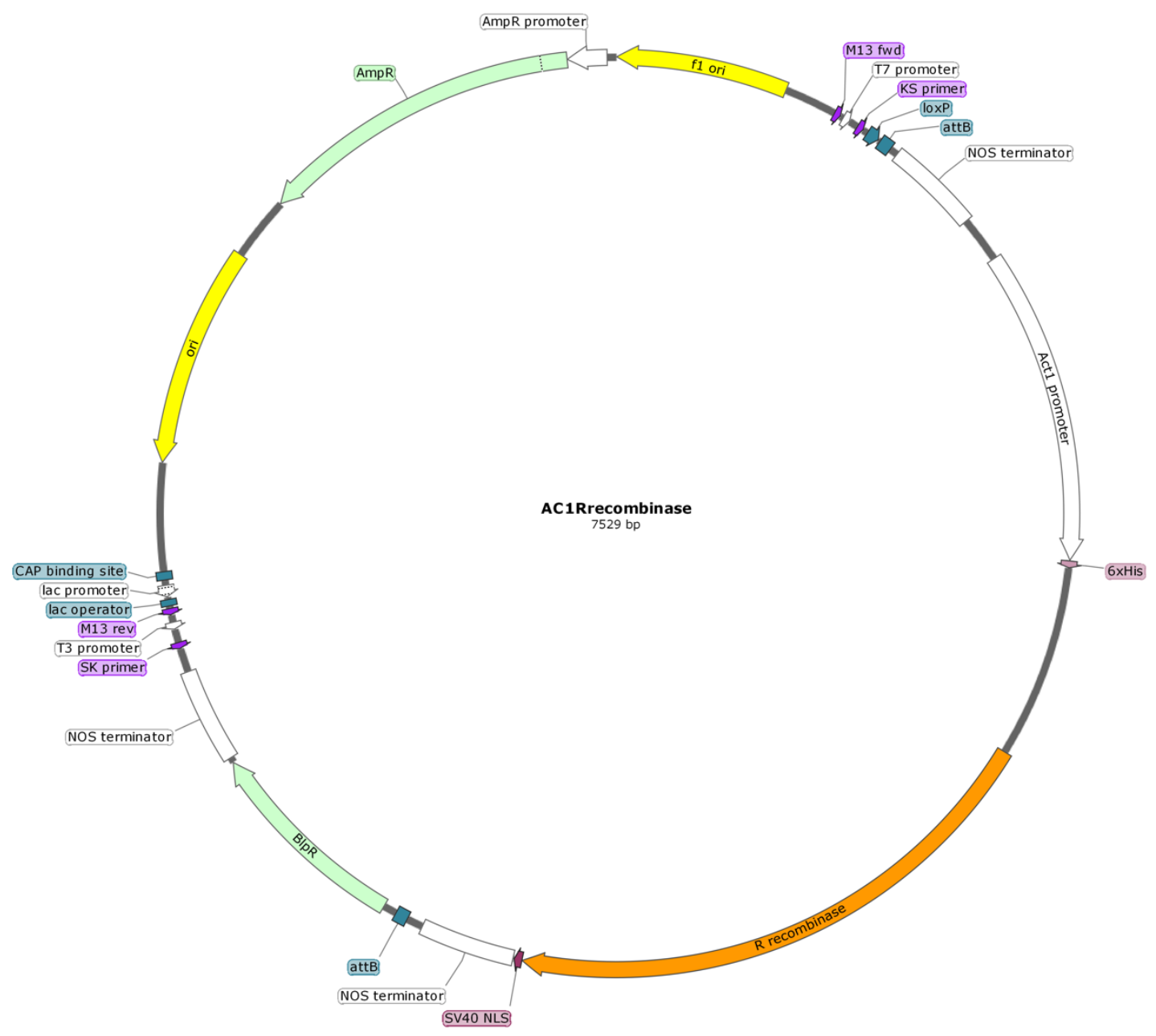




\section{Appendix 2: Telomere-Mediated Chromosomal Truncation for Generating Engineered Minichromosomes in Maize}

Note: The information in this chapter was published under the title:

Swyers, N. C., Cody, J. P., McCaw, M. E., Graham, N. D., Zhao, C., and Birchler, J. A. (2016). Telomere-mediated chromosomal truncation for generating engineered minichromosomes in maize. Curr. Protoc. Plant Biol. 1: 488- 500. doi: 10.1002/cppb.20031

\section{Summary}

Minichromosomes have been generated in maize using telomere-mediated truncation. Telomere DNA, because of its repetitive nature, can be difficult to manipulate. The protocols in this unit describe two methods for generating the telomere DNA required for the initiation of telomere-mediated truncation. The resulting DNA can then be used with truncation cassettes for introduction into maize via transformation.

\section{INTRODUCTION}

Artificial minichromosomes are small chromosomes that exist autonomously from the native chromosomal set.Minichromosomes contain all the necessary components needed for maintenance and stability in a cellular environment: a centromere, telomeres, and an origin of replication (Murray and Szostak, 1983). In addition, a selectable marker is also included for positive identification of successful artificial minichromosome construction (Gaeta et al., 2012). Since the initial development of an artificial minichromosome in yeast (Murray and Szostak, 1983), production has been demonstrated in a number of organisms including 
bacterial, fungal, mammalian, and plant systems (Murray and Szostak, 1983;

Farr et al., 1991; Shizuya et al., 1992; Heller et al., 1996; Yu et al., 2006).

Minichromosome construction is predominantly carried out in one of two ways:

the bottom-up or top-down method. The bottom-up method is an assemblage of sequence components necessary for chromosomal stability, maintenance, and autonomy. While this strategy is successful in a number of organisms, including budding yeast (Murray and Szostak, 1983) and mammals (Harrington et al., 1997), the bottom-up method has yet to be demonstrated in plants despite recent claims of success (Carlson et al., 2007; Ananiev et al., 2009), which have been critiqued (Gaeta et al., 2012). Indeed, the epigenetic nature of plant centromeres (Birchler and Han, 2009) is a major complication that might preclude the bottom-up approach.

Alternatively, the top-down method, or minichromosome creation via telomeremediated truncation, has been successful in a number of plant systems, including maize, rice, barley, and Arabidopsis (Yu et al., 2006; Nelson et al., 2011; Teo et al., 2011; Kapusi et al., 2012; Xu et al., 2012). In this strategy, constructs containing genes of interest and telomere arrays are delivered into a host genome via Agrobacterium transformation or particle bombardment, resulting in cleavage and recovery of pre-existing chromosomes upon integration. The resulting chromosome has an endogenous centromere that has never left the cell, but the arms are removed, and transgenes are added in the same process. Most components incorporated into a truncation construct require the use of common molecular cloning techniques, such as Gibson assembly (Gibson 
et al., 2009) or restriction digest followed by ligation. However, the repetitive nature of telomere arrays complicates the utilization of these strategies.

Therefore, more advanced cloning methods are required. Here, two cloning strategies for telomere incorporation are described in detail. These include in-gel ligation and telomere repeat concatenation via PCR followed by ligation.

\section{IN-GEL LIGATION OF TELOMERE DNA INTO TARGET PLASMID}

Working with telomere sequences is complicated by their instability. As a result, standard cloning techniques will often fail when attempting to introduce telomere repeats into plasmids. In order to combat this issue, telomere sequences are extracted and ligated within an agarose gel to bypass techniques that might shear them. Despite this, it is still necessary to screen resulting clones for the size of the resulting telomere inserts.

While the length of telomere sequence required for telomere truncation is not known, it is suggested that the largest size possible is chosen to ensure successful minichromosome creation.

\section{Materials}

LB plates (see recipe) containing $100 \mathrm{mg} / \mathrm{ml}$ spectinomycin (e.g, SigmaAldrich, cat. no. S4014)

Plasmid pWY82 (e.g., Addgene, cat. no. 65721) 
$2 \mathrm{X}$ yeast extract tryptone (YT) medium (see recipe)

QIAprep Spin Miniprep Kit (e.g., Qiagen, cat. no. 27104)

Nuclease-free water

Restriction endonucleases compatible with pWY82 and target plasmid

6X DNA Gel Loading Dye (e.g., Thermo Fisher Scientific, cat. no. R0611)

GeneRuler 1 kb DNA Ladder (e.g., Thermo Fisher Scientific, cat. no.

SM0311)

Agarose (e.g., Sigma-Aldrich, cat. no. A9539)

Ethidium bromide (e.g., Sigma-Aldrich, cat. no. E7637)

Target plasmid for transformation

Low-melting-point agarose (e.g., Invitrogen, cat. no. 16520-050)

1X TAE (see recipe)

Antarctic Phosphatase (e.g., New England BioLabs, cat. no. M0289S)

T4 DNA Ligase and Ligase Reaction Buffer (e.g., New England BioLabs, cat. no.

M0202S)

ElectroMax Stbl4 cells (e.g., Thermo Fisher Scientific, cat. no. 11635-018) SOC medium (e.g., Thermo Fisher Scientific, cat. no. 15544-034)

LB plates (see recipe) containing appropriate antibiotics for transformation vector

Radiolabeled nucleotide: (TTTAGGG) ${ }^{10}$

Agrobacterium

Sterile applicator stick or inoculating loop 
Variable temperature incubator

Variable temperature laboratory shaker

250-ml baffled culture flasks

NanoDrop spectrophotometer

1.7-ml microcentrifuge tubes

Vacuum concentrator

UV transilluminator

Scalpel

$70^{\circ} \mathrm{C}$ and $37^{\circ} \mathrm{C}$ water bath

15-ml culture tubes

Nitrocellulose membrane

Additional reagents and equipment for gel electrophoresis (Green and

Sambrook, 2012), electroporation (Potter and Heller, 2010), colony hybridization

(Sambrook et al., 1989), colony PCR (Woodman, 2008), DNA sequencing

(Shendure et al., 2011), and Southern hybridization (Hoopes, 2012)

\section{Prepare the cells and extract DNA}

1. Prepare LB plates containing $100 \mathrm{mg} / \mathrm{ml}$ spectinomycin.

2. Streak bacteria containing plasmid pWY82 onto LB/spectinomycin plates.

This plasmid possesses $\sim 2.6 \mathrm{~kb}$ of Arabidopsis thaliana telomere repeats and is available from Addgene (plasmid \#65721). Telomere sequences are highly 
conserved across the plant kingdom, and this telomere DNA sequence has worked for telomere-mediated truncation in maize, rice, barley, and Arabidopsis.

3. Incubate at $30^{\circ} \mathrm{C}$ for $48 \mathrm{hr}$.

4. Pick a single colony, and swirl into $3 \mathrm{ml}$ of $2 X \mathrm{YT}$ medium with $100 \mathrm{mg} / \mathrm{ml}$ spectinomycin.

5. Shake culture at $250 \mathrm{rpm}$ for 24 to $48 \mathrm{hr}$ at $30^{\circ} \mathrm{C}$.

Starter culture is grown until culture is cloudy to ensure growth. pWY82 is maintained in Stbl2 cells. Thus, it is slow growing.

6. In a baffled culture flask, add $500 \mu \mathrm{l}$ starter culture to $125 \mathrm{ml}$ of $2 \mathrm{X}$ YT medium. A baffled flask is not required but will result in higher yield.

7. Shake at $250 \mathrm{rpm}$ for $48 \mathrm{hr}$, until the culture reaches an OD600 2.

8. Extract culture using the QIAprep Spin Miniprep Kit. Extract $4 \mathrm{ml}$ of culture per column, and elute with $50 \mu \mathrm{l}$ of $50^{\circ} \mathrm{C}$ nuclease-free water. This step will require $\sim 30$ columns. While there are kits that allow extraction of larger culture volumes, we have found that the miniprep method is most efficient. Pre-warm nucleasefree water to $50^{\circ} \mathrm{C}$ for elution and allow to set on column for at least 3 min before eluting. Yield can be increased by passing the eluate through the same 
column a second time.

9. Pool all miniprep elutions into a single 1.7-ml microcentrifuge tube.

\section{Check the DNA integrity}

10. Reduce the volume in a vacuum concentrator until the concentration is $\sim 1$ $\mu \mathrm{g} / \mu \mathrm{l}$ when measured with a NanoDrop spectrophotometer.

11. Digest $1 \mu \mathrm{g}$ plasmid with each restriction endonuclease to be used individually, following the manufacturer's instructions.

12. Add 6x loading dye after digestion is complete. 


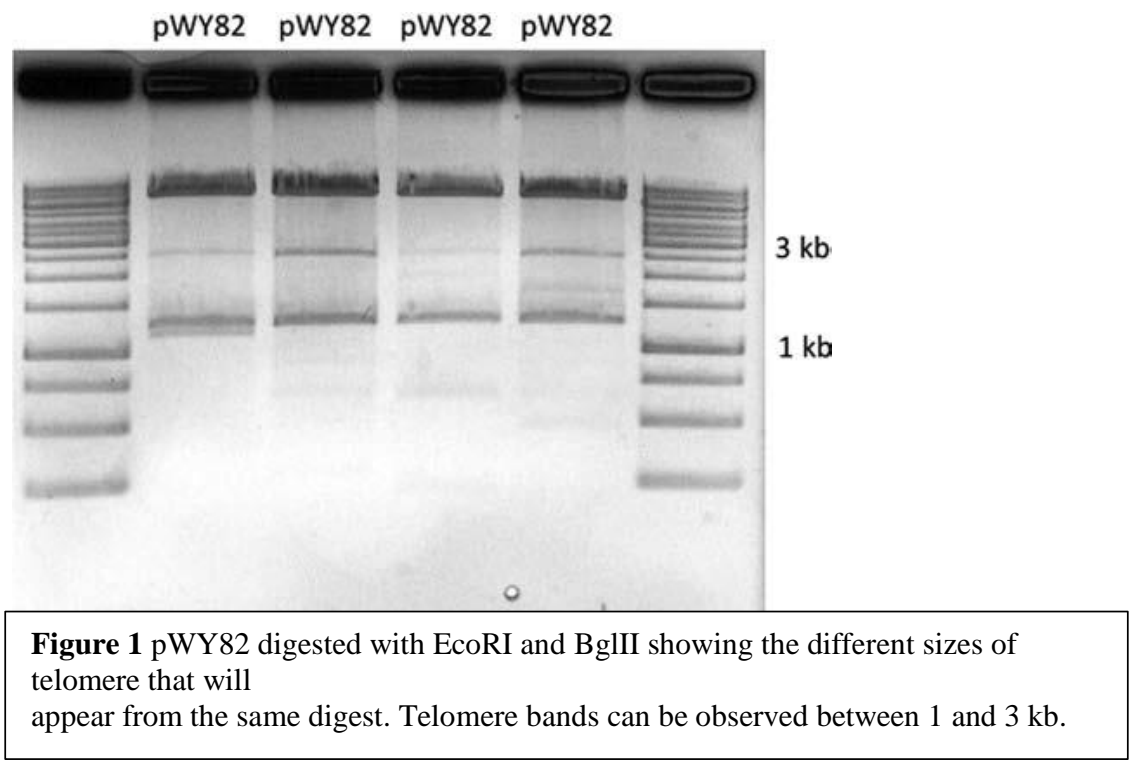


13. Load digest and GeneRuler $1 \mathrm{~kb}$ DNA Ladder into $1 \%(\mathrm{w} / \mathrm{v})$ agarose gel and run until dye approaches the bottom of the gel.

14. Stain with $0.5 \mu \mathrm{g} / \mathrm{ml}$ ethidium bromide and visualize under UV light.

15. Check the integrity of pWY82 and efficiency of restriction endonucleases by examining gel. Telomere repeats are unstable and may form secondary structures when run in an agarose gel. As a result, multiple bands will often appear after gel electrophoresis in addition to the full $2.6 \mathrm{~kb}$ band (Fig. 1). Digest pWY82 and target plasmid and perform electrophoresis

16. Digest $10 \mu \mathrm{g}$ pWY82 and $5 \mu \mathrm{g}$ target plasmid with at least $10 \mathrm{U}$ restriction endonuclease in a total volume of $50 \mu \mathrm{l}$ for $1 \mathrm{hr}$. Digestions of each plasmid should be carried out in separate tubes. The target plasmid should have the gene of interest cloned into it prior to in-gel ligation. This plasmid should be binary if used with Agrobacterium transformation and possess compatible restriction endonuclease cut sites near the right border for the introduction of telomere from pWY82.

17. While the digest is ongoing pour a $1 \%(\mathrm{w} / \mathrm{v})$ low-melting-point agarose gel with 1X TAE. When pouring gel, make sure to use a comb with wells large enough to hold the entire restriction digest and loading dye. 
18. Allow the gel to solidify for at least $30 \mathrm{~min}$ in a $4^{\circ} \mathrm{C}$ cold room. Low-meltingpoint agarose gels are fragile and should be handled with care.

19. After restriction digest is complete, treat target plasmid with $5 \cup$ Antarctic Phosphatase for $15 \min$ at $37^{\circ} \mathrm{C}$.

20. Add $15 \mu \mathrm{l}$ of $6 \mathrm{X}$ loading dye to each reaction and mix by pipetting.

21. Fill electrophoresis chamber with $1 \mathrm{X} T \mathrm{TAE}$, and carefully insert low-meltingpoint gel. Caution should be taken as gel is extremely fragile. 


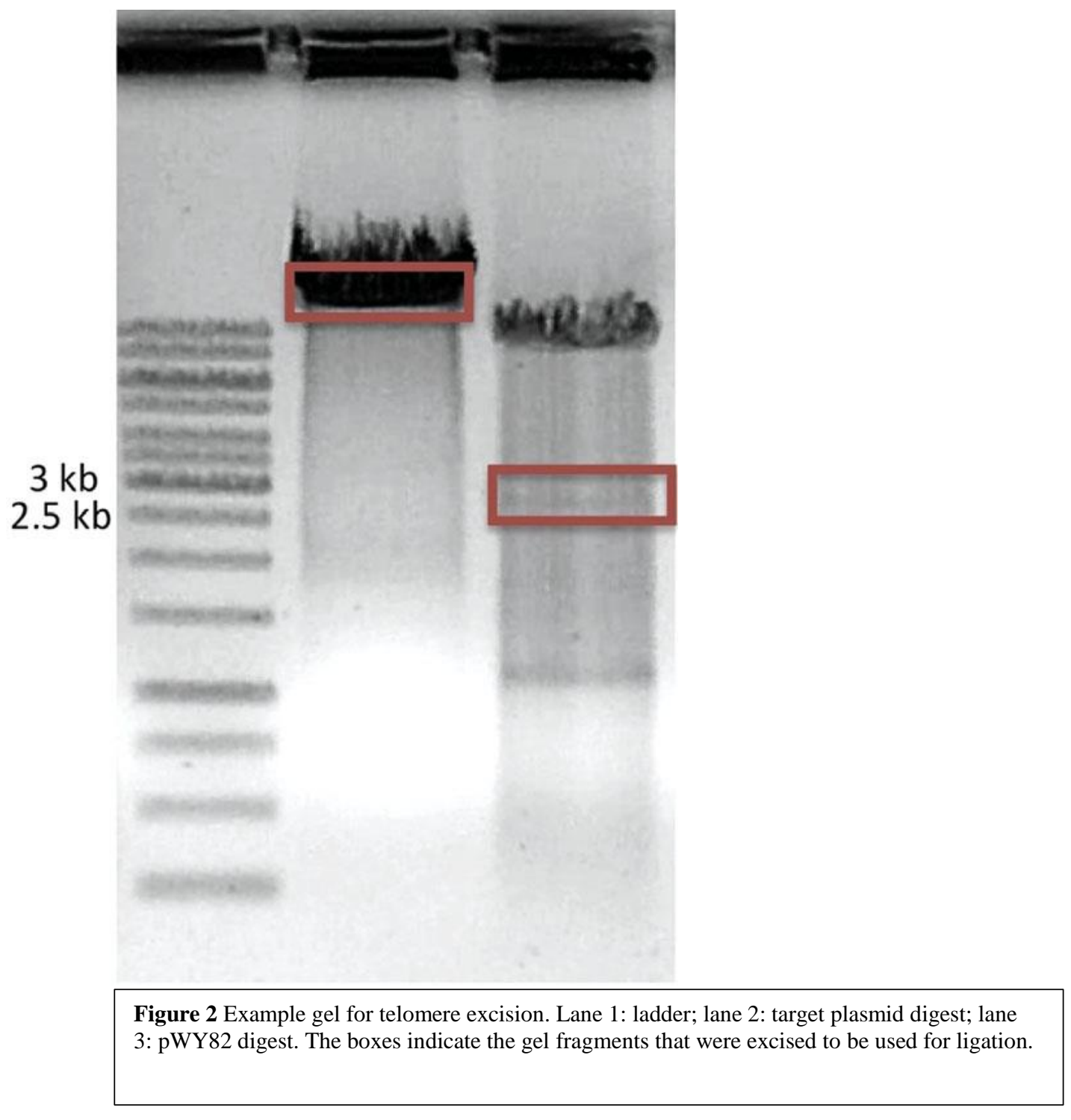


22. Load restriction digest completely into gel. Each digestion should be loaded into a separate well.

23. Load $6 \mu \mathrm{l} \mathrm{GeneRuler} 1 \mathrm{~kb}$ DNA Ladder into wells flanking restriction digest.

24. Run the gel in a $4^{\circ} \mathrm{C}$ cold room at $100 \mathrm{~V}$ until the lower band of loading dye is at the bottom of the gel. Caution should be taken whenmoving low-melting-point gels as they are extremely fragile.

25. Stain the gel in $0.5 \mu \mathrm{g} / \mathrm{ml}$ ethidium bromide for $30 \mathrm{~min}$. When shaking gel in ethidium bromide solution be sure to do so slowly to prevent the gel from breaking apart.

26. Visualize with UV light.

27. Estimate DNA concentration by comparing to ladder standard according to the manufacturer's instructions. Excise DNA and perform ligation.

28. Excise the uppermost telomere band and target plasmid backbone with a scalpel (Fig. 2), and place into a labeled 1.7-ml microcentrifuge tube. Plasmid and telomere bands should be placed in separate tubes. When excising bands be careful to remove only the portions containing DNA. Gel sections should be trimmed under UV light to ensure that only DNA-containing regions remain. 
29. Add $1 \mathrm{ml}$ nuclease-free water to the tubes, and place at $4^{\circ} \mathrm{C}$ overnight to remove excess salt.

30. Remove water and place in a $70^{\circ} \mathrm{C}$ water bath to melt.

31. Flick tube every minute until completely melted ( $\sim \mathrm{min})$. Tube is flicked to check visually if all of the solidified gel matrix has been melted.

32. Once agarose has completely melted, move to $37^{\circ} \mathrm{C}$ water bath.

33. Once the gel fragments have cooled to $37^{\circ} \mathrm{C}$ ( $\sim 5$ to $\left.10 \mathrm{~min}\right)$ prepare ligation mixture as follows:

$5 \mu \mathrm{l}$ ligase buffer

$100 \mathrm{ng}$ target plasmid gel fragment

$1 \mu \mathrm{l}(400 \mathrm{U})$ ligase

Telomere gel fragment to $50 \mu \mathrm{l}$

As the majority of the reaction contains agarose, the reaction will solidify at room temperature. Move quickly to prevent the mixture from prematurely solidifying. Gel fragments should be pipetted quickly so they are not allowed to cool. 
34. Mix by pipetting and incubate at room temperature overnight. When incubating at room temperature, the mixture should solidify. Transform ligation into Stbl4 cells

35. Add $1 \mathrm{ml}$ water to the tube and incubate at room temperature for $15 \mathrm{~min}$ to remove any excess salts. Flick the tube until the mixture floats freely in the water. Ensure gel fragments are completely suspended.

36. Thaw Stbl4 cells on ice. Telomere DNA is repetitive and tends to delete portions of the array, so Stbl4 cells are used to diminish this process.

37. Remove water from ligation reaction and add $50 \mu \mathrm{l}$ fresh nuclease-free water. Incubate at $70^{\circ} \mathrm{C}$ for $10 \mathrm{~min}$, flicking the tube every 2 min until completely melted.

38. To $40 \mu \mathrm{l}$ Stbl 4 cells, add $2 \mu$ ligation mixture and electroporate according to manufacturer's instructions.

39. Resuspend electroporated cells in SOC medium and shake in $15-\mathrm{ml}$ culture tubes at $30^{\circ} \mathrm{C}$ for $1.5 \mathrm{hr}$ at $250 \mathrm{rpm}$. SOC medium is super optimal broth with catabolic repression (Chan et al., 2013). 
40. Plate $100 \mu$ l transformation onto LB plates containing the appropriate antibiotics for transformation vector. It is beneficial to spread multiple plates to ensure there will be several colonies to screen in the following steps.

41. Incubate at $30^{\circ} \mathrm{C}$ until colonies appear. Due to the low temperature, colonies may not appear for $48 \mathrm{hr}$.

42. Screen colonies using colony hybridization or colony PCR. Colony hybridization (Sambrook et al., 1989) is the most efficient method for screening plates of colonies; we have found it to be the most sensitive. The oligonucleotide probe (TTTAGGG) 10 works extremely well and can be synthesized in either the 5' or 3' direction. If screening using colony PCR (Woodman, 2008), care must be taken when designing primers. PCR will often fail if attempting to amplify a product across the telomere insert, so it is not suggested to screen using primers that flank the entire insert. Instead, the forward primer should be designed 5' to the insertion point of the target plasmid and the reverse primer designed $5^{\prime}$ to the telomere repeats on the sequence unique to pWY82.

43. Confirm correct orientation of insert using standard sequencing. Standard Sanger sequencing will generally not be successful across the full length of the telomere insert and often will fail after only a few repeats. Detecting telomere insert size Once positive colonies have been detected, they need to be screened 
for insert size. While it is not known the exact size required for telomere truncation, it is suggested that the largest size possible be used during transformation. Size estimation is carried out using southern hybridization (Southern, 1975), with the donor plasmid pWY82 used as a positive control and the empty target plasmid as a negative control.

Prepare plasmid DNA

44. Inoculate $3 \mathrm{ml}$ of $2 \mathrm{X}$ YT medium containing appropriate antibiotics with pWY82, the original target plasmid, and positive colonies.

45. Shake at $250 \mathrm{rpm}$ for $8 \mathrm{hr}$ at $30^{\circ} \mathrm{C}$.

46. Prepare fresh $5 \mathrm{ml}$ cultures of $2 X \mathrm{YT}$ medium containing appropriate antibiotics with $100 \mu \mathrm{l}$ of the starter cultures.

47. Shake at $250 \mathrm{rpm}$ for $48 \mathrm{hr}$ at $30^{\circ} \mathrm{C}$.

48. Extract plasmid DNA using $4 \mathrm{ml}$ culture and the QIAprep Spin Miniprep Kit and elute using $40 \mu \mathrm{l}$ warm nuclease-free water.

49. Estimate the concentration of each extraction using a NanoDrop spectrophotometer. Digest DNA and perform electrophoresis 
50. Digest $1 \mu \mathrm{g}$ of each extraction with restriction endonucleases flanking the telomere insert as closely as possible in a $50 \mu$ reaction volume.

51. Pour a $1 \%(w / v)$ agarose gel in $1 X$ TAE using combs large enough to contain the entire restriction digest.

52. Upon completion of the digest, add $5 \mu \mathrm{l}$ of $6 \mathrm{X}$ loading dye to each reaction.

53. Load each digest into solidified gel, and run at $100 \mathrm{~V}$ in $1 \mathrm{X}$ TAE until lower marker band has reached the bottom of the gel.

54. Stain gel in $0.5 \mathrm{ug} / \mathrm{ml}$ ethidium bromide for $30 \mathrm{~min}$.

55. Visualize with UV light to confirm successful digest. Do not attempt to estimate telomere size at this point as it is unreliable. Transfer agarose gel and perform Southern hybridization

56. Transfer the digest to a nitrocellulose membrane by Southern transfer (Hoopes, 2012).

57. Hybridize with the radiolabeled oligonucleotide (TTTAGGG) ${ }^{10}$ and compare to the size standard to estimate insert size (Hoopes, 2012). 
58. Once the size of telomere inserts has been confirmed and if the vector is Agrobacterium-compatible, transform into Agrobacterium following the manufacturer's instructions.

\section{PRODUCTION OF TELOMERE FRAGMENTS VIA PCR}

Telomere fragments of various lengths can be produced by using polymerase chain reaction (PCR) with particular primers, which are listed below. The differing lengths of telomeres are created by annealing of primers to each other resulting in telomere repeats of various sizes. The resulting fragments of telomere can be visualized by gel electrophoresis, and particular sizes of telomere can be obtained by DNA gel extraction from an agarose gel. The obtained telomere DNA can then be used in particle bombardment with a construct of interest to create a minichromosome. This protocol has been adapted from a protocol that labels telomeres to make fluorescent probes (ljdo et al., 1991). The telomere fragments generated from this process include a wide range of sizes relative to the Basic Protocol. The Basic Protocol will result in a large amount of DNA of the specific size, while this protocol will give a larger range of sizes in much lower quantity.

\section{Materials}

Telomere DNA to be amplified Nuclease-free water 


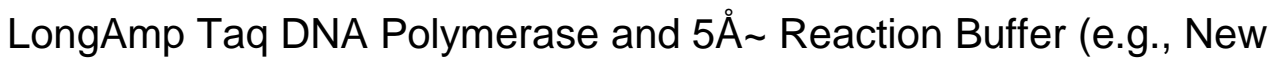

England

Biolabs, cat. no. M0323S)

Telomere primers $(1.25 \mu \mathrm{mol} / \mu \mathrm{l})$ :

Forward primer: 5_-(TTTAGGG)10-3_

Reverse primer: 5_-(CCCTAAA)10-3_

$10 \mathrm{mM}$ dNTP mix

Wizard SV Gel and PCR Clean-Up System (e.g., Promega, cat no. A9281)

Thermal cycler

Additional equipment and reagents for gel electrophoresis (Green and

Sambrook,2012)

1. Using a proofreading Taq polymerase, such as LongAmp Taq DNA Polymerase, assemble the PCR mix as follows:

$12 \mu \mathrm{l}$ nuclease-free water

$4 \mu \mathrm{l}$ of $5 \AA \AA$ LongAmp Taq buffer

$0.5 \mu \mathrm{l}$ forward telomere primer

$0.5 \mu \mathrm{l}$ reverse telomere primer

$2 \mu \mathrm{l}$ of $10 \mathrm{mM}$ dNTP mix

$1 \mu \mathrm{l}$ LongAmp Taq Polymerase

Volumes listed are per reaction and should be scaled up appropriately. It is recommended to make several reactions as DNA gel extraction will greatly reduce the amount of DNA obtained from each sample 
2. Carry out the PCR on a thermal cycler using the following amplification cycles:

5 cycles: $10 \sec 98^{\circ} \mathrm{C}$

$20 \sec 55^{\circ} \mathrm{C}$

$5 \sec 72^{\circ} \mathrm{C}$

5 cycles: $20 \sec 98^{\circ} \mathrm{C}$

$20 \sec 55^{\circ} \mathrm{C}$

$5 \sec 72^{\circ} \mathrm{C}$

5 cycles: $20 \sec 98^{\circ} \mathrm{C}$

$20 \sec 55^{\circ} \mathrm{C}$

$10 \sec 72^{\circ} \mathrm{C}$

5 cycles: $20 \sec 98^{\circ} \mathrm{C}$

$20 \sec 62^{\circ} \mathrm{C}$

$30 \sec 72^{\circ} \mathrm{C}$

Final step: $5 \min 72^{\circ} \mathrm{C}$

Hold: Indefinitely $4^{\circ} \mathrm{C}$ 


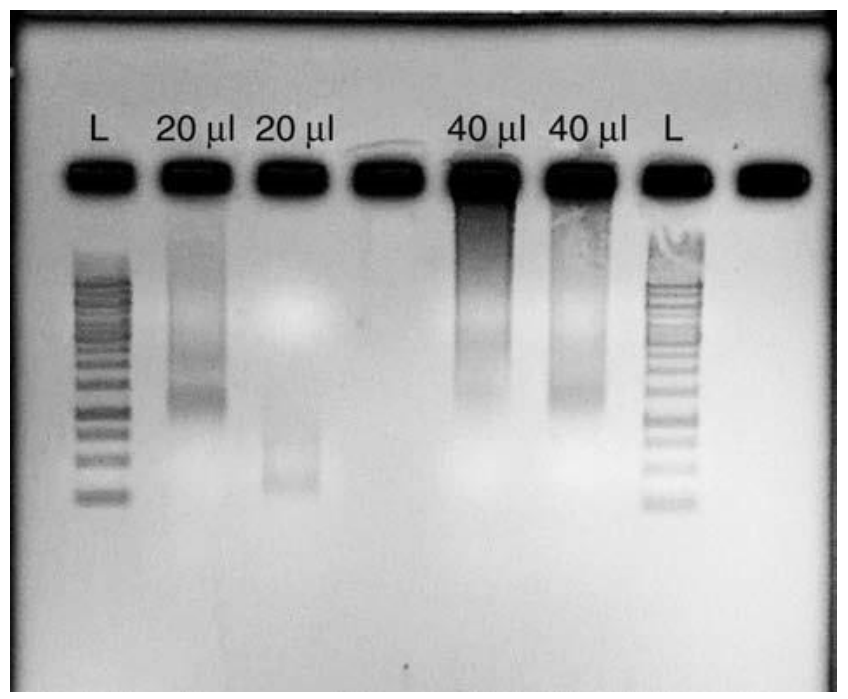

Figure 3 Telomere PCR example using $20 \mu$ l and $40 \mu$ reaction volumes. L represents the $1 \mathrm{~kb}$ ladder, and $20 \mu \mathrm{l}$ and $40 \mu \mathrm{l}$ each represent the reaction volume of each sample. 
The thermal cycler protocol will take $\sim 1 \mathrm{hr}$ to complete. Reactions can be stored at $4^{\circ} \mathrm{C}$ or $-20^{\circ} \mathrm{C}$ for later use.

3. Perform gel electrophoresis on reaction products to visualize telomere conglomerates. An example protocol has been described (Green and Sambrook, 2012). Telomere "smears" will be generated and will appear as in Figure 3.

4. Using a gel extraction kit such as Wizard SV Gel and PCR Clean-Up System, excise the gel piece corresponding to the desired size following the manufacturer's instructions. DNA extraction from a gel will dramatically reduce the amount of DNA present in the initial sample, which is why multiple reactions or large volume reactions are recommended. If one wishes to use the resulting telomere DNA for cloning, it should be eluted with nuclease-free water. Following the PCR and gel extraction, telomere DNA of a particular size is obtained. The DNA can be further processed, via blunt cutting restriction endonucleases, and cloned into a target vector. Alternatively, the telomere DNA can be used as free telomere for a cobombardment with the construct of interest. 
Telomere DNA can be stored at $-20^{\circ} \mathrm{C}$ for later use.

\section{DELIVERY OF TRANSGENE WITH TELOMERE ARRAYS INTO PLANTS}

After generating telomere arrays and either cloning them into a vector or not, these arrays can then be used for the generation of a minichromosome using telomere-mediated chromosomal truncation. There are two methods for accomplishing telomere-mediated chromosomal truncation: (1) particle bombardment utilizing gold particles and a gene gun and (2) Agrobacterium transformation. Agrobacterium transformation has been used for telomeremediated truncation by utilizing the natural ability of Agrobacterium to deliver DNA to the nucleus to integrate a telomere array-containing construct (Yu et al., 2006). Telomere-mediated truncation can be accomplished by particle bombardment either by simply delivering a transgene with attached telomere arrays or by cobombardment of thetransgene of interest with free telomere (Songstad et al., 1996; Frame et al., 2000; Gaeta et al., 2013). For cobombardment, a 1:1 molar ratio of construct of interest to telomere DNA has been used successfully for telomere-mediated chromosomal truncation (Gaeta et al., 2013). The protocols for both particle bombardment and Agrobacterium transformation are not changed by inclusion of telomere in the transformation. 


\section{REAGENTS AND SOLUTIONS}

Use deionized, distilled water in all recipes and protocol steps.

EDTA (pH 8.0), $0.5 \mathrm{M}$ For 1 liter of stock solution, add $186.1 \mathrm{~g}$ EDTA to $800 \mathrm{ml}$ ddH2O. Adjust the $\mathrm{pH}$ to 8 with $\mathrm{NaOH}$. Bring final volume to 1 liter with ddH2O, and sterilize by autoclaving. Store at room temperature for several years. EDTA will not begin to dissolve until the $\mathrm{pH}$ approaches 8 .

LB plates, For $500 \mathrm{ml}$ dissolve $12.5 \mathrm{~g}$ Luria broth (e.g., Fisher Scientific, cat. no. BP9723-2) and $6 \mathrm{~g}$ agar in $400 \mathrm{ml} \mathrm{ddH} 2 \mathrm{O}$. Bring to $500 \mathrm{ml}$ with ddH2O and autoclave for $20 \mathrm{~min}$. Place in $50^{\circ} \mathrm{C}$ water bath until completely cooled, and then add appropriate antibiotics. Gently mix and pour thin layer into petri dishes. Store at $4^{\circ} \mathrm{C}$ for up to 1 month.

Yeast extract tryptone (YT) medium, 2X, For $500 \mathrm{ml}$ of culture dissolve $15.5 \mathrm{~g}$ of 2X YT (e.g., Sigma-Aldrich, cat. no. Y2377) in $400 \mathrm{ml} \mathrm{ddH2O.} \mathrm{Bring} \mathrm{final} \mathrm{volume}$ to $500 \mathrm{ml}$ and autoclave for $20 \mathrm{~min}$. Store at room temperature for several years.

TAE, 50X, For a 1 liter stock solution add $242 \mathrm{~g}$ tris base, $57.1 \mathrm{ml}$ acetic acid, and $100 \mathrm{ml}$ 0.5M EDTA (see recipe) to $500 \mathrm{ml} \mathrm{ddH2O}$. Dissolve tris base and bring to 1 liter with ddH2O. Store at room temperature for several years. 
To prepare 1 liter of $1 \mathrm{X}$ TAE, dilute $20 \mathrm{ml}$ of $50 \AA \AA \sim \mathrm{TAE}$ in $980 \mathrm{ml} \mathrm{ddH} 20$

\section{COMMENTARY}

\section{Background Information}

Telomere truncation has been successfully used to create engineered minichromosomes in maize (Yu et al., 2007; Gaeta et al., 2011, 2013). Regenerated transformant plants may possess a minichromosome, or they may possess an insertion without truncation or a translocation (Yu et al., 2006). Fluorescence in-situ hybridization (FISH) is useful for identifying minichromosomes and can detect non-truncating transgene insertions and translocations as well. A protocol for chromosome preparation has been described (see McCaw et al., 2016a) and for FISH (see McCaw et al., 2016b). A detailed video is also available at https://www.youtube.com/watch? v=aOX0DrcFVE. A B-specific repeat probe in green will hybridize strongly to the centromere of a B chromosome, and a small-target red probe, because it is the most sensitive, made from the transgene plasmid should be visible at the end of the chromosome where truncation has occurred. The signal may appear to be slightly proximal of the end of the chromosome in some images because of the coiling of chromatin during condensation (Yu et al., 2006). If no B-derived minichromosome is present, a simpler all green karyotyping cocktail (see McCaw et al., 2016b) can be used with a single gene probe prepared from the transgene in red. This probe combination can identify most $A$ chromosomes, and transgene 
signals should be visible as two red dots, one on each sister chromatid.

Nontruncating A chromosome insertions, translocations (Gaeta et al., 2011), and minichromosomes derived from A chromosomes (Yu et al., 2007; Gaeta et al., 2013) can all be identified by these probes.

Not all maize lines are equal in terms of transformability. Two types of embryogenic calli can be induced from immature maize embryos, known as Type-I and Type-II. Type- I is slow growing, dense, and generally not preferable for transformation. Type-II is faster growing and most importantly is friable: small bits of callus can easily be teased apart from each other and regenerated quickly into more calli derived from the small clump. Because of this, a transformed callus can be more easily separated from an untransformed callus and proliferated into a more homogeneous culture than with a Type-I callus. From the resultant uniform cultures, whole transgenic plants can be regenerated with little chance of untransformed sectors. Before Hi-II was developed, the Type-II callus was generally derived from A188, B73, or an F1 of the two lines. These crosses had a low rate of Type-II callus initiation and/or were difficult to maintain friable callus. Hi-II is a hybrid selected to have a high ( 100\%) rate of Type-II embryogenic callus formation (Armstrong et al., 1991). "Hi-II A" and "Hi-II B" were selected from separate A188 X B73 F2 embryos to have a high rate of Type-II callusormation. The "A" and "B" parents can be crossed to yield more vigorous F1 hybrid embryos for transformation.

\section{Critical Parameters}




\section{Telomere ligation}

Telomere-containing plasmids should be maintained in Stbl4 cells, which must be grown at $30^{\circ} \mathrm{C}$. Due to a lower temperature, these cells often take $48 \mathrm{hr}$ to grow. Basic Protocol step 11: Choice of restriction endonucleases is imperative to successful cloning reactions. If possible, choose endonucleases that will not result in blunt ends.

Basic Protocol step 15: pWY82 does not behave as expected in an agarose gel. Telomere repeats will form secondary structures, which run lower than expected and often form more bands than would be expected if fully extracted from the backbone.

Basic Protocol step 42: Colony hybridization (Sambrook et al., 1989) is the most efficient method for screening plates of colonies, and we have found it to be the most sensitive. The oligonucleotide probe (TTTAGGG) ${ }^{10}$ works extremely well and can be synthesized in either the 5' or 3' direction. If screening using colony PCR, care must be taken when designing primers. PCR will often fail if attempting to amplify a product across the telomere insert, so it is not suggested to screen using primers that flank the entire insert. Instead, the forward primer should be designed $5^{\prime}$ to the insertion point of the target plasmid, and the reverse primer should be designed 5 ' to the telomere repeats on the sequence unique to pWY82.

Basic Protocol step 43: Generally, sequencing reactions will fail after 10 to 15 telomere repeats. 
Basic Protocol step 46: Depending on how many colonies there are to screen, it may be useful to prepare 10 - or $15-\mathrm{ml}$ cultures. To screen for telomere size, $4 \mathrm{ml}$ of each is used and the remainder pelleted and saved for future large-scale preps.

Basic Protocol step 57: Multiple bands will be apparent on the Southern hybridization exposure due to the telomere secondary structure. In addition, a band will generally be visible due to hybridization to the uncut plasmid DNA background.

\section{Production of telomere fragments by PCR}

We have observed that the concentration of the primers can affect the size of the telomere smears produced. A higher concentration results in smaller fragments, and a lower concentration (the one recommended in this unit) gives larger fragments. Several PCRs should be prepared because extracting DNA from an agarose gel will result in a much lower amount than input. Larger volume reactions may also be used to mitigate the effects of DNA gel extraction.

\section{Troubleshooting}

\section{In-gel ligation}

As the telomere repeats are generally unstable, the efficiency of the cloning procedure is generally quite low. Similarly, the presence of telomeres will often 
reduce the yield of DNA extractions once the procedure is successful. If no successful insertions are found, ensure that the DNA is fully intact prior to cloning. It is helpful to run an uncut plasmid control during steps 11 through 15 of the Basic Protocol to ensure that the restriction endonucleases are functional and the DNA is not degraded. Varying the amount of telomere DNA that is added to the reaction can greatly alter the success of the cloning reaction.We have found that reducing or increasing the amount of input telomere DNA by even $5 \mu \mathrm{l}$ can greatly improve the chance of success. When screening for positive colonies, care should be taken during primer design. Due to the complexity of the telomere repeats most polymerases will fail when attempting to perform PCR across them. As a result, care should be taken to avoid using primers that would rely on a product containing telomere repeats. In our laboratory, we generally will use a primer in the target plasmid and one just 5' of the telomere repeats in the sequence that was unique to pWY82.

\section{Anticipated Results}

Successful completion of the in-gel ligation protocol (Basic Protocol) should result in a large amount of telomere repeat inserted into the target vector. Completion of the telomere PCR protocol (Alternate Protocol) should result in enough telomere DNA of the desired size to use for cobombardment into plants or for blunt cloning into the desired vector.

\section{Time Considerations}




\section{Basic Protocol: In-gel ligation}

DNA preparation (steps 1 through 9 ) can generally be performed in 6 to 7 days. Plates can be stored at $4^{\circ} \mathrm{C}$ for weeks after colonies are observed. Pelleted DNA in step 8 can be stored at $4^{\circ} \mathrm{C}$ up to 3 days before processing through columns. Steps 10 through 15 generally take 2 to $3 \mathrm{hr}$, and steps 16 through 27 take 3 to 4 hr. Two overnight incubations are performed in steps 28 through 34 . Steps 35 through 43 take $2.5 \mathrm{hr}$ to prepare cells and 2 days to grow on plates. Detecting telomeres can be performed in 2 to 3 days by colony hybridization. Preparation of DNA and electrophoresis (steps 44 through 57 ) can generally be performed in 3 days. Southern hybridization can be performed and analyzed in 2 days.

\section{Alternate Protocol: Production of telomere fragments via PCR}

PCR assembly and performing the thermal cycler protocol (steps 1 and 2) will take about $2 \mathrm{hr}$. Gel electrophoresis of the completed PCR (step 3) takes about $1 \mathrm{hr}$ to complete. Extraction of DNA from a gel (step 4) takes about $1 \mathrm{hr}$. The total time for the Alternate Protocol is $4 \mathrm{hr}$.

\section{Acknowledgements}

Work on this topic is supported by National Science Foundation grant IOS1339198. 


\section{Literature Cited}

Ananiev, E.V, Wu, C., Chamberlin, M., Svitashev, S., Schwartz, C., GordonKamm, W., and Tingey, S. 2009. Artificial chromosome formation in maize (Zea mays L.). Chromosoma118:157-177. doi: 10.1007/s00412-008-0191-3.

Armstrong, C.L., Green, C.E., and Phillips, R.L. 1991. Development and availability of germplasm with High Type-Il culture formation response. Maize Genet. Coop. Newsl. 65: 92-93.

Birchler, J.A. and Han, F. 2009. Maize centromeres: Structure, function, epigenetics. Annu. Rev. Genet. 43: 287-303.

Carlson, S.R., Rudgers, G.W., Zieler, H., Mach, J.M., Luo, S., Grunden, E., Krol, C., Copenhaver, G.P., and Preuss, D. 2007. Meiotic transmission of an in vitroassembled autonomous maize minichromosome. PLoS Genet. 3:e179. doi: 10.1371/journal.pgen.0030179.

Chan, W., Verma, C.S., David, P., and Gan, S.K. and factors affecting the transformation of Escherichia coli. Biosci. Rep. 33:e00086. doi: 10.1042/BSR20130098.

Farr, C., Fantes, J., Goodfellow, P., and Cooke, H. 1991. Functional reintroduction of human telomeres into mammalian cells. Proc. Natl. Acad. Sci. U.S.A. 88:70067010. doi: $10.1073 /$ pnas.88.16.7006.

Frame, B.R., Zhang, H., Cocciolone, S.M., Sidorenko, L.V, Dietrich, C.R., Pegg, S.E., Zhen, S., Schnable, P.S., and Wang, K. 2000. Production of transgenic maize from bombarded type II callus: Effect of gold particle size and callus morphology on transformation efficiency. In Vitro Cell. Dev. PI. 36:21-29. doi: 10.1007/s11627000-0007-5.

Gaeta, R., Masonbrink, R., Krishnaswamy, L., Zhao, C., and Birchler, J. 2012. Synthetic chromosome platforms in plants. Annu. Rev. Plant Biol. 63:307-330. doi: 10.1146/ annurev-arplant-042110-103924.

Gaeta, R.T., Danilova, T.V, Zhao, C., Masonbrink, R.E.,McCaw,M.E., andBirchler, J.A. 2011. Recovery of a telomere-truncated chromosome via a compensating translocation in maize. Genome 54:184-195. doi: 10.1139/G10-108.

Gaeta, R., Masonbrink, R., Zhao, C., Sanyal, A., Krishnaswamy, L., and Birchler, J. 2013. In vivo modification of a maize engineered minichromosome. Chromosoma 122:221-232. doi: 10.1007/s00412-013-0403-3.

Gibson, D.G., Young, L., Chuang, R.-Y., Venter, J.C., Hutchison, C.A, and Smith, H.O. 2009. Enzymatic assembly of DNA molecules up to several hundred kilobases. Nat. Methods 6:343-345. doi: 10.1038/nmeth.1318. 
Green,M.R. and Sambrook, J. (eds.) 2012. Molecular Cloning: A Laboratory Manual, 4th ed. Cold Spring Harbor Laboratory Press, Cold Spring Harbor, N.Y. Harrington, J.J., Van Bokkelen, G., Mays, R.W., Gustashaw, K., and Willard, H.F. 1997. Formation of de novo centromeres and construction of first-generation human artificial microchromosomes. Nat. Genet. 15:345-355. doi: 10.1038/ng0497-345.

Heller, R., Brown, K.E., Burgtorf, C., and Brown, W.R. 1996. Mini-chromosomes derived from the human $\mathrm{Y}$ chromosome by telomere directed chromosome breakage. Proc. Natl. Acad. Sci. U.S.A. 93:7125-7130. doi: 10.1073/pnas.93.14.7125.

Hoopes, L.L.M. 2012. Nucleic acid blotting: Southern and northern. Curr. Protoc. Essen. Lab. Techn. 6:8.2.1-8.2.26. doi: 10.1002/9780470089941.et0802s6. ljdo, J.W., Wells, R., Baldini, A., and Reeders, S.T. 1991. Improved telomere detection using a telomere repeat probe (TTAGGG)n generated by PCR. Nucl. Acids Res. 19:4780. doi: 10.1093/ nar/19.17.4780.

Kapusi, E.,Ma, L., Teo, C.H., Hensel, G., Himmelbach, A., Schubert, I., Mette, M.F., Kumlehn, J., and Houben, A. 2012. Telomere-mediated truncation of barley chromosomes. Chromosoma 121:181-190. doi: 10.1007/s00412-011-0351-8.

McCaw, M., Swyers, N., Graham, N., Cody, J., Zhao, C., and Birchler, J., 2016a. Preparation of chromosomes from Zea mays. Curr Protoc. Plant Biol. 1:501-508. doi: 10.1002/cppb. 20032.

McCaw, M., Graham, N., Cody, J., Swyers, N., Zhao, C., and Birchler, J. $2016 \mathrm{~b}$. Fluorescence in situ hybridization to maize (Zea mays) chromosomes. Curr. Protoc. Plant Biol. 1:529-544. doi: 10.1002/cppb.20033.

Murray, A.W. and Szostak, J.W. 1983. Construction of artificial chromosomes in yeast. Nature 305:189-193. doi: 10.1038/305189a0.

Nelson, A.D., Lamb, J.C., Kobrossly, P.S., and Shippen, D.E. 2011. Parameters affecting telomere-mediated chromosomal truncation in Arabidopsis. Plant Cell 23:2263-2272. doi: 10.1105/tpc.111.086017.

Potter, H. and Heller, R. 2010. Transfection by electroporation. Curr. Protoc. Mol. Biol. 92:9.3.1-9.3.10. doi: 10.1002/0471142727. mb0903s9.

Sambrook, J., Fritsch, E. F., and Maniatis, T. (eds.) 1989. Molecular Cloning: A Laboratory Manual, 2nd ed. Cold Spring Harbor Laboratory Press, Cold Spring Harbor, N.Y. 
Shendure, J. A., Porreca, G. J., Church, G. M., Gardner, A. F., Hendrickson, C. L., Kieleczawa, J., and Slatko, B. E. 2011. Overview of DNA sequencing strategies. Curr. Protoc. Mol. Biol. 96:7.1.1-7.1.23. doi: 10.1002/0471142727.mb0701s96.

Shizuya, H., Birren, B., Kim, U.J., Mancino, V., Slepak, T., Tachiiri, Y., and Simon, M. 1992. Cloning and stable maintenance of 300- kilobase-pair fragments of human DNA in Escherichia coli using an F-factor-based vector. Proc. Natl. Acad. Sci. U.S.A. 89:8794-8797. doi: 10.1073/pnas.89.18.8794.

Songstad, D.D., Armstrong, C.L., Petersen, W.L., Hairston, B., and Hinchee, M.A.W. 1996. Production of transgenic maize plants and progeny by bombardment of Hi-Il immature embryos. In Vitro Cell. Dev. Pl. 32:179-183. doi: 10.1007/BF02822763.

Southern, E.M. 1975. Detection of specific sequences among DNA fragments separated by gel electrophoresis. J. Mol. Biol. 98:503-517.

Teo, C.H., Ma, L., Kapusi, E., Hensel, G., Kumlehn, J., Schubert, I., Houben, A., and Mette, M.F. 2011. Induction of telomeremediated chromosomal truncation and stability of truncated chromosomes in Arabidopsis thaliana. Plant J. 68:28-39. doi: 10.1111/ j.1365-313X.2011.04662.x.

Woodman, M.E. 2008. Direct PCR of intact bacteria (colony PCR). Curr. Protoc. Microbiol. 9:A.3D.1-A.3D.6. doi: 10.1002/9780471729259.mca03ds9.

$\mathrm{Xu}, \mathrm{C} .$, Cheng, Z., and Yu,W. 2012. Construction of rice mini-chromosomes by telomere-mediated chromosomal truncation. Plant J. 70:1070-1079. doi: 10.1111/j.1365-313X.2012.04916.x.

Yu,W., Lamb, J.C., Han, F., and Birchler, J.A. 2006. Telomere-mediated chromosomal truncation in maize. Proc. Natl. Acad. Sci. U.S.A. 103:17331-17336. doi: 10.1073/pnas.0605750103.

Yu, W., Han, F., Gao, Z., Vega, J.M., and Birchler, J.A. 2007. Construction and behavior of engineered minichromosomes in maize. Proc. Natl. Acad. Sci. U.S.A. 104:8924-8929. doi: 10.1073/ pnas.0700932104. 


\section{Appendix 3: Preparation of Chromosomes from Zea}

\section{mays}

Note: The information in this chapter was published under the title:

McCaw M.E., Swyers N.C., Graham N.D., Cody J.P., Zhao C., Birchler J.A. (2016) preparation of chromosomes from Zea mays. Curr. Protoc. Plant Biol. 1:501-509. doi: 10.1002/cppb.20032 


\section{Summary}

High-quality preparations of chromosomes are useful for many purposes.

To prepare metaphase chromosome spreads in maize, root tips are harvested and treated with nitrous oxide to stop cell division at metaphase before being fixed in acetic acid. This process allows a high number of condensed chromosome spreads to be obtained at the end of the procedure. To prepare chromosome spreads from various stages of meiosis, anthers are first fixed before being examined for developmental stage. Cells are digested with a mixture of enzymes before the chromosomes are dropped onto glass sides and fixed under UV light.

\section{Introduction}

These protocols describe how mitotic metaphase and meiotic nuclei can be maximized for visualization. The resulting samples are ideal for labeling with fluorescence in situ hybridization or can be viewed with a compound microscope. These protocols describe the collection and preparation of maize tissue samples as well as glass slide preparation of those tissues. The metaphase protocol (see Basic Protocol 1) describes how maize root tips are harvested and prepared so that a large number of metaphase nuclei are present for chromosome visualization. The meiosis protocol (see Basic Protocol 2) describes how maize tassels are harvested and anthers prepared on slides for visualization. 


\section{Basic Protocol 1: Preparation of Metaphase Chromosomes}

Distinguishing individual maize chromosomes and specific regions on

those chromosomes can be difficult unless nuclei are visualized at specific times during mitosis. Capturing nuclei in metaphase facilitates chromosome identification. Preparing slides that have the maximum number of nuclei in metaphase also allows for further analyses using methods such as fluorescence in situ hybridization (FISH) easier. This protocol will describe how to prepare root tips of maize plants for visualization of metaphase chromosomes.

\section{Materials:}

- Maize seeds

- Nitrous oxide gas (local medical gas supply company)

- $90 \%$ glacial acetic acid, store at $4^{\circ} \mathrm{C}$

- $70 \%$ ethanol $1 \times$ citric buffer (see recipe)

- Digestive enzyme solution (see recipe)

- Methanol Ice bucket containing ice

- Paper towels or Kimwipes

- $30^{\circ} \mathrm{C}$ incubator

- Razor blade or scalpel, tweezers, blunt-ended stainless steel dissecting probe 0.6 - or $1.5-\mathrm{ml}$ tubes

- Pressure chamber for nitrous oxide treatment (custom manufactured; https://birchler.biology.missouri.edu/wp-content/uploads/2015/07/Maize- 
Karyotyping-and-FISH-Manual_2015.pdf, page 54) Filter paper (Whatman no. 1 or thicker)

- $37^{\circ} \mathrm{C}$ water or dry bath Humid chamber Glass microscope slides $(1 \times 3-$ in. $)$ Compound microcope UV cross-linker (120 to $125 \mathrm{~mJ} / \mathrm{cm} 2$ )

\section{Prepare root tips:}

1. To acquire root tips for slide preparation, germinate maize seeds on wet paper towels or Kimwipes and then take the roots. Roots can also be harvested directly from young plants or even adult plants before flowering. Primary roots should be preferentially harvested as they have an abundance of mitotic cells, though roots which appear similar to primary roots from older plants will also work. If germinating seedlings on paper towels, incubate seedlings for 2 to 3 days at $30^{\circ} \mathrm{C}$ before harvesting.

2. Cut $\sim 1.5 \mathrm{~cm}$ off of the tip of the root and place into a $0.6-$ or $1.5-\mathrm{ml}$ tube. Pierce top of tube with dissecting probe to allow gas to enter. Lightly mist the inside of the tube with water to keep the root tip moist. Be careful to not saturate the roots in water, as this may prevent the roots from being exposed to the gas.

3. Treat tubes containing root tips with $\mathrm{N} 2 \mathrm{O}$ in a chamber for 1 to $3 \mathrm{hr}$ (Kato, 1999).

The longer the root tip is exposed to $\mathrm{N} 2 \mathrm{O}$, the more compact the chromosomes are in the nuclei. 
Kato (1999) compared nitrous oxide treatment to other methods of arresting metaphase. Nitrous oxide is the most effective method, but other treatments can be used.

4. After $\mathrm{N} 2 \mathrm{O}$ treatment, immediately remove tubes and place on ice, then treat root tips with $90 \%$ acetic acid for $10 \mathrm{~min}$.

This step achieves fixation of the root tips.

\section{Wash root tips:}

5. Remove acetic acid solution and wash root tips with $70 \%$ ethanol and then place root tips into new tubes containing fresh $70 \%$ ethanol.

Root tips can be stored for months to 1 year or more in $70 \%$ ethanol at $-20^{\circ} \mathrm{C}$

6. Remove ethanol and replace with $1 \times$ citric buffer, then allow roots to soak $\sim 15 \min$.

Distilled water can be used in place of $1 \times$ citric buffer.

7. Lay root tips onto filter paper and gently wipe in the direction of the root cap to remove the substance on the cap. Wiping away this substance allows for a better digestion of the root tip.

\section{Digest root tips:}


8. Cut off 0.7 to $1.0 \mathrm{~mm}$ of the distal tip of the root tip, where the actively dividing meristem is located, and transfer to a $0.6-\mathrm{ml}$ tube containing $20 \mu \mathrm{l}$ of enzyme solution on ice.

Attempt to cut root tips the same size so as to keep digestion time for each root tip consistent.

The meristem has an opaque, whitish appearance at the tip of the root.

9. Incubate tubes 45 to 60 min in a $37^{\circ} \mathrm{C}$ water bath to allow for digestion.

Digestion time varies depending on size of root tip, age of enzyme, as well as other variables. Average digestion time is $\sim 50 \mathrm{~min}$.

10. Remove tubes from incubation and place on ice.

11. Rinse tubes two times with $70 \%$ ethanol, taking care not to destroy or throw away digested root tip. Carefully dripping the ethanol along the inside of the tube at an angle helps prevent destruction of the digested root tip. If the root tip is destroyed, it is possible to centrifuge the tube of digested material and recover enough for a slide. Removing the ethanol after washes can be achieved by careful pouring or by careful pipetting, being sure to not destroy the fragile root tip.

12. Add $30 \mu$ of $90 \%$ acetic acid $10 \%$ methanol mixture, or $100 \%$ acetic acid, to the tubes replacing the ethanol.

\section{Break up root tip and place onto slides:}


13. Use a blunt-ended dissecting probe to break up the root tip by smashing the root tip against side of tube. Wipe probe clean between samples to prevent contamination. Stir or tap tube to resuspend cells. Keep cells on ice.

14. Prepare a humid chamber and place labeled slides into the chamber. Humid chamber can be a simple cardboard box layered with wet paper towels. Place slides on a dry surface elevated above the wet paper towels. Square wooden dowels work well for this purpose. Cover box with damp Kimwipe to create a humid environment for the cell suspension to spread.

15. On each slide, drop 5.5 to $9.0 \mu$ of the cell suspensions.

The drop of cell suspension should spread in an even circle from where it was dropped. If the drop does not spread, then most likely it is not humid enough. Breathe gently on the drop to help it spread. Not removing enough of the $70 \%$ ethanol can also cause spreading problems. A dirty slide usually causes erratic spreading but could also potentially prevent spreading.

16. After the slides have dried, view slides under a compound microscope to determine their quality based on the nature of the chromosome spreads. Sufficient separation of chromosomes is desired, as well as a lack of cytoplasm around the chromosome spreads. This makes for a clearer hybridization with fluorescent probes.

17. Once slides have been checked for chromosome spreads, cross-link them using a UV cross-linker at optimum setting for a total energy of 120 to 125 $\mathrm{mJ} / \mathrm{cm} 2$. 
The prepared slides can now be labeled with fluorescent probes if desired, stored at $4^{\circ} \mathrm{C}$, or stored for several months at $-20^{\circ} \mathrm{C}$.

\section{Basic Protocol 2: Preparation of Meiotic Chromosomes}

Maize chromosomes may be visualized throughout meiosis. For a complete description of the stages of meiosis with pictures, refer to Neuffer et al. (1997). Microspores are obtained from the anthers of developing tassels. To determine whether a tassel is at the desired level of maturity, florets can be obtained and preliminarily staged before removing and fixing a whole tassel as per the Support Protocol. The same method can also be used to obtain florets for chromosome preparation if a subsequent self-cross or male out-crossing of the plant is desired. The most mature anthers are found $\sim 1 / 3$ of the way down from the tip of the main spike of the tassel. Anthers proximal to this point will be more mature than those distal. The tassel branches mature later than the main spike and follow this same general pattern of development. This is also the pattern observed in the timing of pollen shedding.

\section{Materials:}

- Plant with developing tassel

- 3:1 fixative: 3 parts $95 \%$ ethanol/1 part 100\% acetic acid

- $70 \%$ ethanol 
- Acetocarmine stain (see recipe)

- Alcohol lamp

- Deionized water or $1 \times$ citric buffer (see recipe)

- Digestive enzyme solution (see recipe)

- $100 \%$ acetic acid or $90 \%$ acetic acid

- $\quad 50$-ml tubes

- Scalpel and forceps

- Plastic petri dishes

- Glass slides and $22 \times 22-\mathrm{mm}$ glass coverslips

- Iron dissecting probe

- Compound light microscope

- 2-ml tubes

- $\quad 37^{\circ} \mathrm{C}$ water bath

- $\quad$ Lint-free tissues

- Blunt-end, stainless-steel dissecting probe

- Humid chamber (plastic chamber with wet paper towels on the bottom and sides with square wooden dowels)

- UV cross-linker (total energy of 120 to $125 \mathrm{~mJ} / \mathrm{cm} 2$ )

\section{Fix tassel:}


1. Remove developing tassel from plant and place into an empty $50-\mathrm{ml}$ tube, fill the remaining volume of the tube with 3:1 fixative, seal tube, and fix $24 \mathrm{hr}$ at $4^{\circ} \mathrm{C}$.

2. Decant fixative and replace with $70 \%$ ethanol; keep on ice for 15 min. Decant ethanol and replace with fresh $70 \%$ ethanol.

The tassel may now be stored up to 1 year or more at $-20^{\circ} \mathrm{C}$.

\section{Stage anthers:}

3. Remove tassel or tassel branch and place in a petri dish. Fill dish with $70 \%$ ethanol.

4. Remove a floret from the tassel using forceps being careful not to crush the anthers inside.

It is helpful to mark the position from which the anther is harvested as others around it will be at a similar developmental stage and this can help infer relative development of anthers proximal or distal to the most mature point mentioned above.

5. Dissect floret in the lid of a plastic petri dish partially filled with $70 \%$ ethanol; be careful not to cut into anthers. There should be two sets of three anthers. All three anthers in each set will be at the same developmental stage and the longer group will be more developed than the smaller group. Separate the two groups then remove one anther from a group of three and place it onto a glass slide and add a drop of acetocarmine. 
6. In the drop of acetocarmine, cut the anther widthwise with a sharp scalpel. Use the side of an iron dissecting probe to roll from the tip of each half of the anther towards the cut end. Remove the husk of the anther with forceps.

The contents of the anther should extrude out of the cut end like toothpaste out of a tube.

7. Apply a glass coverslip to the drop of acetocarmine and anther contents, then heat the slide over an ethanol lamp but not to the boiling point of the acetocarmine.

8. Use a compound light microscope to determine the stage of meiosis of the anther by comparing it to the diagrams in Neuffer et al. (1997).

9. Label a $2-\mathrm{ml}$ tube with the identified stage of meiosis and fill with $70 \%$ ethanol. Use this tube to store the two remaining anthers that match the identified one.

These anthers can be stored for 1 year or more at $-20^{\circ} \mathrm{C}$.

\section{Digest anthers:}

10. Soak anthers in $1.5 \mathrm{ml}$ deionized water or $1 \times$ citric buffer for $10 \mathrm{~min}$. Residual ethanol can inhibit the enzyme in the next step; therefore, washing a second time with deionized water is recommended.

11. Separate anthers from each other and any somatic floret tissue on a plastic petri dish lid in deionized water or $1 \times$ citric buffer, or without water or buffer if performed quickly enough that the anthers do not begin to dry out. 
12. Place each individual anther into a tube containing $20 \mu$ enzyme solution and keep on ice.

Ensure that the anther is at the bottom of the enzyme solution to ensure digestion from all sides.

13. Incubate tubes 30 to $60 \mathrm{~min}$ in a $37^{\circ} \mathrm{C}$ water bath.

The digestion time depends on a number of factors including genotype, age, manufacturer of enzyme, and stage of meiosis. A digestion time of 50 min is a starting point, but the digestion time may need to be adjusted if microspores are over or under digested. Under digested microspores will have a cell membrane surrounding them.

14. Wash digested anther two times with $70 \%$ ethanol. The digested anther is fragile. Run the ethanol down the side of the tube to avoid breaking the anther apart and losing the microspores. If the anther is disrupted, centrifuging the ethanol-filled tube in a microcentrifuge will help recover the microspores.

The ethanol can be carefully poured out without losing the digested anther. If the anther is stuck to the bottom of the tube, pouring is relatively easy. If the anther is moving freely in the tube, attempt to remove most of the ethanol, then twist the tube as it is gently tilted to pour. This will eventually cause the surface of the ethanol to pin the anther against the side of the tube and it should stick and allow the ethanol to be rotated away from it and poured off. 
Digested anthers can be stored in the second $70 \%$ ethanol wash overnight at $-20^{\circ} \mathrm{C}$.

\section{Prepare slides:}

15. Pour off the $70 \%$ ethanol and set the tube upside down on a lint-free tissue to remove as much ethanol as possible. Tap the tube gently onto the lint-free tissue to remove residual ethanol.

16. Add $30 \mu \mathrm{l}$ of $100 \%$ acetic acid or $90 \%$ acetic acid $/ 10 \%$ methanol to the digested anther and crush the anther against the side of the tube with a blunt-end, stainless-steel dissecting probe with a rolling motion. Break apart the digested anther as well as possible.

Wipe probe thoroughly with a lint-free tissue between samples to avoid contamination.

17. Flick the tube to further separate and suspend cells, then drop $8 \mu \mathrm{l}$ of the suspended cell solution onto a glass slide in a humid chamber and allow it to dry.

The drop of cell suspension should spread in an even circle from where it was dropped. If the drop does not spread, then the chamber is not humid enough and breathing gently on the drop will help it spread. Not removing enough of the $70 \%$ ethanol can also cause problems for spreading. A dirty slide usually causes erratic spreading but could also potentially prevent spreading. 
18. Check slides with a light microscope to ensure proper digestion and correct meiotic staging.

Depending on the stage of meiosis, there are different criteria for proper digestion. In every stage before telophase I, the entire cell wall of the pollen grains should be digested and very little to no cytoplasm should be visible. The internal cell walls formed during telophase I and II cannot be digested before the rest of the cell becomes over-digested. In these cases, a good digestion will be one in which the nucleus or chromosomes are relatively free of cytoplasm, yet still paired with their sister cells across a barrier of cytoplasm and cell wall.

19. Irradiate slides in a UV cross-linker at Optimal Crosslink setting.

Slides can now be viewed for the respective purpose including probed fluorescently as per McCaw et all., (2016)

\section{Support Protocol 2: Determining When to Harvest a Tassel}

It is generally preferable to determine the stages of meiosis present in a tassel before harvesting it. If the tassel does not yet have the meiotic stage desired, the incision created to harvest a portion of the tassel can be taped shut and the plant can be checked at a later time. As the plant continues to grow, new incisions may need to be made to access the tassel. The tassel will continue to mature until removed and fixed, and if only a portion of the tassel is removed, the remainder can generally continue to maturity and shed pollen. Portions of leaves 
and tassel may become tangled in the incision as the plant continues to grow and may need to be manually resolved to free the tassel for crossing.

\section{Additional Materials (also see Basic Protocol 2):}

- Plant with developing tassel

- 3:1 fixative: 3 parts $95 \%$ ethanol: 1 part $100 \%$ acetic acid

- Scalpel and forceps

\section{Locate tassel:}

1. Locate the developing tassel in the whorl of the plant (it should feel like a slightly spongy bulge).

2. Using a sharp scalpel, cut a slit vertically along the side of plant taking care not to slice into the tassel. Gently pull the two edges of the cut apart to expose the developing tassel.

3. Use forceps to pluck florets from the tassel. Try to grip the base of the spikelet pairs or the tip of the glume to avoid damaging the anthers.

4. Place florets in 3:1 fixative; keep on ice.

5. Perform Basic Protocol 2, steps 5 to 8 , with these not fully fixed anthers (it does not matter that they are not fully fixed when determining the stage of meiosis with acetocarmine).

If the anthers are found to be at the desired stage, use the two anthers identical to the one checked for chromosome preparation. One can dissect 
the florets in $70 \%$ ethanol, then place the two unused anthers back into 3:1 fixative in a 2-ml tube labeled with their stage of meiosis and fix $24 \mathrm{hr}$ at $4^{\circ} \mathrm{C}$. Afterwards, the anthers can be rinsed two times in $70 \%$ ethanol and used to prepare meiotic chromosomes by starting at Basic Protocol 2, step 10.

\section{Reagents and Solutions}

Use deionized, distilled water in all recipes and protocol steps.

\section{Acetocarmine stain}

For $100 \mathrm{ml}:$

- $45 \mathrm{ml}$ glacial acetic acid

- $55 \mathrm{ml}$ deionized water

- Heat on stir plate inside fume hood until boiling

- Add $0.5 \mathrm{~g}$ carmine and stir $20 \mathrm{~min}$ with continued heat

- Allow solution to cool, then filter to remove any precipitate

- Store for years at room temperature (if evaporation is prevented)

\section{Citric buffer, $1 x$}

Prepare a $5 \times$ working stock: 
- $50 \mathrm{mM}$ sodium citrate $50 \mathrm{mM}$ EDTA, adjusted to $\mathrm{pH} 5.5$ by adding citric acid

- Autoclave and store at room temperature

- Dilute to $1 \times$ with sterile water

- Use chilled on ice

\section{Digestive enzyme solution}

For $\sim 10 \mathrm{ml}$, mix thoroughly in tube or weigh boat on ice:

- $0.1 \mathrm{~g}(1 \% \mathrm{w} / \mathrm{w})$ Pectolyase Y-23 (Karlan Research Products)

- $0.2 \mathrm{~g} \mathrm{(2 \%} \mathrm{w/w)} \mathrm{Cellulase} \mathrm{Onozuka} \mathrm{R-10} \mathrm{(Karlan} \mathrm{Research} \mathrm{Products)}$

- $9.7 \mathrm{~g} 1 \times$ citric buffer (see recipe)

- Quickly dispense into 20-ml aliquots in thin-walled, 0.5-ml PCR tubes (ensure caps are closed tightly)

- Quick freeze on dry ice

- Store $\sim 1$ year at $-20^{\circ} \mathrm{C}$

\section{Commentary}

\section{Background Information:}

Meiotic and mitotic fixation of chromosomes is a useful tool in determining structure and function of genetic material in a cellular environment. In maize, the fixation process is accomplished through acetic acid treatment of developing 
tassel or root tissues. Utilization of these techniques has enabled maize researchers to determine many aspects of chromosome biology. If coupled with the more recent fluorescent in situ hybridization technology, higher resolution can be accomplished though the detection of specific nucleic acids with DNA or RNA probes.

\section{Critical Parameters and Troubleshooting:}

To obtain high-quality spreads, a few parameters need to be taken into consideration before beginning preparation of mitotic tissues. When choosing which roots to harvest, primary roots are generally the best. Longer treatment of roots in $\mathrm{N}_{2} \mathrm{O}$ gas results in more condensed chromosomes, while less time results in less-condensed chromosomes. Consistency in size of root tip slice is essential to keeping digestion times the same across samples. Placing a ruler by the root for measuring how much to slice results in more consistent root-tip lengths. Digestion time for root tips will vary depending on the length of the root tip as well as its diameter.

\section{Anticipated Results}

\section{Metaphase chromosome preparation:}

Resulting slides should have visible nuclei with visible chromosomes. At $600 \times$ magnification using a compound microscope, approximately three spreads 
can be found per field of view in a good preparation. The chromosome spreads should be relatively free of cytoplasm and other cellular matter, as well as having condensed chromatin so that individual chromosomes are easily identified (Fig. 1).

\section{Meiotic chromosome preparation:}

Depending on the stage of meiosis, the qualities of finished slides will vary. Finished slides of any stage of meiosis before Telophase I can be relatively cytoplasm free and cell walls of meiotic pollen mother cells should not be present. Cell preparations after telophase I will have an internal cell wall between the sister cells as well as some associated cytoplasm that the digestive enzyme cannot remove without over-digesting the cells. The chromosomes or chromatin of the sister cells should be mostly free of cytoplasm but will still have a visible barrier of cell wall and cytoplasm between them.

\section{Time Considerations}

\section{Preparation of metaphase chromosomes:}




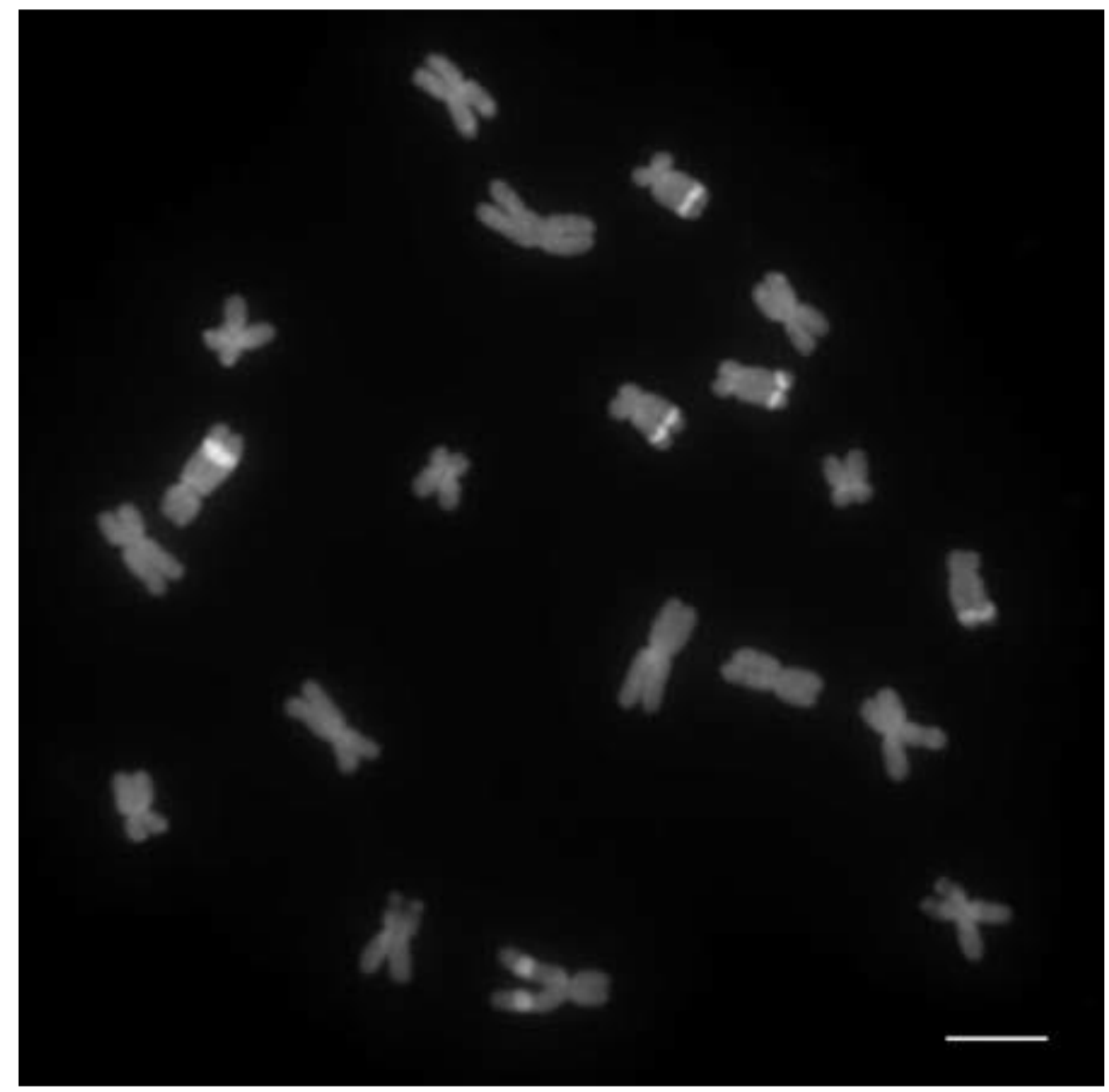

Figure 1 - Metaphase chromosome spread: A metaphase chromosome spread stained with DAPI (4',6-diamidino-2phenylindole) shown in greyscale. The scale bar in the lower right is $5 \mu \mathrm{m}$. Photo credit: Patrice S. Albert. 
Basic Protocol 1, steps 2 to 5 will require $4 \mathrm{hr}$. Roots can be stored in $70 \%$ ethanol up to 1 year at $20^{\circ} \mathrm{C}$. Basic Protocol 1 , steps 6 through 17 takes $3 \mathrm{hr}$. In general, 10 to 20 roots are processed at one time, and can generally be processed in $3 \mathrm{hr}$. Slides can be stored for several months at $4^{\circ} \mathrm{C}$, although if being used for FISH painting, better signal is found with fresh slides.

\section{Maize male meiosis chromosome preparation:}

Basic Protocol 2, steps 1 and 2 requires 1 day. The tassel can be stored up to 1 year at $-20^{\circ} \mathrm{C}$. Steps 3 to 9 take $15 \mathrm{~min}$ to $3 \mathrm{hr}$. The time required to find anthers at the appropriate stage is dependent on the skill level of the researcher. Steps 10 to 14 require $1.5 \mathrm{hr}$. Anthers can be stored overnight in $70 \%$ ethanol at $-20^{\circ} \mathrm{C}$. Steps 15 to 19 take $1 \mathrm{hr}$. Slides can be stored for months at $4^{\circ} \mathrm{C}$; however, better FISH signal is found with fresh slides.

\section{Acknowledgements}

Research on this topic in the Birchler laboratory is supported by NSF grants IOS- 1339198 and IOS-1444514. 


\section{References}

Kato A., (1999) Air drying method using nitrous oxide for chromosome counting in maize. Biotech. Histochem. 74:160-166.

McCaw M., Graham N., Cody J., Swyers N., Zhao C., and Birchler J. (2016) Fluorescence in situ hybridization to maize (Zea mays) chromosomes. Curr. Protoc. Plant Biol. 1:529-544.

Neuffer G.M., Coe E.H., and Wessler S.R. (1997) Mutants of Maize. Cold Spring Harbor Laboratories Press, Plainview, N.Y. 


\section{Appendix 4: Fluorescent In Situ Hybridization of Maize (Zea mays) Chromosomes}

Note: The information in this chapter was published under the title:

McCaw M.E., Graham N.D., Cody J.P., Swyers N.C., Zhao C., Birchler J.A.

(2016) Fluorescent in situ hybridization to maize (Zea mays) chromosomes. Curr.

Protoc. Plant Biol. 1:530-545. doi: 10.1002/cppb.20033 


\section{Summary}

Fluorescence In Situ Hybridization (FISH) is the annealing of fluorescent DNA probes to their complementary sequences on prepared chromosomes and subsequent visualization with a fluorescent microscope. In maize, FISH is useful for distinguishing each of the ten chromosomes in different accessions (karyotyping), roughly mapping single genes, transposable elements, transgene insertions, and identifying various chromosomal alterations. FISH can also be used to distinguish chromosomes between different Zea species in interspecific hybrids by use of retroelement painting.

\section{Introduction}

This protocol is an accompaniment to Preparation of Chromosomes from Zea mays (McCaw et al., 2016), which describes how maize chromosomes are isolated from cells and cross-linked to a glass slide. This protocol involves the preparation of DNA from a plasmid, the incorporation of fluorescent nucleotides into that DNA, and then the hybridization of that DNA to the prepared chromosomes.

\section{Target Selection and DNA Preparation}


FISH probes can be prepared for a wide variety of sequences, allowing for the technique to be used for many applications. However, it is important that the target is chosen extremely carefully before the procedure is begun. Many of the commonly used large target probes, with a total size $>4 \mathrm{~kb}$, consist of high copy number repeat sequences such as knob heterochromatin or centromeric CentC. For these sequences, fluorescent oligo probes can often be purchased that require little processing and are often more cost effective than the following methods. For small targets and single genes (targets as small as $1.4 \mathrm{~kb}$ have been identified using the Texas red flourochrome in our laboratory), it is suggested that the sequence be analyzed before being used for a probe. These probes for small targets must be processed differently than larger probes and the procedure is more time consuming. In addition, the signal is often weaker and harder to identify when compared to the background. As such, it is important to choose a sequence target that will give the largest chance of success. As a result, it is suggested that a sequence target be chosen that is larger than the minimum target size possible. In our experience, choosing a target greater than $3.5 \mathrm{~kb}$ for red probes and $6 \mathrm{~kb}$ for green probes produces the best images. When designing a small target probe, it must be ensured that the probe sequence is unique to the target location. Tools such as RepeatMasker (repeatmasker.org) should be utilized to ensure that any undesired repeats are removed from the probe sequence so the probe will not bind to any other homologous sites in the genome. If necessary, noncontiguous sequences can be used and pooled into a 
single probe and still appear as a single signal provided they are within 1 to $3 \mathrm{Mb}$ of each other.

The following protocol assumes that the DNA sequences have already been chosen and cloned into a plasmid. It is possible to use noncloned sequences in much the same way; however, it is suggested that these products are first gel purified if amplified from genomic DNA. As FISH probes can sometimes require over $100 \mathrm{ng}$ of DNA be added to each slide, it is suggested that at least $10 \mu \mathrm{g}$ of DNA be amplified for processing into a probe for hybridization.

\section{Materials:}

- Forward and reverse primers

- Target plasmids: many plasmids contain common primer binding sites, which can be used to amplify inserted DNA; if a unique primer is desired, it is suggested that a program such as Primer3 (http://bioinfo.ut.ee/primer3-

0.4.0/) be used to ensure proper primer design

- JumpStart REDTaq ReadyMix Reaction Mix (Sigma-Aldrich, cat. no. P0982)

- Deionized, distilled (dd) $\mathrm{H}_{2} \mathrm{O}$

- $3 \mathrm{M}$ sodium acetate $(\mathrm{pH} 5.2)$

- $100 \%$ ethanol

- $70 \%$ ethanol

- $1 \times$ TE or nuclease-free water 
- $1 \times$ TAE electrophoresis buffer

- $\quad$ Agarose (Sigma-Aldrich, cat. no. A9539)

- DNA Gel Loading Dye (6×) (Life Technologies, cat. no. R0611)

- GeneRuler 1-kb DNA Ladder (Life Technologies, cat. no. SM0311)

- Ethidium bromide (Sigma-Aldrich, cat. no. E7637)

- Target DNA cloned into a plasmid

- $\quad 0.6-\mu l$ PCR tubes

- Pipets

- Microcentrifuge

- Thermal cycler

- $\quad 1.7-\mathrm{ml}$ microcentrifuge tubes

- Vortex mixer

- Nanodrop spectrophotometer

- Gel electrophoresis equipment

- UV transilluminator

\section{DNA amplification:}

1. Assemble four of the following reactions (see Table 1) in separate $0.6-\mu l$ PCR tubes (may be assembled at room temperature).

While one large reaction can be used, we have found a larger yield can be obtained from four smaller reactions.

2. Mix gently by pipetting and spin down the reaction in a microcentrifuge. 
3. Use the following PCR conditions to amplify DNA. See Table 2 for PCR reaction conditions.

\section{DNA precipitation:}

4. Combine PCR reactions into a single $1.7-\mathrm{ml}$ tube.

5. Add $40 \mu \mathrm{l}$ of $3 \mathrm{M}$ sodium acetate (pH 5.2) and $1100 \mu \mathrm{l}$ of $100 \%$ ethanol.

6. Vortex thoroughly for $2 \mathrm{sec}$.

7. Incubate for $30 \mathrm{~min}$ to overnight at $-20^{\circ} \mathrm{C}$.

8. Centrifuge for $30 \mathrm{~min}$ at $16,000 \times \mathrm{g}, 25^{\circ} \mathrm{C}$.

9. Carefully discard the supernatant.

10. Add $1 \mathrm{ml}$ of $70 \%$ ethanol.

11. Centrifuge for $3 \mathrm{~min}$ at $16,000 \times \mathrm{g}, 25^{\circ} \mathrm{C}$.

12. Carefully discard supernatant and repeat steps 10 and 11 . 
Jumpstart RedTaq ReadyMix

$50 \mu 1$

Primer $1(100 \mu \mathrm{M})$

$0.5 \mu \mathrm{l}$

Primer $2(100 \mu \mathrm{M})$

$0.5 \mu 1$

Target Plasmid (50 ng)

$1 \mu 1$

$\mathrm{ddH}_{2} \mathrm{O}$

$48 \mu 1$

\section{Table 1 - PCR Reaction Mixture}




\begin{tabular}{lll}
\hline 1. & $94^{\circ} \mathrm{C}$ (initial denaturation) & $5 \mathrm{~min}$ \\
2. & $94^{\circ} \mathrm{C}$ (denaturation) & $30 \mathrm{Sec}$ \\
3. & $58^{\circ} \mathrm{C}$ (dependent on primer melting temperature; annealing) & $30 \mathrm{Sec}$ \\
4. & $72^{\circ} \mathrm{C}$ (extension) & $1 \mathrm{Min} / \mathrm{kb}$ of product \\
Repeat steps $2-4 \times 35$ & \\
5. & $72^{\circ} \mathrm{C}$ (final extension) & $5 \mathrm{Min}$ \\
6. & $4^{\circ} \mathrm{C}$ (hold) & Indefinite \\
\hline
\end{tabular}

Table 2 - PCR Reaction Conditions 
13. Completely remove the supernatant with a pipet.

14. Allow the pellet to dry 2 to $3 \mathrm{~min}$.

It is not necessary to completely dry the pellet, and overly dry pellets are difficult to redissolve.

15. Resuspend the pellet in 50 to $200 \mu \mathrm{l} 1 \times$ TE or nuclease-free water.

16. Once completely resuspended, measure concentration with a Nanodrop spectrophotometer.

\section{Check DNA integrity:}

17. Pour a $1 \%(w / v)$ agarose gel in $1 \times$ TAE.

18. Load $5 \mu \mathrm{l}$ of PCR product mixed with $1 \mu \mathrm{l}$ of $6 \times$ loading dye next to a lane containing $6 \mu$ of premixed GeneRuler 1-kb DNA Ladder.

19. Run the gel at $100 \mathrm{~V}$ for $30 \mathrm{~min}$, or until marker dye has traversed most of the gel.

20. Shake the gel for 15 to $30 \mathrm{~min}$ in $0.5 \mu \mathrm{g} / \mathrm{ml}$ ethidium bromide.

21. Visualize with a UV transilluminator.

22. Check integrity of DNA.

PCR product should be of the correct size and similar in concentration to that estimated by a Nanodrop or similar spectrophotometer. The PCR product should be visible as a single band, and free of any background in the lane. If PCR product is not as expected, consider adjusting primer sequences or annealing temperature. 


\section{Labeling Probe DNA by Nick Translation}

Fluorescent probes for the FISH procedure are generated through a nick translation reaction of purified PCR products or plasmid DNA. In this reaction, the enzymatic activity of two specialized proteins, DNase and DNA polymerase I, are utilized to incorporate fluorochrome-labeled dNTPs into template DNA. DNase enzymes randomly cleave the phosphodiester bonds in the DNA backbone, creating several free 5'-phosphoryl and 3'-hydroxyl ends. In these positions, DNA polymerase I performs both 5'-3' exonuclease and polymerase activity to remove nucleotides from the DNA backbone and replace them with free dNTPs from solution, respectively. Only a percentage of the incorporated dNTPs are labeled with specific fluorochromes.

Components of the nick translation reaction can be modulated to fit the requirements for specific targets. These requirements are determined by target size, which is defined by probe length and copy number on the chromosome. The length of the probe should be approximately 50 to $300 \mathrm{bp}$ after the nick translation reaction. To ensure this parameter is met, the DNase concentration can be decreased or increased for small and large templates, respectively. If copy number on the chromosome is low (these targets are usually transgenes or single-copy endogenous genes), a brighter probe will be desired for detection. To do this, DNA polymerase concentration can be increased to incorporate greater amounts of the fluorescent probe into the template DNA. 
The specific fluorochromes used in the nick translation reaction are also variable. Commonly used markers include Texas Red (red), fluorescein or Alexa Fluor 488 (green), coumarin (blue), and cyanine 5 (far red). For small targets, the use of Texas Red (red) is recommended for its increased brightness. Texas Red can also be used with medium or large targets. Alexa Fluor 488 (green) can be used for all targets, while coumarin (blue) and cyanine 5 (far red) should only be used for large targets. It should be noted that all fluorochromes are light sensitive and should be stored in a dark environment to maintain quality.

\section{Materials:}

- Ice

- Template DNA (PCR product or plasmid)

- $10 \times$ nick translation buffer (see recipe)

- Labeled dNTP mixture (1 nM)

- Nonlabeled dNTPs (2 mM each/ mixed)

- DNA polymerase I

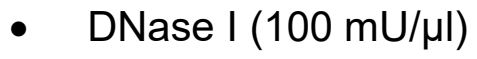

- 1× TE-saturated Bio-Gel P-60 (see recipe)

- $1 \times$ TE (see recipe)

- Autoclaved salmon sperm (10 mg/ml; see recipe)

- $3 \mathrm{M}$ sodium acetate $(\mathrm{pH}=5.2$; see recipe)

- $100 \%$ ethanol 
- $70 \%$ ethanol

- $2 \times \operatorname{SSC}$

- Thermal cycler or a lidded Styrofoam container filled with cold water

- Silane-treated glass wool

- Forceps

- Pasteur pipets

- Inoculating loops

- 1.6-ml collection tubes

- Vortex mixer

- Microcentrifuge $(>16,000 \times \mathrm{g} \max )$

- $\quad$ Lint-free tissues

- Heat block or water bath set to $65^{\circ} \mathrm{C}$

Nick translation:

For small targets (less than $30 \mathrm{~kb}$ ):

1a. Assemble the following reagents in a thermal cycler tube on ice:

$10 \mu \mathrm{l}$ template DNA ( $2 \mu \mathrm{g} ; 200 \mathrm{ng} / \mathrm{nl})$

$2 \mu \mathrm{l}$ of $10 \times$ nick translation buffer

$0.5 \mu$ labeled dNTPS ( $1 \mathrm{mM}$ )

$2 \mu \mathrm{l}$ nonlabeled dNTP (2 mM each, mixed)

Total volume: $14.5 \mu \mathrm{l}$. 
2a. Upon addition of each of the following enzymes to the reaction, mix the solution thoroughly by pipetting.

$8 \mu \mathrm{l} \mathrm{DNA}$ polymerase I $(10 \mathrm{U} / \mu \mathrm{l})$

$0.4 \mu \mathrm{l}$ DNase $(100 \mathrm{mU} / \mu \mathrm{l})$

Total volume: $22.9 \mu \mathrm{l}$

3a. Incubate the reaction mixture for $2 \mathrm{hr}$ at $15^{\circ} \mathrm{C}$ in a thermal cycler or water bath in a Styrofoam container. Overnight reactions can be carried out in a thermal cycler, but hold the temperature at $1^{\circ}$ to $4^{\circ} \mathrm{C}$ after the $2 \mathrm{hr}$ time mark.

It is optional to use $2 \mu$ stop buffer (EDTA $p H=8.0$ ) to terminate the reaction.

Optional stopping point: Store overnight at $-20^{\circ} \mathrm{C}$ in the dark.

If probe contains Texas Red (red) or cyanine 5 (far red)-labeled sequences, move to column purification. Our laboratory has found that this will produce a cleaner probe mixture, which reduces possible nonspecific binding. Probes containing fluorescein or Alexa Fluor 488 (green) or coumarin (blue) may proceed to ethanol precipitation of small target steps. Purification of these probes will negligibly affect binding efficiency.

\section{Procedure for large targets (larger than $100 \mathrm{~kb}$ ):}

1b. Assemble the following reagents on ice:

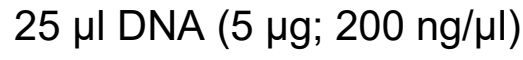

$5 \mu \mathrm{l}$ of $10 \times$ nick translation buffer 
$1 \mu$ l labeled dNTPs $(1 \mathrm{mM})$

$5 \mu \mathrm{l}$ nonlabeled dNTP

$7 \mu$ sterile $\mathrm{ddH} 2 \mathrm{O}$

Total volume: $43 \mu \mathrm{l}$.

$2 b$. Upon addition of each of the following enzymes to the reaction, mix the solution thoroughly by pipetting.

$6.25 \mu \mathrm{l}$ DNA polymerase I (10 U/ $\mathrm{\mu l})$

$1 \mu \mathrm{l}$ DNase $(100 \mathrm{mU} / \mu \mathrm{l})$

Total volume: $50.25 \mu \mathrm{l}$.

3b. Incubate the reaction mixture for $2 \mathrm{hr}$ at $15^{\circ} \mathrm{C}$ in a thermal cycler or water bath in a Styrofoam container. Overnight reactions can be carried out in a thermal cycler, but hold the temperature at $1^{\circ}$ to $4^{\circ} \mathrm{C}$ after the $2 \mathrm{hr}$ time mark.

It is optional to use $2 \mu \mathrm{l}$ of stop buffer (EDTA $p H=8.0)$ to stop the reaction.

Optional stopping point: Store overnight at $-20^{\circ} \mathrm{C}$ in the dark.

If probe contains Texas Red (red) or cyanine-5 (far red) labeled sequences, move to column purification. All other probe sequences may proceed to ethanol precipitation of large target steps.

\section{Probe purification:}

If the generated probe contains Texas Red (red) or cyanine-5 (far red) labeled sequences, the probe must be column purified. Probes containing 
fluorescein or Alexa Fluor 488 (green) or coumarin (blue) may proceed to ethanol precipitation steps.

\section{Column assembly:}

4. Using forceps, insert a piece of silane-treated glass wool into a glass Pasteur pipet.

5. With an inoculating loop, push the glass wool towards the tip of the Pasteur pipet.

6. Holding the pipet at an angle, fill with $1 \times$ TE-saturated Bio-Gel P-60 and place into a 1.6-ml collection tube. As the beads settle, TE will flow from the column. Replace the displaced TE with more TE-saturated Bio-Gel P-60 until beads are level with the constriction in the pipet.

7. Ensure the quality of the column by washing with $500 \mu \mathrm{lTE}$. Allow all TE to run through the column into the collection tube before using in probe purification.

\section{Column purification:}

All fluorochromes used to generate probes are light sensitive, so it is important to reduce light exposure when following the column purification steps below. It is suggested to carry out the experiment in a dimly lit room or place the column under a cardboard box after adding each TE volume.

8. Add probe mixture to the prepared column. 
9. Successively add the following amounts of TE to the column. Allow the designated volume to elute before moving to the next amount.

Amounts Directions

$50 \mu \mathrm{l}$ of $1 \times \mathrm{TE}$

Discard eluate.

$350 \mu$ of $1 \times$ TE

Discard eluate. Move column to new $1.6 \mathrm{ml}$ tube.

$350 \mu$ of $1 \times \mathrm{TE}$

Save eluate. Move column to new $1.6 \mathrm{ml}$ tube.

$350 \mu$ of $1 \times$ TE

Save eluate. Move column to new $1.6 \mathrm{ml}$ tube. Large

Probes: $350 \mu$ l of $1 \times$ TE Save eluate.

For small probes, two tubes should be recovered from column

purification. For large probes, three tubes should be recovered.

All purified probes may proceed to ethanol precipitation steps.

\section{Ethanol precipitation:}

For small targets (less than $30 \mathrm{~kb}$ ):

10a. Add the following components to each probe sample:

$3 \mu \mathrm{l}$ salmon sperm $(10 \mathrm{mg} / \mathrm{ml})$

$0.1 \mathrm{vol}(35 \mu \mathrm{l})$ of $3 \mathrm{M}$ sodium acetate $(\mathrm{pH} 5.2)$

$2.5 \mathrm{vol}(875 \mu \mathrm{l})$ of $100 \%$ ethanol

11a. Vortex for $2 \mathrm{sec}$ to mix components.

12a. Incubate for $+2 \mathrm{hr}$ at $-20^{\circ} \mathrm{C}$.

Samples may be stored overnight at $-20^{\circ} \mathrm{C}$.

13a. Centrifuge in a microcentrifuge for $30 \mathrm{~min}$ at $16,000 \times \mathrm{g}, 25^{\circ} \mathrm{C}$.

14a. Carefully pour off supernatant so as not to disturb pellet. 
15a. Rinse the pellet with $500 \mu \mathrm{l}$ of $70 \%$ ethanol. Repeat rinse.

16a. Carefully pour off ethanol without disturbing the pellet, then invert the tube and blot the rim on a lint-free tissue to remove as much ethanol as possible.

Brief centrifugation for $1 \mathrm{~min}$ at $16,000 \times \mathrm{g}, 25^{\circ} \mathrm{C}$, before decanting may help secure the pellet.

17a. Add $10 \mu \mathrm{l}$ of $2 \times \mathrm{SSC} / \mathrm{TE}$ mixture to tubes.

18a. Incubate on heat block or in water bath set at $65^{\circ} \mathrm{C}$ to dissolve pellet. Vortexing may also help with this process.

19a. Store clean probes up to 5 years at $-20^{\circ} \mathrm{C}$. For large targets (less than $30 \mathrm{~kb}$ )

\section{Procedure for large targets (larger than $100 \mathrm{~kb}$ ):}

10b. Add the following components to each probe sample:

$5 \mu \mathrm{l}$ salmon sperm $(10 \mathrm{mg} / \mathrm{ml})$

$0.1 \mathrm{vol}$ of $3 \mathrm{M}$ sodium acetate $(\mathrm{pH} 5.2)$

$2.5 \mathrm{vol}$ of $100 \%$ ethanol.

11b. Vortex for $2 \mathrm{sec}$ to mix the components.

12b. Incubate for $+2 \mathrm{hr}$ at $-20^{\circ} \mathrm{C}$.

Samples may be stored overnight at $-20^{\circ} \mathrm{C}$. 
13b. Centrifuge in a microcentrifuge for $30 \mathrm{~min}$ at $16,000 \times \mathrm{g}, 25^{\circ} \mathrm{C}$.

14b. Carefully pour off supernatant to not disturb pellet.

15b. Rinse pellet with $500 \mu \mathrm{l} 70 \%$ ethanol. Repeat rinse.

16b. Carefully pour off ethanol without disturbing the pellet, then invert the tube and blot the rim on a lint-free tissue to remove as much ethanol as possible.

Brief centrifugation for $1 \mathrm{~min}$ at $16,000 \times \mathrm{g}, 25^{\circ} \mathrm{C}$, before decanting may help secure the pellet.

17b. Add $10 \mu$ of $2 \times$ SSC/TE mixture to tubes.

18b. Incubate on heat block or in water bath set to $65^{\circ} \mathrm{C}$ to dissolve pellet. Vortexing may also help with this process.

19b. Store the clean probes up to 5 years at $-20^{\circ} \mathrm{C}$.

\section{Fluorescence In Situ Hybridization}

Probes produced in the previous sections will nowbe combined and added to the prepared slides from the previous protocol. Once the probe is added to the slide both the probe DNA and chromosomes are subjected to temperatures just below boiling to form single stranded DNA. The slides are then incubated at $55^{\circ} \mathrm{C}$ to allow the DNA to reanneal, thus hybridizing the fluorescent probes to the chromosomes. Slides are then finished by removing the temporary 
coverslip, adding a mounting medium, and affixing an optically clear glass coverslip.

\section{The Karyotyping Cocktail:}

To distinguish chromosomes from each other, a "Karyotyping cocktail" has been developed. This cocktail uses blue, green, red, and far red (pseudo-colored as white during image processing) probes to label large chromosomal targets. In addition to the basic colors, teal, orange, and yellow are also produced by labeling a target with two different colors in the correct proportions. Table 3 provides the common sequences used for karyotyping and their relevant vector information. Table 4 lists the primer sequences. Table 5 provides the standardized colors of each target and the concentrations in which they are commonly used. Any sequences labeled in both green and red should appear yellow or orange once the color channels are merged. Similarly, any sequences labeled with both blue and green will appear as some variation of teal once merged. The concentrations provided in Table 5 should be considered a starting point, and the relative concentrations of each probe in the cocktail may need to be adjusted in order to produce the desired colors. Differences in exposure time and intensity when photographing each color channel may also result in colors which do not match the expected colors. Changing the intensity of channels during image processing can also correct the merged colors. More detailed information on image processing is outlined in the supplementary YouTube video. 


\begin{tabular}{|c|c|c|c|c|c|}
\hline $\begin{array}{l}\text { Target (clone } \\
\text { name) }\end{array}$ & Vector & Bacteria & $\begin{array}{l}\text { Alternate } \\
\text { primers }\end{array}$ & $\begin{array}{l}\text { PCR product } \\
\text { size }\end{array}$ & $\begin{array}{l}\text { Genbank } \\
\text { accession no. }\end{array}$ \\
\hline Cent4 & pBluescript & $\mathrm{DH} 5 \alpha$ & & $800 \mathrm{bp}$ & AF242891 \\
\hline CentC & pBluescript & $\mathrm{DH} 5 \alpha$ & & $540 \mathrm{bp}$ & $\begin{array}{l}\text { AF078918 - } \\
\text { AF078923 }\end{array}$ \\
\hline TR-1 (M77) & pGem-T & DH5 $\alpha$ & MR77 F/R & $400 \mathrm{bp}$ & AF020266 \\
\hline $\begin{array}{l}\text { 5S Ribosomal } \\
\text { RNA (2-3-3) }\end{array}$ & pGem-T & DH5 $\alpha$ & BEH 2 F & $700 \mathrm{bp}$ & CL569181 \\
\hline NOR 173 & pBluescript & DH10B & & $2 \mathrm{~kb}$ & CL569243 \\
\hline Knob 3-Copy & pGem-T & Stbl4 & Knob F/R & $540 \mathrm{bp}$ & $\begin{array}{l}\text { M32521 - } \\
\text { M32532 }\end{array}$ \\
\hline TAG (1-26-2) & pGem-T & Stbl4 & EBH $1 \mathrm{~F}$ & 500 bp smear & \\
\hline $4-12-1$ & pGem-T & $\mathrm{DH} 5 \alpha$ & BEH 2 F & $1 \mathrm{~kb}$ & CL569186 \\
\hline $\begin{array}{l}1.1 \\
\text { (pMTY9ER) }\end{array}$ & pBluescript & DH5 $\alpha$ & M13 F/R & $1.1 \mathrm{~kb}$ & U39642 \\
\hline
\end{tabular}

Table 3 - Plasmids for amplifying maize probes. M13 primers can be used to amplify targets in all plasmids; however, the listed alternate primers have been used and found to give stronger signal. In the case of EBH and $\mathrm{BEH}$ primers, only forward primers are required as the reverse primer is the same sequence. Plasmids are available from the corresponding author. 


\begin{tabular}{ll} 
M13 Forward & CCC AGT CAC GAC GTT GTA AAA CG \\
M13 Reverse & AGT GGA TAA CAA TTT CAC ACA GG \\
EBH1 F & AGA ATT CGG ATC CAA GCT TCT GGT TTG \\
BEH2 F & AGG ATC CGA ATT CAA CGT TGT CTT TG \\
Knob F & GGC CAC ACA ACC CCC ATT TTT G \\
Knob R & GGC CAT TGA TCA TCG ACC AGA \\
MR77 F & CCT CAA ATG CCG TTT CCT AT \\
MR77 R & CAC TCA CGC AAT TTG GCT AA \\
\hline
\end{tabular}

${ }^{a}$ All primers are listed $5^{\prime}$ to $3^{\prime}$.

Table 4 - Primer Sequences for Amplifying Maize Probes. 


\begin{tabular}{|c|c|c|c|c|}
\hline Color & $\begin{array}{l}\text { Target sequence } \\
\text { (clone name) }\end{array}$ & $\begin{array}{l}\text { Stock } \\
\text { concentration }\end{array}$ & $\begin{array}{l}\text { Final } \\
\text { concentration }\end{array}$ & $\begin{array}{l}\text { Amount } \\
\text { per slide }\end{array}$ \\
\hline Blue & Knob & $200 \mathrm{ng} / \mu \mathrm{l}$ & $40 \mathrm{ng} / \mu \mathrm{l}$ & $1.0 \mu \mathrm{l}$ \\
\hline Green & NOR 173 & $10 \mathrm{ng} / \mu \mathrm{l}$ & $0.2 \mathrm{ng} / \mu \mathrm{l}$ & $0.1 \mu 1$ \\
\hline Green & CentC & $200 \mathrm{ng} / \mu \mathrm{l}$ & $2 \mathrm{ng} / \mu \mathrm{l}$ & $0.1 \mu \mathrm{l}$ \\
\hline Green & $\begin{array}{l}\text { 5S Ribosomal } \\
\text { RNA (2-3-3) }\end{array}$ & $200 \mathrm{ng} / \mu 1$ & $18 \mathrm{ng} / \mu \mathrm{l}$ & $0.45 \mu 1$ \\
\hline Green & $4-12-1$ & $200 \mathrm{ng} / \mu \mathrm{l}$ & $40 \mathrm{ng} / \mu \mathrm{l}$ & $1.0 \mu 1$ \\
\hline Red & TAG (1-26-2) & $200 \mathrm{ng} / \mu \mathrm{l}$ & $6 \mathrm{ng} / \mu 1$ & $0.15 \mu 1$ \\
\hline Red & Cent4 & $200 \mathrm{ng} / \mu \mathrm{l}$ & $10 \mathrm{ng} / \mu \mathrm{l}$ & $0.25 \mu 1$ \\
\hline Red & $\begin{array}{l}\text { 5S Ribosomal } \\
\text { RNA (2-3-3) }\end{array}$ & $200 \mathrm{ng} / \mu \mathrm{l}$ & $18 \mathrm{ng} / \mu \mathrm{l}$ & $0.45 \mu 1$ \\
\hline Red & 1.1 (pMTY9ER) & $200 \mathrm{ng} / \mu \mathrm{l}$ & $40 \mathrm{ng} / \mu 1$ & $1.0 \mu \mathrm{l}$ \\
\hline Far Red & TR-1 (M77) & $200 \mathrm{ng} / \mu \mathrm{l}$ & $20 \mathrm{ng} / \mu \mathrm{l}$ & $0.5 \mu \mathrm{l}$ \\
\hline \multicolumn{5}{|l|}{ Optional: } \\
\hline Blue & NOR 173 & $200 \mathrm{ng} / \mu \mathrm{l}$ & $200 \mathrm{ng} / \mu \mathrm{l}$ & $0.74 \mu 1$ \\
\hline
\end{tabular}

Table 5 - A list of the primers used and what concentration to produce a karyotype similar to those described by Kato et al., 2004. Karyotypes of common inbred lines are available through the link in the Internet Resources section. Not using the optional blue NOR 173 will produce a karyotype that has a green NOR instead of teal like the karyotypes shown, but will not affect identification of chromosome 6 because the NOR is not likely to be confused with any other signal. 
Maize inbred lines are highly variable. Most of the probes in this cocktail target repetitive sequences, which will vary in size and location among different inbred lines (Albert et al., 2010).

Along with chromosome size and shape, several sequences frequently appear in the same locations and can aid in chromosome identification in a line for which a karyotype is not readily available. The signal color and location, and relative chromosome shape are shown in Figure 1. These sequences will not always be present, and their copy number and thus signal strength may vary. A Knob near the distal tip of $9 S$ is present in all analyzed maize lines but may not be detectable in roughly $50 \%$ of karyotyped lines due to low copy number and the presence of much larger Knobs on other chromosomes with stronger signal. The presence of a Knob at the distal tip of $10 \mathrm{~S}$ has not been reported (there is a pericentromeric knob on $10 \mathrm{~S}$ in the line OH7B.)

Using these amounts of each probe should result in a final volume of $5 \mu \mathrm{l}$ of probe per slide. $3-5 \mu$ additional $2 \times$ SSC $1 \times$ TE can be added to the probe to raise the final volume to $8-10 \mu \mathrm{l}$ per slide, which helps prevent bubbles of air between the coverslip and slide. These probe concentrations serve as a starting point.

\section{Basic Green Probe Cocktail with Single Gene Target Red:}

When attempting to localize a single gene such as a transgene insertion, a cocktail of a few distinctive chromosomal markers labeled in green, along with 


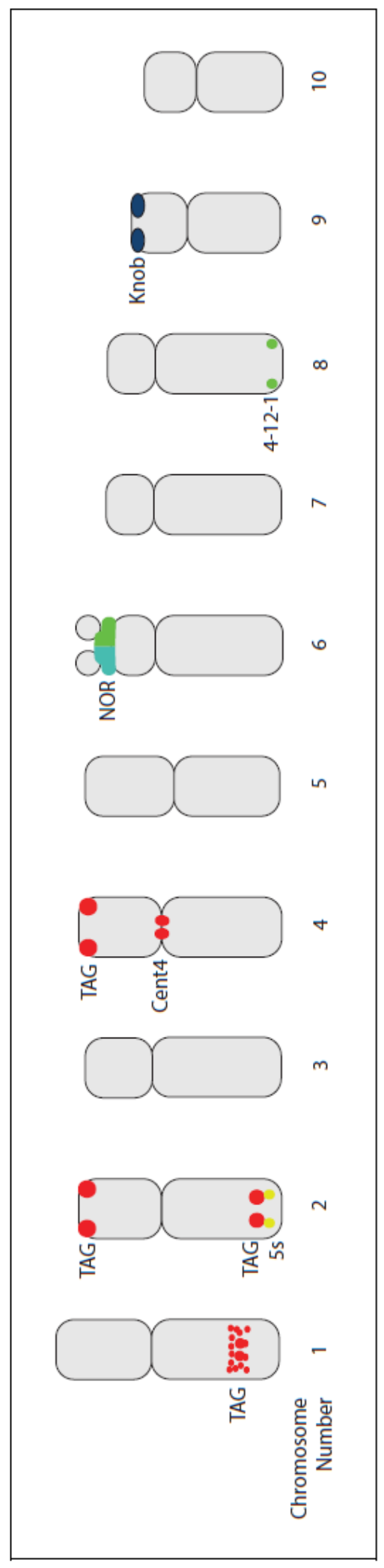

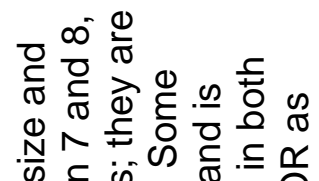

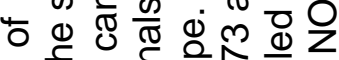

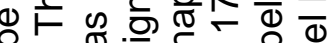

ब.

w.

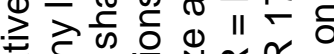

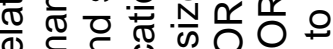

Ф ह ธ

(1) क 0 응

인

ডర

등 휴

它

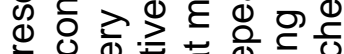

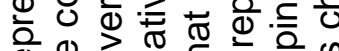

ब 0 ब $<0$ 은

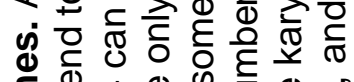

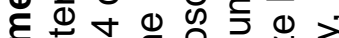

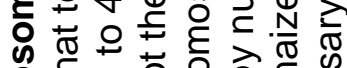

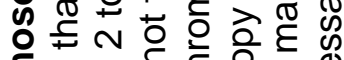
ह

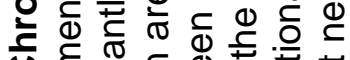

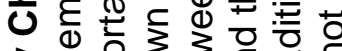

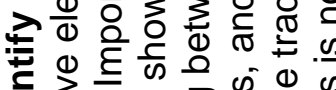
守 응 峁

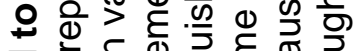

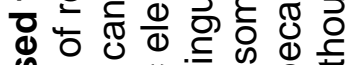
为

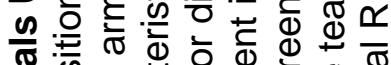
তั

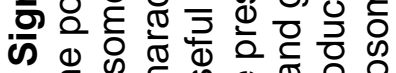

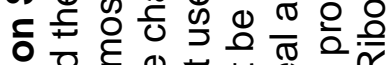

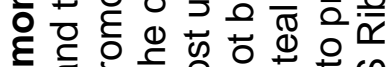

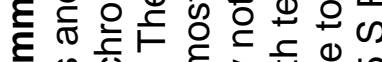

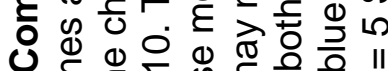

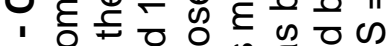

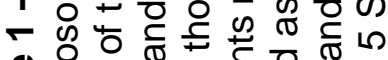
을 ब

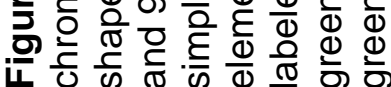


chromosome morphology is generally enough to identify most chromosomes in most lines. Figure 2 provides a representation of the basic size and shape of the chromosomes of the transformable lines Hill A and B. Other lines can be compared to the karyotypes provided in the link in the Internet Resources section. The copy number of CentC, and thus intensity of signal, is an important characteristic for differentiating between similarly sized chromosomes like chromosomes 7 and 8 of Hill.

The basic green cocktail is CentC, TAG, and NOR 173 diluted in $2 \times$ SSC $1 \times$ TE for a volume of 8-10 $\mu \mathrm{l}$ per slide. It is easier to use 6-FAM labeled oligo for CentC (CCT AAA GTA GTG GAT TGG GCA TGT TCG) and TAG (simply called TAG because it is an oligo; $1-26-2$ is the name of the original plasmid clone) (AG[TAG]18) from Integrated DNA Technologies. The concentrations used for this cocktail are listed in Table 6. The single gene probe can then be labeled with red.

With oligo probes, $2 \times$ SSC $1 \times$ TE is used to dilute probes from stock concentrations of $500-200 \mathrm{ng} / \mu$ to less concentrated working stocks. Less concentrated working stocks are prepared to provide more accurate measurement of each probe and reduce the number of freeze-thaw cycles that the probe must endure.

$1.0 \mu \mathrm{l}$ of $200 \mathrm{ng} / \mu \mathrm{l}$ single gene probe in red should be sufficient to visualize even 2.0 to $2.5 \mathrm{~kb}$ targets. Once hybridization of the single gene probe is confirmed, it may be possible to reduce the concentration to optimize the amount of probe used while still maintaining visible hybridization. 


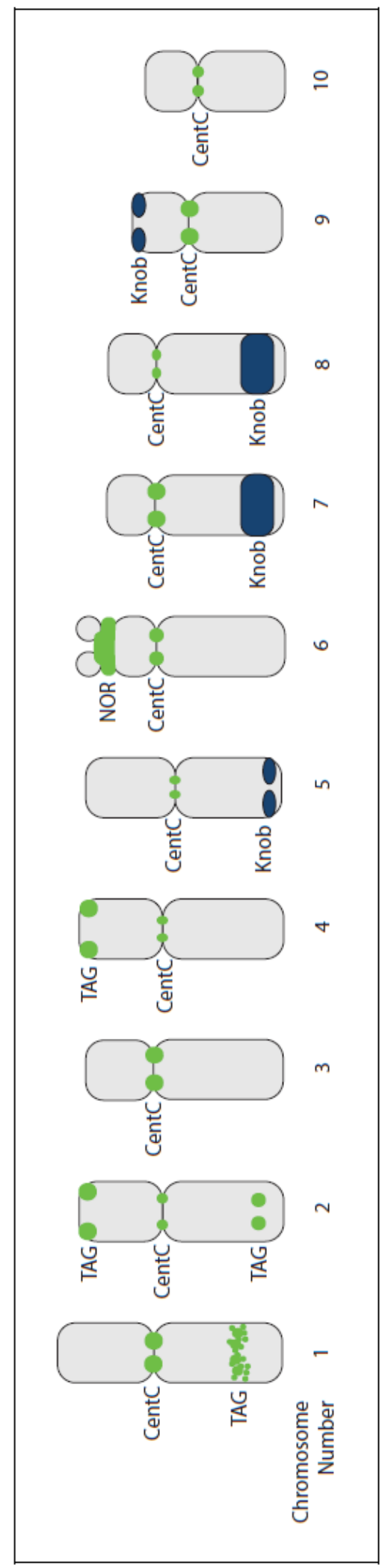

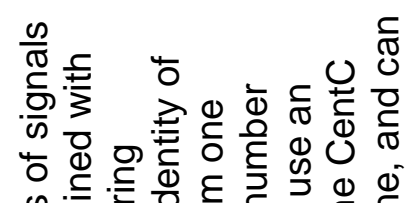

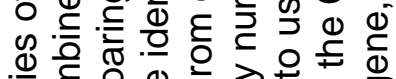

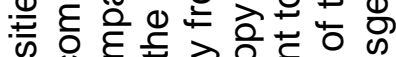
ब \pm . 정

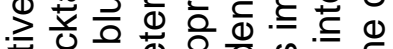
ণิ

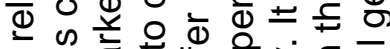

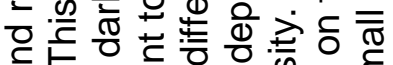
ฮั

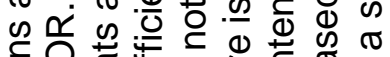

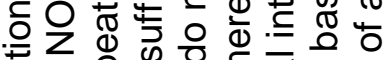

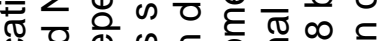

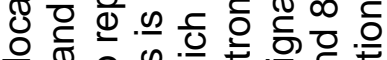

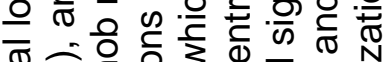

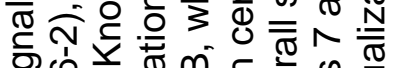

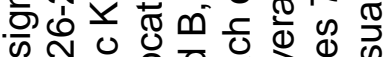
Ð “它 인

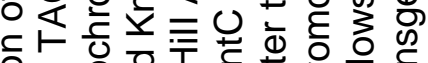
은

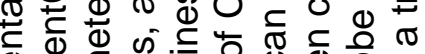
Ф

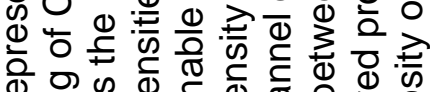
ब이 ब

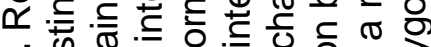

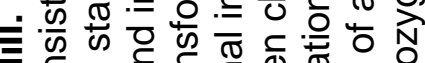

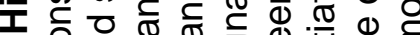

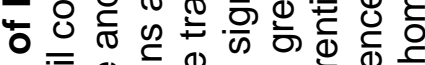
永

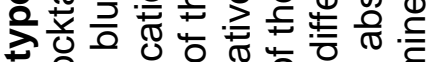
ㅇㅇㅇ응 웅 䒕 ส

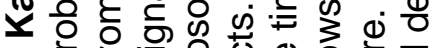

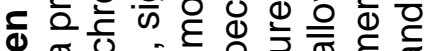

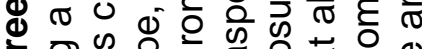

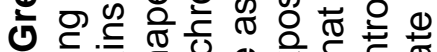

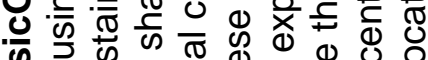
(

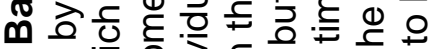

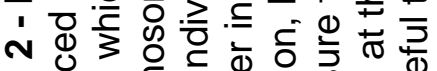

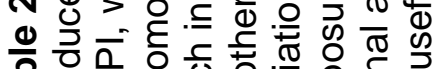

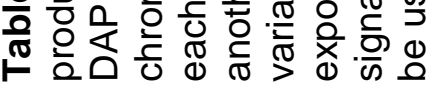




\begin{tabular}{lllll}
\hline Color & $\begin{array}{l}\text { Target } \\
\text { Sequence }\end{array}$ & Source & $\begin{array}{l}\text { Working/stock } \\
\text { Concentration }\end{array}$ & $\begin{array}{l}\text { Amount per } \\
\text { Slide }\end{array}$ \\
\hline Green & CentC & Oligo & $20 \mathrm{ng} / \mu 1$ & $1.5 \mu 1$ \\
Green & TAG & Oligo & $20 \mathrm{ng} / \mu 1$ & $0.8 \mu 1$ \\
Green & NOR 173 & $\begin{array}{l}\text { Nick } \\
\text { translated }\end{array}$ & $10 \mathrm{ng} / \mu 1$ & $0.3 \mu 1$ \\
& & PCR product & & \\
Red & Small target of & Nick & $200 \mathrm{ng} / \mu 1$ & $1 \mu 1$ \\
& interest & translated & & \\
& & PCR product & & \\
\hline
\end{tabular}

Table 6 - Basic Green Cocktail Targets and Concentrations. 
Because no probes labeled with blue are used in this cocktail, Vectashield with DAPI is added to these slides, see the Troubleshooting section in the Commentary for information about dilution.

\section{Materials:}

- Probe Prepared cells (see McCaw et al., 2016)

- $2 \times$ SSC: diluted from $20 \times$ SSC

- Vectashield (with or without DAPI)

- 22-mm $\times$ 22-mm plastic coverslips

- Square wooden dowel rods

- Metal tray

- $\quad$ Lint-free tissues

- Boiling water bath

- Airtight box Paper towels

- $55^{\circ} \mathrm{C}$ incubator

- Coplin jar

- $\quad 24-\mathrm{mm} \times 50-\mathrm{mm}$ glass coverslips

1. Add 8 to $10 \mu \mathrm{l}$ of probe to the center of the prepared cells (see McCaw et al., 2016) and cover with a 22-mm $\times 22-\mathrm{mm}$ plastic coverslip. If there are any air bubbles under the coverslip, try to press them out to the edge, but do not slide the coverslip. 
It may be helpful to place the slides on two square wooden dowels aligned parallel to each other about 3.0-cm apart with the cell spread centered between the dowels. This placement will facilitate centering the probe and coverslip and the height will allow the tips of your fingers to go past the slide.

2. Place the slides face up in a metal tray with three to six layers of wet (with water) lint-free tissue between the slides and the tray and then place the tray in a boiling water bath for 5 min.

If the water bath has a lid or foil over it, make certain to use a cover over the slides to prevent condensed water from dripping onto them; a $1+\mathrm{cm}$ deep pipet box lid works well for this purpose.

Tissues should be wet enough to transfer heat, but not wet enough to pool water over slides. Soaking the tissues in the tray thoroughly and holding the tray vertically until water stops pouring and only infrequently drips out should give approximately the proper moisture level.

3. After 5 min, move the slides to an airtight box with moistened paper towels or tissues for humidity. The slides should be elevated from the paper towels in a metal slide rack or on a pipet tip box lid. Firmly seal the box and place overnight at $55^{\circ} \mathrm{C}$. 
It has been found that this overnight hybridization is not necessary for abundant repeats probed with only end labeled oligos such as telomere or CentC. If the slide is being prepared with only oligo probes, step 3 can be skipped and one can proceed directly to step 4.

4. Submerge slides vertically into a Coplin jar filled with room temperature $2 \times$ SSC to remove coverslips.

The coverslips should fall off the slides almost immediately when placed in the $2 \times$ SSC. They will frequently fail to fall off if the slides cool too much before submersion.

5. Move slides to a second Coplin jar filled with $2 \times \mathrm{SSC}$ that is at $55^{\circ} \mathrm{C}$ and place in $55^{\circ} \mathrm{C}$ incubator for $35 \mathrm{~min}$ to remove unbound probe.

6. Remove the slide from $2 \times$ SSC and shake dry by hand. Wipe the back side of the slide dry with a lint-free tissue and place on parallel square dowels from step 1.

The position of the coverslip should be visible as a square where the $2 x$ SSC adheres more readily. Center this square between the dowels to accurately add Vectashield in next step.

7. Add a drop of Vectashield (with or without DAPI) to the center of the probed cells and place a $22-\mathrm{mm} \times 50-\mathrm{mm}$ glass coverslip on the slide. 
To reduce bubbles of air under the coverslip lower the coverslip down until it just touches the Vectashield then let it go gently. In addition, make certain the slide is almost completely dry before adding Vectashield and the coverslip or the extra $2 \times$ SSC will seal in air bubbles.

As the Vectashield spreads, the coverslip may shift off center. Keep the coverslip aligned as it adheres to the slide because shifting the coverslip later will smear the cells and ruin parts of the slide.

If Vectashield without DAPI is applied to the slides, searching for chromosome spreads under fluorescence is slightly more difficult. It is recommended to search for spreads on the green channel and look for clearly defined, paired dots of signal. Once zoomed in to 1000x, chromosomes can be visualized by their slight background illumination. When producing karyotypes without DAPI, it is useful to keep some level of background during image processing to allow visualization of chromosome shape.

The slide is now ready to image on a fluorescence microscope or can be stored up to 3 months in the dark at $4^{\circ} \mathrm{C}$ without appreciable loss of quality. Storage for periods of two years or more are possible but may result in reduced signal strength. 
After storage at $4^{\circ} \mathrm{C}$ it is recommended that slides be allowed to reach room temperature and ensure the slides are free from condensation before applying oil for visualization with a fluorescence microscope to avoid creating an emulsion of oil and water, which can blur images and requires cleaning the lenses of the microscope.

\section{Reagents and Solutions}

For culture recipes and steps, use sterile tissue-grade water. For other purposes, use deionized, distilled water or equivalent in recipes and protocol steps.

\section{Loading dye, 6x:}

For $10 \mathrm{ml}$ of solution, add $25 \mathrm{mg}$ of $0.25 \%$ bromphenol blue, $25 \mathrm{mg}$ of $0.25 \%$ xylene cyanol FF, and $4 \mathrm{~g}$ of $40 \%$ sucrose to TE buffer. Fill to $10 \mathrm{ml}$ with TE buffer. Store up to 5 years at $4^{\circ} \mathrm{C}$.

\section{Nick translation buffer, 10x stock:}

Dissolve $6.05 \mathrm{~g}$ Tris base $(500 \mathrm{mM})$ and $476.0 \mathrm{mg} \mathrm{MgCl} 2 .(50 \mathrm{mM})$ in water. Adjust $\mathrm{pH}$ to 7.8 with hydrochloric acid $(\mathrm{HCl})$. Add $701 \mu$ of 2mercaptoethanol $(100 \mathrm{mM})$ to water and bring volume to $100 \mathrm{ml}$. Autoclave to sterilize. Solution should be stored in $\sim 993-\mu l$ aliquots. Store aliquots up 
to 5 years at $-20^{\circ} \mathrm{C}$. Upon thawing, add $7 \mu$ of 2-mercaptoethanol. Replace stocks when odor is gone.

Addition of bovine serum albumin fraction V, Sigma-Aldrich A-9647 (+ 100 $\mu \mathrm{g} / \mathrm{ml}$ ) is optional.

\section{Salmon sperm DNA:}

Product used is Sigma-Aldrich D1626. Dissolve DNA in 1× TE buffer $(\mathrm{pH}=$ 7.8) to a concentration of $10 \mu \mathrm{g} / \mu \mathrm{l}$, shake or stir if necessary. Once completely dissolved, autoclave $30 \mathrm{~min}$. To determine the size of the DNA fragments, run the sample on an agarose gel. The fragments should be 100 to $300 \mathrm{bp}$. Store as $1-\mathrm{ml}$ aliquots up to $10+$ years at $-20^{\circ} \mathrm{C}$; a working stock can be left at room temperature for years.

\section{Sodium acetate, $3 \mathrm{M}$ :}

For $200 \mathrm{ml}$ of solution, add $49.2 \mathrm{~g}$ (anhydrous) of 3.0 Msodium acetate to a container and fill to $200 \mathrm{ml}$ with water. Add glacial acetic acid to solution until $\mathrm{pH}$ reaches 5.2. Autoclave to sterilize. Store up to 10 years at room temperature.

SSC, 20x: 
For 1 liter of $20 \times \mathrm{SSC}$, add $175.3 \mathrm{~g} \mathrm{NaCl}$ and $88.2 \mathrm{~g}$ sodium citrate in $800 \mathrm{ml}$ of water. Adjust the $\mathrm{pH}$ to 7.0 using $\mathrm{HCl}$. Bring volume to 1 liter and autoclave to sterilize. Store up to 10 years at room temperature.

SSC, $2 x / T E, 1 x:$

For $100 \mathrm{ml}$, dilute $10 \mathrm{ml} 20 \times \mathrm{SSC}$ and $10 \mathrm{ml} \mathrm{10 \times} \mathrm{TE} \mathrm{in} 80 \mathrm{ml}$ of autoclaved deionized water. Store in $50-\mathrm{ml}$ aliquots up to 5 years at room temperature.

\section{Stop buffer (EDTA):}

For $500 \mathrm{ml}$ of solution, add $93.1 \mathrm{~g}$ (dehydrate) of 0.5 Mdisodium EDTA to container and bring volume to $500 \mathrm{ml}$ with water. Adjust $\mathrm{pH}$ to 8.0 with $\mathrm{NaOH}$ ( 20 g). Autoclave to sterilize. Store up to 10 years at room temperature.

TAE, 50x:

Dissolve $242 \mathrm{~g}$ trizma base, $14.6 \mathrm{~g}$ EDTA, and $57.1 \mathrm{ml}$ of acetic acid in 500 $\mathrm{ml}$ of water and fill to $1000 \mathrm{ml}$. Store up to 5 years at room temperature. For a $1 \times$ stock, add 1 volumes of TAE (50x) to 49 volumes of water.

TE, 10x: 
For $1000 \mathrm{ml}$ of solution, add $12.1 \mathrm{~g}$ of $100 \mathrm{mM}$ Tris base and $3.7 \mathrm{~g}$ (dehydrate) of $10 \mathrm{mM}$ disodium EDTA to a container. Fill to $1000 \mathrm{ml}$ with water and adjust the $\mathrm{pH}$ to 7.6 using $\mathrm{HCl}$. Autoclave to sterilize. Store up to 10 years at room temperature.

TE saturated Bio-Gel P-60, 1× stock:

Product used is Bio-Rad 150-4160. Mix polyacrylamide beads and 1× TE, pH 7.6. Allow solution to sit at $4^{\circ} \mathrm{C}$ overnight before use. Stable up to 3 years at $4^{\circ} \mathrm{C}$.

\section{Vectashield and DAPI:}

Dilute Vectashield with DAPI in plain Vectashield. Proper dilution varies based on sensitivity of the microscope being used; see Troubleshooting for more details.

\section{Commentary}

\section{Background Information:}

FISH is a useful tool for the analysis and visualization of chromosomes. It has been used to identify single genes (J. C. Lamb et al., 2007), transgene insertions (Yu, Lamb et al., 2006; Gaeta et al., 2011), repetitive elements (Kato et 
al., 2004; Lamb et al., 2007), and transposable elements (Yu et al., 2007). FISH has also been used to study interspecies hybrids and allopolyploids by using retroelements to develop probes that paint genomes (Lamb and Birchler, 2006). This variety of uses has made FISH an important tool for understanding the makeup of the maize genome as well as in genetic engineering for the ability to locate transgene insertions easily.

FISH follows the basic idea of performing a Southern blot analysis in that it exploits the ability of single-stranded DNA to bind to its complement (Bishop, 2010). Desired cells are fixed to microscope slides and are hybridized to a nucleic acid probe, which anneals to its complementary sequence in the DNA of the specimen. This nucleic acid probe is labeled with a reporter molecule that is, in the case of the FISH protocol described here, a fluorochrome. This enables the detection of the probe, which is hybridized to the target DNA, by fluorescence microscopy, which visualizes the fluorescent signature of the probe (Bishop, 2010).

Using FISH as a tool has some disadvantages that are worth considering before beginning. The size of the probe target needs to be carefully considered as targets of smaller size than $2.5 \mathrm{~kb}$ are not easily detectible, though targets as small as $1.4 \mathrm{~kb}$ can be detected. Small targets that are not immediately adjacent but residing in proximity and adding to more than $3 \mathrm{~kb}$ can be detected well. Detection of duplications of probed DNA can be hard to visualize using FISH because of the limitations of fluorescence microscopes to identify individual probe binding sites in close proximity. Photobleaching of probes on slides is 
possible as well as nonspecific binding if probe sequences are not carefully considered. False-positives and false-negative results are possible because of the previously mentioned nonspecific binding of improperly designed probes, errors in processing of images, and imperfect hybridizations.

\section{Critical Parameters:}

Probe sequence must be carefully considered in regard to both size of sequence as well as its constitution. The target size is also important for deciding what fluorochrome with which the probe should be labeled.

\section{Troubleshooting:}

\section{DNA Preparation:}

Steps 1 to 3 : It is suggested that a small $(10 \mu \mathrm{l}) \mathrm{PCR}$ test reaction be carried out prior to performing the full PCR reaction and examined on an agarose gel if primers and plasmid DNA have not been used previously. Conditions, such as annealing temperature and cycle number, should be adjusted until PCR results in a single strong band before proceeding with large-scale PCR for probe DNA.

\section{Fluorescence In Situ Hybridization:}


Vectashield and DAPI: DAPI (4',6- diamidino-2-phenylindole) is a blue fluorescent dye, which stains chromatin. Vectashield is a mounting medium for fluorescence microscopy that is available in multiple formulations, notably with DAPI and without DAPI. For probe mixtures that do not use a blue probe DAPI is useful for distinguishing the chromosomes as a whole. Depending on the microscope, the concentration of DAPI in the Vectashield may need to be adjusted. For some microscopes the factory formulation of Vectashield with DAPI works well but with others it is too intense. Using a 1:10, 1:20, or 1:40 dilution of Vectashield with DAPI to standard Vectashieldmay be necessary. With lower concentrations of DAPI the more condensed regions of chromatin such as knobs can often be distinguished from less condensed chromatin and aid in distinguishing chromosomes.

\section{Anticipated Results:}

The production of a fluorescent probe that binds to the DNA sequence of interest and is able to be visualized by fluorescent microscopy. A minimum target size of $2.5 \mathrm{~kb}$ is recommended.

With the karyotyping cocktail, the final product should resemble Figure 3 once the image is merged and the chromosomes are identified and labeled.

\section{Time Considerations:}


7<smiles>C1CCCCC1</smiles>

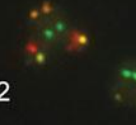

8

1

5

3

Figure 3 - Karyotype of Fast-Flowering Mini-Maize B. A karyotype produced using the methods described in this protocol. The white pseudocoloring of the far-red channel is produced by making a second RGB image with the same far-red channel capture representing the red, green, and blue channels. The RGB image of the real red, green, and blue channels is then merged with the image of the far-red channel in Photoshop. Each chromosome was then identified by its size and characteristic signals and labeled with its chromosome number. 


\section{DNA amplification:}

- Steps 1 to 3: Assembly of PCR reaction until completion of thermocycler protocol will take 3-4 $\mathrm{hr}$.

\section{DNA precipitation:}

- Steps 1 to 3: Assembly of ethanol precipitation reagents will take approximately $15 \mathrm{~min}$.

- Step 4: Incubation of ethanol precipitation will take from 30 min to overnight.

- Step 5: 30 min of centrifugation is required to pellet the DNA.

- Steps 6 to13: Wash steps and elution of the DNA pellet take about 20 $\min$.

\section{Checking DNA integrity:}

- Steps 1 to 2: The casting and assembly of the agarose gel takes about 30 $\min$.

- Step 3: Running of the agarose gel for 30 min will allow for good resolution of DNA.

- Step 4: 15 to $30 \mathrm{~min}$ are required to stain agarose gel in ethidium bromide.

- Steps 5 to 6: Visualization of the gel takes about 5 min. 
Nick translation:

Small targets:

- Steps 1 to 3: Assembly of nick translation reaction, as well as incubation, takes approximately $2.5 \mathrm{hr}$ to complete.

Large targets:

- Steps 1 to $3: 2.5 \mathrm{hr}$ is required to assemble and incubate the nick translation reaction.

\section{Probe purification:}

Column assembly:

- Steps 1 to 4: Assembly of column for probe purification takes 25 $\min$.

Column purification:

- Steps 1 to $2: 30$ to 45 min should be set aside for actually running the probe through the column.

\section{Ethanol precipitation:}

Small targets: 
- Steps 1 to 2: Assembly of reagents for ethanol precipitation takes $15 \min$.

- Step 3: Incubation of mixture takes from $2 \mathrm{hr}$ to overnight.

- Step 4: Centrifugation of ethanol precipitation mixture will take 30 $\min$.

- Steps 5 to 9: Washing of pelleted probe DNA will take approximately $15 \mathrm{~min}$.

Large targets:

- Steps 1 to 2: Assembly of reagents for ethanol precipitation takes $15 \min$.

- Step 3: Incubation of mixture takes from $2 \mathrm{hr}$ to overnight.

- Step 4: Centrifugation of ethanol precipitation mixture will take 30 $\min$.

- Steps 5 to 9: Washing of pelleted probe DNA will take approximately $15 \mathrm{~min}$.

\section{Karyotyping cocktail:}

Hybridization and slide finishing:

- Step 1: Adding probe to slides takes about 5-10 min depending on number of slides prepared. 
- Step 2: Adding prepared slides to boiling water takes approximately $5 \mathrm{~min}$.

- Step 3: Prepared slides are then incubated overnight.

- Steps 4 to 6 : Washing of probed slides in $2 \times S S C$ and drying takes approximately $50 \mathrm{~min}$.

- Step 7: Adding DAPI to slides takes approximately 5-10 min depending on number of prepared slides.

\section{Acknowledgements}

Research on this topic in the Birchler lab is supported by NSF grants IOS1339198 and IOS-1444514.

\section{References}

Albert P.S., Gao Z., Danilova T.V., and Birchler J.A. (2010) Diversity of chromosomal karyotypes in maize and its relatives. Cytogenet. Genome Res. 129:6-16.

Bishop R. (2010) Applications of fluorescence in situ hybridization (FISH) in detecting genetic aberrations of medical significance. Bioscience Horizons 3:8595.

Gaeta R.T., Danilova T.V., Zhao C., Masonbrink, R.E., McCaw,M.E., and Birchler, J.A. (2011) Recovery of a telomere-truncated chromosome via a compensating translocation in maize. Genome 54:184-195.

Kato A., Lamb J.C., and Birchler J.A. (2004) Chromosome painting using repetitive DNA sequences as probes for somatic chromosome identification in maize. Proc. Natl. Acad Sci. U.S.A. 101:13554-13559. 
Lamb J. and Birchler J. (2006) Retroelement genome painting: Cytological visualization of retroelement expansions in the genera Zea and Tripsacum. Genetics 173:1007-1021.

Lamb J., Meyer J., Corcoran B., Kato A., Han F., and Birchler J. (2007) Distinct chromosomal distributions of highly repetitive sequences in maize. Chromosome Res. 15:33-49.

Lamb J.C., Danilova T., Bauer M.J., Meyer J.M. Holland J.J., Jensen M.D., and Birchler J.A. (2007) Single-gene detection and karyotyping using small-target fluorescence in situ hybridization on maize somatic chromosomes. Genetics 175:1047-1058.

McCaw M., Swyers N., Graham N., Cody J., Zhao C., and Birchler J. (2016) Preparation of chromosomes from Zea mays. Curr. Protoc. Plant Biol. 1:501-508.

Yu W., Lamb J.C., Han F., and Birchler J.A. (2006) Telomere-mediated chromosomal truncation in maize. Proc. Natl. Acad Sci. U.S.A. 103:1733117336.

Yu W., Lamb J.C., Han F., and Birchler J.A. (2007) Cytological visualization of DNA transposons and their transposition pattern in somatic cells of maize. Genetics 175:31-39. 


\section{Appendix 5: Sequences of pNT1, pPZPGLP1, AC1E, and}

\section{AC2E}

\section{pNT1 Sequence}

AGTACTTTGATCCAACCCCTCCGCTGCTATAGTGCAGTCGGCTTCTGACGTT CAGTGCAGCCGTCTTCTGAAAACGACATGTCGCACAAGTCCTAAGTTACGC GACAGGCTGCCGCCCTGCCCTTTTCCTGGCGTTTTCTTGTCGCGTGTTTTA GTCGCATAAAGTAGAATACTTGCGACTAGAACCGGAGACATTACGCCATGA ACAAGAGCGCCGCCGCTGGCCTGCTGGGCTATGCCCGCGTCAGCACCGAC GACCAGGACTTGACCAACCAACGGGCCGAACTGCACGCGGCCGGCTGCAC CAAGCTGTTTTCCGAGAAGATCACCGGCACCAGGCGCGACCGCCCGGAGC TGGCCAGGATGCTTGACCACCTACGCCCTGGCGACGTTGTGACAGTGACC AGGCTAGACCGCCTGGCCCGCAGCACCCGCGACCTACTGGACATTGCCGA GCGCATCCAGGAGGCCGGCGCGGGCCTGCGTAGCCTGGCAGAGCCGTGG GCCGACACCACCACGCCGGCCGGCCGCATGGTGTTGACCGTGTTCGCCGG CATTGCCGAGTTCGAGCGTTCCCTAATCATCGACCGCACCCGGAGCGGGC GCGAGGCCGCCAAGGCCCGAGGCGTGAAGTTTGGCCCCCGCCCTACCCTC ACCCCGGCACAGATCGCGCACGCCCGCGAGCTGATCGACCAGGAAGGCC GCACCGTGAAAGAGGCGGCTGCACTGCTTGGCGTGCATCGCTCGACCCTG TACCGCGCACTTGAGCGCAGCGAGGAAGTGACGCCCACCGAGGCCAGGC GGCGCGGTGCCTTCCGTGAGGACGCATTGACCGAGGCCGACGCCCTGGC GGCCGCCGAGAATGAACGCCAAGAGGAACAAGCATGAAACCGCACCAGGA 
CGGCCAGGACGAACCGTTTTTCATTACCGAAGAGATCGAGGCGGAGATGAT CGCGGCCGGGTACGTGTTCGAGCCGCCCGCGCACGTCTCAACCGTGCGG CTGCATGAAATCCTGGCCGGTTTGTCTGATGCCAAGCTGGCGGCCTGGCC GGCCAGCTTGGCCGCTGAAGAAACCGAGCGCCGCCGTCTAAAAAGGTGAT GTGTATTTGAGTAAAACAGCTTGCGTCATGCGGTCGCTGCGTATATGATGC GATGAGTAAATAAACAAATACGCAAGGGGAACGCATGAAGGTTATCGCTGT ACTTAACCAGAAAGGCGGGTCAGGCAAGACGACCATCGCAACCCATCTAGC CCGCGCCCTGCAACTCGCCGGGGCCGATGTTCTGTTAGTCGATTCCGATCC CCAGGGCAGTGCCCGCGATTGGGCGGCCGTGCGGGAAGATCAACCGCTAA CCGTTGTCGGCATCGACCGCCCGACGATTGACCGCGACGTGAAGGCCATC GGCCGGCGCGACTTCGTAGTGATCGACGGAGCGCCCCAGGCGGCGGACT TGGCTGTGTCCGCGATCAAGGCAGCCGACTTCGTGCTGATTCCGGTGCAG CCAAGCCCTTACGACATATGGGCCACCGCCGACCTGGTGGAGCTGGTTAA GCAGCGCATTGAGGTCACGGATGGAAGGCTACAAGCGGCCTTTGTCGTGT CGCGGGCGATCAAAGGCACGCGCATCGGCGGTGAGGTTGCCGAGGCGCT GGCCGGGTACGAGCTGCCCATTCTTGAGTCCCGTATCACGCAGCGCGTGA GCTACCCAGGCACTGCCGCCGCCGGCACAACCGTTCTTGAATCAGAACCC GAGGGCGACGCTGCCCGCGAGGTCCAGGCGCTGGCCGCTGAAATTAAATC AAAACTCATTTGAGTTAATGAGGTAAAGAGAAAATGAGCAAAAGCACAAACA CGCTAAGTGCCGGCCGTCCGAGCGCACGCAGCAGCAAGGCTGCAACGTTG GCCAGCCTGGCAGACACGCCAGCCATGAAGCGGGTCAACTTTCAGTTGCC GGCGGAGGATCACACCAAGCTGAAGATGTACGCGGTACGCCAAGGCAAGA CCATTACCGAGCTGCTATCTGAATACATCGCGCAGCTACCAGAGTAAATGA 
GCAAATGAATAAATGAGTAGATGAATTTTAGCGGCTAAAGGAGGCGGCATG GAAAATCAAGAACAACCAGGCACCGACGCCGTGGAATGCCCCATGTGTGG AGGAACGGGCGGTTGGCCAGGCGTAAGCGGCTGGGTTGTCTGCCGGCCCT GCAATGGCACTGGAACCCCCAAGCCCGAGGAATCGGCGTGACGGTCGCAA ACCATCCGGCCCGGTACAAATCGGCGCGGCGCTGGGTGATGACCTGGTGG AGAAGTTGAAGGCCGCGCAGGCCGCCCAGCGGCAACGCATCGAGGCAGA AGCACGCCCCGGTGAATCGTGGCAAGCGGCCGCTGATCGAATCCGCAAAG AATCCCGGCAACCGCCGGCAGCCGGTGCGCCGTCGATTAGGAAGCCGCCC AAGGGCGACGAGCAACCAGATTTTTTCGTTCCGATGCTCTATGACGTGGGC ACCCGCGATAGTCGCAGCATCATGGACGTGGCCGTTTTCCGTCTGTCGAAG CGTGACCGACGAGCTGGCGAGGTGATCCGCTACGAGCTTCCAGACGGGCA CGTAGAGGTTTCCGCAGGGCCGGCCGGCATGGCCAGTGTGTGGGATTACG ACCTGGTACTGATGGCGGTTTCCCATCTAACCGAATCCATGAACCGATACC GGGAAGGGAAGGGAGACAAGCCCGGCCGCGTGTTCCGTCCACACGTTGC GGACGTACTCAAGTTCTGCCGGCGAGCCGATGGCGGAAAGCAGAAAGACG ACCTGGTAGAAACCTGCATTCGGTTAAACACCACGCACGTTGCCATGCAGC GTACGAAGAAGGCCAAGAACGGCCGCCTGGTGACGGTATCCGAGGGTGAA GCCTTGATTAGCCGCTACAAGATCGTAAAGAGCGAAACCGGGCGGCCGGA GTACATCGAGATCGAGCTAGCTGATTGGATGTACCGCGAGATCACAGAAGG CAAGAACCCGGACGTGCTGACGGTTCACCCCGATTACTTTTTGATCGATCC CGGCATCGGCCGTTTTCTCTACCGCCTGGCACGCCGCGCCGCAGGCAAGG CAGAAGCCAGATGGTTGTTCAAGACGATCTACGAACGCAGTGGCAGCGCC GGAGAGTTCAAGAAGTTCTGTTTCACCGTGCGCAAGCTGATCGGGTCAAAT 
GACCTGCCGGAGTACGATTTGAAGGAGGAGGCGGGGCAGGCTGGCCCGA TCCTAGTCATGCGCTACCGCAACCTGATCGAGGGCGAAGCATCCGCCGGTT CCTAATGTACGGAGCAGATGCTAGGGCAAATTGCCCTAGCAGGGGAAAAAG GTCGAAAAGGTCTCTTTCCTGTGGATAGCACGTACATTGGGAACCCAAAGC CGTACATTGGGAACCGGAACCCGTACATTGGGAACCCAAAGCCGTACATTG GGAACCGGTCACACATGTAAGTGACTGATATAAAAGAGAAAAAAGGCGATTT TTCCGCCTAAAACTCTTTAAAACTTATTAAAACTCTTAAAACCCGCCTGGCCT GTGCATAACTGTCTGGCCAGCGCACAGCCGAAGAGCTGCAAAAAGCGCCT ACCCTTCGGTCGCTGCGCTCCCTACGCCCCGCCGCTTCGCGTCGGCCTAT CGCGGCCGCTGGCCGCTCAAAAATGGCTGGCCTACGGCCAGGCAATCTAC CAGGGCGCGGACAAGCCGCGCCGTCGCCACTCGACCGCCGGCGCCCACA TCAAGGCACCCTGCCTCGCGCGTTTCGGTGATGACGGTGAAAACCTCTGAC ACATGCAGCTCCCGGAGACGGTCACAGCTTGTCTGTAAGCGGATGCCGGG AGCAGACAAGCCCGTCAGGGCGCGTCAGCGGGTGTTGGCGGGTGTCGGG GCGCAGCCATGACCCAGTCACGTAGCGATAGCGGAGTGTATACTGGCTTAA CTATGCGGCATCAGAGCAGATTGTACTGAGAGTGCACCATATGCGGTGTGA AATACCGCACAGATGCGTAAGGAGAAAATACCGCATCAGGCGCTCTTCCGC TTCCTCGCTCACTGACTCGCTGCGCTCGGTCGTTCGGCTGCGGCGAGCGG TATCAGCTCACTCAAAGGCGGTAATACGGTTATCCACAGAATCAGGGGATA ACGCAGGAAAGAACATGTGAGCAAAAGGCCAGCAAAAGGCCAGGAACCGT AAAAAGGCCGCGTTGCTGGCGTTTTTCCATAGGCTCCGCCCCCCTGACGAG CATCACAAAAATCGACGCTCAAGTCAGAGGTGGCGAAACCCGACAGGACTA TAAAGATACCAGGCGTTTCCCCCTGGAAGCTCCCTCGTGCGCTCTCCTGTT 
CCGACCCTGCCGCTTACCGGATACCTGTCCGCCTTTCTCCCTTCGGGAAGC GTGGCGCTTTCTCATAGCTCACGCTGTAGGTATCTCAGTTCGGTGTAGGTC GTTCGCTCCAAGCTGGGCTGTGTGCACGAACCCCCCGTTCAGCCCGACCG CTGCGCCTTATCCGGTAACTATCGTCTTGAGTCCAACCCGGTAAGACACGA CTTATCGCCACTGGCAGCAGCCACTGGTAACAGGATTAGCAGAGCGAGGTA TGTAGGCGGTGCTACAGAGTTCTTGAAGTGGTGGCCTAACTACGGCTACAC TAGAAGGACAGTATTTGGTATCTGCGCTCTGCTGAAGCCAGTTACCTTCGGA AAAAGAGTTGGTAGCTCTTGATCCGGCAAACAAACCACCGCTGGTAGCGGT GGTTTTTTTGTTTGCAAGCAGCAGATTACGCGCAGAAAAAAAGGATCTCAAG AAGATCCTTTGATCTTTTCTACGGGGTCTGACGCTCAGTGGAACGAAAACTC ACGTTAAGGGATTTTGGTCATGCATGATATATCTCCCAATTTGTGTAGGGCT TATTATGCACGCTTAAAAATAATAAAAGCAGACTTGACCTGATAGTTTGGCT GTGAGCAATTATGTGCTTAGTGCATCTAATCGCTTGAGTTAACGCCGGCGAA GCGGCGTCGGCTTGAACGAATTTCTAGCTAGACATTATTTGCCGACTACCTT GGTGATCTCGCCTTTCACGTAGTGGACAAATTCTTCCAACTGATCTGCGCGC GAGGCCAAGCGATCTTCTTCTTGTCCAAGATAAGCCTGTCTAGCTTCAAGTA TGACGGGCTGATACTGGGCCGGCAGGCGCTCCATTGCCCAGTCGGCAGCG ACATCCTTCGGCGCGATTTTGCCGGTTACTGCGCTGTACCAAATGCGGGAC AACGTAAGCACTACATTTCGCTCATCGCCAGCCCAGTCGGGCGGCGAGTTC CATAGCGTTAAGGTTTCATTTAGCGCCTCAAATAGATCCTGTTCAGGAACCG GATCAAAGAGTTCCTCCGCCGCTGGACCTACCAAGGCAACGCTATGTTCTC TTGCTTTTGTCAGCAAGATAGCCAGATCAATGTCGATCGTGGCTGGCTCGAA GATACCTGCAAGAATGTCATTGCGCTGCCATTCTCCAAATTGCAGTTCGCGC 
TTAGCTGGATAACGCCACGGAATGATGTCGTCGTGCACAACAATGGTGACT TCTACAGCGCGGAGAATCTCGCTCTCTCCAGGGGAAGCCGAAGTTTCCAAA AGGTCGTTGATCAAAGCTCGCCGCGTTGTTTCATCAAGCCTTACGGTCACC GTAACCAGCAAATCAATATCACTGTGTGGCTTCAGGCCGCCATCCACTGCG GAGCCGTACAAATGTACGGCCAGCAACGTCGGTTCGAGATGGCGCTCGAT GACGCCAACTACCTCTGATAGTTGAGTCGATACTTCGGCGATCACCGCTTC CCCCATGATGTTTAACTTTGTTTTAGGGCGACTGCCCTGCTGCGTAACATCG TTGCTGCTCCATAACATCAAACATCGACCCACGGCGTAACGCGCTTGCTGC TTGGATGCCCGAGGCATAGACTGTACCCCAAAAAAACATGTCATAACAAGAA GCCATGAAAACCGCCACTGCGCCGTTACCACCGCTGCGTTCGGTCAAGGTT CTGGACCAGTTGCGTGACGGCAGTTACGCTACTTGCATTACAGCTTACGAA CCGAACGAGGCTTATGTCCACTGGGTTCGTGCCCGAATTGATCACAGGCAG CAACGCTCTGTCATCGTTACAATCAACATGCTACCCTCCGCGAGATCATCCG TGTTTCAAACCCGGCAGCTTAGTTGCCGTTCTTCCGAATAGCATCGGTAACA TGAGCAAAGTCTGCCGCCTTACAACGGCTCTCCCGCTGACGCCGTCCCGG ACTGATGGGCTGCCTGTATCGAGTGGTGATTTTGTGCCGAGCTGCCGGTCG GGGAGCTGTTGGCTGGCTGGTGGCAGGATATATTGTGGTGTAAACAAATTG ACGCTTAGACAACTTAATAACACATTGCGGACGTTTTTAATGTACTGAATTAA CGCCGAATTGCTCTAGCCAATACGCAAACCGCCTCTCCCCGCGCGTTGGCC GATTCATTAATGCAGCTGGCACGACAGGTTTCCCGACTGGAAAGCGGGCAG TGAGCGCAACGCAATTAATGTGAGTTAGCTCACTCATTAGGCACCCCAGGC TTTACACTTTATGCTTCCGGCTCGTATGTTGTGTGGAATTGTGAGCGGATAA CAATTTCACACAGGAAACAGCTATGACCATGATTACGAATTGGCCCGGGAA 
GCTTGCATGCCTGCAGTGCAGCGTGACCCGGTCGTGCCCCTCTCTAGAGAT AATGAGCATTGCATGTCTAAGTTATAAAAAATTACCACATATTTTTTTTGTCAC ACTTGTTTGAAGTGCAGTTTATCTATCTTTATACATATATTTAAACTTTACTCT ACGAATAATATAATCTATAGTACTACAATAATATCAGTGTTTTAGAGAATCAT ATAAATGAACAGTTAGACATGGTCTAAAGGACAATTGAGTATTTTGACAACA GGACTCTACAGTTTTATCTTTTTAGTGTGCATGTGTTCTCCTTTTTTTTTTGCAA ATAGCTTCACCTATATAATACTTCATCCATTTTATTAGTACATCCATTTAGGGT TTAGGGTTAATGGTTTTTATAGACTAATTTTTTTAGTACATCTATTTTATTCTAT TTTAGCCTCTAAATTAAGAAAACTAAAACTCTATTTTAGTTTTTTTATTTAATAA TTTAGATATAAAATAGAATAAAATAAAGTGACTAAAAATTAAACAAATACCCTT TAAGAAATTAAAAAAACTAAGGAAACATTTTTCTTGTTTCGAGTAGATAATGC CAGCCTGTTAAACGCCGTCGACGAGTCTAACGGACACCAACCAGCGAACCA GCAGCGTCGCGTCGGGCCAAGCGAAGCAGACGGCACGGCATCTCTGTCGC TGCCTCTGGACCCCTCTCGAGAGTTCCGCTCCACCGTTGGACTTGCTCCGC TGTCGGCATCCAGAAATTGCGTGGCGGAGCGGCAGACGTGAGCCGGCACG GCAGGCGGCCTCCTCCTCCTCTCACGGCACGGCAGCTACGGGGGATTCCT TTCCCACCGCTCCTTCGCTTTCCCTTCCTCGCCCGCCGTAATAAATAGACAC CCCCTCCACACCСTCTTTCCCCAACCTCGTGTTGTTCGGAGCGCACACACA CACAACCAGATCTCCCCCAAATCCACCCGTCGGCACCTCCGCTTCAAGGTA CGCCGCTCGTCCTCCCCCCCCCCCCCTCTCTACCTTCTCTAGATCGGCGTT CCGGTCCATGGTTAGGGCCCGGTAGTTCTACTTCTGTTCATGTTTGTGTTAG ATCCGTGTTTGTGTTAGATCCGTGCTGCTAGCGTTCGTACACGGATGCGAC CTGTACGTCAGACACGTTCTGATTGCTAACTTGCCAGTGTTTCTCTTTGGGG 
AATCCTGGGATGGCTCTAGCCGTTCCGCAGACGGGATCGATTTCATGATTTT TTTTGTTTCGTTGCATAGGGTTTGGTTTGCCCTTTTCCTTTATTTCAATATATG CCGTGCACTTGTTTGTCGGGTCATCTTTTCATGCTTTTTTTTGTCTTGGTTGT GATGATGTGGTCTGGTTGGGCGGTCGTTCTAGATCGGAGTAGAATTCTGTT TCAAACTACCTGGTGGATTTATTAATTTTGGATCTGTATGTGTGTGCCATACA TATTCATAGTTACGAATTGAAGATGATGGATGGAAATATCGATCTAGGATAG GTATACATGTTGATGCGGGTTTTACTGATGCATATACAGAGATGCTTTTTGTT CGCTTGGTTGTGATGATGTGGTGTGGTTGGGCGGTCGTTCATTCGTTCTAG ATCGGAGTAGAATACTGTTTCAAACTACCTGGTGTATTTATTAATTTTTGGAAC TGTATGTGTGTGTCATACATCTTCATAGTTACGAGTTTAAGATGGATGGAAAT ATCGATCTAGGATAGGTATACATGTTGATGTGGGTTTTACTGATGCATATAC ATGATGGCATATGCAGCATCTATTCATATGCTCTAACCTTGAGTACCTATCTA TTATAATAAACAAGTATGTTTTATAATTATTTTGATCTTGATATACTTGGATGA TGGCATATGCAGCAGCTATATGTGGATTTTTTTAGCCCTGCCTTCATACGCT ATTTATTTGCTTGGTACTGTTTCTTTTGTCGATGCTCACCCTGTTGTTTGGTG TTACTTCTGCAGGTCGACTCTAGAGGATCCATAACTTCGTATAGCATACATT ATACGAAGTTATACTAGTGGATCCATCGATTAGGAAGTAACCATGAGCCCAG AACGACGCCCGGCCGACATCCGCCGTGCCACCGAGGCGGACATGCCGGC GGTCTGCACCATCGTCAACCACTACATCGAGACAAGCACGGTCAACTTCCG TACCGAGCCGCAGGAACCGCAGGAGTGGACGGACGACCTCGTCCGTCTGC GGGAGCGCTATCCCTGGCTCGTCGCCGAGGTGGACGGCGAGGTCGCCGG CATCGCCTACGCGGGCCCCTGGAAGGCACGCAACGCCTACGACTGGACGG CCGAGTCGACCGTGTACGTCTCCCCCCGCCACCAGCGGACGGGACTGGGC 
TCCACGCTCTACACCCACCTGCTGAAGTCCCTGGAGGCACAGGGCTTCAAG AGCGTGGTCGCTGTCATCGGGCTGCCCAACGACCCGAGCGTGCGCATGCA CGAGGCGCTCGGATATGCCCCCCGCGGCATGCTGCGGGCGGCCGGCTTC AAGCACGGGAACTGGCATGACGTGGGTTTCTGGCAGCTGGACTTCAGCCT GCCGGTACCGCCCCGTCCGGTCCTGCCCGTCACCGAGATCTGATCCGTCG ACCTGCAGATCGTTCAAACATTTGGCAATAAAGTTTCTTAAGATTGAATCCTG TTGCCGGTCTTGCGATGATTATCATATAATTTCTGTTGAATTACGTTAAGCAT GTAATAATTAACATGTAATGCATGACGTTATTTATGAGATGGGTTTTTATGAT TAGAGTCCCGCAATTATACATTTAATACGCGATAGAAAACAAAATATAGCGC GCAAACTAGGATAAATTATCGCGCGCGGTGTCATCTATGTTACTAGATCCGA TGATAAGCTGTCAAACATGAGAATTCGATTTTGCATGATATCTTTCCCATAAC TTCGTATAGCATACATTATACGAAGTTATGGTTTTCCCCCAACTGGGGTAAC CTTTGAGTTCTCTCAGTTGGGGGGGCGGCCGCAAAGGCGCGCCCCTCCGC GCCGAGGATTAACCCTCACTAAAGGGAACAAAAGCTGGGTACCGGGCCCC CCCTCGAGGTCGACGGTATCGATAAGCTTGCATGCCTGCAGGTGATGAGAG ATCGTTCAAACATTTGGCAATAAAGTTTCTTAAGATTGAATCCTGTTGCCGGT CTTGCGATGATTATCATATAATTTCTGTTGAATTACGTTAAGCATGTAATAATT AACATGTAATGCATGACGTTATTTATGAGATGGGTTTTTATGATTAGAGTCCC GCAATTATACATTTAATACGCGATAGAAAACAAAATATAGCGCGCAAACTAG GATAAATTATCGCGCGCGGTGTCATCTATGTTACTAGATCTTGACGAGACCT GCAGGTCCGATTGAGACTTTTCAACAAAGGGTAATATCCGGAAACCTCCTC GGATTCCATTGCCCAGCTATCTGTCACTTTATTGTGAAGATAGTGGAAAAGG AAGGTGGCTCCTACAAATGCCATCATTGCGATAAAGGAAAGGCCATCGTTG 
AAGATGCCTCTGCCGACAGTGGTCCCAAAGATGGACCCCCACCCACGAGG AGCATCGTGGAAAAAGAAGACGTTCCAACCACGTCTTCAAAGCAAGTGGAT TGATGTGATGGTCCGATTGAGACTTTTCAACAAAGGGTAATATCCGGAAACC TCCTCGGATTCCATTGCCCAGCTATCTGTCACTTTATTGTGAAGATAGTGGA AAAGGAAGGTGGCTCCTACAAATGCCATCATTGCGATAAAGGAAAGGCCAT CGTTGAAGATGCCTCTGCCGACAGTGGTCCCAAAGATGGACCCCCACCCAC GAGGAGCATCGTGGAAAAAGAAGACGTTCCAACCACGTCTTCAAAGCAAGT GGATTGATGTGATATCTCCACTGACGTAAGGGATGACGCACAATCCCACTAT CCTTCGCAAGACCCTTCCTCTATATAAGGAAGTTCATTTCATTTGGAGAGGA GATCTTTTTATTTTTAATTTTCTTTCAAATACTTCCACCATGGTGAGCAAGGG CGAGGAGCTGTTCACCGGGGTGGTGCCCATCCTGGTCGAGCTGGACGGCG ACGTAAACGGCCACAAGTTCAGCGTGTCCGGCGAGGGCGAGGGCGATGCC ACCTACGGCAAGCTGACCCTGAAGTTCATCTGCACCACCGGCAAGCTGCCC GTGCCCTGGCCCACCCTCGTGACCACCCTGACCTACGGCGTGCAGTGCTT CAGCCGCTACCCCGACCACATGAAGCAGCACGACTTCTTCAAGTCCGCCAT GCCCGAAGGCTACGTCCAGGAGCGCACCATCTTCTTCAAGGACGACGGCA ACTACAAGACCCGCGCCGAGGTGAAGTTCGAGGGCGACACCCTGGTGAAC CGCATCGAGCTGAAGGGCATCGACTTCAAGGAGGACGGCAACATCCTGGG GCACAAGCTGGAGTACAACTACAACAGCCACAACGTCTATATCATGGCCGA CAAGCAGAAGAACGGCATCAAGGTGAACTTCAAGATCCGCCACAACATCGA GGACGGCAGCGTGCAGCTCGCCGACCACTACCAGCAGAACACCCCCATCG GCGACGGCCCCGTGCTGCTGCCCGACAACCACTACCTGAGCACCCAGTCC GCCCTGAGCAAAGACCCCAAGGAGAAGCGCGATCACATGGTCCTGCTGGA 
GTTCGTGACCGCCGCCGGGATCACTCTCGGCATGGACGAGCCGTACAAGT AAGAGCTCGGATCCCCGATCGTTCAAACATTTGGCAATAAAGTTTCTTAAGA TTGAATCCTGTTGCCGGTCTTGCGATGATTATCATATAATTTCTGTTGAATTA CGTTAAGCATGTAATAATTAACATGTAATGCATGACGTTATTTATGAGATGGG TTTTTATGATTAGAGTCCCGCAATTATACATTTAATACGCGATAGAAAACAAA ATATAGCGCGCAAACTAGGATAAATTATCGCGCGCGGTGTCATCTATGTTAC TAGATCGGGAATTCCTGCAGCCCGGGGGATCCACTAGTTCTAGAGCGGCC GCCACCGCGGTGGAGCTCCAATTCGCCCTATAGTGAGTCGTATTATCCCGG CGCGCCAAAAAAAGGCCGGCCGACGTCTTAATTAAGGGCCCAATTGGCCAC GTACGTCTAGATCTGGCGCGCCTCGAGGATCCATGGCCGCGGGATTCCCT CGGGTCCCACAGCGCTTTATTTAGGGTTTAGGGTTTAGGGTTTAGGGTTTAG GGTTTAGGGTTTAGGGTTTAGGGTTTAGGGTTTAGGGTTTAGGGTTTAGGGT TTAGGGTTTAGGGTTTAGGGTTTAGGGTTTAGGGTTTAGGGTTTAGGGTTTA GGGTTTAGGGTTTAGGGTTTAGGGTTTAGGGTTTAGGGTTTAGGGTTTAGG GTTTAGGGTTTAGGGTTTAGGGTTTAGGGTTTAGGGTTTAGGGTTTAGGGTT TAGGGTTTAGGGTTTAGGGTTTAGGGTTTAGGGTTTAGGGTTTAGGGTTTAG GGTTTAGGGTTTAGGGTTTAGGGTTTAGGGTTTAGGGTTTAGGGTTTAGGGT TTAGGGTTTAGGGTTTAGGGTTTAGGGTTTAGGGTTTAGGGTTTAGGGTTTA GGGTTTAGGGTTTAGGGTTTAGGGTTTAGGGTTTAGGGTTTAGGGTTTAGG GTTTAGGGTTTAGGGTTTAGGGTTTAGGGTTTAGGGTTTAGGGTTTAGGGTT TAGGGTTTAGGGTTTAGGGTTTAGGGTTTAGGGTTTAGGGTTTAGGGTTTAG GGTTTAGGGTTTAGGGTTTAGGGTTTAGGGTTTAGGGTTTAGGGTTTAGGGT TTAGGGTTTAGGGTTTAGGGTTTAGGGTTTAGGGTTTAGGGTTTAGGGTTTA 
GGGTTTAGGGTTTAGGGTTTAGGGTTTAGGGTTTAGGGTTTAGGGTTTAGG GTTTAGGGTTTAGGGTTTAGGGTTTAGGGTTTAGGGTTTAGGGTTTAGGGTT TAGGGTTTAGGGTTTAGGGTTTAGGGTTTAGGGTTTAGGGTTTAGGGTTTAG GGTTTAGGGTTTAGGGTTTAGGGTTTAGGGTTTAGGGTTTAGGGTTTAGGGT TTAGGGTTTAGGGTTTAGGGTTTAGGGTTTAGGGTTTAGGGTTTAGGGTTTA GGGTTTAGGGTTTAGGGTTTAGGGTTTAGGGTTTAGGGTTTAGGGTTTAGG GTTTAGGGTTTAGGGTTTAGGGTTTAGGGTTTAGGGTTTAGGGTTTAGGGTT TAGGGTTTAGGGTTTAGGGTTTAGGGTTTAGGGTTTAGGGTTTAGGGTTTAG GGTTTAGGGTTTAGGGTTTAGGGTTTAGGGTTTAGGGTTTAGGGTTTAGGGT TTAGGGTTTAGGGTTTAGGGTTTAGGGTTTAGGGTTTAGGGTTTAGGGTTTA GGGTTTAGGGTTTAGGGTTTAGGGTTTAGGGTTTAGGGTTTAGGGTTTAGG GTTTAGGGTTTAGGGTTTAGGGTTTAGGGTTTAGGGTTTAGGGTTTAGGGTT TAGGGTTTAGGGTTTAGGGTTTAGGGTTTAGGGTTTAGGGTTTAGGGTTTAG GGTTTAGGGTTTAGGGTTTAGGGTTTAGGGTTTAGGGTTTAGGGTTTAGGGT TTAGGGTTTAGGGTTTAGGGTTTAGGGTTTAGGGTTTAGGGTTTAGGGTTTA GGGTTTAGGGTTTAGGGTTTAGGGTTTAGGGTTTAGGGTTTAGGGTTTAGG GTTTAGGGTTTAGGGTTTAGGGTTTAGGGTTTAGGGTTTAGGGTTTAGGGTT TAGGGTTTAGGGTTTAGGGTTTAGGGTTTAGGGTTTAGGGTTTAGGGTTTAG GGTTTAGGGTTTAGGGTTTAGGGTTTAGGGTTTAGGGTTTAGGGTTTAGGGT TTAGGGTTTAGGGTTTAGGGTTTAGGGTTTAGGGTTTAGGGTTTAGGGTTTA GGGTTTAGGGTTTAGGGTTTAGGGTTTAGGGTTTAGGGTTTAGGGTTTAGG GTTTAGGGTTTAGGGTTTAGGGTTTAGGGTTTAGGGTTTAGGGTTTAGGGTT TAGGGTTTAGGGTTTAGGGTTTAGGGTTTAGGGTTTAGGGTTTAGGGTTTAG 
GGTTTAGGGTTTAGGGTTTAGGGTTTAGGGTTTAGGGTTTAGGGTTTAGGGT TTAGGGTTTAGGGTTTAGGGTTTAGGGTTTAGGGTTTAGGGTTTAGGGTTTA GGGTTTAGGGTTTAGGGTTTAGGGTTTAGGGTTTAGGGTTTAGGGTTTAGG GTTTAGGGTTTAGGGTTTAGGGTTTAGGGTTTAGGGTTTAGGGTTTAGGGTT TAGGGTTTAGGGTTTAGGGTTTAGGGTTTAGGGTTTAGGGTTTAGGGTTTAG GGTTTAGGGTTTAGGGTTTAGGGTTTAGGGTTTAGGGTTTAGGGTTTAGGGT TTAGGGTTTAGGGTTTAGGGTTTAGGGTTTAGGGTTTAGGGTTTAGGGTTTA GGGTTTAGGGTTTAGGGTTTAGGGTTTAGGGTTTAGGGTTTAGGGTTTAGG GTTTAGGGTTTAGGGTTTAGGGTTTAGGGTTTAGGGTTTAGGGTTTAGGGTT TAGGGTTTAGGGTTTAGGGTTTAGGGTTTAGGGTTTAGGGTTTAGGGTTTAG GGTTTAGGGTTTAGGGTTTAGGGTTTAGGGTTTAGGGTTTAGGGTTTAGGGT TTAGGGTTTAGGGTTTAGGGTTTAGGGTTTAGGGTTTAGGGTTTAGGGTTTA GGGTTTAGGGTTTAGGGTTTAGGGTTTAGGGTTTAGGGTTTAGGGTTTAGG GTTTAGGGTTTAGGGTTTAGGGTTTAGGGTTTAGGGTTTAGGGTTTAGGGTT TAGGGTTTAGGGTTTAGGGTTTAGGGTGACCTGCAGCCCAAGCTCTCGGGA AATCACTAGTGATCGATAAACTATCAGTGTTTGACAGGATATATTGGCGGGT AAACCTAAGAGAAAAGAGCGTTTATTAGAATAACGGATATTTAAAAGGGCGT GAAAAGGTTTATCCGTTCGTCCATTTGTATGTGCATGCCAACCACAGGGTTC CCCTCGGGATCAA

pPZPGLP1

AGTACTTTGATCCAACCCCTCCGCTGCTATAGTGCAGTCGGCTTCTGACGTT CAGTGCAGCCGTCTTCTGAAAACGACATGTCGCACAAGTCCTAAGTTACGC GACAGGCTGCCGCCCTGCCCTTTTCCTGGCGTTTTCTTGTCGCGTGTTTTA 
GTCGCATAAAGTAGAATACTTGCGACTAGAACCGGAGACATTACGCCATGA ACAAGAGCGCCGCCGCTGGCCTGCTGGGCTATGCCCGCGTCAGCACCGAC GACCAGGACTTGACCAACCAACGGGCCGAACTGCACGCGGCCGGCTGCAC CAAGCTGTTTTCCGAGAAGATCACCGGCACCAGGCGCGACCGCCCGGAGC TGGCCAGGATGCTTGACCACCTACGCCCTGGCGACGTTGTGACAGTGACC AGGCTAGACCGCCTGGCCCGCAGCACCCGCGACCTACTGGACATTGCCGA GCGCATCCAGGAGGCCGGCGCGGGCCTGCGTAGCCTGGCAGAGCCGTGG GCCGACACCACCACGCCGGCCGGCCGCATGGTGTTGACCGTGTTCGCCGG CATTGCCGAGTTCGAGCGTTCCCTAATCATCGACCGCACCCGGAGCGGGC GCGAGGCCGCCAAGGCCCGAGGCGTGAAGTTTGGCCCCCGCCCTACCCTC ACCCCGGCACAGATCGCGCACGCCCGCGAGCTGATCGACCAGGAAGGCC GCACCGTGAAAGAGGCGGCTGCACTGCTTGGCGTGCATCGCTCGACCCTG TACCGCGCACTTGAGCGCAGCGAGGAAGTGACGCCCACCGAGGCCAGGC GGCGCGGTGCCTTCCGTGAGGACGCATTGACCGAGGCCGACGCCCTGGC GGCCGCCGAGAATGAACGCCAAGAGGAACAAGCATGAAACCGCACCAGGA CGGCCAGGACGAACCGTTTTTCATTACCGAAGAGATCGAGGCGGAGATGAT CGCGGCCGGGTACGTGTTCGAGCCGCCCGCGCACGTCTCAACCGTGCGG CTGCATGAAATCCTGGCCGGTTTGTCTGATGCCAAGCTGGCGGCCTGGCC GGCCAGCTTGGCCGCTGAAGAAACCGAGCGCCGCCGTCTAAAAAGGTGAT GTGTATTTGAGTAAAACAGCTTGCGTCATGCGGTCGCTGCGTATATGATGC GATGAGTAAATAAACAAATACGCAAGGGGAACGCATGAAGGTTATCGCTGT ACTTAACCAGAAAGGCGGGTCAGGCAAGACGACCATCGCAACCCATCTAGC CCGCGCCCTGCAACTCGCCGGGGCCGATGTTCTGTTAGTCGATTCCGATCC 
CCAGGGCAGTGCCCGCGATTGGGCGGCCGTGCGGGAAGATCAACCGCTAA CCGTTGTCGGCATCGACCGCCCGACGATTGACCGCGACGTGAAGGCCATC GGCCGGCGCGACTTCGTAGTGATCGACGGAGCGCCCCAGGCGGCGGACT TGGCTGTGTCCGCGATCAAGGCAGCCGACTTCGTGCTGATTCCGGTGCAG CCAAGCCCTTACGACATATGGGCCACCGCCGACCTGGTGGAGCTGGTTAA GCAGCGCATTGAGGTCACGGATGGAAGGCTACAAGCGGCCTTTGTCGTGT CGCGGGCGATCAAAGGCACGCGCATCGGCGGTGAGGTTGCCGAGGCGCT GGCCGGGTACGAGCTGCCCATTCTTGAGTCCCGTATCACGCAGCGCGTGA GCTACCCAGGCACTGCCGCCGCCGGCACAACCGTTCTTGAATCAGAACCC GAGGGCGACGCTGCCCGCGAGGTCCAGGCGCTGGCCGCTGAAATTAAATC AAAACTCATTTGAGTTAATGAGGTAAAGAGAAAATGAGCAAAAGCACAAACA CGCTAAGTGCCGGCCGTCCGAGCGCACGCAGCAGCAAGGCTGCAACGTTG GCCAGCCTGGCAGACACGCCAGCCATGAAGCGGGTCAACTTTCAGTTGCC GGCGGAGGATCACACCAAGCTGAAGATGTACGCGGTACGCCAAGGCAAGA CCATTACCGAGCTGCTATCTGAATACATCGCGCAGCTACCAGAGTAAATGA GCAAATGAATAAATGAGTAGATGAATTTTAGCGGCTAAAGGAGGCGGCATG GAAAATCAAGAACAACCAGGCACCGACGCCGTGGAATGCCCCATGTGTGG AGGAACGGGCGGTTGGCCAGGCGTAAGCGGCTGGGTTGTCTGCCGGCCCT GCAATGGCACTGGAACCCCCAAGCCCGAGGAATCGGCGTGACGGTCGCAA ACCATCCGGCCCGGTACAAATCGGCGCGGCGCTGGGTGATGACCTGGTGG AGAAGTTGAAGGCCGCGCAGGCCGCCCAGCGGCAACGCATCGAGGCAGA AGCACGCCCCGGTGAATCGTGGCAAGCGGCCGCTGATCGAATCCGCAAAG AATCCCGGCAACCGCCGGCAGCCGGTGCGCCGTCGATTAGGAAGCCGCCC 
AAGGGCGACGAGCAACCAGATTTTTTCGTTCCGATGCTCTATGACGTGGGC ACCCGCGATAGTCGCAGCATCATGGACGTGGCCGTTTTCCGTCTGTCGAAG CGTGACCGACGAGCTGGCGAGGTGATCCGCTACGAGCTTCCAGACGGGCA CGTAGAGGTTTCCGCAGGGCCGGCCGGCATGGCCAGTGTGTGGGATTACG ACCTGGTACTGATGGCGGTTTCCCATCTAACCGAATCCATGAACCGATACC GGGAAGGGAAGGGAGACAAGCCCGGCCGCGTGTTCCGTCCACACGTTGC GGACGTACTCAAGTTCTGCCGGCGAGCCGATGGCGGAAAGCAGAAAGACG ACCTGGTAGAAACCTGCATTCGGTTAAACACCACGCACGTTGCCATGCAGC GTACGAAGAAGGCCAAGAACGGCCGCCTGGTGACGGTATCCGAGGGTGAA GCCTTGATTAGCCGCTACAAGATCGTAAAGAGCGAAACCGGGCGGCCGGA GTACATCGAGATCGAGCTAGCTGATTGGATGTACCGCGAGATCACAGAAGG CAAGAACCCGGACGTGCTGACGGTTCACCCCGATTACTTTTTGATCGATCC CGGCATCGGCCGTTTTCTCTACCGCCTGGCACGCCGCGCCGCAGGCAAGG CAGAAGCCAGATGGTTGTTCAAGACGATCTACGAACGCAGTGGCAGCGCC GGAGAGTTCAAGAAGTTCTGTTTCACCGTGCGCAAGCTGATCGGGTCAAAT GACCTGCCGGAGTACGATTTGAAGGAGGAGGCGGGGCAGGCTGGCCCGA TCCTAGTCATGCGCTACCGCAACCTGATCGAGGGCGAAGCATCCGCCGGTT CCTAATGTACGGAGCAGATGCTAGGGCAAATTGCCCTAGCAGGGGAAAAAG GTCGAAAAGGTCTCTTTCCTGTGGATAGCACGTACATTGGGAACCCAAAGC CGTACATTGGGAACCGGAACCCGTACATTGGGAACCCAAAGCCGTACATTG GGAACCGGTCACACATGTAAGTGACTGATATAAAAGAGAAAAAAGGCGATTT TTCCGCCTAAAACTCTTTAAAACTTATTAAAACTCTTAAAACCCGCCTGGCCT GTGCATAACTGTCTGGCCAGCGCACAGCCGAAGAGCTGCAAAAAGCGCCT 
ACCCTTCGGTCGCTGCGCTCCCTACGCCCCGCCGCTTCGCGTCGGCCTAT CGCGGCCGCTGGCCGCTCAAAAATGGCTGGCCTACGGCCAGGCAATCTAC CAGGGCGCGGACAAGCCGCGCCGTCGCCACTCGACCGCCGGCGCCCACA TCAAGGCACCCTGCCTCGCGCGTTTCGGTGATGACGGTGAAAACCTCTGAC ACATGCAGCTCCCGGAGACGGTCACAGCTTGTCTGTAAGCGGATGCCGGG AGCAGACAAGCCCGTCAGGGCGCGTCAGCGGGTGTTGGCGGGTGTCGGG GCGCAGCCATGACCCAGTCACGTAGCGATAGCGGAGTGTATACTGGCTTAA CTATGCGGCATCAGAGCAGATTGTACTGAGAGTGCACCATATGCGGTGTGA AATACCGCACAGATGCGTAAGGAGAAAATACCGCATCAGGCGCTCTTCCGC TTCCTCGCTCACTGACTCGCTGCGCTCGGTCGTTCGGCTGCGGCGAGCGG TATCAGCTCACTCAAAGGCGGTAATACGGTTATCCACAGAATCAGGGGATA ACGCAGGAAAGAACATGTGAGCAAAAGGCCAGCAAAAGGCCAGGAACCGT AAAAAGGCCGCGTTGCTGGCGTTTTTCCATAGGCTCCGCCCCCCTGACGAG CATCACAAAAATCGACGCTCAAGTCAGAGGTGGCGAAACCCGACAGGACTA TAAAGATACCAGGCGTTTCCCCCTGGAAGCTCCCTCGTGCGCTCTCCTGTT CCGACCCTGCCGCTTACCGGATACCTGTCCGCCTTTCTCCCTTCGGGAAGC GTGGCGCTTTCTCATAGCTCACGCTGTAGGTATCTCAGTTCGGTGTAGGTC GTTCGCTCCAAGCTGGGCTGTGTGCACGAACCCCCCGTTCAGCCCGACCG CTGCGCCTTATCCGGTAACTATCGTCTTGAGTCCAACCCGGTAAGACACGA CTTATCGCCACTGGCAGCAGCCACTGGTAACAGGATTAGCAGAGCGAGGTA TGTAGGCGGTGCTACAGAGTTCTTGAAGTGGTGGCCTAACTACGGCTACAC TAGAAGGACAGTATTTGGTATCTGCGCTCTGCTGAAGCCAGTTACCTTCGGA AAAAGAGTTGGTAGCTCTTGATCCGGCAAACAAACCACCGCTGGTAGCGGT 
GGTTTTTTTGTTTGCAAGCAGCAGATTACGCGCAGAAAAAAAGGATCTCAAG AAGATCCTTTGATCTTTTCTACGGGGTCTGACGCTCAGTGGAACGAAAACTC ACGTTAAGGGATTTTGGTCATGCATGATATATCTCCCAATTTGTGTAGGGCT TATTATGCACGCTTAAAAATAATAAAAGCAGACTTGACCTGATAGTTTGGCT GTGAGCAATTATGTGCTTAGTGCATCTAATCGCTTGAGTTAACGCCGGCGAA GCGGCGTCGGCTTGAACGAATTTCTAGCTAGACATTATTTGCCGACTACCTT GGTGATCTCGCCTTTCACGTAGTGGACAAATTCTTCCAACTGATCTGCGCGC GAGGCCAAGCGATCTTCTTCTTGTCCAAGATAAGCCTGTCTAGCTTCAAGTA TGACGGGCTGATACTGGGCCGGCAGGCGCTCCATTGCCCAGTCGGCAGCG ACATCCTTCGGCGCGATTTTGCCGGTTACTGCGCTGTACCAAATGCGGGAC AACGTAAGCACTACATTTCGCTCATCGCCAGCCCAGTCGGGCGGCGAGTTC CATAGCGTTAAGGTTTCATTTAGCGCCTCAAATAGATCCTGTTCAGGAACCG GATCAAAGAGTTCCTCCGCCGCTGGACCTACCAAGGCAACGCTATGTTCTC TTGCTTTTGTCAGCAAGATAGCCAGATCAATGTCGATCGTGGCTGGCTCGAA GATACCTGCAAGAATGTCATTGCGCTGCCATTCTCCAAATTGCAGTTCGCGC TTAGCTGGATAACGCCACGGAATGATGTCGTCGTGCACAACAATGGTGACT TCTACAGCGCGGAGAATCTCGCTCTCTCCAGGGGAAGCCGAAGTTTCCAAA AGGTCGTTGATCAAAGCTCGCCGCGTTGTTTCATCAAGCCTTACGGTCACC GTAACCAGCAAATCAATATCACTGTGTGGCTTCAGGCCGCCATCCACTGCG GAGCCGTACAAATGTACGGCCAGCAACGTCGGTTCGAGATGGCGCTCGAT GACGCCAACTACCTCTGATAGTTGAGTCGATACTTCGGCGATCACCGCTTC CCCCATGATGTTTAACTTTGTTTTAGGGCGACTGCCCTGCTGCGTAACATCG TTGCTGCTCCATAACATCAAACATCGACCCACGGCGTAACGCGCTTGCTGC 
TTGGATGCCCGAGGCATAGACTGTACCCCAAAAAAACATGTCATAACAAGAA GCCATGAAAACCGCCACTGCGCCGTTACCACCGCTGCGTTCGGTCAAGGTT CTGGACCAGTTGCGTGACGGCAGTTACGCTACTTGCATTACAGCTTACGAA CCGAACGAGGCTTATGTCCACTGGGTTCGTGCCCGAATTGATCACAGGCAG CAACGCTCTGTCATCGTTACAATCAACATGCTACCCTCCGCGAGATCATCCG TGTTTCAAACCCGGCAGCTTAGTTGCCGTTCTTCCGAATAGCATCGGTAACA TGAGCAAAGTCTGCCGCCTTACAACGGCTCTCCCGCTGACGCCGTCCCGG ACTGATGGGCTGCCTGTATCGAGTGGTGATTTTGTGCCGAGCTGCCGGTCG GGGAGCTGTTGGCTGGCTGGTGGCAGGATATATTGTGGTGTAAACAAATTG ACGCTTAGACAACTTAATAACACATTGCGGACGTTTTTAATGTACTGAATTAA CGCCGAATTGCTCTAGCCAATACGCAAACCGCCTCTCCCCGCGCGTTGGCC GATTCATTAATGCAGCTGGCACGACAGGTTTCCCGACTGGAAAGCGGGCAG TGAGCGCAACGCAATTAATGTGAGTTAGCTCACTCATTAGGCACCCCAGGC TTTACACTTTATGCTTCCGGCTCGTATGTTGTGTGGAATTGTGAGCGGATAA CAATTTCACACAGGAAACAGCTATGACCATGATTACGAATTGGCCCGGGAA GCTTGCATGCCTGCAGTGCAGCGTGACCCGGTCGTGCCCCTCTCTAGAGAT AATGAGCATTGCATGTCTAAGTTATAAAAAATTACCACATATTTTTTTTGTCAC ACTTGTTTGAAGTGCAGTTTATCTATCTTTATACATATATTTAAACTTTACTCT ACGAATAATATAATCTATAGTACTACAATAATATCAGTGTTTTAGAGAATCAT ATAAATGAACAGTTAGACATGGTCTAAAGGACAATTGAGTATTTTGACAACA GGACTCTACAGTTTTATCTTTTTAGTGTGCATGTGTTCTCCTTTTTTTTTGCAA ATAGCTTCACCTATATAATACTTCATCCATTTTATTAGTACATCCATTTAGGGT TTAGGGTTAATGGTTTTTATAGACTAATTTTTTTAGTACATCTATTTTATTCTAT 
TTTAGCCTCTAAATTAAGAAAACTAAAACTCTATTTTAGTTTTTTTATTTAATAA TTTAGATATAAAATAGAATAAAATAAAGTGACTAAAAATTAAACAAATACCCTT TAAGAAATTAAAAAAACTAAGGAAACATTTTTCTTGTTTCGAGTAGATAATGC CAGCCTGTTAAACGCCGTCGACGAGTCTAACGGACACCAACCAGCGAACCA GCAGCGTCGCGTCGGGCCAAGCGAAGCAGACGGCACGGCATCTCTGTCGC TGCCTCTGGACCCCTCTCGAGAGTTCCGCTCCACCGTTGGACTTGCTCCGC TGTCGGCATCCAGAAATTGCGTGGCGGAGCGGCAGACGTGAGCCGGCACG GCAGGCGGCCTCCTCCTCCTCTCACGGCACGGCAGCTACGGGGGATTCCT TTCCCACCGCTCCTTCGCTTTCCCTTCCTCGCCCGCCGTAATAAATAGACAC CCСCTCCACACCСTCTTTCCCCAACCTCGTGTTGTTCGGAGCGCACACACA CACAACCAGATCTCCCCCAAATCCACCCGTCGGCACCTCCGCTTCAAGGTA CGCCGCTCGTCCTCCCCCCCCCCCCCTCTCTACCTTCTCTAGATCGGCGTT CCGGTCCATGGTTAGGGCCCGGTAGTTCTACTTCTGTTCATGTTTGTGTTAG ATCCGTGTTTGTGTTAGATCCGTGCTGCTAGCGTTCGTACACGGATGCGAC CTGTACGTCAGACACGTTCTGATTGCTAACTTGCCAGTGTTTCTCTTTGGGG AATCCTGGGATGGCTCTAGCCGTTCCGCAGACGGGATCGATTTCATGATTTT TTTTGTTTCGTTGCATAGGGTTTGGTTTGCCCTTTTCCTTTATTTCAATATATG CCGTGCACTTGTTTGTCGGGTCATCTTTTCATGCTTTTTTTTGTCTTGGTTGT GATGATGTGGTCTGGTTGGGCGGTCGTTCTAGATCGGAGTAGAATTCTGTT TCAAACTACCTGGTGGATTTATTAATTTTGGATCTGTATGTGTGTGCCATACA TATTCATAGTTACGAATTGAAGATGATGGATGGAAATATCGATCTAGGATAG GTATACATGTTGATGCGGGTTTTACTGATGCATATACAGAGATGCTTTTTGTT CGCTTGGTTGTGATGATGTGGTGTGGTTGGGCGGTCGTTCATTCGTTCTAG 
ATCGGAGTAGAATACTGTTTCAAACTACCTGGTGTATTTATTAATTTTGGAAC TGTATGTGTGTGTCATACATCTTCATAGTTACGAGTTTAAGATGGATGGAAAT ATCGATCTAGGATAGGTATACATGTTGATGTGGGTTTTACTGATGCATATAC ATGATGGCATATGCAGCATCTATTCATATGCTCTAACCTTGAGTACCTATCTA TTATAATAAACAAGTATGTTTTATAATTATTTTGATCTTGATATACTTGGATGA TGGCATATGCAGCAGCTATATGTGGATTTTTTTAGCCCTGCCTTCATACGCT ATTTATTTGCTTGGTACTGTTTCTTTTGTCGATGCTCACCCTGTTGTTTGGTG TTACTTCTGCAGGTCGACTCTAGAGGATCCATAACTTCGTATAGCATACATT ATACGAAGTTATACTAGTGGATCCATCGATTAGGAAGTAACCATGAGCCCAG AACGACGCCCGGCCGACATCCGCCGTGCCACCGAGGCGGACATGCCGGC GGTCTGCACCATCGTCAACCACTACATCGAGACAAGCACGGTCAACTTCCG TACCGAGCCGCAGGAACCGCAGGAGTGGACGGACGACCTCGTCCGTCTGC GGGAGCGCTATCCCTGGCTCGTCGCCGAGGTGGACGGCGAGGTCGCCGG CATCGCCTACGCGGGCCCCTGGAAGGCACGCAACGCCTACGACTGGACGG CCGAGTCGACCGTGTACGTCTCCCCCCGCCACCAGCGGACGGGACTGGGC TCCACGCTCTACACCCACCTGCTGAAGTCCCTGGAGGCACAGGGCTTCAAG AGCGTGGTCGCTGTCATCGGGCTGCCCAACGACCCGAGCGTGCGCATGCA CGAGGCGCTCGGATATGCCCCCCGCGGCATGCTGCGGGCGGCCGGCTTC AAGCACGGGAACTGGCATGACGTGGGTTTCTGGCAGCTGGACTTCAGCCT GCCGGTACCGCCCCGTCCGGTCCTGCCCGTCACCGAGATCTGATCCGTCG ACCTGCAGATCGTTCAAACATTTGGCAATAAAGTTTCTTAAGATTGAATCCTG TTGCCGGTCTTGCGATGATTATCATATAATTTCTGTTGAATTACGTTAAGCAT GTAATAATTAACATGTAATGCATGACGTTATTTATGAGATGGGTTTTTATGAT 
TAGAGTCCCGCAATTATACATTTAATACGCGATAGAAAACAAAATATAGCGC GCAAACTAGGATAAATTATCGCGCGCGGTGTCATCTATGTTACTAGATCCGA TGATAAGCTGTCAAACATGAGAATTCgatTTTGCATGATATCTTTCCCATAACT TCGTATAGCATACATTATACGAAGTTATGGTTTTCCCCCAACTGGGGTAACC TTTGAGTTCTCTCAGTTGGGGGGGCGGCCGCAAAGGCGCGCCAAAAAAAG GCCGGCCGACGTCTTAATTAAGCGATCGCTAGCTTGGCACTGGCCGTCGTT TTACAACGTCGTGACTGGGAAAACCCTGGCGTTACCCAACTTAATCGCCTT GCAGCACATCCCCCTTTCGCCAGCTGGCGTAATAGCGAAGAGGCCCGCAC CGATCGCCCTTCCCAACAGTTGCGCAGCCTGAATGGCGAATGAGCTTGAGC TTGGATCAGATTGTCGTTTCCCGCCTTCAGTTTAAACTATCAGTGTTTGACA GGATATATTGGCGGGTAAACCTAAGAGAAAAGAGCGTTTATTAGAATAACGG ATATTTAAAAGGGCGTGAAAAGGTTTATCCGTTCGTCCATTTGTATGTGCAT GCCAACCACAGGGTTCCCCTCGGGATCAA

\section{AC1}

CTCCCATTCGCCATTCAGGCTGCGCAACTGTTGGGAAGGGCGATCGGTGC GGGCCTCTTCGCTATTACGCCAGCTGGCGAAAGGGGGATGTGCTGCAAGG CGATTAAGTTGGGTAACGCCAGGGTTTTCCCAGTCACGACGTTGTAAAACG ACGGCCAGTGAGCGCGCGTAATACGACTCACTATAGGGCGAATTGGGTAC CGGGCCCCCCCTCGAGGTCGACGGTATCGATAAGCTTGAGAGAATAACTTC GTATAGCATACATTATACGAAGTTATGAGAGAGTGCCAGGGCGTGCCCTTG GGCTCCCCGGGCGCGGAGAGACCTGCAGGTGATGAGAGATCGTTCAAACA TTTGGCAATAAAGTTTCTTAAGATTGAATCCTGTTGCCGGTCTTGCGATGATT ATCATATAATTTCTGTTGAATTACGTTAAGCATGTAATAATTAACATGTAATGC 
ATGACGTTATTTATGAGATGGGTTTTTATGATTAGAGTCCCGCAATTATACAT TTAATACGCGATAGAAAACAAAATATAGCGCGCAAACTAGGATAAATTATCG CGCGCGGTGTCATCTATGTTACTAGATCTTGACGAGACCTGCAGGTCGACT CTAGAGGATCCCCAACCGCGGTCCCTAGGCCGGCCGCTAGCCATTTAAATG CATGCTGCAGCCCGGGCGACGTCGACTGTTTAAACGAGGCCTTAATTAAGG GCCCAATTGGCCACGTACGTCTAGATCTGGCGCGCCTCGAGCGAGAGAGA GCTCGAGAGATTATAAGAGAGAGTGCCAGGGCGTGCCCTTGGGCTCCCCG GGCGCGGAGAGAACTAGTGGATCCATCGATTAGGAAGTAACCATGAGCCCA GAACGACGCCCGGCCGACATCCGCCGTGCCACCGAGGCGGACATGCCGG CGGTCTGCACCATCGTCAACCACTACATCGAGACAAGCACGGTCAACTTCC GTACCGAGCCGCAGGAACCGCAGGAGTGGACGGACGACCTCGTCCGTCTG CGGGAGCGCTATCCCTGGCTCGTCGCCGAGGTGGACGGCGAGGTCGCCG GCATCGCCTACGCGGGCCCCTGGAAGGCACGCAACGCCTACGACTGGACG GCCGAGTCGACCGTGTACGTCTCCCCCCGCCACCAGCGGACGGGACTGG GCTCCACGCTCTACACCCACCTGCTGAAGTCCCTGGAGGCACAGGGCTTCA AGAGCGTGGTCGCTGTCATCGGGCTGCCCAACGACCCGAGCGTGCGCATG CACGAGGCGCTCGGATATGCCCCCCGCGGCATGCTGCGGGCGGCCGGCT TCAAGCACGGGAACTGGCATGACGTGGGTTTCTGGCAGCTGGACTTCAGC CTGCCGGTACCGCCCCGTCCGGTCCTGCCCGTCACCGAGATCTGATCCGT CGACCTGCAGATCGTTCAAACATTTGGCAATAAAGTTTCTTAAGATTGAATC CTGTTGCCGGTCTTGCGATGATTATCATATAATTTCTGTTGAATTACGTTAAG CATGTAATAATTAACATGTAATGCATGACGTTATTTATGAGATGGGTTTTTAT GATTAGAGTCCCGCAATTATACATTTAATACGCGATAGAAAACAAAATATAG 
CGCGCAAACTAGGATAAATTATCGCGCGCGGTGTCATCTATGTTACTAGATC CGATGATAAGCTGTCAAACATGAGAATTCGATTTTGCATGATATCGAATTCC TGCAGCCCGGGGGATCCACTAGTTCTAGAGCGGCCGCCACCGCGGTGGAG CTCCAGCTTTTGTTCCCTTTAGTGAGGGTTAATTGCGCGCTTGGCGTAATCA TGGTCATAGCTGTTTCCTGTGTGAAATTGTTATCCGCTCACAATTCCACACA ACATACGAGCCGGAAGCATAAAGTGTAAAGCCTGGGGTGCCTAATGAGTGA GCTAACTCACATTAATTGCGTTGCGCTCACTGCCCGCTTTCCAGTCGGGAAA CCTGTCGTGCCAGCTGCATTAATGAATCGGCCAACGCGCGGGGAGAGGCG GTTTGCGTATTGGGCGCTCTTCCGCTTCCTCGCTCACTGACTCGCTGCGCT CGGTCGTTCGGCTGCGGCGAGCGGTATCAGCTCACTCAAAGGCGGTAATA CGGTTATCCACAGAATCAGGGGATAACGCAGGAAAGAACATGTGAGCAAAA GGCCAGCAAAAGGCCAGGAACCGTAAAAAGGCCGCGTTGCTGGCGTTTTT CCATAGGCTCCGCCCCCCTGACGAGCATCACAAAAATCGACGCTCAAGTCA GAGGTGGCGAAACCCGACAGGACTATAAAGATACCAGGCGTTTCCCCCTG GAAGCTCCCTCGTGCGCTCTCCTGTTCCGACCCTGCCGCTTACCGGATACC TGTCCGCCTTTCTCCCTTCGGGAAGCGTGGCGCTTTCTCATAGCTCACGCT GTAGGTATCTCAGTTCGGTGTAGGTCGTTCGCTCCAAGCTGGGCTGTGTGC ACGAACCCCCCGTTCAGCCCGACCGCTGCGCCTTATCCGGTAACTATCGTC TTGAGTCCAACCCGGTAAGACACGACTTATCGCCACTGGCAGCAGCCACTG GTAACAGGATTAGCAGAGCGAGGTATGTAGGCGGTGCTACAGAGTTCTTGA AGTGGTGGCCTAACTACGGCTACACTAGAAGGACAGTATTTGGTATCTGCG CTCTGCTGAAGCCAGTTACCTTCGGAAAAAGAGTTGGTAGCTCTTGATCCG GCAAACAAACCACCGCTGGTAGCGGTGGTTTTTTTGTTTGCAAGCAGCAGA 
TTACGCGCAGAAAAAAAGGATCTCAAGAAGATCCTTTGATCTTTTCTACGGG GTCTGACGCTCAGTGGAACGAAAACTCACGTTAAGGGATTTTGGTCATGAG ATTATCAAAAAGGATCTTCACCTAGATCCTTTTAAATTAAAAATGAAGTTTTAA ATCAATCTAAAGTATATATGAGTAAACTTGGTCTGACAGTTACCAATGCTTAA TCAGTGAGGCACCTATCTCAGCGATCTGTCTATTTCGTTCATCCATAGTTGC CTGACTCCCCGTCGTGTAGATAACTACGATACGGGAGGGCTTACCATCTGG CCCCAGTGCTGCAATGATACCGCGAGACCCACGCTCACCGGCTCCAGATTT ATCAGCAATAAACCAGCCAGCCGGAAGGGCCGAGCGCAGAAGTGGTCCTG CAACTTTATCCGCCTCCATCCAGTCTATTAATTGTTGCCGGGAAGCTAGAGT AAGTAGTTCGCCAGTTAATAGTTTGCGCAACGTTGTTGCCATTGCTACAGGC ATCGTGGTGTCACGCTCGTCGTTTGGTATGGCTTCATTCAGCTCCGGTTCC CAACGATCAAGGCGAGTTACATGATCCCCCATGTTGTGCAAAAAAGCGGTT AGCTCCTTCGGTCCTCCGATCGTTGTCAGAAGTAAGTTGGCCGCAGTGTTA TCACTCATGGTTATGGCAGCACTGCATAATTCTCTTACTGTCATGCCATCCG TAAGATGCTTTTCTGTGACTGGTGAGTACTCAACCAAGTCATTCTGAGAATA GTGTATGCGGCGACCGAGTTGCTCTTGCCCGGCGTCAATACGGGATAATAC CGCGCCACATAGCAGAACTTTAAAAGTGCTCATCATTGGAAAACGTTCTTCG GGGCGAAAACTCTCAAGGATCTTACCGCTGTTGAGATCCAGTTCGATGTAA CCCACTCGTGCACCCAACTGATCTTCAGCATCTTTTACTTTCACCAGCGTTT CTGGGTGAGCAAAAACAGGAAGGCAAAATGCCGCAAAAAAGGGAATAAGG GCGACACGGAAATGTTGAATACTCATACTCTTCCTTTTTCAATATTATTGAAG CATTTATCAGGGTTATTGTCTCATGAGCGGATACATATTTGAATGTATTTAGA AAAATAAACAAATAGGGGTTCCGCGCACATTTCCCCGAAAAGTGCCAC 


\section{AC2}

CTCCCATTCGCCATTCAGGCTGCGCAACTGTTGGGAAGGGCGATCGGTGC GGGCCTCTTCGCTATTACGCCAGCTGGCGAAAGGGGGATGTGCTGCAAGG CGATTAAGTTGGGTAACGCCAGGGTTTTCCCAGTCACGACGTTGTAAAACG ACGGCCAGTGAGCGCGCGTAATACGACTCACTATAGGGCGAATTGGGTAC CGGGCCCCCCCTCGAGGTCGACGGTATCGATAAGCTTGAGAGAATAACTTC GTATAGCATACATTATACGAAGTTATGAGAGACCCCAACTGGGGTAACCTTT GAGTTCTCTCAGTTGGGGGGAGAGACCTGCAGGTGATGAGAGATCGTTCAA ACATTTGGCAATAAAGTTTCTTAAGATTGAATCCTGTTGCCGGTCTTGCGAT GATTATCATATAATTTCTGTTGAATTACGTTAAGCATGTAATAATTAACATGTA ATGCATGACGTTATTTATGAGATGGGTTTTTATGATTAGAGTCCCGCAATTAT ACATTTAATACGCGATAGAAAACAAAATATAGCGCGCAAACTAGGATAAATT ATCGCGCGCGGTGTCATCTATGTTACTAGATCTTGACGAGACCTGCAGGTC GACTCTAGAGGATCCCCAACCGCGGTCCCTAGGCCGGCCGCTAGCCATTT AAATGCATGCTGCAGCCCGGGCGACGTCGACTGTTTAAACGAGGCCTTAAT TAAGGGCCCAATTGGCCACGTACGTCTAGATCTGGCGCGCCTCGAGCGAG AGAGAGCTCGAGAGATTATAAGAGAGACCCCAACTGGGGTAACCTTTGAGT TCTCTCAGTTGGGGGGAGAGAACTAGTGGATCCATCGATTAGGAAGTAACC ATGAGCCCAGAACGACGCCCGGCCGACATCCGCCGTGCCACCGAGGCGG ACATGCCGGCGGTCTGCACCATCGTCAACCACTACATCGAGACAAGCACGG TCAACTTCCGTACCGAGCCGCAGGAACCGCAGGAGTGGACGGACGACCTC GTCCGTCTGCGGGAGCGCTATCCCTGGCTCGTCGCCGAGGTGGACGGCGA GGTCGCCGGCATCGCCTACGCGGGCCCCTGGAAGGCACGCAACGCCTAC 
GACTGGACGGCCGAGTCGACCGTGTACGTCTCCCCCCGCCACCAGCGGAC GGGACTGGGCTCCACGCTCTACACCCACCTGCTGAAGTCCCTGGAGGCAC AGGGCTTCAAGAGCGTGGTCGCTGTCATCGGGCTGCCCAACGACCCGAGC GTGCGCATGCACGAGGCGCTCGGATATGCCCCCCGCGGCATGCTGCGGG CGGCCGGCTTCAAGCACGGGAACTGGCATGACGTGGGTTTCTGGCAGCTG GACTTCAGCCTGCCGGTACCGCCCCGTCCGGTCCTGCCCGTCACCGAGAT CTGATCCGTCGACCTGCAGATCGTTCAAACATTTGGCAATAAAGTTTCTTAA GATTGAATCCTGTTGCCGGTCTTGCGATGATTATCATATAATTTCTGTTGAAT TACGTTAAGCATGTAATAATTAACATGTAATGCATGACGTTATTTATGAGATG GGTTTTTATGATTAGAGTCCCGCAATTATACATTTAATACGCGATAGAAAACA AAATATAGCGCGCAAACTAGGATAAATTATCGCGCGCGGTGTCATCTATGTT ACTAGATCCGATGATAAGCTGTCAAACATGAGAATTCGATTTTGCATGATAT CGAATTCCTGCAGCCCGGGGGATCCACTAGTTCTAGAGCGGCCGCCACCG CGGTGGAGCTCCAGCTTTTGTTCCCTTTAGTGAGGGTTAATTGCGCGCTTG GCGTAATCATGGTCATAGCTGTTTCCTGTGTGAAATTGTTATCCGCTCACAA TTCCACACAACATACGAGCCGGAAGCATAAAGTGTAAAGCCTGGGGTGCCT AATGAGTGAGCTAACTCACATTAATTGCGTTGCGCTCACTGCCCGCTTTCCA GTCGGGAAACCTGTCGTGCCAGCTGCATTAATGAATCGGCCAACGCGCGG GGAGAGGCGGTTTGCGTATTGGGCGCTCTTCCGCTTCCTCGCTCACTGACT CGCTGCGCTCGGTCGTTCGGCTGCGGCGAGCGGTATCAGCTCACTCAAAG GCGGTAATACGGTTATCCACAGAATCAGGGGATAACGCAGGAAAGAACATG TGAGCAAAAGGCCAGCAAAAGGCCAGGAACCGTAAAAAGGCCGCGTTGCT GGCGTTTTTCCATAGGCTCCGCCCCCCTGACGAGCATCACAAAAATCGACG 
CTCAAGTCAGAGGTGGCGAAACCCGACAGGACTATAAAGATACCAGGCGTT TCCCCCTGGAAGCTCCCTCGTGCGCTCTCCTGTTCCGACCCTGCCGCTTAC CGGATACCTGTCCGCCTTTCTCCCTTCGGGAAGCGTGGCGCTTTCTCATAG CTCACGCTGTAGGTATCTCAGTTCGGTGTAGGTCGTTCGCTCCAAGCTGGG CTGTGTGCACGAACCCCCCGTTCAGCCCGACCGCTGCGCCTTATCCGGTAA CTATCGTCTTGAGTCCAACCCGGTAAGACACGACTTATCGCCACTGGCAGC AGCCACTGGTAACAGGATTAGCAGAGCGAGGTATGTAGGCGGTGCTACAG AGTTCTTGAAGTGGTGGCCTAACTACGGCTACACTAGAAGGACAGTATTTG GTATCTGCGCTCTGCTGAAGCCAGTTACCTTCGGAAAAAGAGTTGGTAGCT CTTGATCCGGCAAACAAACCACCGCTGGTAGCGGTGGTTTTTTTGTTTGCAA GCAGCAGATTACGCGCAGAAAAAAAGGATCTCAAGAAGATCCTTTGATCTTT TCTACGGGGTCTGACGCTCAGTGGAACGAAAACTCACGTTAAGGGATTTTG GTCATGAGATTATCAAAAAGGATCTTCACCTAGATCCTTTTAAATTAAAAATG AAGTTTTAAATCAATCTAAAGTATATATGAGTAAACTTGGTCTGACAGTTACC AATGCTTAATCAGTGAGGCACCTATCTCAGCGATCTGTCTATTTCGTTCATC CATAGTTGCCTGACTCCCCGTCGTGTAGATAACTACGATACGGGAGGGCTT ACCATCTGGCCCCAGTGCTGCAATGATACCGCGAGACCCACGCTCACCGG CTCCAGATTTATCAGCAATAAACCAGCCAGCCGGAAGGGCCGAGCGCAGAA GTGGTCCTGCAACTTTATCCGCCTCCATCCAGTCTATTAATTGTTGCCGGGA AGCTAGAGTAAGTAGTTCGCCAGTTAATAGTTTGCGCAACGTTGTTGCCATT GCTACAGGCATCGTGGTGTCACGCTCGTCGTTTGGTATGGCTTCATTCAGC TCCGGTTCCCAACGATCAAGGCGAGTTACATGATCCCCCATGTTGTGCAAA AAAGCGGTTAGCTCCTTCGGTCCTCCGATCGTTGTCAGAAGTAAGTTGGCC 
GCAGTGTTATCACTCATGGTTATGGCAGCACTGCATAATTCTCTTACTGTCA TGCCATCCGTAAGATGCTTTTCTGTGACTGGTGAGTACTCAACCAAGTCATT CTGAGAATAGTGTATGCGGCGACCGAGTTGCTCTTGCCCGGCGTCAATACG GGATAATACCGCGCCACATAGCAGAACTTTAAAAGTGCTCATCATTGGAAAA CGTTCTTCGGGGCGAAAACTCTCAAGGATCTTACCGCTGTTGAGATCCAGTT CGATGTAACCCACTCGTGCACCCAACTGATCTTCAGCATCTTTTACTTTCAC CAGCGTTTCTGGGTGAGCAAAAACAGGAAGGCAAAATGCCGCAAAAAAGGG AATAAGGGCGACACGGAAATGTTGAATACTCATACTCTTCCTTTTTCAATATT ATTGAAGCATTTATCAGGGTTATTGTCTCATGAGCGGATACATATTTGAATGT ATTTAGAAAAATAAACAAATAGGGGTTCCGCGCACATTTCCCCGAAAAGTGC CAC 


\section{VITA}

Nathan Charles Swyers was born December 31, 1988 in Jefferson City, Missouri to his parent, Brenda Sue Swyers and Charles Randolph Swyers. Nathan graduated high school in 2007 from Maries R-1 High School, located in Vienna, Missouri. He attended the University of Missouri where he graduated with a BS of Biology in 2011. Nathan started his graduate work at the University of Missouri in the lab of Dr. James Birchler in the Fall of 2012. Nathan was married to Amelia Ruth Noble on August 30, 2014 in St. Charles, Missouri. Gideon Michael Swyers was born to Nathan and Amelia on November 17, 2016 in Jefferson City, Missouri. 